Instituto Universitario de Oftalmobiología Aplicada - IOBA

Doctorado en Ciencias de la Visión

TESIS DOCTORAL:

\title{
EFECTOS DE LA POSTURA CORPORAL, EQUILIBRIO Y DESTREZA MANUAL EN LA VELOCIDAD LECTORA EN PERSONAS MAYORES CON Y SIN BAJA VISIÓN
}

Presentada por Ana Patrícia de Queiroz Barbosa para optar al grado de doctora por la Universidad de Valladolid

Dirigida por: Dra. Valle Flores Lucas

Dra. Begoña Coco Martín

Dra. Teresa del Álamo Martín 

A mi familia 

"Todas as estradas são floridas, mesmo que os nossos olhos as vejam em forma de espinhos. Na profundidade, são flores que educam e instruem. É por isso que chamamos o ingresso na carne de chão de rosas".

Irmã Scheilla

"Alégrate cuando llegues a la cumbre: llora, bate palmas, grita a los cuatro vientos que lo has conseguido, deja que el viento allá en lo alto (porque allá en la cima siempre hace viento) purifique tu mente, refresca tus pies sudados y cansados, abre los ojos, limpia el polvo de tu corazón. Piensa que lo que antes era apenas un sueño, una visión lejana, es ahora parte de tu vida. Lo conseguiste".

Paulo Coelho 

ÍNDICE 



\section{Índice}

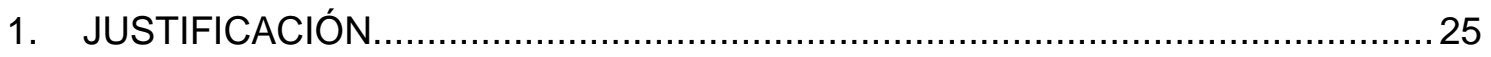

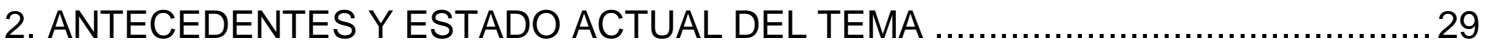

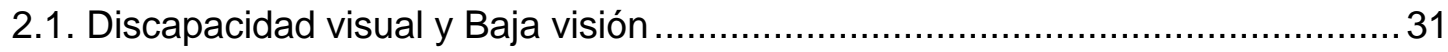

2.2. La Columna Vertebral: Anatomía y Biomecánica............................................... 34

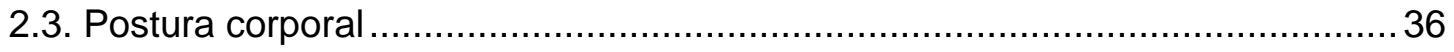

2.3.1. Postura Corporal y Baja Visión ............................................................. 38

2.3.2. Métodos de Evaluación Postural................................................................. 41

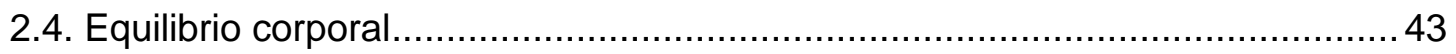

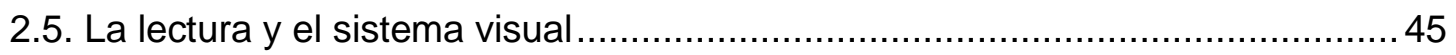

2.5.1. La velocidad lectora y la discapacidad visual ..........................................4 47

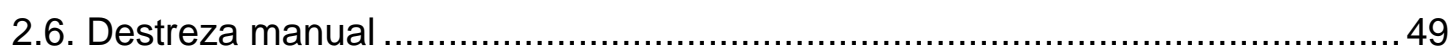

2.7. Repercusiones psicológicas de la Discapacidad Visual ....................................51

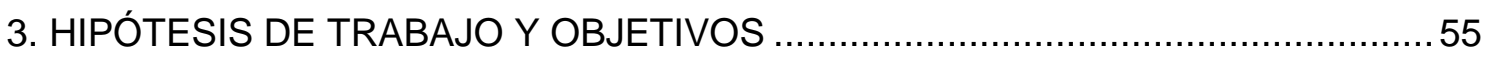

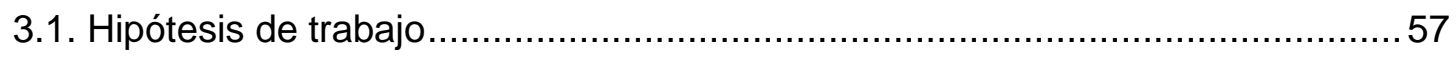

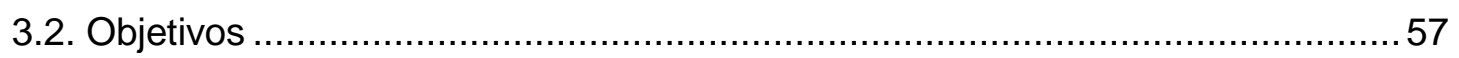

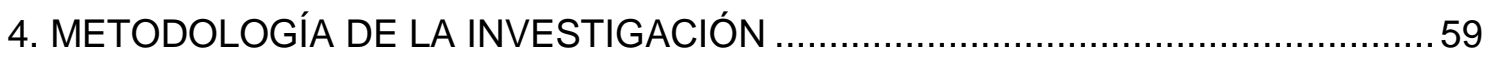

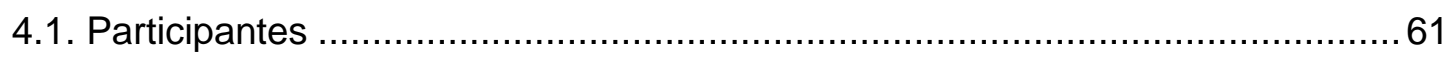

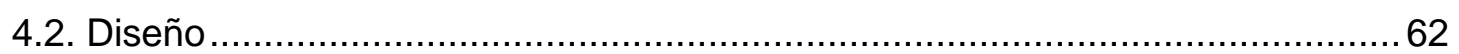

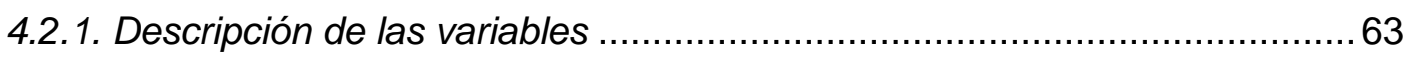

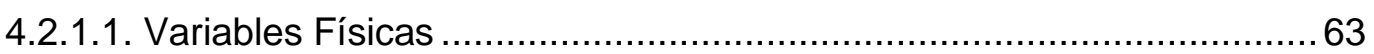

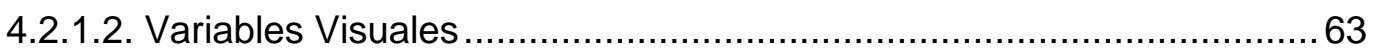

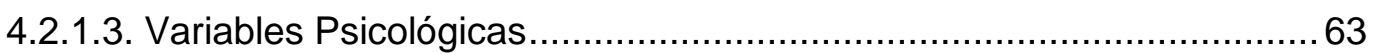

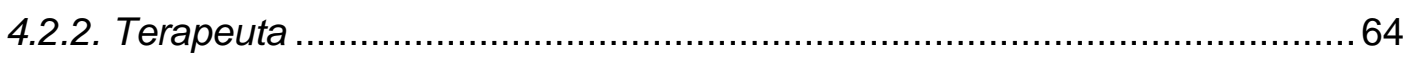

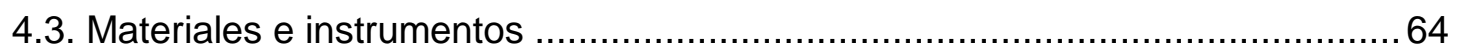

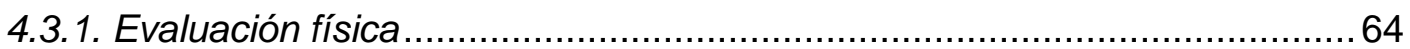

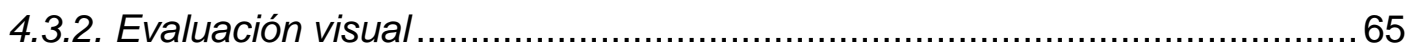

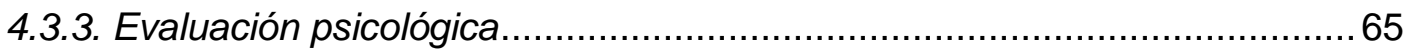

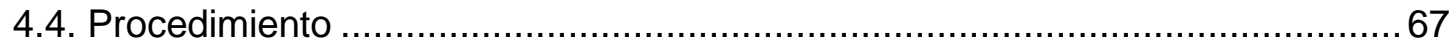

4.4.1. Captación de los participantes ...........................................................

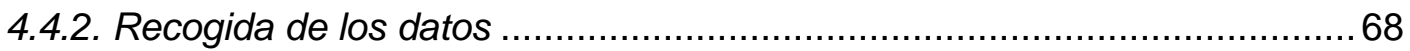

4.4.3. Preparación y posicionamiento de los participantes....................................69

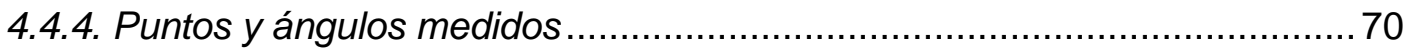

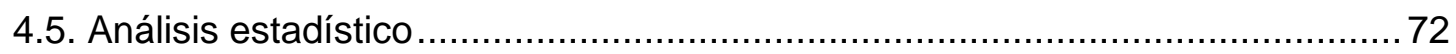




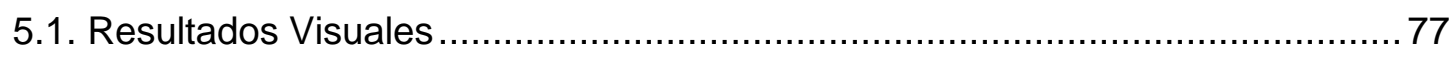

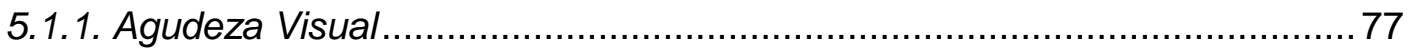

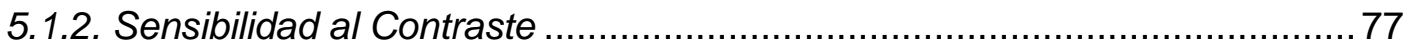

5.1.3. Ayudas Ópticas y Patologías visuales.................................................. 78

5.2. Fiabilidad y validez del sensor postural LUMOback..................................... 79

5.3. Fiabilidad intraobservador de las medidas posturales utilizando el protocolo

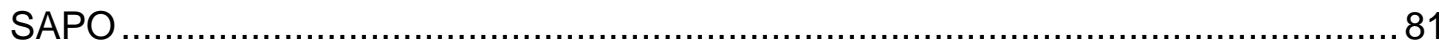

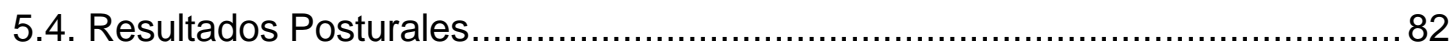

5.4.1. Evaluación postural en sedestación .......................................................... 82

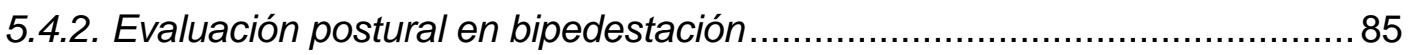

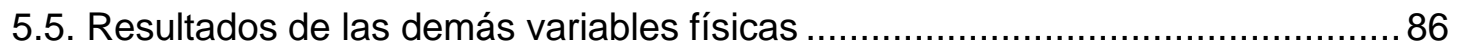

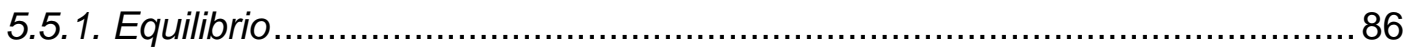

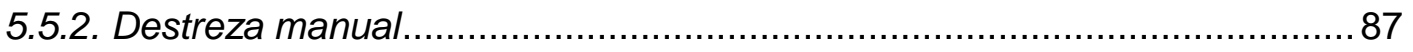

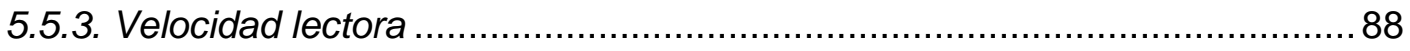

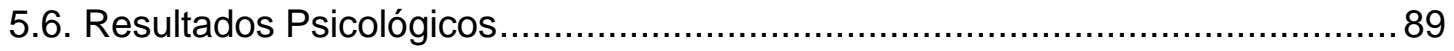

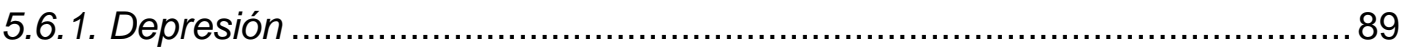

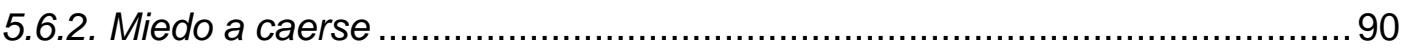

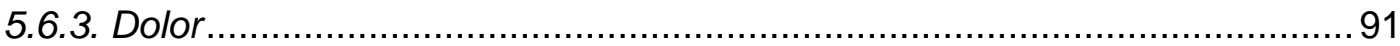

5.7. Resultados de la correlación entre las variables ............................................. 93

5.7.1. Relación entre la VL y la Postura Corporal................................................ 93

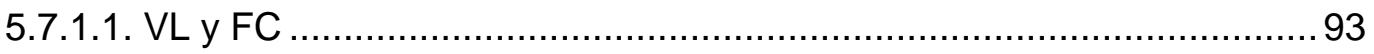

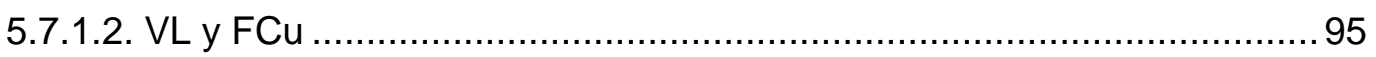

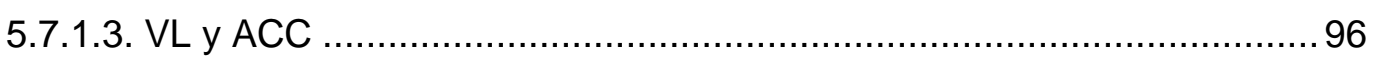

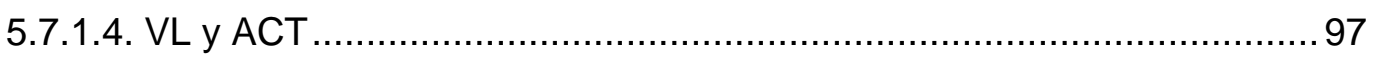

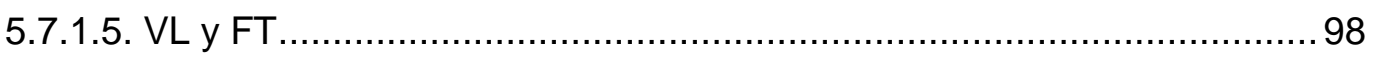

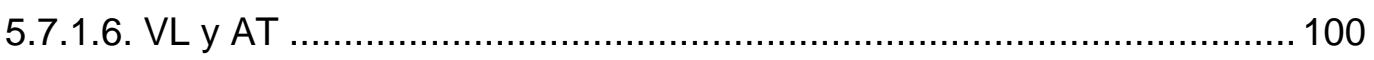

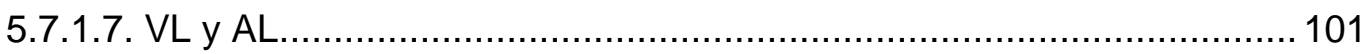

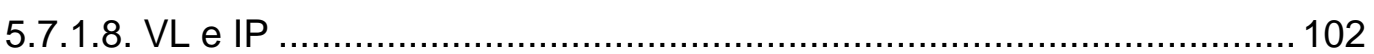

5.7.2. Relación entre la VL y el Equilibrio Corporal ........................................... 103

5.7.3. Relación entre la VL y la Destreza Manual.............................................. 104

5.7.4. Relación entre la VL y la Función Visual.................................................. 106

5.7.5. Relación entre la VL y la Depresión .......................................................... 107

5.7.6. Relación entre la Depresión y la Postura Corporal................................... 108

5.7.7. Relación entre la Depresión y el Equilibrio Corporal ................................ 111 
5.7.9. Relación entre la Depresión y el Miedo a Caerse....................................... 114

5.7.10. Relación entre la Depresión y el Dolor ................................................. 115

5.7.11. Análisis multivariante ...................................................................... 117

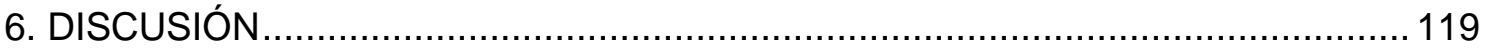

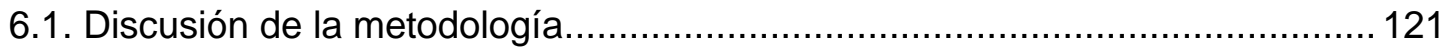

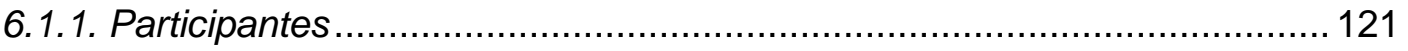

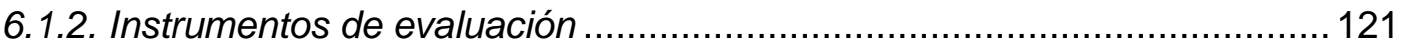

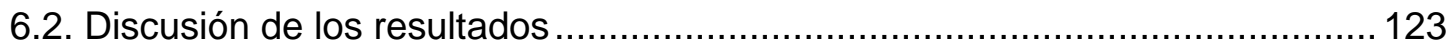

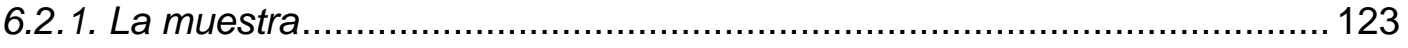

6.2.2. Fiabilidad y validez del sensor postural.............................................. 124

6.2.3. Fiabilidad en la demarcación de los puntos anatómicos........................... 126

6.2.4. Discusión de los resultados de la Postura en sedestación: comparación

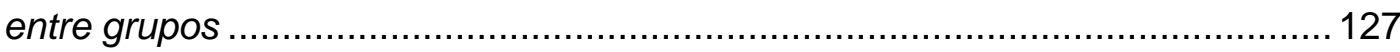

6.2.5. Discusión de los resultados de la Postura en bipedestación: comparación

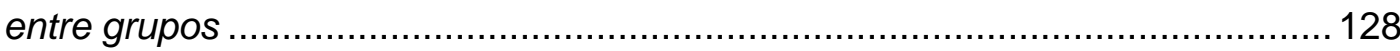

6.2.6. Discusión de las variables físicas equilibrio, destreza manual y velocidad lectora: comparación entre ambos grupos ......................................................... 130

6.2.7. Discusión de los resultados de las variables visuales: comparación entre grupos

6.2.8. Discusión de los resultados de las variables psicológicas: comparación entre grupos

6.2.8.1. Sintomatología depresiva en ambos grupos ................................... 133

6.2.8.2. El miedo a caerse en ambos grupos ............................................ 134

6.2.8.3. El dolor percibido en ambos grupos .............................................. 135

6.2.9. Discusión de los resultados de la correlación entre las variables .............. 136

6.2.9.1. La VL y la Postura corporal en sedestación...................................... 136

6.2.9.2. La VL y el Equilibrio corporal .................................................... 137

6.2.9.3. La VL y la Destreza manual......................................................... 139

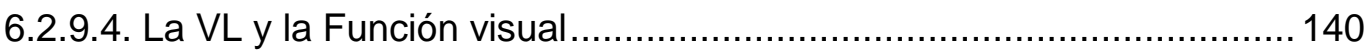

6.2.9.5. La VL y la Depresión .................................................................. 140

6.2.9.6. La Depresión y la Postura corporal.................................................... 141

6.2.9.7. La Depresión y la Equilibrio corporal ............................................ 142

6.2.9.8. La Depresión y la Destreza manual ................................................. 142

6.2.9.9. La Depresión y el Miedo a caerse .................................................... 143

6.2.9.10. La Depresión y el Dolor .................................................................. 144 


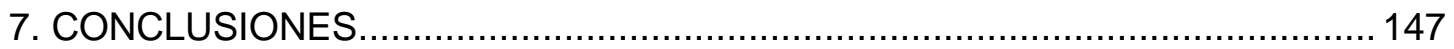

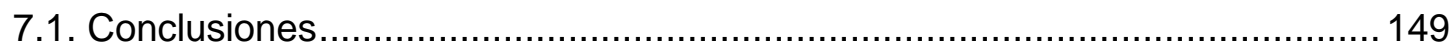

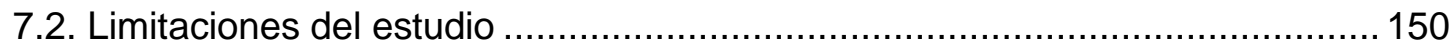

7.3. Continuación de esta línea de investigación ................................................ 150

7.4. Implicaciones para la práctica clínica ....................................................... 150

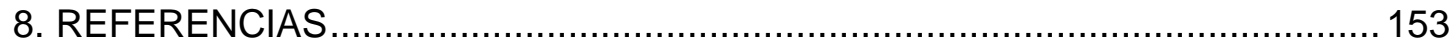

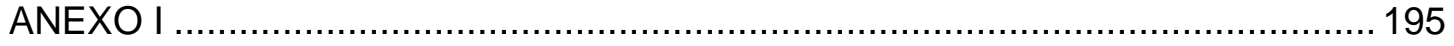

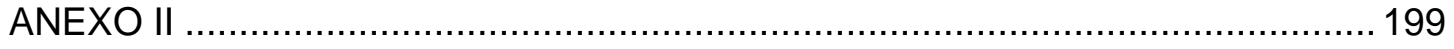

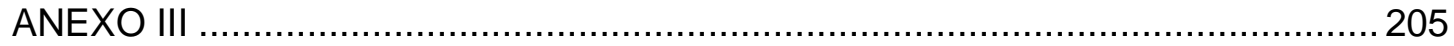

\section{Índice de Figuras}

Figura 1: A. Disminución de la SC. B. Pérdida de CV periférico. C. Pérdida de CV

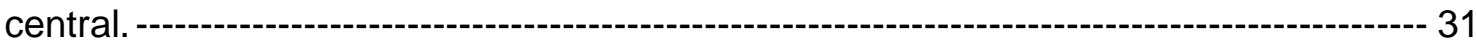

Figura 2: Unidad funcional normal de la columna vertebral. ---------------------------- 34

Figura 3: Cambios en las curvaturas vertebrales. A. Curvatura convexa posterior. B. Desarrollo de la lordosis cervical. C. Alrededor de los 10 años de edad, todas las curvaturas fisiológicas son iguales a las de un adulto. D. Curvaturas de la columna vertebral. ----------------------------------------------------------------------------- 35

Figura 4: Curvaturas anormales de la columna. ---

Figura 5: Músculos agonista y antagonista para la flexión del brazo. ------------- 37

Figura 6: Fuerzas de compresión y tensión en el disco intervertebral.------------- 37

Figura 7: A. Columna torácica en vertical. B. Columna inclinada. C. Columna lumbar en

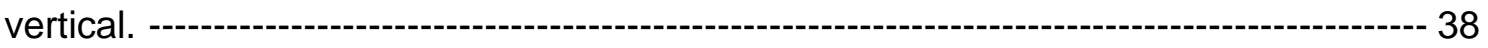

Figura 8: Reflejo vestíbulo-ocular lineal.-------------- 39

Figura 9: Relación entre la posición de la cabeza y la columna cervical.------------- 40

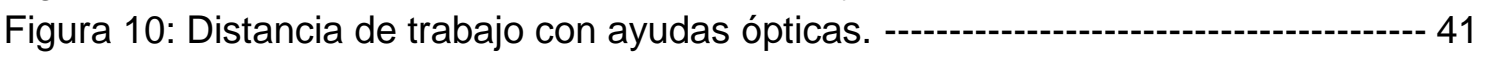

Figura 11: Movimientos oculares precisos a lo largo de la línea y retorno al principio de

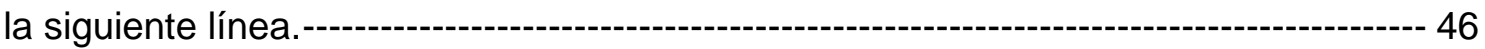

Figura 12: Tipos de lupas. ------.-- 48

Figura 13: LUMOback------ 65

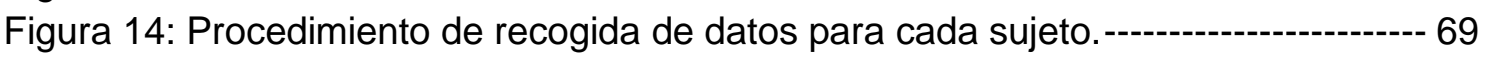

Figura 15: Fases del análisis de datos. ----------- 72

Figura 16: FC de los grupos caso (en rojo) y control (en negro).------------------- 83

Figura 17: FCu de los grupos caso (en rojo) y control (en negro). -

Figura 18: FT de los grupos caso (en rojo) y control (en negro). -------------------- 84

Figura 19: AT de los grupos caso (en rojo) y control (en negro). ------------------ 84

Figura 20: IP de los grupos caso (en rojo) y control (en negro). --- 


\section{Índice de Gráficos}

Gráfico 1: Distribución de la utilización de ayudas ópticas para lectura.----------------- 79

Gráfico 2: Distribución por patología visual en el grupo caso (\%). ------------ 79

Gráfico 3: Acuerdo de medición entre los ángulos lumbares registrados con el SAPO y

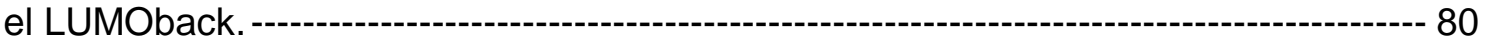

Gráfico 4: Correlación entre los valores de los ángulos lumbares medidos por el LUMOback y el SAPO en ambos grupos. ---

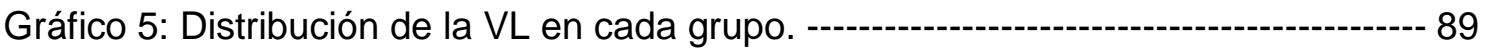

\section{Índice de Tablas}

Tabla 1: Clasificación de la gravedad de la discapacidad visual.--------------------------- 32

Tabla 2: Criterios de inclusión de los participantes.---------------------------------------- 61

Tabla 3: Criterios de exclusión de los participantes.

Tabla 4: Variables evaluadas. --.-- 62

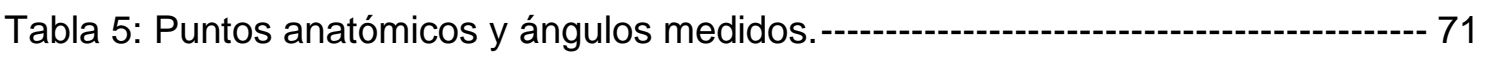

Tabla 6: Datos descriptivos y contrastes de normalidad y homogeneidad de varianzas para la AV en ambos grupos. ------------------------------------------ 77

Tabla 7: Contraste de igualdad de medianas para la AV en ambos grupos. ------------ 77 Tabla 8: Datos descriptivos y contrastes de normalidad y homogeneidad de varianzas para la SC en ambos grupos. ----------------------------------------------------- 78

Tabla 9: Contraste de igualdad de medianas para la SC en ambos grupos. ------------ 78

Tabla 10: Resultados de la repetibilidad intrasesión. ------- 80

Tabla 11: Datos estadísticos del acuerdo entre días de las medidas posturales. ------ 81

Tabla 12: Datos descriptivos y contrastes de normalidad y homogeneidad de varianzas para los ángulos posturales en sedestación en ambos grupos. -------------------- 82

Tabla 13: Contraste de igualdad de medias para los ángulos posturales en

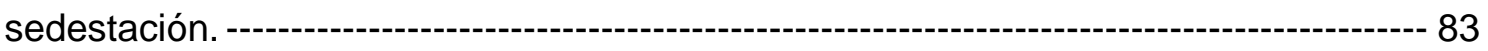

Tabla 14: Datos descriptivos y contrastes de normalidad y homogeneidad de varianzas para los ángulos posturales en bipedestación en ambos grupos. ----------------------- 85 Tabla 15: Contraste de igualdad de medias para los ángulos posturales en

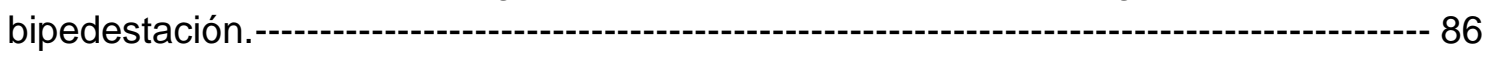

Tabla 16: Datos descriptivos y contrastes de normalidad y homogeneidad de varianzas

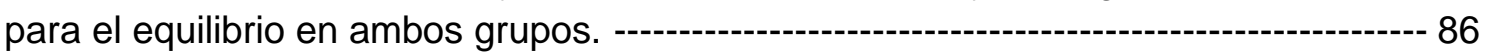

Tabla 17: Contraste de igualdad de medianas para el equilibrio. ------------------------ 87

Tabla 18: Datos descriptivos y contrastes de normalidad y homogeneidad de varianzas para la destreza manual en ambos grupos. -------------------------- 87

Tabla 19: Contraste de igualdad de medianas en la evaluación de la destreza manual.

Tabla 20: Datos descriptivos y contrastes de normalidad y homogeneidad de varianzas para la VL en ambos grupos.--------------------------------------------- 89

Tabla 21: Contraste de igualdad de medianas en la evaluación de la VL. -------------- 89 Tabla 22: Datos descriptivos y contrastes de normalidad y homogeneidad de varianzas para la sintomatología depresiva en ambos grupos. 
Tabla 23: Contraste de igualdad de medianas para la sintomatología depresiva en

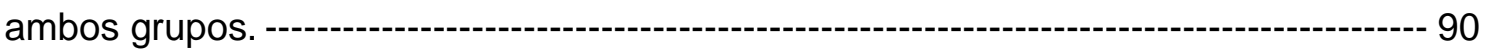

Tabla 24: Datos descriptivos y contrastes de normalidad y homogeneidad de varianzas para el miedo a caerse en ambos grupos.

Tabla 25: Contraste de igualdad de medianas para el miedo a caerse en ambos

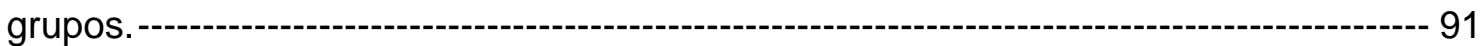

Tabla 26: Datos descriptivos y contrastes de normalidad y homogeneidad de varianzas para el dolor en ambos grupos.---.---

Tabla 27: Contraste de igualdad de medianas para el dolor en ambos grupos. ------- 93

Tabla 28: Correlación entre la VL y la FC. ------------ 93

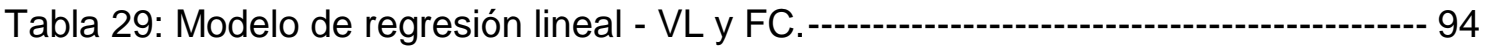

Tabla 30: Bondad de ajuste.-- 94

Tabla 31: Correlación entre la VL y la FCu.--- 95

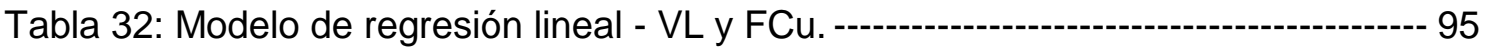

Tabla 33: Bondad de ajuste.-- 96

Tabla 34: Correlación entre la VL y el ACC. -- 96

Tabla 35: Modelo de regresión lineal - VL y ACC. --------------------------------- 97

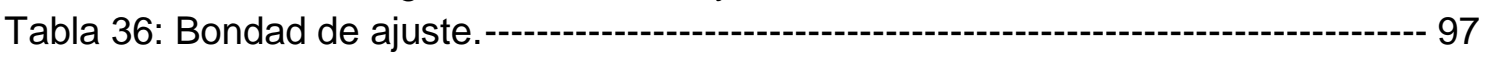

Tabla 37: Correlación entre la VL y el ACT. --------------------------------- 97

Tabla 38: Modelo de regresión lineal - VL y ACT.---.----- 98

Tabla 39: Bondad de ajuste.-- 98

Tabla 40: Correlación entre la VL y la FT.-- 99

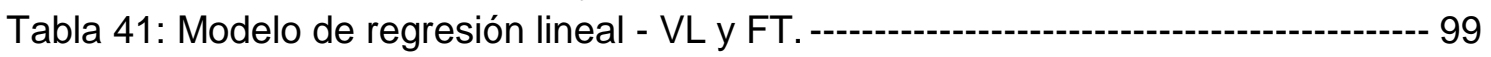

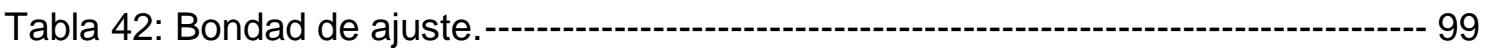

Tabla 43: Correlación entre la VL y el AT. --

Tabla 44: Modelo de regresión lineal - VL y AT. -

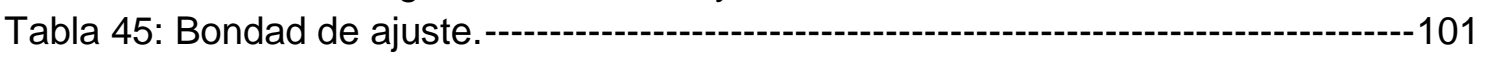

Tabla 46: Correlación entre la VL y el AL.---.--.--101

Tabla 47: Modelo de regresión lineal - VL y AL. ---

Tabla 48: Bondad de ajuste.--

Tabla 49: Correlación entre la VL y la IP. -

Tabla 50: Modelo de regresión lineal - VL y IP. ---

Tabla 51: Bondad de ajuste.------

Tabla 52: Correlación entre la VL y el equilibrio. --

Tabla 53: Modelo de regresión lineal - VL y equilibrio.----

Tabla 54: Bondad de ajuste.---

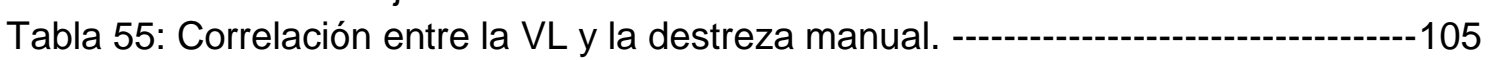

Tabla 56: Modelo de regresión lineal - VL y destreza manual. --------------------------105

Tabla 57: Bondad de ajuste.---------106

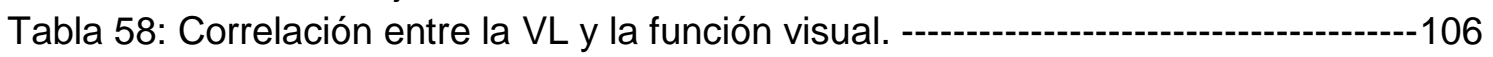

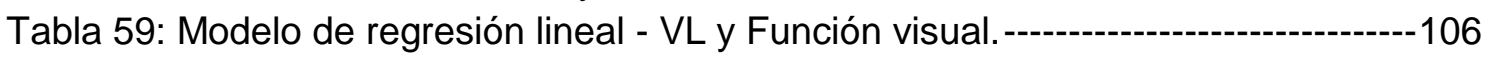

Tabla 60: Bondad de ajuste.--

Tabla 61: Correlación entre la VL y la sintomatología depresiva. -------------------107

Tabla 62: Modelo de regresión lineal - VL y GDS. - -

Tabla 63: Bondad de ajuste. -

Tabla 64: Datos descriptivos y contrastes de normalidad y homogeneidad de varianzas para la relación entre la depresión y la postura en sedestación. -----------------------109 
Tabla 65: Igualdad de medias para la relación entre la depresión y la postura en

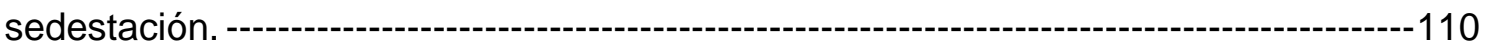

Tabla 66: Modelo de regresión logística - Depresión y Postura en sedestación. ------111

Tabla 67: Datos descriptivos y contrastes de normalidad y homogeneidad de varianzas para la relación entre la depresión y el equilibrio. -------112

Tabla 68: Igualdad de medianas para la relación entre la depresión y el equilibrio. --112

Tabla 69: Modelo de regresión logística - Depresión y Equilibrio.----

Tabla 70: Datos descriptivos y contrastes de normalidad y homogeneidad de varianzas para la relación entre la depresión y la destreza manual. ------o---113

Tabla 71: Igualdad de medianas para la relación entre la depresión y la destreza

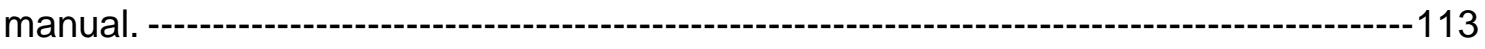

Tabla 72: Modelo de regresión logística - Depresión y Destreza manual. -------------114 Tabla 73: Datos descriptivos y contrastes de normalidad y homogeneidad de varianzas para la relación entre la depresión y el miedo a caerse.-----------------------------114 Tabla 74: Igualdad de medianas para la relación entre la depresión y el miedo a

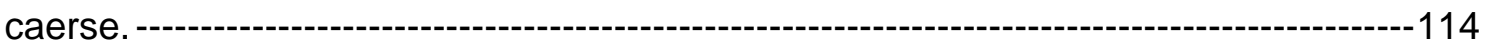

Tabla 75: Modelo de regresión logística - Depresión y Miedo a caerse.-----------------115

Tabla 76: Datos descriptivos y el contraste de independencia para la relación entre la Depresión y el Dolor. ------------------------------------------------------------------115

Tabla 77: Modelo de regresión logística - Depresión y Dolor.-----------------------116

Tabla 78: Correlación entre las variables de interés. -

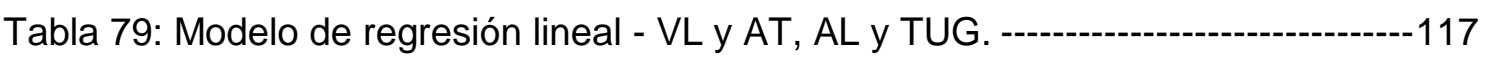

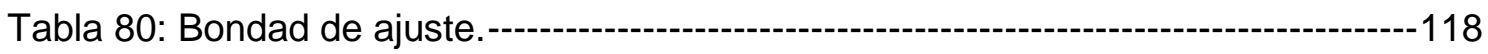





\section{RESUMEN}

Introducción: La pérdida de visión asociada al envejecimiento en las personas mayores supone alteraciones en la postura corporal, equilibrio y destreza manual que interfieren negativamente a la hora de realizar tareas como la lectura. La presente tesis tiene como objetivo evaluar si existen diferencias en cuanto a la aparición y/o severidad de las alteraciones posturales, de equilibrio y destreza manual a mayores con y sin BV. Además, si las alteraciones en la postura y equilibrio corporal y en la destreza manual existentes en las personas mayores con baja visión, interfieren de manera negativa en la velocidad lectora. Conjuntamente, también creemos importante determinar si tales alteraciones tienen algún efecto sobre el estado emocional del individuo.

Métodos: Se evaluó la postura corporal en bipedestación y sedestación, el equilibrio, la destreza manual y la velocidad lectora, además de la sintomatología depresiva, miedo a caerse y dolor percibido en 80 personas mayores con y sin BV. Para ello, se utilizó un diseño cuasiexperimental de caso-control. Se comprobó la fiabilidad de los métodos de medición de la postura utilizados en este estudio y también la correlación entre la velocidad lectora y las variables mencionadas.

Resultados: Para cada ángulo postural medido entre los dos grupos se encontró valores significativamente mayores en el grupo caso para la flexión de cabeza, de cuello, de tórax y la inclinación pélvica, en contrapartida el ángulo del tronco fue significativamente menor en el grupo caso. En lo que se refiere al equilibrio, no hubo diferencias significativas entre ambos grupos, sin embargo la destreza manual en el grupo caso fue peor que en el control. La relación entre la velocidad lectora y 5 ángulos posturales, así como el equilibrio fue estadísticamente significativa $(p<0.05)$ para el grupo control. Se encontró una relación entre la velocidad lectora y la destreza manual en ambos grupos, sin embargo no fue significativa estadísticamente. No se encontró diferencias en ambos grupos para la sintomatología depresiva ni para el dolor, mientras que el miedo a caerse fue mayor en el grupo caso. En lo que se refiere a la fiabilidad del método de evaluación postural no hubo diferencias estadísticamente significativas entre las dos mediciones en ninguno de los ángulos medidos ( $>0.05)$, con una excelente repetibilidad intersesión ( $\mathrm{CCl}>0.95)$.

Conclusiones: Las personas mayores con baja visión tienen la postura corporal más alterada tanto en bipedestación como en sedestación en relación a las personas mayores sin baja visión, del mismo modo su destreza manual es menos eficaz y tienen una mayor preocupación por caerse. La postura y el equilibrio corporal presentan un efecto estadísticamente significativo sobre la velocidad lectora, principalmente en los mayores sin baja visión. Los métodos utilizados para la evaluación postural resultaron ser fiables y válidos. 


En 2011 decidí venir a España con el objetivo único de adquirir conocimientos científicos para mí desarrollo profesional. Lo que no me imaginaba es que esta experiencia iba a ser tan increíble y que iba a conocer a tantas personas importantes que contribuiría inmensamente para mi avance profesional y personal. $Y$ ahora que ya se acerca el fin de este proceso, que ha sido tan duro y a la vez tan mágico, tengo el corazón lleno de ansiedad, emoción, alegría y gratitud. Muchas personas han estado a mi lado durante estos años, tanto presencialmente como vía internet, a las cuales me gustaría darles las gracias.

En primer lugar, a mis directoras de tesis las Dras. Teresa del Álamo Martín, Valle Flores Lucas y Begoña Coco Martín por las enseñanzas, paciencia y toda la dedicación dada a la realización de este trabajo. Teresa, contigo di mis primeros pasos por el camino de la investigación y empecé a construir una base sólida de aprendizaje, la cual llevaré conmigo por toda la vida. Gracias de todo mi corazón por darme una oportunidad, por apostar por mí, por toda atención, cariño, paciencia y amistad. Valle, de ti siempre recibí apoyo y fuerza para seguir adelante. Gracias por tus orientaciones, por animarme siempre en los momentos más duros, por preocuparte por mí, por tu sensibilidad y por creer en este trabajo. Begoña, aprendí muchísimo contigo. Tu participación en esta tesis fue fundamental para su realización. Gracias por guiarme siempre, por abrirme tantas puertas, por sacar lo mejor de mí, por tu apoyo, paciencia y cuidado. A las tres, que aprendí a respetar $y$ admirar, todo mí cariño y gratitud.

En segundo lugar, al IOBA que ha sido mi segundo hogar aquí en España. A todos los oftalmólogos y ópticos-optometristas que contribuyeron a la captación y evaluación de los participantes. En particular al prof. Dr. José Carlos Pastor Jimeno por sus enseñanzas, y a Rubén Cuadrado Asensio y José Alberto Lázaro por sus orientaciones, por la paciencia y por estar siempre disponibles a ayudar.

A la Asociación Castellano Leonesa de Afectados de Retinosis Pigmentaria, en particular al presidente Félix Román Barcenilla y al 
secretario José Luis San José por la generosidad, acogida y ayuda en la captación de participantes.

Al Centro de Personas Mayores Zona Sur, en especial a la directora Maria Luisa Centeno Tabarés por su esfuerzo, dedicación y cariño. Sin su ayuda nada de esto sería posible.

A todas las personas que aceptaron participar en este estudio de manera voluntaria y desinteresada. Gracias por vuestro tiempo, paciencia, cariño y por los momentos agradables y de muchos aprendizajes.

A la Dra. Itziar Fernández por su colaboración con la estadística y por su disponibilidad siempre.

A Raquel Sitonio, Marília Seabra, Patyanne y Pâmmela Carvalho, Luana Cavalcanti y a todos mis amigos por estar siempre a mi lado y por todas las palabras de incentivo en esta larga y dura caminada.

A Luis Ángel Vázquez, que estuvo a mí lado en todos estos años, animándome, levantándome en las caídas y aplaudiéndome a cada logro. Tenerte a mi lado hizo todo más llevadero y feliz. ;Te quiero;

A mi familia, titia, vovó, Dudu, Ricardo, mis sobrinos y mis cuñadas, gracias por vuestro apoyo, amor y por mantenerme siempre cerca a pesar de la distancia. ¡Os amo!

$Y$, por supuesto a mis padres. Vosotros sois mi fuente de inspiración, fuerza y amor. Las palabras no son suficientes para expresar mi gratitud por tanto amor y dedicación hacia mí. Muchísimas gracias por estar siempre presentes en mi vida. ¡Os amo!

Espero no haberme olvidado a nadie. De todo el corazón, muchas gracias a todos.

Ana Patrícia de Queiroz Barbosa 


\section{Lista de Acrónimos y Siglas}

RV: Rehabilitación Visual

BV: Baja Visión

CIE: Clasificación Internacional de Enfermedades

DMAE: Degeneración Macular Relacionada a la Edad

DV: Discapacidad Visual

AV: Agudeza Visual

SC: Sensibilidad al Contraste

CV: Campo Visual

C7: $7^{\text {a }}$ vértebra cervical

T12: $12^{\underline{a}}$ vértebra torácica

VL: Velocidad Lectora

IOBA: Instituto Universitario de Oftalmobiología Aplicada

ACLARP: Asociación Castellano-Leonesa de Afectados de Retinosis Pigmentaria SAPO: Software de Avaliação Postural

GDS: Geriatric Depression Scale

FES-I: Falls Efficacy Scale-International

EVA: Escala Visual Analógica

TUG: Timed Up and Go Test

IReST: New International Reading Speed Texts

FC: Flexión de la cabeza

FCu: Flexión del cuello

ACC: Ángulo cráneo-cervical

ACT: Ángulo cérvico-torácico

FT: Flexión torácica

AT: Ángulo del tronco 

AL: Ángulo lumbar
IP: Inclinación pélvica
ICC: Coeficiente de Correlación Intra-clase
CV: Coeficiente de Variación Intra-sujeto
DT: Desviación típica
IC: Intervalo de Confianza
IMC: Índice de masa corporal
ROM: Rango de movimiento
EIAS: Espina llíaca Antero-superior
ppm: Palabras por minuto 

La población mundial está envejeciendo, lo que implica un aumento de las enfermedades asociadas a la edad además de una mayor demanda de los servicios sanitarios, por lo que hay un interés creciente en investigaciones en esta población que redunden en conocimiento y programas que permitan retrasar y reducir las posibles situaciones de discapacidad y/o dependencia derivadas de las enfermedades asociadas con la edad ${ }^{1}$. En este sentido, se sabe, por ejemplo, que los problemas del sistema motor relacionados con los cambios en los sistemas nerviosos central y periférico y en el sistema musculoesquelético son parte del proceso normal de envejecimiento y pueden ser observados en algunos aspectos como la coordinación disminuida, movimientos lentos y dificultades con el equilibrio y la marcha ${ }^{2}$. Las funciones de destreza manual y sensibilidad táctil también están deterioradas en las personas mayores, lo que dificulta la realización de las tareas manuales ${ }^{3,4}$.

Otros problemas específicos del envejecimiento, además de los apuntados, son la disminución de la agudeza visual y auditiva, la incontinencia urinaria, una mayor incidencia de caídas, demencia, una mayor prevalencia de depresión y ansiedad y aislamiento social ${ }^{5}$. El proceso de envejecer trae consigo alteraciones físicas que por sí mismas ya son debilitantes y, si le añadimos la discapacidad visual (DV), dichas limitaciones pueden ser todavía más severas.

La rehabilitación visual $(\mathrm{RV})$ es una opción de tratamiento muy efectiva y que logra resultados muy positivos, dónde los pacientes refieren mejora en el aprovechamiento del resto visual y también en su calidad de vida ${ }^{6}$. Teniendo en cuenta que la lectura es una de las actividades más importantes para la realización de tareas cotidianas como ir a la compra, leer las etiquetas de los medicamentos, etc., y que la pérdida de dicha capacidad implica una restricción severa de la independencia y calidad de vida, por tanto, mejorar la capacidad lectora es uno de los principales objetivos de la RV en la mayoría de los $\operatorname{casos}^{7-9}$. Para lograr la recuperación o mantenimiento de la habilidad lectora, muchas veces es necesaria la prescripción de dispositivos ópticos y/o electrónicos. Sin embargo, debido a las limitaciones físicas inherentes al envejecimiento anteriormente mencionadas, muchos pacientes presentan dificultades a la hora de manejar dichos dispositivos, elemento considerado por algunos autores como limitante en las personas mayores ${ }^{10}$.

Muchos son los factores que pueden influir en la eficacia de un programa de $\mathrm{RV}$ para personas con baja visión (BV), principalmente en la habilidad lectora. Existen trabajos que describen algunos de ellos como, por ejemplo, los problemas visuales del individuo ${ }^{1-14}$ así como los factores emocionales y sociales ${ }^{15}$. Otro factor no visual que podría interferir en un programa de RV es la postura corporal adoptada en el momento de la lectura, si tenemos en cuenta que al reducir la distancia de trabajo para utilizar las ayudas ópticas, se adoptan posturas fatigosas que conducen a molestias músculoesqueléticas ${ }^{16,17}$. 
Existen estudios que hablan sobre las malas posturas adoptadas en la posición sentada al realizarse tareas de cerca, como la lectura y de qué manera la velocidad lectora se ve afectada por la postura corporal adoptada ${ }^{18-24}$. No obstante, las alteraciones posturales en personas mayores con BV todavía no han sido descritas ni tampoco su posible efecto en la aceptación y éxito de un programa de RV.

En este contexto parece justificado averiguar si:

- Existen diferencias posturales entre las personas mayores con y sin baja visión al realizar la lectura de un texto en sedestación.

- La alteración postural durante la lectura puede interferir negativamente en la velocidad lectora, dificultando su rendimiento. Además de otros factores como el equilibrio corporal, la función visual y el estado emocional.

- Es posible que un déficit en la destreza manual dificulte la utilización de ayudas ópticas para leer, disminuyendo la velocidad lectora.

A causa de estas dudas sentimos la necesidad y la curiosidad de investigar más a fondo sobre el tema, así que esta tesis doctoral pretende analizar si existen diferencias en cuanto a la aparición y/o severidad de las alteraciones posturales, de equilibrio y destreza manual a mayores con y sin BV. Además, si las alteraciones en la postura y equilibrio corporal y en la destreza manual existentes en las personas mayores con baja visión, interfieren de manera negativa en la velocidad lectora. Conjuntamente, también creemos importante determinar si tales alteraciones tienen algún efecto sobre el estado emocional del individuo.

Para llevar a cabo dicho estudio, se evaluó la postura y el equilibrio corporal y la destreza manual de personas mayores con y sin baja visión, además de su velocidad lectora con la ayuda óptica específica, en los casos que era necesario.

La evaluación postural fue llevada a cabo a través del uso de métodos cuantitativos, que para obtener resultados más precisos es necesario que las mediciones sean fiables. Con lo cual, se realizó una evaluación de la fiabilidad de los métodos de medición de la postura utilizados en este estudio.

A pesar de que los efectos psicológicos de la DV no son el objetivo principal de esta investigación, su estudio se planteó como objetivo secundario teniendo en cuenta su importancia para entender la DV en todos sus aspectos. Para ello, utilizamos cuestionarios específicos para la evaluación de la presencia de depresión, dolor autopercibido y miedo a caerse.

Esperamos que los resultados obtenidos en este trabajo nos permitan mejorar el conocimiento sobre las dificultades presentadas por las personas mayores con $\mathrm{BV}$ en el proceso de RV, para poder diseñar programas de rehabilitación específicos que disminuyan dichas dificultades. 
2. ANTECEDENTES Y ESTADO ACTUAL

DEL TEMA 



\subsection{Discapacidad visual y Baja visión}

La deficiencia visual puede ser definida como un problema en la función o estructura del sistema visual o del ojo(s) ${ }^{25}$ pudiendo ser identificada a través de diversas medidas clínicas, como la reducción de la agudeza visual o sensibilidad al contraste, pérdida de campo visual, fotofobia, diplopía o distorsión visual ${ }^{26}$. El surgimiento de dichas alteraciones puede deberse a factores congénitos, hereditarios o condiciones adquiridas.

Las medidas clínicas más tradicionales, como la agudeza visual, la sensibilidad al contraste y el campo visual, proporcionan informaciones muy limitadas cuando son realizadas de manera independiente. La agudeza visual (AV) es parte de la exploración oftalmológica básica aportando un dato importante en la historia clínica del paciente para la determinación de la refracción exacta necesaria para optimizarla. Se define como, "la capacidad que posee el ojo de distinguir los más pequeños detalles de un objeto"27. Las pruebas utilizadas en la práctica clínica para medir la AV constituyen, básicamente, la discriminación de letras y números. La exploración debe hacerse en cada ojo por separado, tanto de lejos como de cerca. Para la visión lejana se utilizan los optotipos específicos que consisten en letras, números, dibujos o signos, de acuerdo con la formación intelectual y la edad del paciente. Y, para la visión de cerca existen cartillas específicas con textos o signos de diferentes tamaños ${ }^{28}$.

La reducción de la AV puede dificultar la realización de tareas de la vida cotidiana, desplazamiento, lectura, entre otras. Pero dichas dificultades también pueden presentarse en personas con problemas para diferenciar detalles, es decir, para percibir los diferentes contrastes. La evaluación de la sensibilidad al contraste (SC) tiene el objetivo de medir la habilidad del sistema visual para distinguir entre un objeto y el fondo sobre el cual está (Figura 1A), proporcionando información respecto a la calidad visual ${ }^{29}$.

Por su parte, el campo visual (CV) se refiere a la visión periférica, así que es el espacio perceptible en que el ojo, en posición fija, percibe la luz, la forma y los colores (Figura 1B y 1C). El CV normal en el adulto sano se extiende hasta $180^{\circ}$ en el eje horizontal y $120^{\circ}$ en el eje vertical ${ }^{30}$.

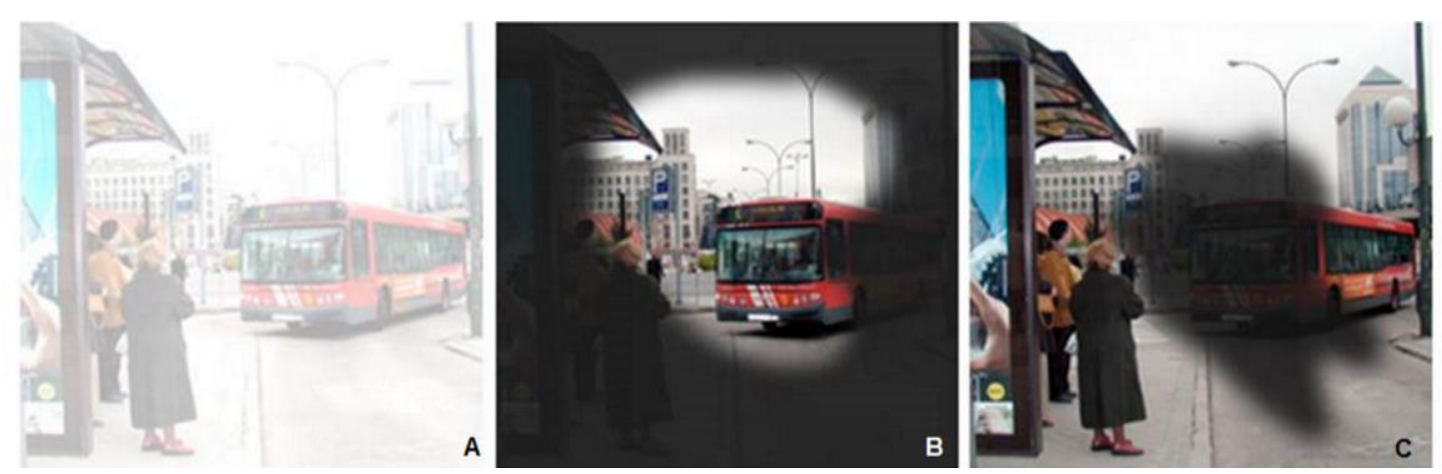

Figura 1: A. Disminución de la SC. B. Pérdida de CV periférico. C. Pérdida de CV central. Fuente: http://www.baja-vision.org/patologias.htm 
En la literatura existen diferentes definiciones para las terminologías relacionadas con la DV y la $\mathrm{BV}$, lo que ha dificultado el conocimiento de su magnitud e impacto reales en el mundo. Hasta el día de hoy no hay parámetros estandarizados adoptados unánimemente por la comunidad internacional para definir la DV y la BV. El término "Discapacidad Visual" a menudo se utiliza de manera inconsistente, dado que es empleado tanto para las personas que tienen visión parcial, como en lo que se refiere a la ceguera ${ }^{31}$. Sin embargo, la categorización de la DV, según sus niveles de deterioro, utilizada con mayor frecuencia para las estimaciones mundiales es la adoptada por la Clasificación Internacional de Enfermedades (CIE) ${ }^{32}$.

La CIE, en su $10^{\text {a }}$ revisión, dispone la definición respecto a los grados de la DV en categorías según la $A V$ presentada por el sujeto, que debería medirse con los dos ojos abiertos y con la corrección adecuada, si fuera necesaria. La BV, término utilizado en este estudio, corresponde a las discapacidades visuales moderada y severa. En la Tabla 1 es posible visualizar todas las categorías que integran el término "discapacidad visual".

Tabla 1: Clasificación de la gravedad de la discapacidad visual.

\begin{tabular}{|c|c|c|}
\hline \multirow[t]{2}{*}{ Categorías } & \multicolumn{2}{|c|}{ Agudeza visual de lejos } \\
\hline & Peor que: & Igual o mejor que: \\
\hline $\begin{array}{l}0 \text { Discapacidad Visual } \\
\text { Leve o ninguna }\end{array}$ & & $\begin{array}{c}6 / 18 \\
3 / 10(0.3) \\
20 / 70\end{array}$ \\
\hline $\begin{array}{c}1 \text { Discapacidad Visual } \\
\text { Moderada }\end{array}$ & $\begin{array}{c}6 / 18 \\
3 / 10(0.3) \\
20 / 70\end{array}$ & $\begin{array}{c}6 / 60 \\
1 / 10(0.1) \\
20 / 200\end{array}$ \\
\hline $\begin{array}{c}2 \text { Discapacidad Visual } \\
\text { Severa }\end{array}$ & $\begin{array}{c}6 / 60 \\
1 / 10(0.1) \\
20 / 200\end{array}$ & $\begin{array}{c}3 / 60 \\
1 / 20(0.05) \\
20 / 400\end{array}$ \\
\hline 3 Ceguera & $\begin{array}{c}3 / 60 \\
1 / 20(0.05) \\
20 / 400\end{array}$ & $\begin{array}{c}1 / 60^{*} \\
1 / 50(0.02) \\
5 / 300(20 / 1200)\end{array}$ \\
\hline 4 Ceguera & $\begin{array}{c}1 / 60^{\star} \\
1 / 50(0.02) \\
5 / 300(20 / 1200) \\
\end{array}$ & Percepción de la luz \\
\hline 5 Ceguera & No percepción de la luz & \\
\hline 9 & $\begin{array}{l}\text { Indeterminado o no } \\
\text { especificado } \\
\text { *o recuentos de dedos a } \\
1 \text { metro }\end{array}$ & \\
\hline
\end{tabular}

La CIE-10 está considerada como la clasificación estándar en todo el mundo y es la más utilizada para realizar estimaciones mundiales de DV. Esta clasificación incluye el criterio de campo visual solamente en la definición de la ceguera, excluyendo dicho criterio en las categorías 1 y 2 . Así que, conforme a 
tal concepto, forman parte de la categoría 3 las personas con campo visual en el mejor ojo menor que $10^{\circ}$ alrededor de la fijación central ${ }^{33}$. En lo que se refiere a las categorías 1 y 2 , hay estudios que utilizan el campo visual menor que $20^{\circ}$ como criterio de discapacidad visual equivalente a agudeza visual menor que $6 / 60^{32,34,35}$.

La CIE-10 se utiliza para hacer el diagnóstico cuantitativo de la DV. Para una evaluación completa, dicha clasificación no es suficiente. Es necesario completarla con otras clasificaciones. Debido a la necesidad de obtener una clasificación que retratase la importancia del contexto social en la producción de la discapacidad, la OMS adoptó en mayo de 2001 la nueva Clasificación Internacional del Funcionamiento, de la Discapacidad y de la Salud (CIF). La misma actúa como un instrumento diseñado para describir y medir la salud y la discapacidad. Se establece como una clasificación de los componentes de la salud y no de las consecuencias de la enfermedad, y se recomienda que sea utilizada de forma complementaria, junto con la CIE-10 ${ }^{36}$.

Mediante la CIF es posible describir un perfil del funcionamiento humano, es decir, detallar el estado de salud en que se encuentra una persona, basado en sus 4 componentes: funciones corporales, estructuras corporales, actividad y participación y factores ambientales ${ }^{37}$.

Dado que la CIF clasifica no sólo la salud sino también a los estados relacionados con ella, es extensamente utilizada en todo el mundo sirviendo de base para varios estudios epidemiológicos, para elaborar diferentes modelos de rehabilitación y de actuaciones en el campo de la provisión de servicios para las personas con discapacidad. Además, también ha sido adoptada por diversos sectores, tales como seguros, seguridad social, trabajo, educación, economía, política social, desarrollo de las políticas y la legislación en general y los cambios ambientales. Esto hace que sea un instrumento con enfoque multidisciplinario, estableciendo un lenguaje común de las condiciones relacionadas con la salud y permitiendo la comparación de datos entre países y entre servicios en diferentes momentos en el tiempo ${ }^{25,36}$.

Con lo cual se puede considerar que el concepto de DV engloba las deficiencias visuales, limitaciones en las actividades o restricciones en la participación ${ }^{36}$. Lo que concuerda con la definición adoptada por el National Eye Institute ${ }^{38}$ que considera otros factores además de los meramente visuales a la hora de definir la BV, siendo esta una DV no corregible con gafas normales, lentes de contacto, medicamentos o cirugía y que interfiere con la capacidad para realizar actividades de la vida diaria, limitando la independencia personal o socioeconómica. 


\subsection{La Columna Vertebral: Anatomía y Biomecánica}

La columna vertebral, también denominada raquis, es conocida como el pilar de sustentación óseo del cuerpo. Está compuesta por dos tipos de elementos, las vértebras y los discos intervertebrales. Situada en la línea media posterior del tronco, posee una estructura compuesta por 33-34 vértebras superpuestas intercaladas con los discos intervertebrales, que además de soportar las cargas del cuerpo, protege las estructuras del sistema nervioso central y confiere rango de movimiento para los principales movimientos del tronco ${ }^{39-41}$ (Figura 2).

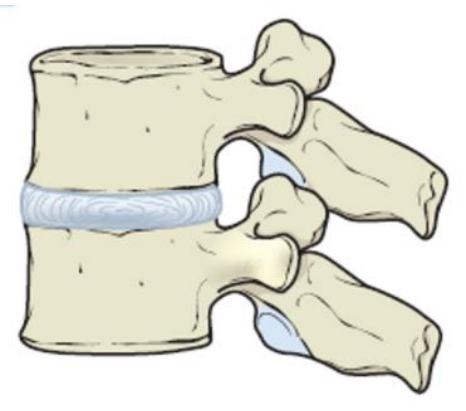

Figura 2: Unidad funcional normal de la columna vertebral. Fuente: Houglum e Bertoti ${ }^{42}$.

Está dividida en cuatro estructuras principales, las cuales presentan curvaturas de naturaleza fisiológica específicas que van sufriendo cambios en las diferentes etapas de la vida (Figura 3). La columna cervical, compuesta por 7 vértebras ( $\mathrm{C} 1 \mathrm{a} \mathrm{C} 7)$, presenta una curvatura de convexidad anterior; la columna torácica o dorsal, formada por 12 vértebras (T1 a T12), exhibe una curvatura de convexidad posterior; la columna lumbar, constituida por 5 vértebras (L1 a L5), luce una curvatura de convexidad anterior; la columna sacra, constituida por 5 vértebras (S1 a S5) agrupadas formando el sacro, posee una curvatura de convexidad posterior; y, finalmente, la coccígea, formada por 4 o 5 vértebras que constituyen el cóccix ${ }^{39-41,43}$. De éstas, la cervical y lumbar son las más móviles, mientras la torácica es más rígida, aportando menor movilidad. 

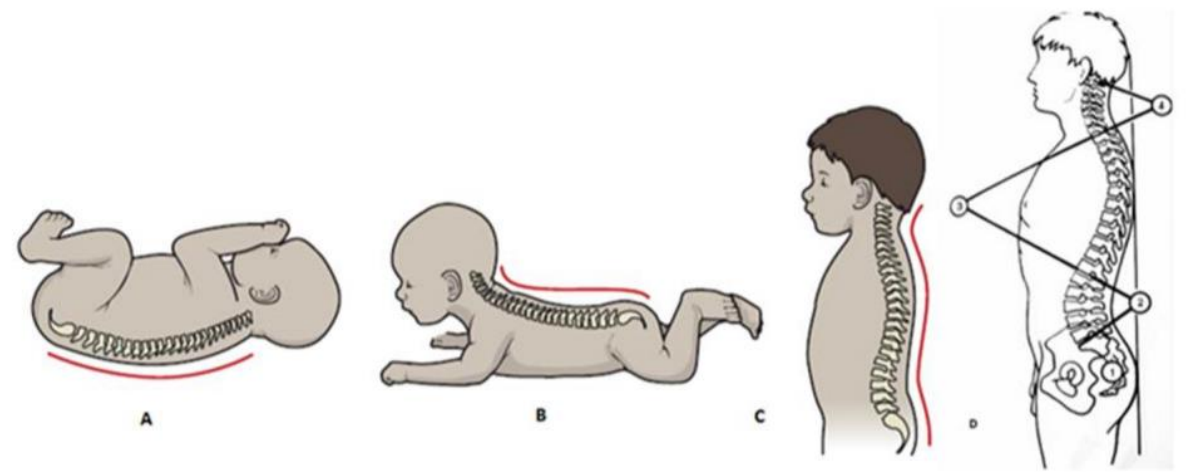

Figura 3: Cambios en las curvaturas vertebrales. A. Curvatura convexa posterior. B. Desarrollo de la lordosis cervical. C. Alrededor de los 10 años de edad, todas las curvaturas fisiológicas son iguales a las de un adulto. D. Curvaturas de la columna vertebral.

Fuente: Houglum e Bertoti ${ }^{42}$; Ocampo Fonseca et al. ${ }^{44}$.

Cuando la columna sufre regularmente demasiadas fuerzas asimétricas, dichas curvaturas pueden alterarse llevando a diferentes posturas patológicas (Figura 4). En el momento en que estas alteraciones están instaladas, es posible identificar cambios en la alineación de la columna vertebral del segmento afectado, los cuales habitualmente están directamente relacionados a la magnitud de las fuerzas que actúan sobre la misma. Dichos cambios pueden ser influenciados por diversos factores como: determinadas condiciones patológicas, el estado mental del individuo, enfermedades congénitas de la columna, debilidad de los músculos abdominales, obesidad, osteoporosis, presencia de dolor en alguna parte del cuerpo, la edad y la postura corporal adoptada.
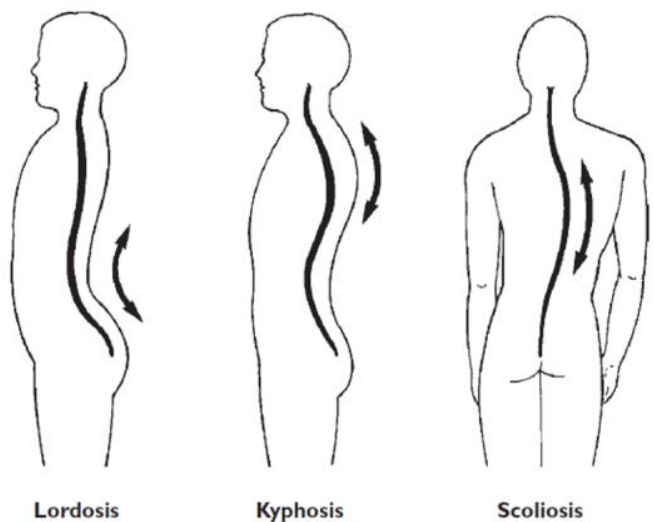

Figura 4: Curvaturas anormales de la columna. Fuente: Hall, Susan ${ }^{45}$.

Con el avance de la edad, generalmente después de los 50 años empiezan a producirse algunos cambios naturales en la alineación de la columna vertebral. Comparados con adultos jóvenes, las personas mayores presentan alteraciones en la alineación de la columna lumbar resultantes de la 
disminución en la altura de los discos intervertebrales y del aumento de la dimensión de los mismos ${ }^{46}$. Con lo cual conduce a una disminución de la lordosis lumbar, que es más acentuada en los hombres que en las mujeres ${ }^{47}$. La disminución de la lordosis lumbar ocurre cuando la reducción del disco intervertebral es en la región anterior, lo que produce alteraciones compensatorias en la postura corporal|48,49.

\subsection{Postura corporal}

En la actualidad existen múltiples definiciones para el término postura corporal. Mannheimer y Rosenthal ${ }^{50}$ decían que la postura corporal depende de la relación entre la actividad de los músculos anterior y posterior y el ajuste fisiológico conveniente a los aspectos físicos y emocionales de la vida diaria. Ya Snell ${ }^{51}$ defendía que la postura depende del tono muscular del individuo, lo cual condiciona la posición adoptada por él en su medio ambiente. Algunos autores definen la postura como una posición, actitud o disposición relativa de las partes del cuerpo para una actividad específica o una forma característica de sujetar el propio cuerpo ${ }^{42,52}$. Stanos, McLean y Rader ${ }^{53}$ afirman que la postura está directamente influenciada por cada una de las articulaciones, es decir, cuando una persona adopta una mala postura se producen tensiones anormales en las mismas, mientras que cuando éstas están alineadas el estrés muscular es mínimo y se logra una postura correcta. Así que, cuando hay un equilibrio entre las articulaciones y los tejidos a su alrededor disminuyendo la carga articular, la postura es neutra y es la considerada ideal ${ }^{54,55}$.

La posición deja de ser neutra en el momento en que se inicia cualquier movimiento, pudiendo recuperarse cuando finaliza dicho movimiento. Lo que significa que la duración y la frecuencia con que son adoptadas las posturas estáticas son determinantes en la proporción del daño causado, llegando a afectar la eficiencia muscular y generando condiciones musculoesqueléticas patológicas ${ }^{55-57}$.

Tal hecho ocurre porque al realizar un movimiento los músculos actúan en combinación, a un lado unos realizan la contracción (agonistas) y al lado opuesto otros están relajados (antagonistas) manteniendo el equilibrio muscular. Por ejemplo, durante la flexión del brazo, además de los músculos que producen la flexión participan también del movimiento los músculos posteriores con el fin de soportar la carga y mantener la posición (Figura 5). 

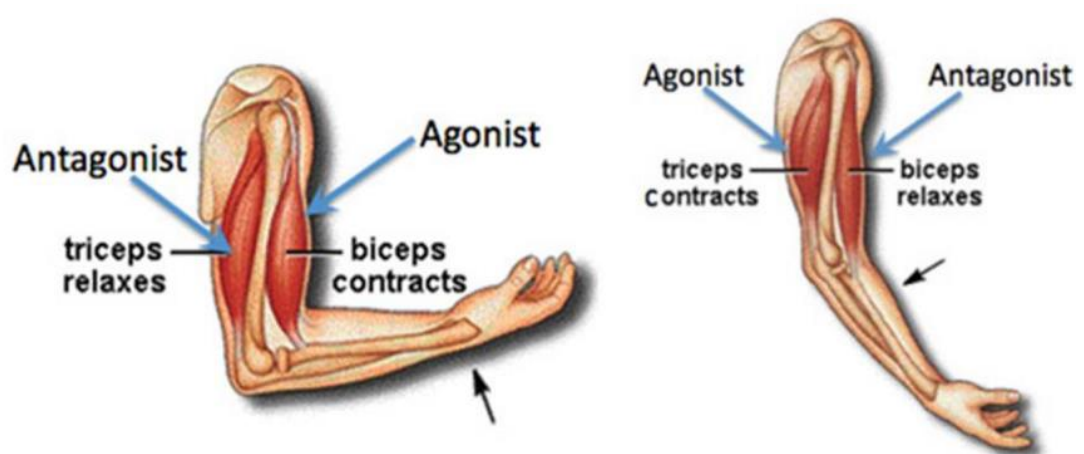

Figura 5: Músculos agonista y antagonista para la flexión del brazo. Fuente: http://shywmobile.com/author/lucille/page/3/

Con la repetición constante y/o el mantenimiento de dicha postura el músculo agonista adopta una posición acortada, siendo este músculo generalmente más fuerte en comparación con su antagonista, alargado y débilis. Este principio descrito es similar para la columna, es decir, al realizar el movimiento de flexión o extensión de la misma se produce una fuerza de compresión en un lado del disco intervertebral y una fuerza de tensión en el otro (Figura 6).

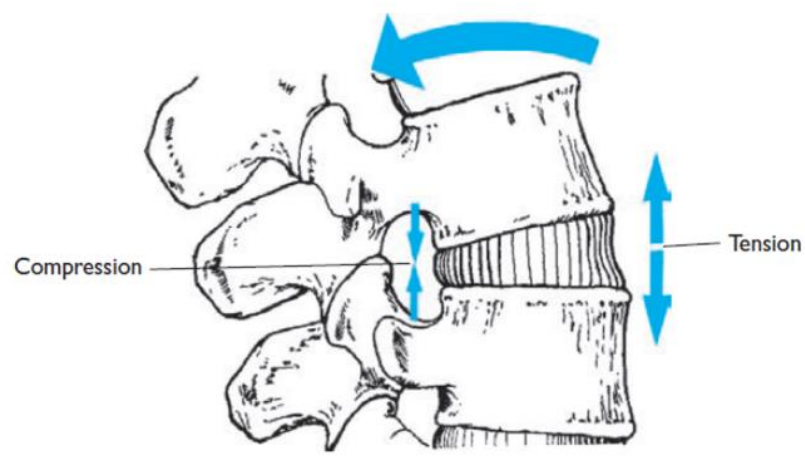

Figura 6: Fuerzas de compresión y tensión en el disco intervertebral. Fuente: Hall, Susan ${ }^{45}$

Con lo cual se puede decir que los movimientos corporales ocurren de manera integrada y compensatoria y que, en condiciones normales, llevan a una condición de equilibrio. Sin embargo, cuando se produce un desequilibrio surgen las alteraciones funcionales ${ }^{52}$.

Con el proceso de degeneración de los discos intervertebrales en algunos segmentos de la columna, otras estructuras (facetas y cápsulas articulares) asumen las cargas de los discos llevando a un desequilibrio. Con lo cual, la postura en bipedestación podría verse alterada ya que al intentar aliviar la compresión en las articulaciones se adopta una postura de flexión del tronco, reduciendo la lordosis lumbar ${ }^{42,45}$. 
En lo que se refiere a la postura sentada, las curvaturas fisiológicas de la columna son menos perceptibles, pudiendo sufrir cambios de acuerdo con la postura adoptada por el individuo. La literatura hace referencia a tres posturas sentadas específicas. La primera sería con la columna torácica en vertical (Figura 7A), que se caracteriza por el aumento de la lordosis torácica, disminución de la lordosis lumbar y menor inclinación pélvica anterior. La segunda y más comúnmente adoptada es la muy inclinada, es decir, con la rotación posterior de la pelvis y flexión tóraco-lumbar (Figura 7B). Es conocida como una postura pasiva por resultar en la disminución en la activación de los músculos antigravitatorios. La tercera es la columna lumbar en vertical (Figura 7C), donde hay una rotación pélvica anterior, una lordosis lumbar neutra y la columna torácica relajada ${ }^{59,60}$.

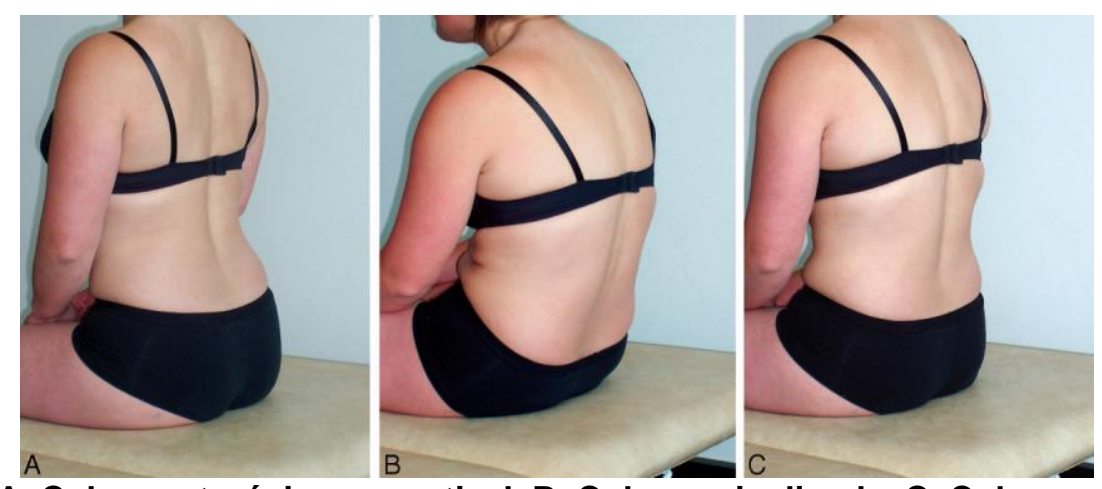

Figura 7: A. Columna torácica en vertical. B. Columna inclinada. C. Columna lumbar en vertical.

Fuente: O'Sullivan et al., $2006^{60}$.

Parece ser que la postura sentada con la columna lumbar en vertical es la que más se acerca a la postura óptima, sin embargo la literatura ergonómica es confusa y contradictoria. Muchos autores afirman que entra las posturas lordótica (Figura 7A) y cifótica (Figura 7B), la primera sería la menos dañina para la salud de la columna vertebral|61-64. No obstante, el diseño de la mayoría de los asientos utilizados para actividades de ocio a menudo invitan a posturas cifóticas.

\subsubsection{Postura Corporal y Baja Visión}

La ausencia o la restricción del sentido de la vista limitan la exploración del ambiente, influenciando negativamente el desarrollo del equilibrio, de la coordinación motora y del control y postura corporales. Lo que conduce a la pérdida de equilibrio y movilidad y, consecuentemente, al miedo a caerse y limitación de la independencia ${ }^{2,65-67}$.

De hecho, algunos estudios han relacionado la DV con la alteración postural, principalmente en niños y adultos jóvenes con ceguera congénita ${ }^{68-71}$. Las 
principales alteraciones posturales mencionadas en dichos estudios fueron: cabeza hacia delante, escoliosis, asimetría de los hombros, prominencia abdominal y rotación anterior de la pelvis.

El hecho de que las personas con BV presentan un concepto inadecuado sobre la posición vertical, una autoimagen corporal limitada e hiper e hipotonía de los músculos posturales por el intento de compensar la falta de la visión, son algunas de las posibles causas por las cuales dichas personas adoptan las malas posturas ${ }^{72}$.

La postura anormal de la cabeza, también conocida como postura compensatoria, a menudo se ve relacionada con la vista. El movimiento de la cabeza es un intento de optimizar la agudeza visual, considerando que al moverla hacia un lado y hacia el otro, hacia arriba o abajo, los ojos giran en dirección opuesta para compensar el movimiento (Figura 8) ) $^{73,74}$.

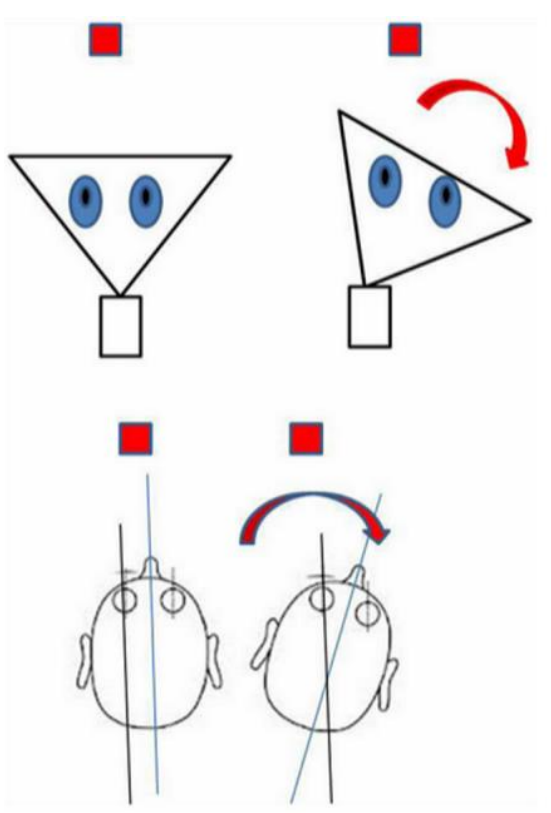

Figura 8: Reflejo vestíbulo-ocular lineal.

Fuente: Castillo y Jorge ${ }^{74}$.

Las alteraciones posturales presentes en otros segmentos del cuerpo, mencionadas anteriormente, pueden estar relacionadas con el posicionamiento compensatorio de la cabeza. Puesto que, en la posición estática, la postura de cada segmento de la columna está relacionada. Es decir, la alteración de la postura en una determinada región de la columna puede alterar la postura en otro segmento. Por ejemplo, una postura de la cabeza hacia delante viene acompañada por una flexión de la región cervical inferior y una extensión de la región cervical superior ${ }^{52,75-77}$ (Figura 9). 

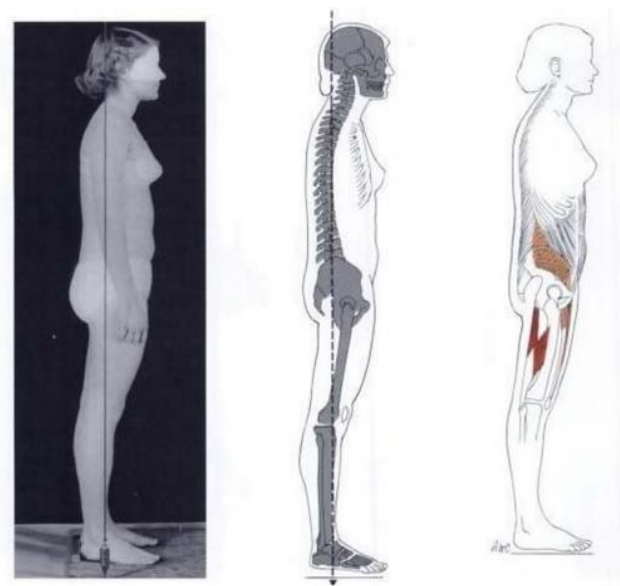

Figura 9: Relación entre la posición de la cabeza y la columna cervical.

Fuente: Kendall, $2000^{52}$.

La mayoría de los estudios sobre la postura corporal en las personas con DV están centrados en las posturas de pie con información limitada sobre las posturas sentadas. La cantidad de tiempo que los adultos pasan sentados durante el día es de 5 a 6 horas $^{78}$. Este hecho merece atención teniendo en cuenta que estar sentado solamente durante un período de dos horas al día es suficiente para causar altas cargas de compresión en las articulaciones de la columna vertebral lumbar, debido a la sobrecarga constante asociada con la posición estática ${ }^{79}$. Harrison et al. ${ }^{80}$ llegaron a la conclusión de que cuando un sujeto se mantiene sentado durante un tiempo prolongado, a veces se observa un incremento progresivo de la flexión lumbar y esto podría ser debido a algunos factores como: la sensación de comodidad, la práctica habitual y el diseño de la silla, por ejemplo, altura y ángulo del respaldo y asiento y presencia de los apoyabrazos ${ }^{20}$. Existe una estrecha relación entre el tiempo prolongado en la posición sentada y el dolor de espalda, y, probablemente, esta asociación se atribuye a la flexión de la columna lumbar comúnmente adoptada para mantener esa postura ${ }^{81-83}$.

Para facilitar la lectura, una persona con DV tienen que reducir la distancia del texto, moviendo el material de lectura más cerca del ojo o acercándose a la mesa (Figura 10) ${ }^{84}$. La reducción de la distancia cercana de trabajo y el uso de dispositivos de aumento ópticos, conduce a las posturas fatigosas y molestias musculoesqueléticas ${ }^{16,17}$. 


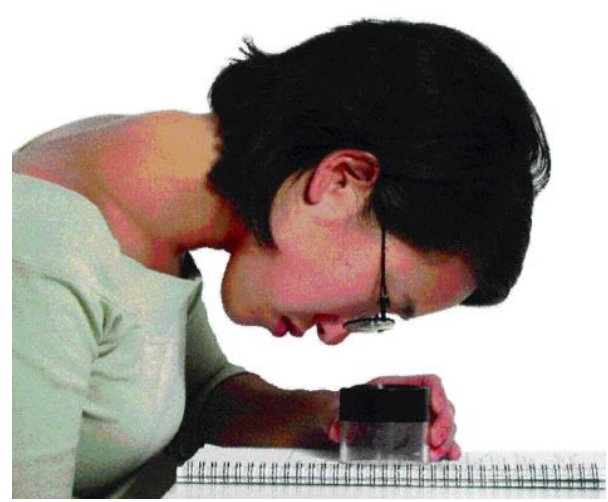

Figura 10: Distancia de trabajo con ayudas ópticas.

Fuente: Minto y Butt, $2004^{83}$.

Poco se sabe sobre la salud musculoesquelética en las personas con BV, sin embargo existe una relación entre la BV y el dolor de espalda en las personas mayores ${ }^{85}$. A pesar de los escritos existentes, son necesarias más investigaciones acerca de la postura corporal, principalmente en personas mayores con BV.

\subsubsection{Métodos de Evaluación Postural}

Una manera de saber si los segmentos corporales de una persona están alineados o no es a través de la evaluación de la postura corporal. Muchos profesionales de la salud utilizan la evaluación postural como un método para valorar inicialmente al paciente y comparar los resultados obtenidos durante y tras la finalización del tratamiento. En la literatura actual es posible encontrar diversos métodos de evaluación de la postura. Entre los más comunes destacamos ${ }^{86}$ :

$\checkmark$ Plataforma de fuerza: es un método generalmente utilizado para evaluar el equilibrio y el control postural a través del centro de presión (CP), entendido como el punto resultante de la acción de la fuerza vertical sobre una superficie. Consiste en un tablero equipado con sensores de movimiento que registran los movimientos anterior-posterior y mediallateral del sujeto ${ }^{87}$. En sujetos sanos, el CP está localizado posteriormente, más cerca del tobillo, a la mitad de la base de sustentación (es decir, los pies) ${ }^{88}$. Algunos estudios refieren su uso para diferenciar personas con riesgo 0 no de caerse ${ }^{89,90}$, evaluar la estabilidad postura ${ }^{91}$ así como para valorar las deficiencias de equilibrio causadas por factores relacionados con algunas enfermedades ${ }^{92-98}$.

$\checkmark$ Goniómetro: es una herramienta común en la práctica clínica cuando se quiere verificar el rango de movimiento articular y su resultado es reproducido en ángulos. Sin embargo, algunos estudios recomiendan su utilización apenas en ciertas articulaciones y no para una evaluación global de la postura ya que se tardaría mucho en hacerlo y sería 
necesario total colaboración del paciente en una posición estática (cualquier movimiento puede alterar el resultado tornando la medida no fiable $)^{86,99}$.

$\checkmark$ Rayos X: es el procedimiento estándar para medir objetivamente la postura a partir de los puntos de referencia óseos, pero su utilización en la práctica clínica y en investigación es limitada por su alto costo y por los peligros de la exposición a la radiación ${ }^{86,100-103}$.

$\checkmark$ Imágenes por Resonancia Magnética (IRM) y Tomografía Computarizada (TC): ambas técnicas son empleadas para conocer la geometría y posición óseas produciendo imágenes de alta calidad en 3D. Las ventajas de la IRM sobre la TC es que la primera no es invasiva, permite escanear imágenes en diversas posturas y tiene una alta precisión diagnóstica ${ }^{104-106}$.

$\checkmark$ Sensores biomédicos: son dispositivos compactos, portátiles, discretos y confiables, diseñados para detectar, monitorear y reunir datos del movimiento humano. Pueden ser llevados puestos en muchas partes del cuerpo como la cintura o muñecas y pueden ser goniómetros, sensores giroscópicos, inclinómetros, sensores de fuerza, acelerómetros, entre otros ${ }^{107}$. Los acelerómetros se han convertido en el método preferido de detección de movimiento y monitoreo humano por ser continuo, discreto y confiable, siendo también utilizado para medir la postura del cuerpo ${ }^{86}$.

$\checkmark$ Fotogrametría: es considerado fiable para evaluar la alineación postural, además de ser una herramienta eficaz y segura en la evaluación, análisis y cuantificación de los cambios posturales. Para realizar el análisis fotogramétrico, algunos puntos anatómicos son marcados y luego se hacen los registros fotográficos ${ }^{108-110}$. Entre las limitaciones de esta técnica señalamos que las medidas son externas al cuerpo, es decir, se utilizan los cálculos de las marcas óseas externas para estimar la postura lo que puede no dar una interpretación exacta de la verdadera alineación de la columna. Además, la falta de estandarización de las referencias anatómicas y los ángulos obtenidos entre ellas dificulta la comparación entre los estudios y la fiabilidad de los resultados ${ }^{111,112}$.

La definición de los ángulos posturales se hace a través de la elección de los puntos anatómicos, para los cuales no hay una estandarización por lo que varían de un estudio a otro. Las medidas posturales utilizadas son muchas e, incluso para el mismo segmento corporal puede existir más de una medida. Algunas de ellas son:

- Flexión de la cabeza con respecto a la vertical/horizontal:

- Trago, ojo y línea vertical a través de la oreja ${ }^{113}$.

- Ojo-oreja y línea horizontal a través de la oreja ${ }^{114-116 .}$

- Flexión del cuello con respecto a la vertical/horizontal: 
- Trago, C7 y línea vertical a través de $C 7^{113,116}$.

- Trago, apófisis del acromion y línea horizontal a través de la apófisis del acromion ${ }^{117}$.

- Ángulo cráneo-cervical:

- Ojo-trago-C7 $7^{113-115 .}$

- Ojo-oreja-T1118.

- Ángulo cérvico-torácico:

- Trago-C7-T5 ${ }^{113}$.

- Oreja-C7-cadera' ${ }^{114,115 .}$

- Flexión del tronco con respecto a la vertical/horizontal:

- C7-trocánter mayor y la línea vertical a través de trocánter mayor ${ }^{113,116}$.

- C7-trocánter mayor y la línea horizontal115.

- Ángulo del tronco:

- C7-T12- trocánter mayor 22,119.

- Ángulo lumbar:

- T12-espina ilíaca antero-superior (EIAS) y la línea de EIAS-trocánter mayor (ángulo posterior) ${ }^{21,22}$.

- Inclinación pélvica:

- Trocánter mayor-EIAS y la línea vertical a través del trocánter mayor ${ }^{21,22 .}$

\subsection{Equilibrio corporal}

El equilibrio, según Knudson ${ }^{120}$ es la capacidad que una persona posee para controlar la posición de su cuerpo en relación con alguna base de apoyo, es decir, es el control de la estabilidad y la movilidad del cuerpo. Dicha capacidad puede ser influenciada por muchos órganos sensoriales y procesos cognitivos que están implicados en tal proceso.

El equilibrio corporal es crucial tanto para la posición estática como en el movimiento dinámico ${ }^{120}$. Para alcanzarlo durante las actividades estáticas y dinámicas, el cuerpo utiliza diferentes sistemas. El sistema visual, así como el somatosensorial y el vestibular son los responsables por la construcción del equilibrio corporal. Este puede ser definido como la capacidad de mantener el centro de gravedad del cuerpo dentro de los límites de la base de apoyo y puede ser clasificado en estático y dinámico. El equilibrio corporal en posición 
vertical, con el individuo parado de pie o sentado es definido como equilibrio estático, mientras que cuando el centro de gravedad y/o la base de apoyo se están moviendo es conocido como equilibrio dinámico ${ }^{121}$.

Para mantenerse en equilibrio, el cuerpo tiene que adoptar diferentes estrategias de movimiento y control postural para compensar las perturbaciones externas. Los patrones de movimiento realizados para lograr tal control son categorizados en dos estrategias: la del tobillo (control postural antero-posterior) y la de la cadera (control postural medio-lateral). En ambas estrategias el cuerpo se mueve principalmente alrededor de la articulación (del tobillo o cadera) moviendo el centro de masa corporal hacia atrás o hacia adelante con el fin de restaurar el equilibrio ${ }^{122,123}$.

La elección por una u otra estrategia depende de algunos factores como las características del aparato locomotor, la información sensorial, la base de apoyo, limitaciones en la ejecución de algunas tareas y la amplitud de movimiento. Horak et al. ${ }^{124}$ reflejan en su estudio el papel de las informaciones somatosensorial y vestibular en la estrategia utilizada para mantenerse en equilibrio. La pérdida somatosensorial supone un aumento de la estrategia de la cadera y el déficit vestibular la del tobillo, con ausencia de la estrategia de la cadera.

Otro factor importante que influencia el equilibrio es la edad. Las personas mayores presentan limitaciones en el aparato locomotor relacionadas con los cambios en el sistema nervioso central, sistema nervioso periférico y en el sistema músculo-esquelético. Tales limitaciones pueden ser observadas en algunos aspectos como la coordinación disminuida, movimientos más lentos y dificultades con el equilibrio y la marcha ${ }^{2}$. Con lo cual, dichas personas empiezan a fiarse más del sentido de la vista para ayudar a mantener la estabilidad postural.

Comparados con adultos jóvenes, las personas mayores presentan diferentes estrategias de control postural al realizar alguna tarea específica (por ejemplo, la estrategia de la cadera), menor equilibrio corporal, además de una mayor utilización de la musculatura antagonista (músculos posteriores de la columna) y de secuencias musculares no vistas en los adultos jóvenes ${ }^{125-127}$.

Teniendo en cuenta que por el déficit locomotor, resultado del proceso normal del envejecimiento, las personas mayores suelen confiar en su sistema visual, al sufrir cualquier alteración en dicho sistema el equilibrio será afectado. De hecho, algunos estudios investigaron la relación entre la DV y el equilibrio, concluyendo que los adultos mayores con DV presentan menos estabilidad corporal cuando comparados a los adultos mayores sin DV67,128,129.

Los problemas de equilibrio junto con el deterioro visual han sido señalados como factores de riesgo para diversas disfunciones físicas y psicosociales. Por ejemplo, Ivers et al. ${ }^{130}$ mostraron una fuerte relación entre la DV y el riesgo de 
fractura de cadera. Dicho riesgo fue $40 \%$ más alto en individuos con mala AV. Lo que significa que las personas mayores con pobres funciones visuales son más propensas a caer y, en consecuencia, sufrir fracturas por la caída, que las personas mayores sin DV.

El desempeño de todas las actividades de la vida diaria requiere un buen equilibrio sea en reposo o durante el movimiento. Existen diversos tests para evaluar el equilibrio y muchos de ellos son baratos, de rápida ejecución y fiables. En un estudio realizado por Langley y Mackintosh ${ }^{131}$ con el fin de identificar la fiabilidad de los test de equilibrio utilizados en personas mayores, la escala de equilibrio de Berg y Timed up and Go fueron las pruebas más rigurosas y consideradas fiables y válidas para utilización con personas mayores.

\subsection{La lectura y el sistema visual}

El sistema lector está formado por diversos elementos que se han obtenido durante los primeros años de la escolaridad y que a lo largo del tiempo se han ido perfeccionando. En los adultos este sistema funciona como un mecanismo que nos permite traducir de una forma rápida y automática los signos escritos sobre el papel en significados.

Realizar una lectura comprensiva es una tarea muy compleja, para la cual es necesario realizar un elevado número de tareas cognitivas. Sin embargo, con la práctica, la mayoría de estas tareas se automatizan aportando mayor habilidad a la hora de leer. Prueba de ello es la velocidad con que lo hacemos (entre 150 y 400 palabras por minuto, ppm) $)^{132}$.

Sin embargo, cuando falla alguno de los componentes cognitivos necesarios para su realización como la percepción visual, resultado de una patología visual por ejemplo, la lectura deja de ser una tarea ágil y fluente para convertirse en algo difícil y extremadamente agotador.

En el proceso del desarrollo de la lectura puede ser dividido en cuatro niveles:

1. Perceptivo y de identificación de las letras. Se trata de la capacidad de reconocer las palabras, es decir identificar las letras de forma aislada y después formando las palabras. En esta fase el sistema visual tiene un papel fundamental que es el de descifrar los signos gráficos que son proyectados sobre nuestro cerebro. Para ello, los ojos realizan una serie de movimientos avanzando a pequeños saltos de izquierda a derecha, llamados movimientos sacádicos, que se alternan con periodos de fijación en los que permanecen inmóviles ${ }^{132}$. Durante el periodo de fijación, el lector es capaz de obtener información de una región que se extiende desde el comienzo de la palabra fijada (pero no más de 3 caracteres a la izquierda de la fijación) hasta aproximadamente 15 caracteres a la derecha de la misma ${ }^{133}$. El número y la duración de los 
momentos de fijación que ejecuta una persona es lo que establece su capacidad lectora. Al mismo tiempo en que se inicia la fijación empieza la extracción de la información. Su duración va de acuerdo con el tamaño de la palabra ya que el procesamiento se realiza letra a letra, de manera seguida, de izquierda a derecha, y a una velocidad de 10 a 20 mseg aproximadamente por letra ${ }^{134}$. Además de la fijación, otros aspectos son importantes para el proceso de lectura como el movimiento ocular de regresión, entendido como el movimiento de los ojos de derecha a izquierda, y el movimiento de retorno, en lo cual se realiza el cambio de una línea a otra (Figura 11) ${ }^{135}$.

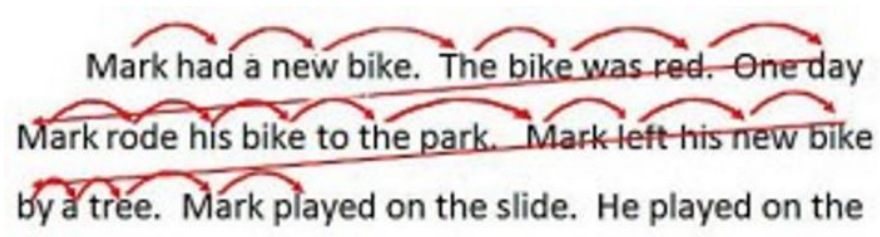

Figura 11: Movimientos oculares precisos a lo largo de la línea y retorno al principio de la siguiente línea.

Fuente: http://www.eyecanlearn.com/tracking/

2. Procesamiento léxico. Se trata del proceso clave de la lectura pues si una persona falla en el reconocimiento de las palabras no será capaz de leer con fluencia. Un factor muy importante para el reconocimiento de una palabra es su frecuencia, o sea, las palabras más fáciles y rápidas de reconocer son las que se utilizan comúnmente. Durante el reconocimiento de las palabras, las letras son sacadas de una pequeña área de la memoria visual a corto plazo, una a una. Por lo tanto, el tiempo de nombramiento aumenta en función del número de sílabas en una palabra, resultando más difícil identificar las palabras largas que las cortas $^{134}$. Para alcanzar el conocimiento que una persona posee de las palabras, es necesario observar la palabra escrita y compararla con una serie de representaciones almacenadas en el léxico mental, que es una especie de diccionario mental donde se encuentran representadas todas las palabras conocidas por el lector ${ }^{136}$. Una vez identificada la palabra, el siguiente paso es acceder a los significados que tienen asociados a ella en su memoria. Para ello, es necesario acudir a otro componente denominado sistema semántico.

3. Procesamiento sintáctico. A pesar de la importancia del procesamiento léxico en el desarrollo de la lectura, que una persona sea capaz de reconocer palabras no es garantía de que sea un buen lector. Las palabras aisladas no producen ninguna información nueva, sino que es como están relacionadas entre sí dentro de la oración y sus respectivos roles sintácticos, donde se encuentra el mensaje ${ }^{137}$. 
4. Procesamiento semántico. El reconocimiento de una palabra y la comprensión de su significado no son procesos simultáneos. Las representaciones semánticas de las palabras se encuentran almacenadas en un lugar de nuestra memoria diferente al léxico visual. Cuando se lee una palabra se accede al léxico visual para lograr reconocerla visualmente, sin embargo para lograr comprenderla es necesario acceder al almacén de los significados conocido por sistema semántico. Es el último proceso que consiste en extraer el significado de la oración e integrarlo con los conocimientos previos del lector. Cuando la información está integrada en la memoria, se puede decir que el proceso de comprensión ha terminado ${ }^{132}$.

\subsubsection{La velocidad lectora y la discapacidad visual}

La evaluación de la velocidad lectora (VL) es como un índice de fluidez de la lectura. Es decir, una lectura fluida significa una buena capacidad para identificar las palabras, contribuyendo a una mejor comprensión del texto ${ }^{138}$. Es una herramienta importante en los trastornos de la lectura y la utilización de textos estandarizados para ello es crucial.

Uno de los factores que influencia la VL es la edad. Al comparar la VL entre 100 voluntarios con visión normal de edades entre 18-35 años (25 para cada idioma: Inglés, Finlandés, Francés, y Alemán) y 100 voluntarios con visión normal de edades entre 60-85 años (25 por idioma), Hahn et al. ${ }^{139}$ encontraron que la VL en el grupo de jóvenes fue $20 \%$ más rápida que en el grupo de personas mayores. La medida de la VL en personas mayores (edad media de $64.5 \pm 7.2$ años) también fue estudiada por Altpeter et al. ${ }^{140}$, dónde utilizó dos tests estandarizados: el IReST y el Radner. La media de la VL encontrada utilizando el test de lectura Radner fue de $170 \pm 33 \mathrm{ppm}$. En un estudio similar pero con un grupo más joven (edad media de 64.5 \pm 7.7 años), Radner y Diendorfer ${ }^{141}$ encontraron velocidad media de lectura de $202 \pm 36 \mathrm{ppm}$. Así que, comparando los resultados de ambos estudios es posible afirmar que la lectura de las personas jóvenes es más rápida que la de las personas mayores.

El factor visual también es un aspecto de gran influencia en la VL y, para que una persona con BV pueda realizarla de manera eficiente es necesario: una AV de cerca suficiente para ver las letras, un CV con extensión aceptable para procesar las palabras y planear los movimientos oculares, adecuada SC para leer textos poco contrastados, buena adaptación a la luz y las funciones motrices del ojo apropiadas ${ }^{139}$. Con lo cual entre las limitaciones y dificultades que las personas con BV relatan está la capacidad para leer, considerada una función clave en la realización de actividades en la vida cotidiana.

Muchas de las personas con BV demandan a los servicios de RV mejorar la velocidad y la precisión de la lectura, a través del entrenamiento visual, la 
prescripción de ayudas ópticas y la instrucción adecuada para su utilización ${ }^{8,142}$. Existen estudios que investigaron la VL en personas mayores con BV, además de los efectos de un programa de RV sobre la misma. Al investigar la VL en personas mayores con una media de edad de 79 años y con degeneración macular relacionada a la edad (DMAE), Rubin y Feely ${ }^{143}$ encontraron una VL media de 54ppm y el programa de RV realizado no tuvo éxito. Sin embargo, otro estudio realizado con una población de características similares encontró una VL media de 83.3ppm ${ }^{144}$.

Las ayudas ópticas suelen proporcionar una mejora significativa en la VL de las personas con BV. Un estudio realizado con 530 personas mayores en diferentes estadios de la DMAE corrobora esa afirmación. Dicho estudio utilizó textos estandarizados para evaluar la VL en esa muestra antes y después de la prestación de ayudas ópticas de BV adecuadas. La media de VL fue de $20 \pm 33$ ppm antes y aumentó significativamente a $72 \pm 35$ ppm $(p<0.0001)$ después del suministro de las ayudas ópticas para todo el grupo ${ }^{13}$.

Las ayudas visuales para cerca tienen la función de aumentar el tamaño de la imagen del objeto sobre la retina, aportando una imagen ampliada del mismo. Existen diferentes tipos de lupas, como las lupas de mano (LM), lupas con soporte (LS), lupas de mano con iluminación (LMI), las lupas con soporte e iluminación (LSI), de bolsillo y las electrónicas (Figura 12) ${ }^{84}$.

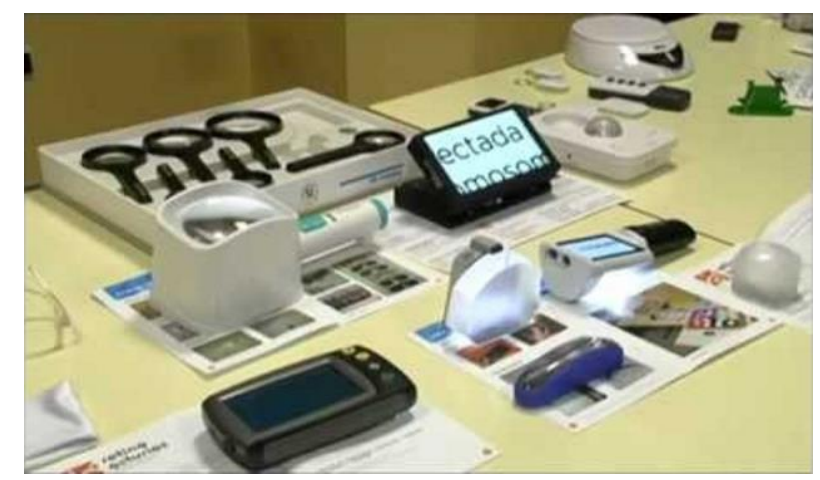

Figura 12: Tipos de lupas.

Fuente: http://img.youtube.com/vi/zjHqk_R7rl/0.jpg

Una cuestión a considerar en el uso de una ayuda de BV es el papel de los factores no visuales, como la postura corporal adoptada y los movimientos de la mano. Por ejemplo, utilizar una lupa de mano implica un esfuerzo físico considerando que el individuo tiene que utilizar las dos manos para navegar por las páginas de manera adecuada. Es decir, el usuario tiene que mover la lupa a la derecha a lo largo de las líneas del texto mientras mantiene la distancia lente-objeto. Al final de la línea, es necesario un barrido de retorno a la izquierda para encontrar el principio de la línea siguiente ${ }^{10,145}$. 
Algunas personas tienen dificultades para realizar el movimiento de retorno al principio de la línea siguiente con la lupa, con lo cual saltan y/o repiten líneas perdiéndose en el texto y dificultando su comprensión ${ }^{10,146}$.

En lo que se refiere a la relación existente entre la $V L$ y la postura, pocos son los estudios sobre el tema. Firth et al. ${ }^{24}$ investigaron la relación entre la inclinación de la cabeza, del cuerpo y del texto en la VL de adultos jóvenes sin DV. Sus resultados indicaron que la inclinación del texto, de la cabeza o del cuerpo, afecta a la lectura. Datos estos confirmados por el estudio de Perrin et al. ${ }^{147}$ al verificar el efecto de la inclinación lateral de la cabeza en la lectura. Ambos estudios añaden que si dicha inclinación es pequeña, los sujetos son capaces de adaptarse a ella, no afectando el desempeño en la lectura.

El equilibrio corporal también es un factor que debería ser tenido en cuenta a la hora de elaborar un programa de $\mathrm{RV}$, considerando que el mismo se encuentra disminuido en las personas con DV. Sin embargo, su déficit no produce alteración en el desempeño de la lectura ${ }^{148}$.

Tal información fue confirmada por Radvay et al. ${ }^{149}$, que investigó el impacto de un programa de intervención para mejorar el equilibrio sobre la VL en personas mayores con DMAE. Aunque el programa de rehabilitación realizado logró mejorar el equilibrio corporal, la VL no mejoró de manera estadísticamente significativa en comparación con el grupo control.

A pesar de los hallazgos descritos anteriormente, hasta el día de hoy no hemos encontrado estudios que hayan examinado la relación entre las alteraciones posturales y del movimiento y la VL en personas mayores con BV. Quizá por eso la mayoría de los programas de RV están centrados en solucionar los problemas puramente visuales sin sopesar tales alteraciones.

\subsection{Destreza manual}

La mano es un complejo órgano de la extremidad superior que tiene muchas responsabilidades en la actividad diaria y que posee tanto habilidades motoras como discriminativas sensitivas importantes. Es también utilizada como un órgano sensorial para proporcionar información sobre el medio ambiente. Su funcionamiento depende de la relación entre distintos elementos como los huesos, ligamentos, tendones y músculos. Para coger algún objeto, la mano tiene que aptarse a su forma y la manera de cogerlo o sujetarlo puede ser de dos tipos: garra (utilizando toda la mano) y pinza (que se realiza con algunos dedos) $)^{42}$.

La sensibilidad y la fuerza de la mano se combinan para producir las habilidades manipulativas o destrezas que facilitan los movimientos. Según Backman et al. ${ }^{150}$, la destreza se define como "los movimientos finos, voluntarios que se utilizan para manipular objetos pequeños durante una tarea específica". Las evaluaciones utilizadas en la destreza manual son capaces de 
cuantificar y predecir tanto la capacidad y la discapacidad como la calidad del movimiento de una persona, como el habilidad de la mano con objetos y herramientas relacionadas con el autocuidado, trabajo o actividades de ocio ${ }^{151}$.

La evaluación de la destreza manual es fundamental por ser un elemento central de la función de la mano, ofreciendo informaciones acerca de la independencia en la realización de las actividades de vida diaria en distintas poblaciones. En la actualidad, están disponibles muchas evaluaciones de destreza diferentes y la elección de una herramienta de evaluación adecuada muchas veces se basa en distintos elementos como: el costo financiero, el tiempo de aplicación, la disponibilidad, la familiaridad, la practicidad y la aplicabilidad a la población estudiada ${ }^{152}$.

La destreza manual de una persona puede verse afectada debido a diversos factores y uno de ellos es la edad. Las personas con edad avanzada pueden presentar deterioro sensorial y/o motor afectando la coordinación motora, la destreza manual, la fuerza muscular y la sensibilidad de las manos, requisitos importantes para el desempeño adecuado de las tareas manuales. Amirjani et al. ${ }^{153}$ evaluaron la destreza manual en grupos de diferentes edades, confirmando que el rendimiento en la prueba utilizada difirió de manera estadísticamente significativa entre los jóvenes, de mediana edad, y los sujetos de mayor edad.

En los adultos mayores, las habilidades motoras gruesas (por ejemplo, para alcanzar y agarrar), así como las habilidades motrices finas (por ejemplo, para la manipulación) son más lentas y menos precisas que en los adultos jóvenes normales y, se añadimos a esto los problemas visuales resultantes de la edad avanzada, las consecuencias sobre la destreza manual son todavía peores. De hecho, hay estudios que indican que la disminución de la velocidad en los movimientos 0 en tareas repetitivas están asociadas a cambios visuales relacionados con la edad ${ }^{154,155}$. En un estudio para investigar la relación entre la percepción visual y la destreza manual en personas mayores, se encontró que tanto el deterioro de la percepción visual como el deterioro cognitivo pueden influir en la destreza manual en los adultos mayores, sugiriendo que la rehabilitación de la función de la extremidad superior, junto con el entrenamiento de la percepción visual y cognitiva podría beneficiar a esta población ${ }^{156}$.

Las personas mayores son el grupo poblacional más prevalente en DV y debido a la disminución de la coordinación motora, común en la edad avanzada, dichas personas presentan una destreza manual reducida ${ }^{145,157}$.

A pesar de una considerable cantidad de literatura sobre la evaluación de la destreza manual, se han realizado pocos estudios sobre sus implicaciones funcionales y la relación entre las habilidades motoras y la VL en personas mayores con BV. 
La primera referencia encontrada en la literatura evaluando el efecto de la destreza manual en la lectura con una lupa, aparece en la revista Investigative Ophthalmology \& Visual Science en 2007 en un artículo titulado The influence of manual dexterity on reading speed with a hand-held magnifier, firmado por Dickinson ${ }^{139}$. Sus resultados indicaron una fuerte relación entre la destreza manual y la velocidad lectora con lupa en personas mayores, sin embargo, los participantes de este estudio no presentaban BV.

La evaluación de la destreza manual puede ayudar a identificar a las personas que tienen dificultades para utilizar las ayudas ópticas necesarias, lo que puede facilitar la elección del tipo de ayuda a prescribir, evitando el rechazo de los pacientes a las mismas y favoreciendo el proceso de RV.

\subsection{Repercusiones psicológicas de la Discapacidad Visual}

La disminución severa de la visión es una situación adversa que puede interferir significativamente con la capacidad funcional para realizar tareas de manera independiente. Está asociada con un aumento de la insatisfacción con la vida y la aparición de los síntomas depresivos en un amplio porcentaje de personas mayores que afrontan un proceso de pérdida de visión. La depresión y el ajuste a la pérdida de visión afecta directamente la autopercepción del individuo independientemente de la severidad de la pérdida visual, reduciendo su calidad de vida ${ }^{158,159}$. Diferentes estudios apuntan que las personas mayores con déficit visual sufren una angustia emocional mayor que las personas mayores sin déficit visual, principalmente cuando dicha condición es reciente ${ }^{160,161}$. Y, que existe una posible relación de reciprocidad entre la depresión y la DV, es decir, la discapacidad puede llevar a la depresión y la depresión influye en la discapacidad.

Estos datos pueden explicarse porque el déficit visual conduce a la restricción de algunas actividades de la vida diaria y aficiones tales como la lectura, incapacidad o limitación con respecto a la conducción, dificultad para realizar tareas relacionadas con el trabajo o las actividades de ocio, como coser 0 cocinar $^{162}$. Los impedimentos en la realización de algunas de estas tareas o de actividades de la vida diaria implican una pérdida importante de la independencia del individuo, teniendo en cuenta que él tiene que dejar de hacerlas o pedir que alguien le ayude. Esto también influye en su estado emocional ${ }^{163,164}$.

Las relaciones sociales y familiares también se ven afectadas por la pérdida visual. Dicha población es más propensa a sentirse sola y carente de apoyo social, tendiendo al aislamiento y consecuentemente a disminución de las relaciones sociales. El hecho de no ser capaz de desempeñar adecuadamente las actividades laborales también es un factor importante, teniendo en cuenta 
que puede llevar a un sentimiento de inutilidad, así como a consecuencias económicas considerables ${ }^{165,166}$.

La edad a la que surge la DV es un factor importante, teniendo en cuenta que las necesidades son diferentes para cada franja etaria. Las personas mayores tienen que afrontar además de las dificultades por la pérdida visual, los cambios en la morfología lumbar, los cuales conducen a la fragilidad en los tejidos conectivos y la reducción de la fuerza muscular. Tales alteraciones contribuyen al deterioro de la alineación de la columna y la postura en dicha población.

Diversas investigaciones demuestran que las personas mayores son más dependientes de la visión en la ejecución de determinadas tareas tales como: estabilidad postural, marcha y postura corporal. Con lo cual, la existencia de una DV en un anciano supone una mayor dificultad para la realización de las mismas, lo que aumenta el riesgo de caídas y el miedo a caerse ${ }^{67,69,127,149,167-}$ 172 .

Ambos aspectos están muy relacionados entre sí y tienen gran influencia sobre el proceso de envejecimiento. El miedo de caerse se define como una preocupación permanente por la caída que puede llevar a una persona a evitar las actividades que él/ella sigue siendo capaz de realizar ${ }^{173}$. La preocupación por caerse es común en la vejez y puede llevar a diferentes consecuencias como deterioro funcional, la restricción de la participación social ${ }^{174}$, disminución de la calidad de vida, mayor riesgo de caídas y la institucionalización ${ }^{175}$. Se estima que el $30-55 \%$ de las personas mayores reconocen tener miedo a caerse, lo que le da el reconocimiento como un problema de salud entre los adultos mayores. $Y$ cuando este miedo resulta en evitación de actividades y la reducción de la aptitud física, es un factor de riesgo para las caídas futuras y mortalidad asociada ${ }^{176}$.

Hay una gran variación en la prevalencia del miedo a caerse en las personas mayores así como múltiples factores asociados. El conocimiento de sus factores de riesgo es muy importante para el desarrollo de estrategias multidimensionales de afrontamiento. El dolor es considerado un factor de riesgo para el miedo a caerse además de ser un síntoma común en los adultos mayores, ya que los cambios posturales en la curvatura lumbar, como la disminución (rectificación lumbar) o el aumento (hiperlordosis) contribuyen al dolor en la espalda baja ${ }^{177}$.

En un reciente estudio sobre la prevalencia y el impacto del dolor en 7601 personas mayores de 65 años, el dolor afligió más de la mitad de la población, tres cuartos de ellos tenían dolor en múltiples regiones del cuerpo, y se asoció a disminución de la fuerza manual, de la velocidad de la marcha, así como a la mala función de la extremidad inferior ${ }^{178}$. El dolor crónico podría predisponer a 
las personas mayores a caer debido al miedo al movimiento ${ }^{179}$, a la falta de actividad física ${ }^{180}$, la inestabilidad postural y alteración de la marcha ${ }^{181}$.

Percibir los déficits funcionales y psicológicos que experimentan las personas con BV es un problema difícil. Hay tres diferentes dimensiones a cerca de la BV y la VL que deben ser llevadas en cuenta a la hora de estudiar esta población: el papel de las variables visuales, de las funcionales y el papel de las variables psicológicas. 

3. HIPÓTESIS DE TRABAJO Y OBJETIVOS 



\subsection{Hipótesis de trabajo}

Las personas mayores con BV presentan más alteraciones posturales, del equilibrio y de destreza manual que las personas mayores sin BV, afectando negativamente a su velocidad lectora. Además, elevados niveles de sintomatología depresiva y/o ansiosa empeorarán más dichas dificultades motrices y lectoras.

\subsection{Objetivos}

- General

Analizar y comparar la influencia de la postura corporal, equilibrio y destreza manual en la velocidad lectora de personas mayores con y sin baja visión.

- Secundarios

I. Comparar las alteraciones posturales, de equilibrio, visuales y la destreza manual en ambos grupos participantes, con BV y sin BV.

II. Examinar la fiabilidad y la validez intraobservador del sensor que mide la postura corporal utilizado en este estudio (LUMOback).

III. Evaluar la fiabilidad y la validez intraobservador de la demarcación de los puntos anatómicos utilizados para la evaluación postural.

IV. Comprobar si hay diferencias en la VL de las personas mayores con y sin BV en función de sus parámetros posturales, visuales, de equilibrio corporal y de destreza manual y determinar los factores posturales predictivos en el aumento de la dificultad lectora.

V. Analizar las posibles relaciones entre el nivel de sintomatología depresiva y las dificultades posturales, motrices, la velocidad lectora, el miedo a caerse y el dolor y comprobar si hay diferencias en los dos grupos de participantes en esta sintomatología depresiva, el miedo a caerse y el dolor. 

4. METODOLOGÍA DE LA INVESTIGACIÓN 

El estudio que presentamos se realizó considerando los principios éticos para las investigaciones médicas en seres humanos de la Declaración de Helsinki de la Asociación Médica Mundial de 1964 y sus posteriores revisiones. Y fue aprobado por el Comité Ético de la Facultad de Medicina, de la Universidad de Valladolid (Anexo I). Todos los participantes firmaron el debido consentimiento informado (Anexo II) antes comenzar el estudio.

\subsection{Participantes}

Para la selección de la muestra se establecieron los criterios de inclusión y exclusión que se detallan en las Tablas 2 y 3 . La muestra se dividió en dos grupos, grupo control (sin BV) y grupo caso (con BV). Se clasificó a los participantes en dos grupos (con BV y sin BV) dependiendo de la AV presentada. Dentro del grupo con $B V$ se incluyeron a las personas con $A V \geq 0.4$ $y \leq 1.3$ en logMAR. El grupo muestral fue mayor en el de participantes sin BV $(n=49)$.

Tabla 2: Criterios de inclusión de los participantes.

\begin{tabular}{cc}
\hline Grupo Caso & Grupo Control \\
\hline Ser mayor de 60 años. & Ser mayor de 60 años. \\
$\begin{array}{c}\text { Tener una agudeza visual } \leq 0,4 \text { en el } \\
\text { mejor ojo, en notación decimal. } \\
\text { Y/o padecer un defecto de campo } \\
\text { visual central inferior a 20․ }\end{array}$ & $\begin{array}{c}\text { Tener agudeza visual }>0,4 \text { en } \\
\text { el peor ojo, en notación } \\
\text { decimal. }\end{array}$ \\
\multicolumn{2}{c}{ No padecer un defecto de } \\
Campo visual.
\end{tabular}

La procedencia y los detalles del protocolo de captación de esta muestra se explicitan en el apartado de procedimiento. 
La edad media de los sujetos que participaron en el estudio fue de 72.25 (6) años. El $38.75 \%$ de los participantes $(n=31)$ fueron varones y el $61.25 \%$ mujeres $(n=49)$. El 61.25\% $(n=49)$ estaban casados, $11.25 \%(n=9)$ solteros, $2.5 \%(n=2)$ separados y $25 \%(n=20)$ viudos. No se encontraron diferencias estadísticamente significativas en la edad media para los dos grupos, con y sin BV $(p=0.98)$.

En lo que se refiere al índice de masa corporal (IMC), 33.75\% ( $n=27)$ fueron considerados normales $\left(18.50 \mathrm{Kg} \cdot \mathrm{m}^{2}-24.99 \mathrm{Kg} \cdot \mathrm{m}^{2}\right), 41.25 \% \quad(\mathrm{n}=33)$ tenían sobrepeso $\left(25 \mathrm{Kg} \cdot \mathrm{m}^{2}-29.99 \mathrm{Kg} \cdot \mathrm{m}^{2}\right)$ y $25 \%(\mathrm{n}=20)$ eran obesos $\left(\geq 30 \mathrm{Kg} \cdot \mathrm{m}^{2}\right)$. El miembro dominante derecho fue encontrado en casi la totalidad de la muestra, $98.75 \%(n=79)$. No se encontraron diferencias estadísticamente significativas en el IMC entre los dos grupos, con y $\sin B V(p=0.3)$.

Por lo tanto, ambos grupos estaban equiparados tanto en edad como en su índice de masa corporal.

\subsection{Diseño}

En nuestra investigación se utilizó un diseño cuasi-experimental de casocontrol. Es decir, los sujetos fueron seleccionados en función de padecer una determinada enfermedad, en este caso la baja visión (grupo caso) o no padecerla (grupo control).

El diseño que llevamos a cabo se concretó en dos momentos distintos: 1) Visita inicial, que se realizó a los participantes de manera individual independiente del grupo al que pertenecía, donde se llevó a cabo la evaluación de las todas las variables (Tabla 4) y tuvo una duración aproximada de 90 minutos; 2) Visita final, que se aplicó a los mismos participantes de la visita inicial, donde se realizó la evaluación de dos variables físicas (ángulos posturales en bipedestación y en sedestación) y duró en media 30 minutos.

Tabla 4: Variables evaluadas.

\begin{tabular}{|c|c|c|c|}
\hline \multicolumn{4}{|c|}{ Variables Físicas } \\
\hline $\begin{array}{c}\text { Velocidad lectora } \\
\text { (variable } \\
\text { dependiente) }\end{array}$ & $\begin{array}{c}\text { Ángulos posturales } \\
\text { en bipedestación } \\
\text { (variable } \\
\text { independiente) }\end{array}$ & $\begin{array}{c}\text { Equilibrio } \\
\text { (variable } \\
\text { independiente) }\end{array}$ & $\begin{array}{c}\text { Destreza manual } \\
\text { (variable } \\
\text { independiente) }\end{array}$ \\
\hline \multicolumn{4}{|c|}{ Variables Visuales } \\
\hline \multicolumn{4}{|c|}{ Sensibilidad al contraste (variable de confusión) } \\
\hline \multicolumn{4}{|c|}{ Variables Psicológicas } \\
\hline $\begin{array}{r}\text { Deterioro cognitiv } \\
\text { exclusi }\end{array}$ & $\begin{array}{l}\text { Depresión (variable } \\
\text { dependiente) }\end{array}$ & $\begin{array}{l}\text { Miedo a caerse } \\
\text { (variable } \\
\text { independiente) }\end{array}$ & $\begin{array}{l}\text { Dolor (variable } \\
\text { independiente) }\end{array}$ \\
\hline
\end{tabular}




\subsubsection{Descripción de las variables}

\subsubsection{Variables Físicas}

- Velocidad lectora: evaluada con el test IReST y representada por el número de palabras leídas correctamente por minuto ${ }^{182}$.

- Ángulos posturales en sedestación: evaluados con la fotogrametría a través del software SAPO.

- Ángulos posturales en bipedestación: evaluados con la fotogrametría a través del software SAPO ${ }^{109}$.

- Equilibrio corporal: evaluado con el test Timed Up and Go (TUG) ${ }^{183}$.

- Destreza manual: evaluada con el Pick Up test. Se evaluó ambas manos con los ojos abiertos y cerrados ${ }^{184}$.

\subsubsection{Variables Visuales}

- Agudeza visual (AV): evaluada con la tabla Early Treatment Diabetic Retinopathy Study (ETDRS) y transformada en logMAR. Se recogió la AV en ambos ojos y se tomó como representante la AV media de los dos ojos.

- Sensibilidad al contraste (SC): evaluada con el test de Pelli-Robson recogida en tres variables: ojo derecho, ojo izquierdo y binocular. Teniendo en cuenta que estas tres variables estaban muy relacionadas entre sí (este dato fue verificado utilizando el coeficiente de correlación de Spearman), se eligió la variable binocular como representante.

\subsubsection{Variables Psicológicas}

- Deterioro cognitivo: se evaluó la existencia y nivel del deterioro cognitivo a través del Examen Cognoscitivo Minimental ${ }^{185}$.

- Depresión: se evaluó la presencia de sintomatología depresiva con la Escala de Depresión Geriátrica (GDS).

- Miedo a caerse: se evaluó el nivel de preocupación por caerse con el Falls Efficacy Scale-International (FES-I).

- Dolor: se evaluó el dolor percibido con la Escala Visual Analógica (EVA) ${ }^{186}$. 


\subsubsection{Terapeuta}

Todos los participantes fueron evaluados según los criterios de exclusión y de inclusión por la misma profesional con formación adecuada para realizar todas las pruebas.

\subsection{Materiales e instrumentos}

Los materiales utilizados fueron: una cámara fotográfica digital Nikon Coolpix S2700, un trípode Starblitz TS-110, bolas de poliestireno de $15 \mathrm{~mm}$, cinta adhesiva doble cara, alfombra negra de goma EVA $70 \times 74 \mathrm{~cm}$, tiza blanco, metro, dos plomadas, cronómetro, caja con 12 objetos para el test de destreza manual, software para el análisis postural (SAPO), iPhone, LUMOback y aplicativo del LUMOback.

Los instrumentos utilizados para cada prueba se describen a continuación y un ejemplar de cada uno de ellos se recoge en el Anexo III.

\subsubsection{Evaluación física}

- New International Reading Speed Texts - IReST: es un test que evalúa el rendimiento de lectura y está traducido en 17 idiomas. La versión en español fue creada por Trauzettel-Klosinski y Dietz ${ }^{182}$ en 2012 y consiste en marcar el tiempo que le lleva a una persona leer un texto específico registrando las palabras omitidas o leídas incorrectamente. El cálculo de la velocidad de lectura se hace a través de la fórmula: 60 x número de palabras leídas correctamente (omitidas o leídas incorrectamente número de palabras del texto)/tiempo de lectura en segundos.

Para evaluar la postura corporal, se utilizaron dos herramientas distintas:

- El Software de Evaluación Postural (SAPO), desarrollado para la evaluación de la postura en imágenes digitalizadas permitiendo la medición de distancias y ángulos. Se creó en 2010 por Ferreira et al. ${ }^{187}$, y ha sido validado en muchos otros estudios ${ }^{109,111,188}$.

- El sensor postural LUMOback (Figura 13), a través del registro de las medidas de la flexión lumbar en la postura sentada. Es un aparato dotado de un sensor que utiliza tres insumos para determinar la buena postura: el ángulo de la espalda baja y la pelvis; un acelerómetro interno para determinar el movimiento; y los algoritmos de movimiento que juntamente con el acelerómetro determinan la posición. Al calibrar el sensor con una buena postura, sus ángulos son utilizados como base para medir los cambios en la inclinación lumbar. Su fiabilidad y validez han sido evaluadas en este estudio. 


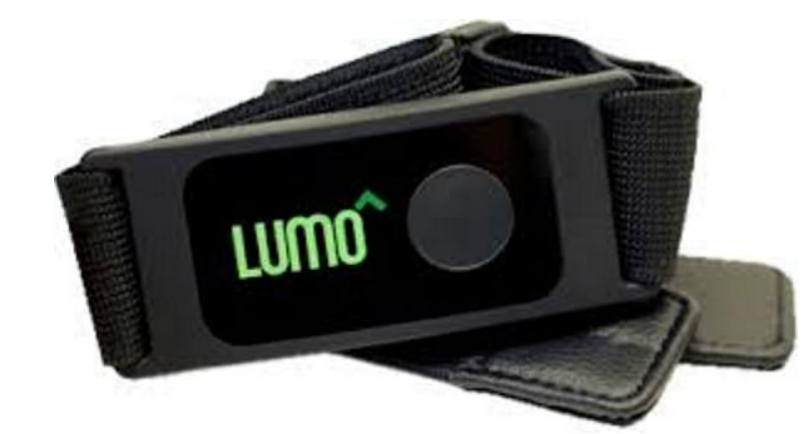

Figura 13: LUMOback

Fuente: http://www.LUMObackbodytech.com/LUMObackback/

- Timed Up and Go Test (TUG): esta prueba es una versión modificada cronometrada del "Get-Up and Go" Test ${ }^{189}$. Fue desarrollada en 1991 por Podsiadlo y Richardson y consiste en marcar el tiempo, en segundos, que una persona lleva para levantarse de una silla, caminar una distancia de 3 metros, girar, caminar de regreso a la silla y sentarse ${ }^{183}$. Ha sido extensamente usada para examinar el equilibrio, la velocidad de la marcha y la capacidad funcional en personas mayores ${ }^{190}$.

- Pick Up Test: es un test breve y objetivo de la destreza manual que involucra la recogida, explotación, la manipulación y la identificación de objetos pequeños. La prueba fue desarrollada por Moberg en $1958^{184}$ y consiste en la recogida de 12 objetos pequeños (por ejemplo, un clip de papel, monedas) y colocarlos en un recipiente pequeño. Cada mano se evalúa por separado y los mismos procedimientos se repiten con los ojos cerrados.

\subsubsection{Evaluación visual}

En primer lugar se realizaron las pruebas oftalmológicas para la evaluación de la función visual. La mejor AV de lejos corregida se midió con el ETDRS y posteriormente se transformó a logMAR y la SC se evaluó con el test de PelliRobson en ternas. Todas las pruebas visuales han sido realizadas por un óptico-optometrista del Instituto Universitario de Oftalmobiología Aplicada de la Universidad de Valladolid (IOBA).

\subsubsection{Evaluación psicológica}

Después se realizaron las pruebas psicológicas que se aplicaron mediante entrevista, las cuales se describen a continuación.

- Examen Cognoscitivo Minimental de Folstein y colaboradores (traducido y adaptado al castellano por Lobo y otros). Este instrumento se utilizó en la evaluación inicial con el fin de comprobar el cumplimiento, por parte de los participantes, del criterio de inclusión-exclusión de nuestra investigación. 
Se trata de una prueba para detectar el deterioro cognitivo y vigilar su progresión. Fue diseñada por Folstein y McHungen en $1975^{191}$ y hemos utilizado la versión de Lobo et al. de $1980^{185}$ que está adaptada y validada en español. Sus ítems exploran 5 áreas cognitivas: Orientación, Fijación, Concentración y Cálculo, Memoria y Lenguaje. El resultado es obtenido a través de la suma directa de los aciertos logrados en la prueba pudiendo llegar a un valor máximo de $30 \mathrm{y}$, cuanto menor la puntuación, mayor el deterioro cognitivo.

Los ítems "Dibuje dos pentágonos intersectados y pida al sujeto que los copie tal cual" o "Escriba una frase con sujeto y predicado" se excluyeron cuando eran imposibles de cumplimentar por imposibilidad física (ceguera) o por analfabetismo. En estos casos, se calculó la corrección correspondiente a través de una regla de tres. Por ejemplo, si la persona mayor con BV no pudo acceder a 4 de los 30 puntos posibles, la puntuación final se calculó sobre los 26 puntos posibles. Es decir, supongamos que la puntuación total había sido 20, aplicando la corrección obtenemos una puntuación $(20 \times 30 / 26)$ de 23.07 (redondeamos al número entero más próximo, en este caso 23$)^{192}$.

- Escala de Depresión Geriátrica de Yesavage - GDS: es un instrumento para medir la sintomatología depresiva en adultos mayores, que ha sido ampliamente utilizado en el estudio de la depresión en personas mayores tanto en el ámbito comunitario como en el clínico. Yesavage et al. en $1986^{193}$, diseño una versión corta de la escala que consistió de 15 preguntas. Una mayor puntuación indica mayor nivel de depresión. Para este estudio se utilizó la versión corta traducida al español, adaptada y validada por Martínez et al. ${ }^{194}$.

- Falls Efficacy Scale-International (FES-I): es una versión modificada del Falls Efficacy Scale y evalúa el nivel de preocupación por caerse durante las actividades físicas y sociales dentro y fuera del hogar de los adultos mayores con o sin antecedentes de miedo a caerse. Fue desarrollada por Yardley et al. ${ }^{195}$ en 2005 centrándose en la prevención de caídas y la psicología de la caída. La FES-I fue utilizada en diferentes muestras en diferentes países y traducida en varios idiomas. Se utilizó la versión adaptada y validada en español de Lomas-Vega ${ }^{196}$.

- Escala Visual Analógica (EVA): es una escala simple y sólida, útil para evaluar el dolor percibido en diferentes zonas del cuerpo, en el mismo paciente y en diferentes ocasiones. Dicha escala consiste en una línea horizontal de $100 \mathrm{~mm}$ de longitud, presentando en cada extremo "No hay dolor" y "peor dolor imaginable". A los participantes se les pidió hacer una marca en la línea que mejor representara el nivel de intensidad del dolor que estaban experimentando en el momento de la evaluación. La escala ha sido reproducida en un tamaño que pudiera ser vista por los 
participantes con BV. La versión utilizada en este estudio fue publicada por primera vez en la década de 1920 por Hayes y Patterson ${ }^{186}$ y es la base de toda las variaciones publicadas posteriormente ${ }^{197}$.

\subsection{Procedimiento}

Se evaluaron las variables físicas (VL, postura corporal en sedestación y en bipedestación, equilibrio y destreza manual), las visuales (AV y SC), así como las psicológicas (deterioro cognitivo, depresión, miedo a caerse y dolor) en la visita inicial. Participaron de dichas pruebas 80 personas mayores con y sin BV.

El procedimiento para evaluar la fiabilidad intraobservador de las medidas posturales utilizando el protocolo SAPO se llevó a cabo en dos días distintos. Teniendo en cuenta que el segundo día de evaluación se realizó con un intervalo de dos meses, solamente 40 sujetos de los 80 participantes iniciales accedieron a repetir dicho procedimiento.

Para determinar la fiabilidad y validez del sensor postural LUMOback, se evaluaron 64 personas de las 80 que aceptaron participar del estudio. Se repitió la misma medición cinco veces en el grupo control, tres en la primera visita y otras dos en la segunda, y dos veces en el grupo caso, ambas en la misma visita.

\subsubsection{Captación de los participantes}

Los participantes procedían de tres lugares diferentes: de las consultas de oftalmología del IOBA, de la Asociación Castellano-Leonesa de Afectados de Retinosis Pigmentaria (ACLARP) y del Centro de Personas Mayores Zona Sur del Ayuntamiento de Valladolid.

En el caso de los pacientes de las consultas de oftalmología del IOBA, se contó con la colaboración de los oftalmólogos de las mismas que, teniendo en cuenta los criterios de inclusión y exclusión, ofrecieron en primer lugar a los pacientes la posibilidad de participar en la investigación. Se les informó de los objetivos, beneficios y procedimientos de la investigación, se les tomó nota de su nombre, domicilio y teléfono y se les citó para una entrevista individual. En el caso de la ACLARP, se mantuvo una reunión con la directiva y se presentó el estudio en la asamblea general de socios. Se tomó nota de aquellos socios que manifestaron su interés en participar citándoles para una entrevista individual. En el caso del Centro de Personas Mayores, se mantuvo una reunión con la directiva y se presentó el estudio en una reunión abierta a todos los interesados que frecuentaban dicho Centro. Se tomó nota de aquellos que manifestaron su interés en participar citándoles para una entrevista individual.

Se invitó inicialmente 174 personas que cumplían los criterios de inclusión a participar de esta investigación, de los que 80 se mostraron interesados 
después de la sesión informativa. Posteriormente, a todos ellos e individualmente se les leyó y pidió el consentimiento informado para su participación en la investigación.

Empezado el proceso de evaluación, después de realizar casi todas las pruebas 16 personas se negaron a cumplir la evaluación postural en sedestación. Las razones esgrimidas fueron que no les apetecía leer o que estaban demasiado cansadas, ya que dicha evaluación era la última realizada.

\subsubsection{Recogida de los datos}

Todos los sujetos de ambos grupos han realizado las mismas pruebas, siguiendo el mismo protocolo y el mismo orden de aplicación (ver Figura 14).

La evaluación postural y la velocidad lectora se realizaron al mismo tiempo puesto que ambas se midieron mientras el participante leía un texto. 


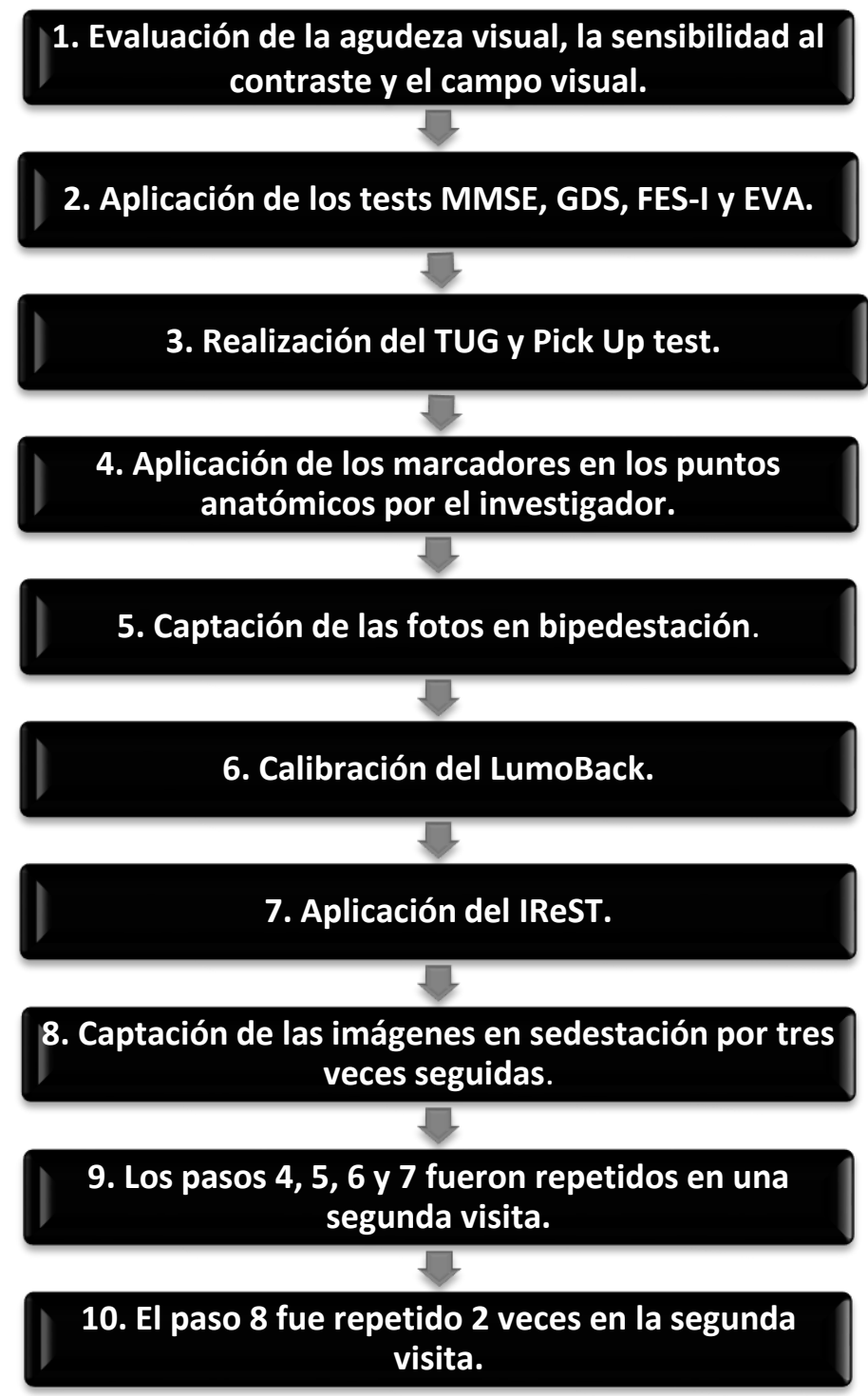

Figura 14: Procedimiento de recogida de datos para cada sujeto.

\subsubsection{Preparación y posicionamiento de los participantes}

Se evaluaron a todos los participantes de forma individual. En primer lugar se realizaron las pruebas visuales para la evaluación de la función visual. La mejor AV de lejos corregida se midió con el ETDRS y posteriormente se transformó a logMAR y la SC con el test de Pelli-Robson en ternas.

Después se realizaron las pruebas psicológicas que se aplicaron mediante entrevista, en la que el evaluador realizó las preguntas de cada test o cuestionario y dio las posibles alternativas de respuesta. Así mismo, se explicaron las dudas que los pacientes pudieran tener ante las diferentes preguntas. Este formato facilitó la ejecución de las mismas, dada la dificultad que muchos de estos pacientes tenían para poder leer y escribir.

La primera prueba física aplicada fue el Pick Up test. Sentado en una silla el participante tenía que colocar 12 ítems de diferentes tamaños en una caja con 
cada mano por separado mientras se marcaba el tiempo. En seguida se repitió el mismo procedimiento con los ojos cerrados. La siguiente prueba fue la de equilibrio, donde el participante tenía que levantarse de una silla, caminar por 3 metros, darse la vuelta, volver a silla y sentarse. Para la evaluación postural, al participante se le pidió que estuviera en ropa interior para que fuera posible la colocación de los marcadores en algunos puntos anatómicos, con el fin de identificar los puntos de referencia externos en las fotografías. En algunos puntos como en la $7^{\underline{a}}$ vértebra cervical $(\mathrm{C} 7)$ y en la $12^{\underline{a}}$ vértebra torácica $(\mathrm{T} 12)$ se colocaron dos marcadores superpuestos para permitir una mejor visibilidad por la cámara en la vista lateral. Se pidió al participante que se pusiese de pié sobre una alfombra y que adoptara su postura habitual. Se hizo una foto en vista lateral, en el lado dominante del participante. La cámara digital estaba posicionada perpendicularmente al participante, en un trípode con altura de 160 $\mathrm{cm}$ y a una distancia de $240 \mathrm{~cm}$.

El siguiente paso fue calibrar el LUMOback. Para ello, se pidió al participante que se sentara ligeramente hacia adelante, que pusiera las rodillas a la altura de las caderas, girase la pelvis hacia adelante y levantara el pecho. Se le pidió que mantuvieran esta posición alrededor de 30 segundos. Después, sentado en un taburete (ajustado a la altura de su línea poplítea), el participante leyó el texto específico utilizado para la evaluación de la VL mientras se hacían las fotografías. La cámara digital estaba posicionada perpendicularmente al lado dominante del participante, en un trípode con altura de $80 \mathrm{~cm}$ y a una distancia de $250 \mathrm{~cm}$. Para tornar la lectura más cómoda y fácil, se utilizaron un atril y un flexo de luz blanca y fría tales como en la consulta de RV en el IOBA.

\subsubsection{Puntos y ángulos medidos}

Los puntos óseos donde se colocaron los marcadores fueron: el canto externo del ojo, el trago, las apófisis espinosas C7 y T12, la espina ilíaca anterosuperior y el trocánter mayor. Se calcularon ocho ángulos posturales a partir de las imágenes digitales: flexión de la cabeza, flexión del cuello, ángulo cráneocervical, ángulo cérvico-torácico, flexión torácica, ángulo del tronco, ángulo lumbar e inclinación pélvica (Tabla 5). La elección de los puntos anatómicos y de los ángulos medidos se basa en otros estudios que han utilizado ángulos idénticos o similares $21,60,113,198,199$. 
Tabla 5: Puntos anatómicos y ángulos medidos.

ANGULOS

DESCRIPCION

DIAGRAMA

1. Flexión de la cabeza (FC)

Ángulo formado por encima de la intersección entre la

línea que une el canto lateral del ojo (A) al trago (B) y la

línea vertical que pasa por el trago $(B)$

Ángulo formado por encima

2. Flexión del cuello (FCu)

de la intersección entre la línea que une el trago (A) a C7 (B) y la línea vertical (C).

Ángulo anterior formado en la intersección entre la línea

\section{3. Ángulo cráneo-cervical} (ACC) que une el canto del ojo (A) al trago (B) y la línea que une el trago (B) al C7 (C).

Ángulo anterior formado en la intersección entre la línea

\section{4. Ángulo cérvico-torácico} (ACT)

que une el trago $(A)$ a $C 7(B)$

y la línea que une $C 7$ (B) a T12 (C).
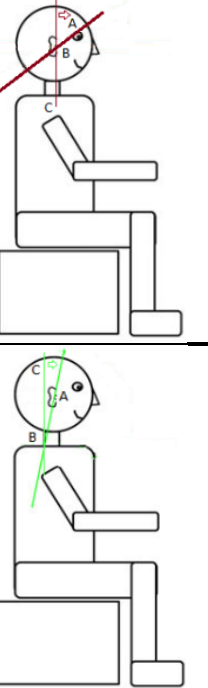

5. Flexión torácica (FT)

Ángulo formado a partir de la intersección entre la línea que une C7 (A) a T12 (B) y la línea vertical $(C)$.

Ángulo posterior formado entre la línea que une $C 7(A)$

6. Ángulo del tronco (AT) a T12 (B) y la línea que une T12 (B) al trocánter mayor del fémur (C).

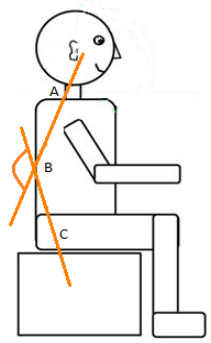




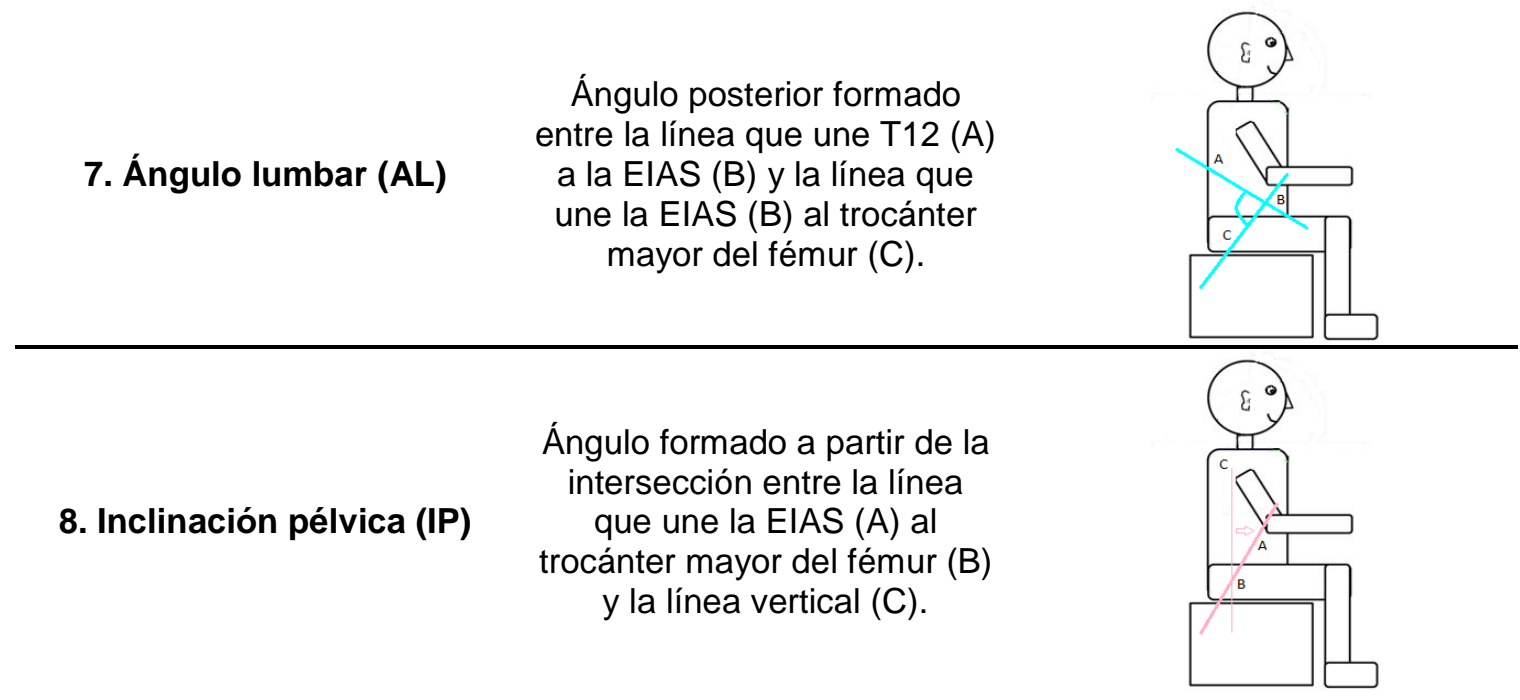

\subsection{Análisis estadístico}

Todo el análisis de los datos de nuestro estudio se llevó a cabo utilizando el programa informático R Statistical Software, versión 3.1.3200. El proceso de análisis se dividió en 4 fases, como muestra la Figura 15.

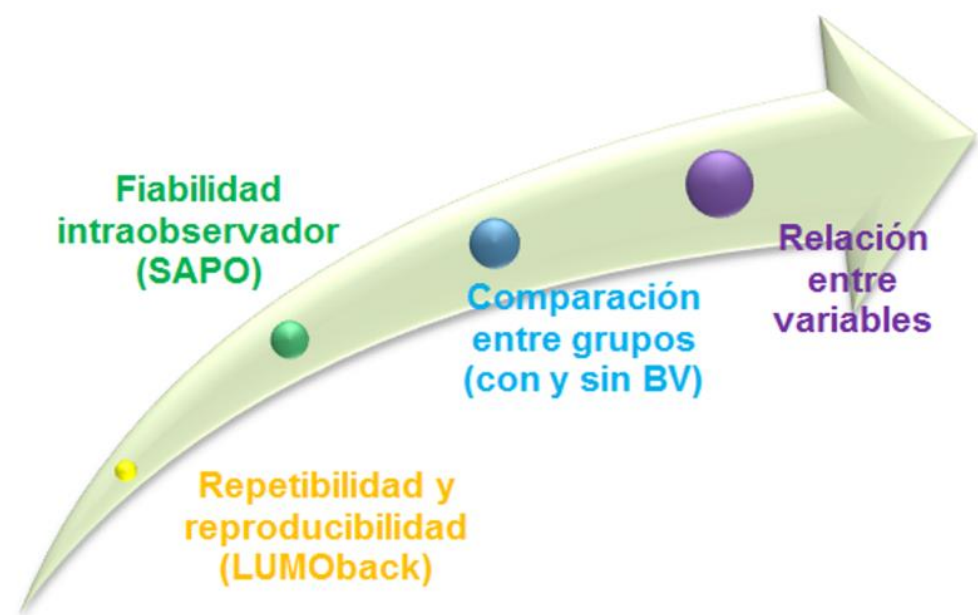

Figura 15: Fases del análisis de datos.

- Fase 1: Repetibilidad y reproducibilidad del LUMOback

La normalidad de las variables dependientes se analizó con la prueba de Shapiro Wilk. La fiabilidad intra e intersesión se analizó con el coeficiente de correlación intraclase $(\mathrm{CCl})$. La precisión del LUMOback se examinó siguiendo el procedimiento definido por Bland y Altman ${ }^{201}$. Para ello, se calcularon las BIAS (sesgo), su desviación típica (DT) y sus límites de confianza al 95\% (95\%LC). Por último, la asociación entre la diferencia y la magnitud de la medición (es decir, heterocedasticidad) se examinó mediante un análisis de regresión, introduciendo como variable dependiente 
la diferencia entre el ángulo lumbar medido por cada método y como variable independiente el valor promedio [(SAPO + LumoBack)/2]. La variabilidad intra e intersesión se calculó con el coeficiente de variación (CV; $\mathrm{CV}=\mathrm{DT}^{*} 100 /$ media). Las medias de las dos mediciones (día 1 y día 2, respectivamente) se compararon con una prueba de t pareada. Las medias de las tres mediciones del grupo de control obtenidas durante el día 1 se compararon con un análisis de varianza (ANOVA) para medidas repetidas. Con el fin de comprobar la validez concurrente, la relación entre los ángulos obtenidos por ambos métodos, el LUMOback y la fotogrametría con el SAPO, se analizó con el coeficiente de correlación de Pearson. Las correlaciones fueron interpretadas como alta $(>0.70)$, moderada $(0.50 \mathrm{a}$ $0.69)$, baja (0.26 a 0.49), y ausente $(0.00$ a 0.25$) 202$. Todos los valores se expresan como media (DT) y se estableció el nivel de significación $p<0.05$.

\section{- Fase 2: Fiabilidad intraobservador del SAPO}

Se evaluó el acuerdo entre días. Como primer paso se comprobó la hipótesis de que las diferencias entre mediciones proceden de una distribución normal, utilizando el contraste de normalidad de Shapiro-Wilk. Además, se evaluó el sesgo entre mediciones utilizando la prueba de contraste $t$ de Student para muestras pareadas y la relación lineal entre diferencia y media a partir del coeficiente de correlación de Pearson. El CCl fue utilizado para evaluar la proporción de la variabilidad de los sujetos y el porcentaje de variación respecto de la media fue evaluado a través del CV.

\section{- Fase 3: Comparación entre grupos (con y sin BV)}

Se comparó los dos grupos en cada una de las variables recogidas. Según el tipo de variable.

- Variables cuantitativas: se comprobó la hipótesis de normalidad utilizando el contraste de Shapiro-Wilk y la de homogeneidad de varianzas con el contraste de Brown-Forsythe. Posteriormente, se evaluó la hipótesis de igualdad de medias utilizando la prueba de contraste $t$ de Student para dos muestras independientes, en caso de cumplir los supuestos de normalidad y homogeneidad de varianza. En los casos en los que no erar posible asumir la normalidad de la variable dependiente, se utilizó el contraste $U$ de Mann-Whitney como alternativa no paramétrica. Se calcularon los estadísticos descriptivos, media, DT, el 95\% LC para la media, la mediana y los valores máximo (Max) y mínimo (Min).

- Variables cualitativas: se utilizó el contraste Chi-cuadrado para evaluar la hipótesis de independencia entre el grupo y la variable correspondiente, o el test exacto de Fisher cuando este contraste 
presentó problemas de convergencia. Los estadísticos descriptivos fueron los porcentajes de cada categoría junto con un límite de confianza del 95\%.

- Variables ordinales: se utilizó la misma metodología que para las variables cuantitativas en el caso de no-normalidad.

- Fase 4: Relación entre variables

La relación entre variables se dividió en dos partes: 1) la relación entre la VL y las variables: postura, equilibrio corporal, destreza manual, función visual y depresión; 2) la relación entra la depresión y las siguientes variables: postura, equilibrio corporal, destreza manual, miedo a caerse y dolor.

Para el análisis de la relación entre la VL y cada una de las variables se evaluó la relación de la variable dependiente VL con las variables independientes de interés en cada uno de los grupos por separado. Se hizo controlando los efectos de las variables SC, AV y tiempo de diagnóstico. Se distinguió el tipo de variable en:

- Variables independiente y dependiente cuantitativas: se evaluó el coeficiente de correlación de Spearman y un p-valor para contrastar la hipótesis de que la correlación era 0 , es decir que no había relación entre las dos variables.

- Variable independiente cualitativa y dependiente cuantitativa: se evaluó la igualdad de medias en la variable VL entre los grupos definidos por la variable correspondiente. En el caso de asumirse normalidad, se utilizó la prueba de contraste $t$ de Student para muestras independientes, y cuando no fue posible, se utilizó el contraste no paramétrico $U$ de Mann-Whitney.

Se realizaron el mismo tipo de análisis para evaluar la relación existente entre tener o no sintomatología depresiva según la escala GDS y cada una de las variables. Se consideró la variable GDS en dos grupos: no sintomatología depresiva y posible sintomatología depresiva.

Se utilizaron modelos de regresión lineal para evaluar si existía una relación, en cada uno de los grupos, entre la variable principal (VL) y cada una de las variables independientes de interés. 
5. RESULTADOS 



\subsection{Resultados Visuales}

\subsubsection{Agudeza Visual}

La AV en visión lejana se recogió en ambos ojos y se evaluó la AV media. La comparación entre grupos y comprobación de la hipótesis de normalidad y de homogeneidad de varianzas se describen en la Tabla 6.

Tabla 6: Datos descriptivos y contrastes de normalidad y homogeneidad de varianzas para la AV en ambos grupos.

\begin{tabular}{|c|c|c|c|c|c|c|c|c|}
\hline & \multirow[t]{2}{*}{$\mathbf{N}$} & \multirow[t]{2}{*}{ Media } & \multirow[t]{2}{*}{ DT } & \multirow[t]{2}{*}{ Mediana } & \multicolumn{2}{|c|}{$\begin{array}{c}\mathrm{H}_{0}: \\
\text { Normalidad }\end{array}$} & \multicolumn{2}{|c|}{$\begin{array}{c}\mathrm{H}_{0}: \\
\text { Homogeneidad } \\
\text { de varianzas (BF) }\end{array}$} \\
\hline & & & & & SW & p-valor & F valor & p-valor \\
\hline Caso & 31 & 1.04 & 0.46 & 1.03 & 0.945 & 0.1122 & & \\
\hline Control & 49 & 0.22 & 0.26 & 0.17 & 0.695 & $<0.0001$ & 19.78 & $<0.0001$ \\
\hline Total & 80 & 0.54 & 0.53 & 0.32 & 0.864 & $<0.0001$ & & \\
\hline
\end{tabular}

Al rechazar las hipótesis de normalidad y homogeneidad de varianzas, se utilizó el contraste $U$ de Mann-Whitney para la igualdad de medianas (Tabla 7).

Tabla 7: Contraste de igualdad de medianas para la AV en ambos grupos.

\begin{tabular}{|c|c|c|}
\hline \multirow{3}{*}{ AV $(\log M A R)$} & \multicolumn{2}{|c|}{$\begin{array}{c}\text { Ho: igualdad de medianas }_{\text {U de Mann-Whitney }}\end{array}$} \\
\hline & U & $\mathrm{p}$ - valor \\
\hline & 1469.5 & $<0.0001$ \\
\hline
\end{tabular}

Hubo diferencias estadísticamente significativas entre ambos grupos, siendo la $A V$ en logMAR significativamente mayor en el grupo caso $(p<0.0001)$.

\subsubsection{Sensibilidad al Contraste}

La SC fue recogida en tres variables: ojo derecho, izquierdo y binocular. Teniendo en cuenta que las tres variables estaban muy relacionadas entre sí (coeficiente de correlación de Spearman, $p<0.0001$ ), se eligió la variable binocular como representante. Se utilizó los contrastes de SW y BF para verificar la normalidad y la homogeneidad de varianzas de las muestras (Tabla 8). 
Tabla 8: Datos descriptivos y contrastes de normalidad y homogeneidad de varianzas para la SC en ambos grupos.

\begin{tabular}{|c|c|c|c|c|c|c|c|c|c|}
\hline & & \multirow[t]{2}{*}{$\mathbf{N}$} & \multirow[t]{2}{*}{ Media } & \multirow[t]{2}{*}{ DT } & \multirow[t]{2}{*}{ Mediana } & \multicolumn{2}{|c|}{$\begin{array}{c}\mathrm{H}_{0}: \\
\text { Normalidad }\end{array}$} & \multicolumn{2}{|c|}{$\begin{array}{c}\mathrm{H}_{0}: \\
\text { Homogeneidad } \\
\text { de varianzas } \\
\text { (BF) }\end{array}$} \\
\hline & & & & & & SW & $\mathrm{p}$-valor & $\mathrm{F}$ valor & $p$-valor \\
\hline \multirow{3}{*}{ SC } & Caso & 31 & 7.84 & 2.15 & 8 & 0.926 & 0.0346 & \multirow{3}{*}{1.46} & \multirow{3}{*}{0.231} \\
\hline & Control & 49 & 10.61 & 1.38 & 10 & 0.869 & 0.0001 & & \\
\hline & Total & 80 & 9.54 & 2.18 & 10 & 0.911 & $<0.0001$ & & \\
\hline
\end{tabular}

Al rechazar la hipótesis de normalidad en ambos grupos, la igualdad de medianas fue evaluada con el contraste $U$ de Mann-Whitney (Tabla 9).

Tabla 9: Contraste de igualdad de medianas para la SC en ambos grupos.

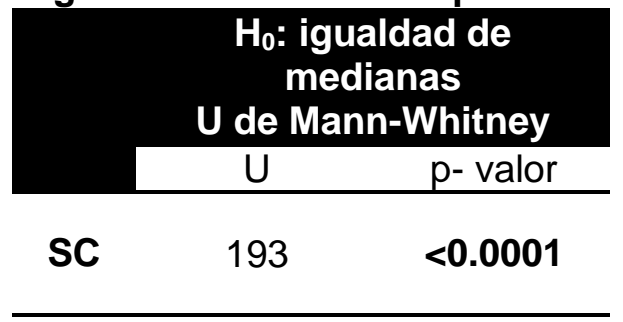

El valor de la SC fue significativamente menor en el grupo de los casos $(p<0.0001)$.

\subsubsection{Ayudas Ópticas y Patologías visuales}

La utilización de ayudas ópticas y las patologías visuales son variables encontradas solamente en el grupo de los casos. En lo que refiere a la utilización de ayudas ópticas para la lectura, de los 27 sujetos del grupo caso que participaron del IReST 21 hicieron uso de las mismas (Gráfico 1). Los datos referentes a las patologías visuales pueden ser observados en el Gráfico 2 . 
Gráfico 1: Distribución de la utilización de ayudas ópticas para lectura.

$\square$ Sí $\square$ No

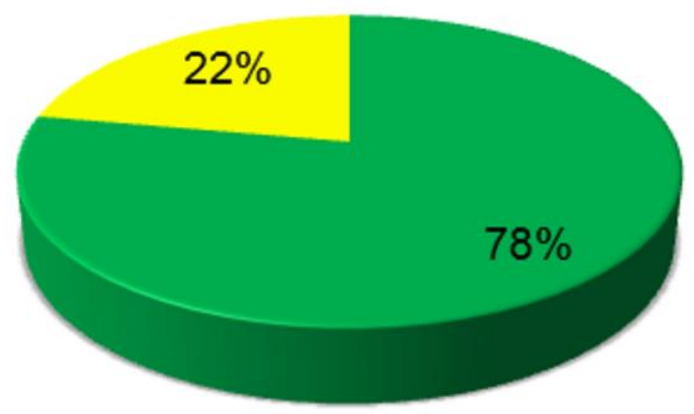

La mayoría de las personas con BV necesitaron utilizar alguna ayuda óptica para realizar la lectura (78\%).

Gráfico 2: Distribución por patología visual en el grupo caso (\%).

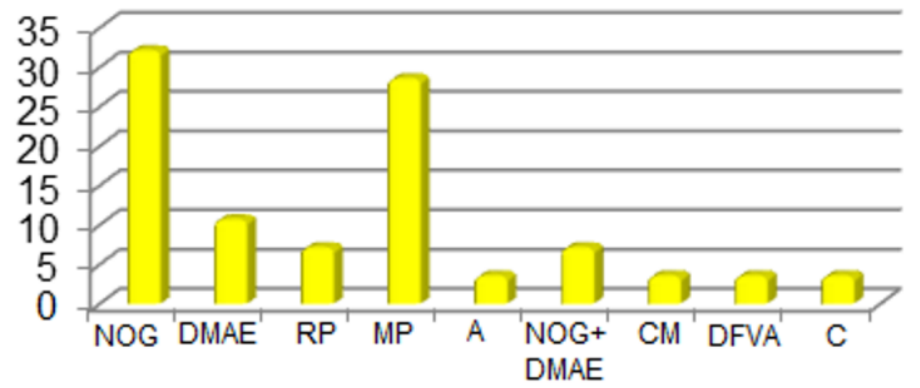

NOG: Neuropatía óptica glaucomatosa; DMAE: Degeneración macular asociada a la edad; RP: Retinosis pigmentaria; MP: Miopía patológica; A: Albinismo; CM: Coroidosis miópica; DFVA: Distrofia foveomacular viteliforme del adulto; C: Cataratas.

\subsection{Fiabilidad y validez del sensor postural LUMOback}

En lo que respecta a la fiabilidad intrasesión, la media del ángulo lumbar fue similar ( $p>0.05)$ entre las distintas mediciones realizadas el día 1 para cualquier grupo. Los sujetos del grupo control, así como los casos, mostraron una excelente repetibilidad intrasesión y una baja variabilidad (Tabla 10). La fiabilidad intersesión también fue excelente mostrando una variabilidad reducida (Tabla 10). No hubo diferencia estadísticamente significativa en los valores del ángulo lumbar entre las medidas obtenidas el día 1 y 2. 
Tabla 10: Resultados de la repetibilidad intrasesión.

\begin{tabular}{|c|c|c|c|c|c|c|c|}
\hline \multicolumn{2}{|c|}{ Grupo } & Media & $\mathbf{D T}^{\mathrm{a}}$ & \multicolumn{2}{|c|}{$\mathrm{CCl}^{\mathrm{b}}$} & \multicolumn{2}{|c|}{$\mathbf{C V}^{\mathrm{c}}$} \\
\hline & & & & Intrasesión & Intersesión & Intrasesión & Intersesión \\
\hline \multirow{2}{*}{ Control } & $\begin{array}{c}\text { Día } \\
1\end{array}$ & 98.6 & 8.6 & $0.84^{*}$ & \multirow{2}{*}{$0.89^{*}$} & $2.7 \%$ & \multirow{2}{*}{$5.72 \%$} \\
\hline & $\begin{array}{c}\text { Día } \\
2\end{array}$ & 99.0 & 10.7 & $0.85^{*}$ & & $1.9 \%$ & \\
\hline Caso & & 98.3 & 10.3 & $0.94^{*}$ & - & $1.9 \%$ & - \\
\hline
\end{tabular}

El gráfico de Bland y Altman para el grupo control indicó que el sesgo fue de $0.04^{\circ}$ (3.07) con LA de $95 \%$ entre $-5.98^{\circ}$ y $6.07^{\circ}$. Por otro lado, para el grupo caso el sesgo fue de $0.75^{\circ}$ (1.78) con LC entre $-2.73^{\circ}$ y $4.24^{\circ}$. El análisis heterocedasticidad mostró una asociación no significativa estadísticamente para los sujetos del grupo control $\left(R^{2}=0.040, p=0.221\right)$ y para los sujetos del grupo caso $\left(R^{2}=0.092, p=0.149\right)$. El sesgo para todos los sujetos fue $0.31^{\circ}$ (2.66) con LC de $-4.90^{\circ}$ y $5.53^{\circ}$ (Gráfico 3). El análisis de heterocedasticidad mostró una asociación no significativa estadísticamente $\left(R^{2}=0.048, p=0.084\right)$ entre la diferencia absoluta y el promedio del ángulo lumbar medido con ambos sistemas para todos los sujetos.

En cuanto a la validez concurrente, la correlación con el SAPO fue fuerte para los controles $(r=0.934, p<0.001)$ y para los casos $(r=0.985, p<0.001)$ (Gráfico 4). La comparación de los valores obtenidos con ambos sistemas no mostró diferencias estadísticamente significativas $(p=0.349)$.

Gráfico 3: Acuerdo de medición entre los ángulos lumbares registrados con el SAPO y el LUMOback.

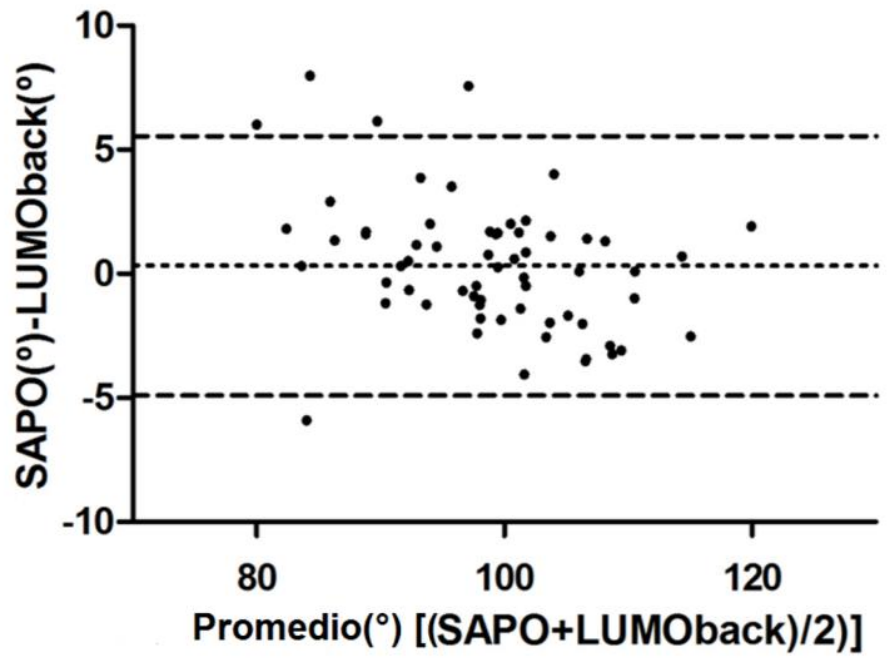


Gráfico 4: Correlación entre los valores de los ángulos lumbares medidos por el LUMOback y el SAPO en ambos grupos.

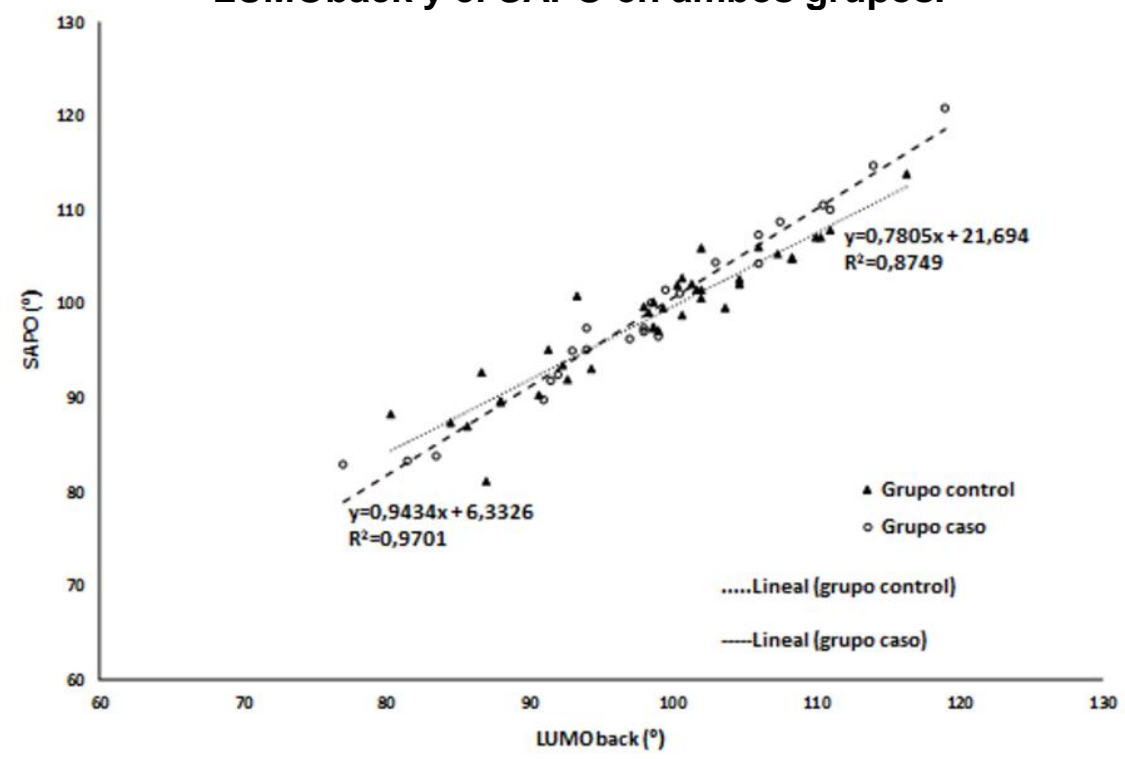

\subsection{Fiabilidad intraobservador de las medidas posturales utilizando el protocolo SAPO}

Teniendo en cuenta que la hipótesis de normalidad fue evaluada anteriormente y que se encontró una distribución normal, se evaluó la igualdad de medias entre días con el t-Student. En la Tabla 11 están representados los datos de las medias, DT, CCI, CV y el t-Student de los ocho ángulos medidos.

Tabla 11: Datos estadísticos del acuerdo entre días de las medidas posturales.

\begin{tabular}{|c|c|c|c|c|c|c|}
\hline & Medida & Media & DT & CCI & CV & $\begin{array}{c}\text { T-Student } \\
\text { p-valor }\end{array}$ \\
\hline \multirow{2}{*}{${ }^{a} \mathbf{F C}$} & 1 & 70.40 & 7.94 & \multirow{2}{*}{1.00} & \multirow{2}{*}{0.14} & \multirow{2}{*}{0.8669} \\
\hline & 2 & 70.39 & 7.97 & & & \\
\hline \multirow{2}{*}{${ }^{\mathrm{b}} \mathrm{Fcu}$} & 1 & 44.07 & 8.66 & \multirow{2}{*}{0.99} & \multirow{2}{*}{0.42} & \multirow{2}{*}{0.0825} \\
\hline & 2 & 43.97 & 8.62 & & & \\
\hline \multirow{2}{*}{${ }^{\circ} A C C$} & 1 & 152.22 & 12.13 & \multirow{2}{*}{1.00} & \multirow{2}{*}{0.06} & \multirow{2}{*}{0.4409} \\
\hline & 2 & 152.23 & 12.17 & & & \\
\hline \multirow{2}{*}{${ }^{\mathrm{d}} \mathbf{A C T}$} & 1 & 145.93 & 8.65 & \multirow{2}{*}{1.00} & \multirow{2}{*}{0.07} & \multirow{2}{*}{0.2959} \\
\hline & 2 & 145.97 & 8.62 & & & \\
\hline \multirow{2}{*}{ eFT } & 1 & 9.93 & 4.55 & \multirow{2}{*}{0.98} & \multirow{2}{*}{6.57} & \multirow{2}{*}{0.7550} \\
\hline & 2 & 9.84 & 4.60 & & & \\
\hline \multirow{2}{*}{${ }^{f} \mathbf{A} T$} & 1 & 219.90 & 7.42 & \multirow{2}{*}{1.00} & \multirow{2}{*}{0.05} & \multirow{2}{*}{0.2499} \\
\hline & 2 & 219.94 & 7.41 & & & \\
\hline \multirow{2}{*}{${ }^{9} \mathbf{A L}$} & 1 & 88.70 & 14.22 & \multirow{2}{*}{0.99} & \multirow{2}{*}{0.43} & \multirow{2}{*}{0.4072} \\
\hline & 2 & 88.76 & 14.46 & & & \\
\hline \multirow{2}{*}{ hIP } & 1 & 33.66 & 13.41 & \multirow{2}{*}{1.00} & \multirow{2}{*}{0.64} & \multirow{2}{*}{0.7699} \\
\hline & 2 & 33.63 & 13.46 & & & \\
\hline
\end{tabular}

aFlexión de la cabeza; 'bFlexión del cuello; 'Ángulo cráneo-cervical; dÁngulo cérvico-torácico;

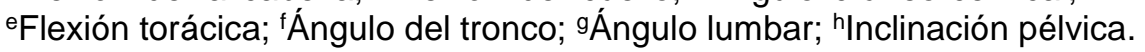


No hubo diferencias estadísticamente significativas entre las dos mediciones en ninguno de los ángulos medidos $(p>0.05)$, con una excelente repetibilidad intersesión ( $\mathrm{CCl}>0.95)$.

\subsection{Resultados Posturales}

\subsubsection{Evaluación postural en sedestación}

Para comprobar la normalidad y homogeneidad de varianza entre las poblaciones estudiadas, se utilizó el contraste de SW y el de Brown-Forsythe (BF), respectivamente (Tabla 12).

Tabla 12: Datos descriptivos y contrastes de normalidad y homogeneidad de varianzas para los ángulos posturales en sedestación en ambos grupos.

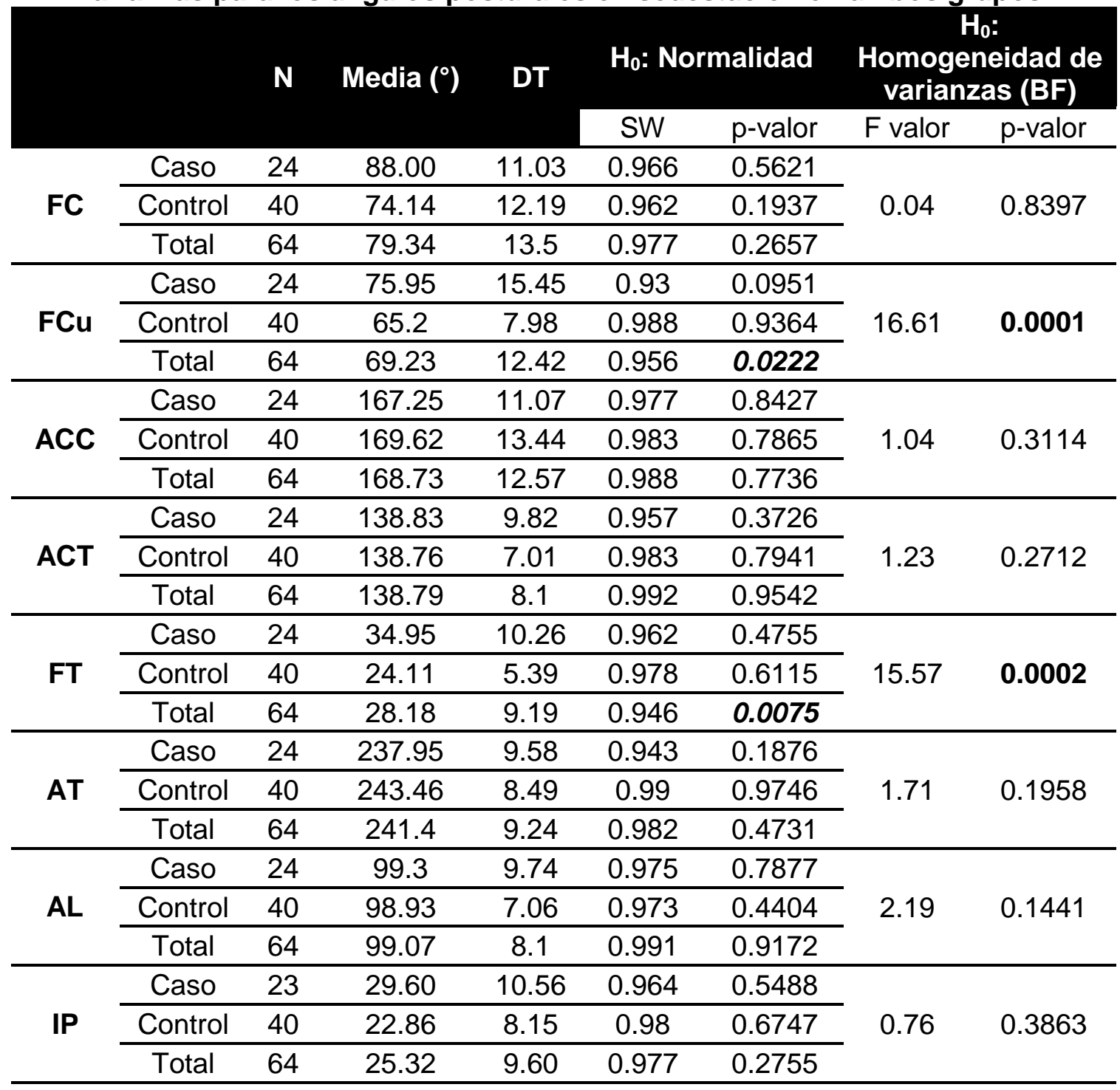


No se rechazó la hipótesis de normalidad en ninguno de los grupos y se rechazó la hipótesis de homogeneidad de varianzas en la FCu y en la FT, con variabilidad mayor en el grupo caso. La igualdad de medias fue evaluada utilizando el contraste t-Student (Tabla 13).

Tabla 13: Contraste de igualdad de medias para los ángulos posturales en sedestación.

\begin{tabular}{ccc} 
& \multicolumn{2}{c}{$\begin{array}{c}\text { Ho: igualdad de } \\
\text { medias } \\
\text { t-Student }\end{array}$} \\
& t-test & $\mathrm{p}$-valor \\
\hline FC & 4.558 & $<\mathbf{0 . 0 0 0 1}$ \\
\hline FCu & 3.166 & $\mathbf{0 . 0 0 3 5}$ \\
\hline ACC & -0.727 & 0.47 \\
\hline ACT & 0.034 & 0.9733 \\
\hline FT & 4.794 & $<\mathbf{0 . 0 0 0 1}$ \\
\hline AT & -2.393 & $\mathbf{0 . 0 1 9 8}$ \\
\hline AL & 0.176 & 0.8612 \\
\hline IP & 2.834 & $\mathbf{0 . 0 0 6 2}$ \\
\hline
\end{tabular}

Al comparar las medias para cada ángulo postural medido entre los dos grupos se encontraron diferencias estadísticamente significativas, siendo los valores significativamente mayores en el grupo caso para la flexión de cabeza, de cuello, de tórax y la inclinación pélvica, en contrapartida el ángulo del tronco fue significativamente menor en el grupo caso. Dichos datos están representados en las Figuras 16, 17, 18, 19 y 20, respectivamente.

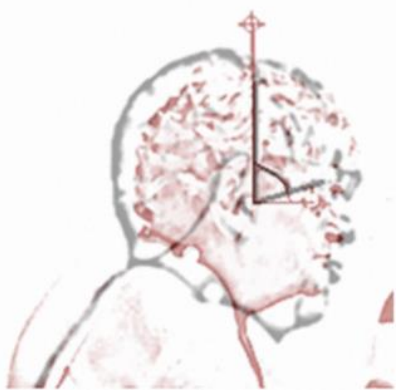

Figura 16: FC de los grupos caso (en rojo) y control (en negro). 


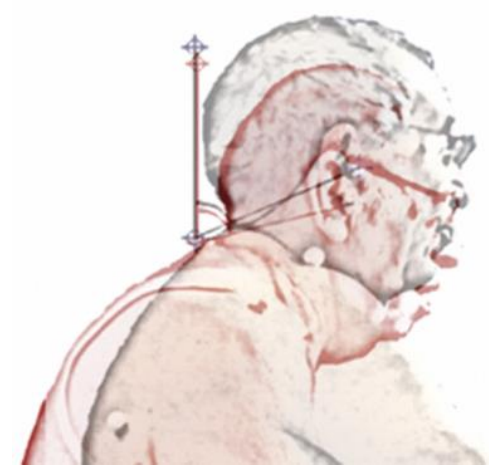

Figura 17: FCu de los grupos caso (en rojo) y control (en negro).

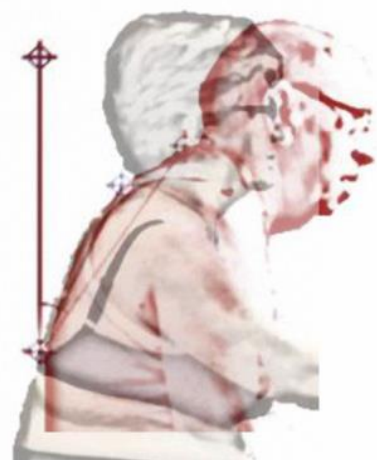

Figura 18: FT de los grupos caso (en rojo) y control (en negro).

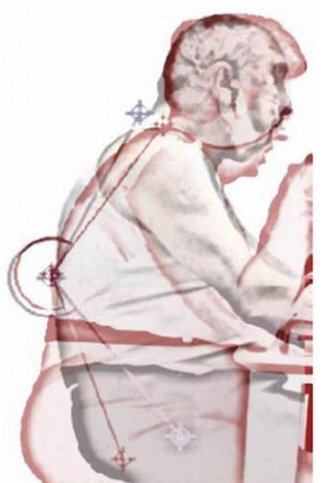

Figura 19: AT de los grupos caso (en rojo) y control (en negro).

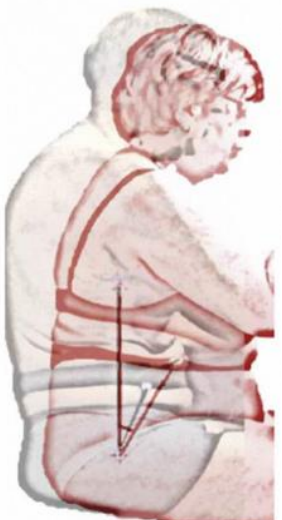

Figura 20: IP de los grupos caso (en rojo) y control (en negro). 


\subsubsection{Evaluación postural en bipedestación}

Para comprobar la normalidad y homogeneidad de varianza entre las poblaciones estudiadas, se utilizó el contraste de SW y el de BF, respectivamente (Tabla 14).

Tabla 14: Datos descriptivos y contrastes de normalidad y homogeneidad de varianzas para los ángulos posturales en bipedestación en ambos grupos.

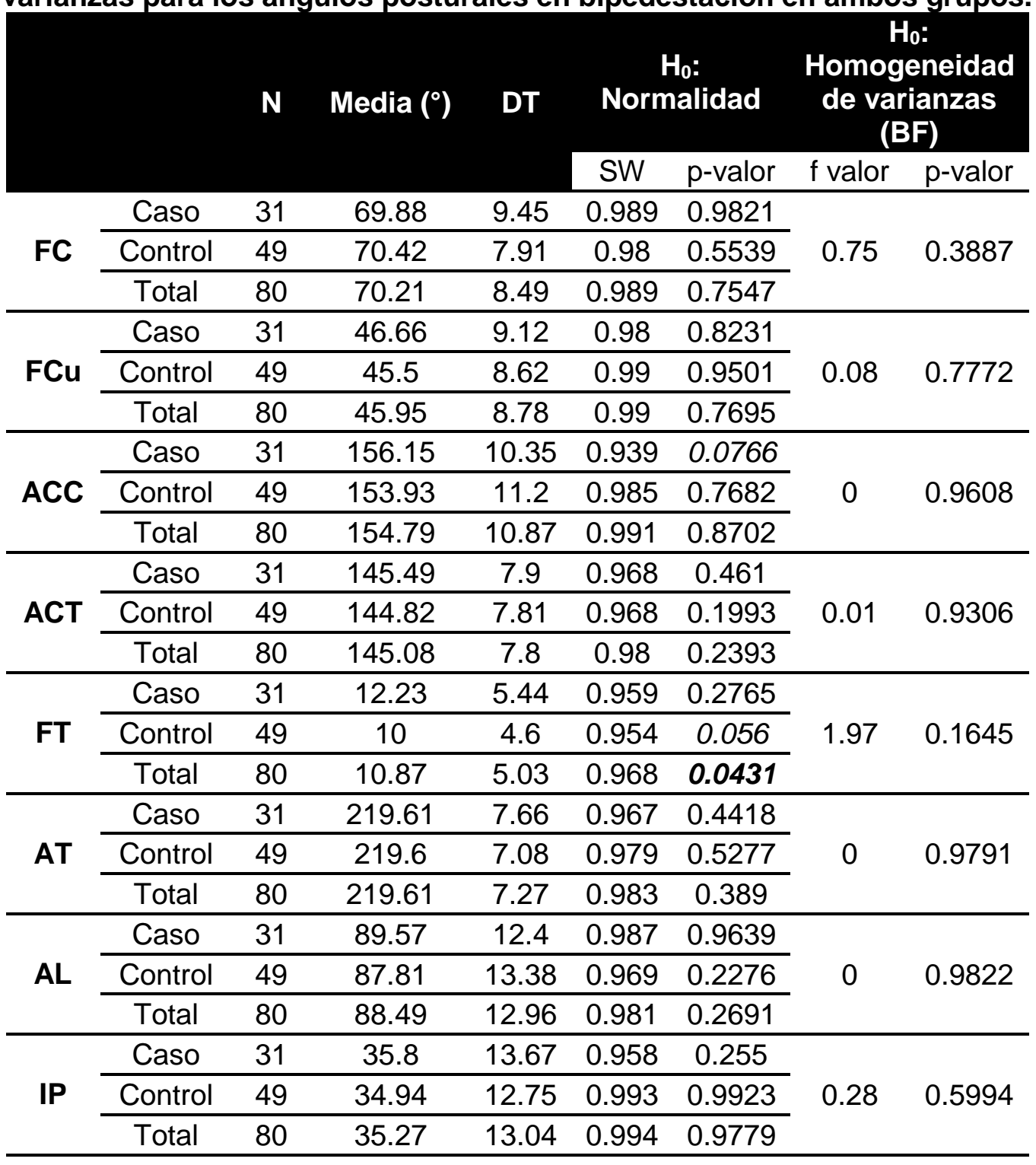


No se rechazó la hipótesis de normalidad ni la de homogeneidad de varianzas, con lo cual se utilizó el contraste t-Student para verificar la igualdad de medias entre los dos grupos para la variable postura en bipedestación (Tabla 15).

Tabla 15: Contraste de igualdad de medias para los ángulos posturales en bipedestación.

\begin{tabular}{ccc} 
& \multicolumn{2}{c}{$\begin{array}{c}\text { Ho: igualdad de } \\
\text { medias } \\
\text { t-Student }\end{array}$} \\
& t-test & $\mathrm{p}$-valor \\
\hline FC & -0.277 & 0.7828 \\
\hline FCu & 0.573 & 0.5686 \\
\hline ACC & 0.888 & 0.3774 \\
\hline ACT & 0.372 & 0.7109 \\
\hline FT & 1.969 & 0.0525 \\
\hline AT & 0.003 & 0.9973 \\
\hline AL & 0.59 & 0.5567 \\
\hline IP & 0.287 & 0.775 \\
\hline
\end{tabular}

No hubo diferencias estadísticamente significativas entre los dos grupos ( $p>0.05$ ) en ninguno de los ángulos medidos, a pesar de que para la FT el $p$ valor se quedó al borde de la significación, siendo mayor en el grupo caso $(p=0.0525)$.

\subsection{Resultados de las demás variables físicas}

\subsubsection{Equilibrio}

La hipótesis de normalidad y homogeneidad de varianzas fue analizada con los contrastes de SW y BF, respectivamente (Tabla 16).

Tabla 16: Datos descriptivos y contrastes de normalidad y homogeneidad de varianzas para el equilibrio en ambos grupos.

\begin{tabular}{|c|c|c|c|c|c|c|c|c|}
\hline & \multirow[t]{2}{*}{$\mathbf{N}$} & \multirow[t]{2}{*}{$\begin{array}{c}\text { Media } \\
\left(^{*}\right)\end{array}$} & \multirow[t]{2}{*}{ DT } & \multirow[t]{2}{*}{ Mediana } & \multicolumn{2}{|c|}{$\begin{array}{c}\mathrm{H}_{0}: \\
\text { Normalidad }\end{array}$} & \multicolumn{2}{|c|}{$\begin{array}{c}\mathrm{H}_{0}: \\
\text { Homogeneidad } \\
\text { de varianzas } \\
\text { (BF) }\end{array}$} \\
\hline & & & & & SW & $\mathrm{p}$-valor & $\mathrm{F}$ valor & p-valor \\
\hline Caso & 31 & 11.87 & 3.62 & 11 & 0.921 & 0.0252 & & \\
\hline Control & 49 & 10.78 & 2.89 & 10 & 0.914 & 0.0016 & 1.86 & 0.1761 \\
\hline Total & 80 & 11.2 & 3.22 & 10 & 0.918 & 0.0001 & & \\
\hline
\end{tabular}

*Valores en segundos (s). 
Debido a la distribución no-normal de la variable evaluada, se utilizó el contraste U de Mann-Whitney para la igualdad de medianas (Tabla 17).

Tabla 17: Contraste de igualdad de medianas para el equilibrio.

\begin{tabular}{|c|c|c|}
\hline \multirow[t]{2}{*}{ Grupos } & \multicolumn{2}{|c|}{$\begin{array}{c}\mathrm{H}_{0} \text { : igualdad de } \\
\text { medianas } \\
\text { U de Mann-Whitney }\end{array}$} \\
\hline & $U$ & $p$ - valor \\
\hline $\begin{array}{c}\text { Caso } \\
\text { Control }\end{array}$ & 879.5 & 0.2348 \\
\hline
\end{tabular}

De acuerdo con tales resultados, no hubo diferencias estadísticamente significativas en el equilibrio entre los dos grupos.

\subsubsection{Destreza manual}

Para los datos de la destreza manual, elegimos el mejor tiempo (en segundos) de los tres intentos en cada mano con los ojos abiertos y cerrados. Para comprobar la hipótesis de normalidad y homogeneidad de varianzas fueron utilizados los contrastes de SW y BF, respectivamente (Tabla 18).

Tabla 18: Datos descriptivos y contrastes de normalidad y homogeneidad de varianzas para la destreza manual en ambos grupos.

\begin{tabular}{|c|c|c|c|c|c|c|c|c|c|}
\hline & & \multirow[t]{2}{*}{$\mathbf{N}$} & \multirow[t]{2}{*}{$\begin{array}{c}\text { Media } \\
\left(^{\circ}\right)\end{array}$} & \multirow[t]{2}{*}{ DT } & \multirow[t]{2}{*}{ Mediana } & \multicolumn{2}{|c|}{$\mathrm{H}_{0}$ : Normalidad } & \multicolumn{2}{|c|}{$\begin{array}{c}\mathrm{H}_{0}: \\
\text { Homogeneidad } \\
\text { de varianzas } \\
\text { (BF) }\end{array}$} \\
\hline & & & & & & SW & $\mathrm{p}$-valor & $\begin{array}{c}\mathrm{F} \\
\text { valor }\end{array}$ & $p$-valor \\
\hline \multirow{3}{*}{${ }^{\mathrm{a} M D / O A}$} & Caso & 31 & 14.06 & 2.93 & 14 & 0.943 & 0.1014 & \multirow{3}{*}{4.42} & \multirow{3}{*}{0.0386} \\
\hline & Control & 49 & 11.67 & 2.13 & 11 & 0.833 & $<0.0001$ & & \\
\hline & Total & 80 & 12.6 & 2.72 & 12 & 0.886 & $<0.0001$ & & \\
\hline \multirow{3}{*}{${ }^{b} \mathrm{MI} / \mathrm{OA}^{\mathrm{c}}$} & Caso & 31 & 15.23 & 4.56 & 14 & 0.854 & 0.0006 & \multirow{3}{*}{13.32} & \multirow{3}{*}{0.0005} \\
\hline & Control & 49 & 12.12 & 1.67 & 12 & 0.944 & 0.0203 & & \\
\hline & Total & 80 & 13.32 & 3.45 & 13 & 0.786 & $<0.0001$ & & \\
\hline \multirow{3}{*}{$M D / O C^{d}$} & Caso & 31 & 25.48 & 8.86 & 24 & 0.863 & 0.001 & \multirow{3}{*}{1.41} & \multirow{3}{*}{0.2391} \\
\hline & Control & 49 & 26.37 & 7.15 & 26 & 0.847 & $<0.0001$ & & \\
\hline & Total & 80 & 26.02 & 7.81 & 26 & 0.864 & $<0.0001$ & & \\
\hline \multirow{3}{*}{$\mathrm{MI} / \mathrm{OC}$} & Caso & 31 & 27.48 & 8.57 & 25 & 0.926 & 0.0347 & \multirow{3}{*}{3.77} & \multirow{3}{*}{0.0558} \\
\hline & Control & 49 & 28.51 & 5.75 & 28 & 0.968 & 0.2027 & & \\
\hline & Total & 80 & 28.11 & 6.94 & 27 & 0.96 & 0.0132 & & \\
\hline
\end{tabular}

aMano derecha; ${ }^{\mathrm{b} M a n o}$ izquierda; ' $\mathrm{O}$ jos abiertos; ' $\mathrm{O}$ jos cerrados. 
Se rechazó la hipótesis de normalidad y la de homogeneidad de varianzas, con lo cual se utilizó el contraste $U$ de Mann-Whitney para evaluar la igualdad de medianas (Tabla 19).

Tabla 19: Contraste de igualdad de medianas en la evaluación de la destreza manual.

\begin{tabular}{ccc} 
& \multicolumn{2}{c}{ Ho: igualdad de medianas U de Mann-Whitney } \\
& $\mathrm{U}$ & $\mathrm{p}$ - valor \\
\hline MD/OA & 1169 & $\mathbf{< 0 . 0 0 0 1}$ \\
\hline MI/OA & 1120 & $\mathbf{0 . 0 0 0 3}$ \\
\hline MD/OC & 655 & 0.3035 \\
\hline MI/OC & 634.5 & 0.2183 \\
\hline
\end{tabular}

La diferencia entre los grupos en el valor del Pick Up test en ambas manos con los ojos abiertos fue estadísticamente significativa, siendo significativamente mayor en el grupo de los casos, lo que significa que las personas con BV tardaron más tiempo para realizar dicha prueba. Sin embargo, no hubo diferencias significativas estadísticamente entre los dos grupos al realizar la misma prueba con los ojos cerrados.

\subsubsection{Velocidad lectora}

En lo que se refiere a la VL, los resultados del IReST fueron expresados en ppm. Del total de participantes, 5 (4 del grupo caso y 1 del grupo control) no sabían leer, razón por la cual no participaron de dicho test. Así que, la muestra para esta evaluación fue compuesta de 27 sujetos en el grupo caso y 48 en el grupo control.

La hipótesis de normalidad y homogeneidad de varianzas fueron comprobadas con los contrastes de SW y BF, respectivamente y sus resultados juntamente con los datos descriptivos de la VL en ambos grupos están expresados en la Tabla 20. 
Tabla 20: Datos descriptivos y contrastes de normalidad y homogeneidad de varianzas para la VL en ambos grupos.

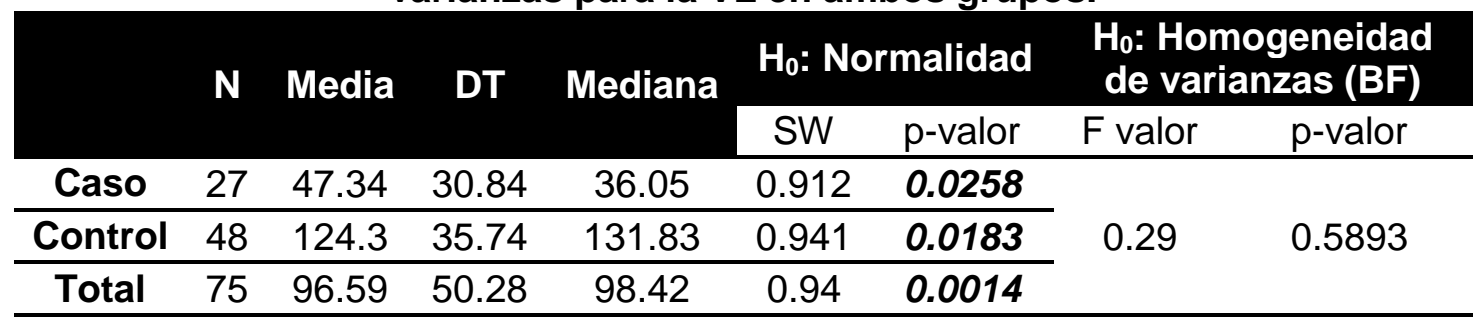

Teniendo en cuenta que la hipótesis de normalidad fue rechazada, para evaluar la igualdad de medianas se utilizó el contraste U de Mann-Whitney (Tabla 21).

Tabla 21: Contraste de igualdad de medianas en la evaluación de la VL.

\begin{tabular}{ccc} 
& \multicolumn{2}{c}{$\begin{array}{c}\text { Ho: igualdad de } \\
\text { medianas }\end{array}$} \\
& U de Mann-Whitney \\
& $U$ & p-valor \\
\hline VL & 85.5 & $<\mathbf{0 . 0 0 0 1}$ \\
\hline
\end{tabular}

Hubo una diferencia estadísticamente significativa en la VL entre los dos grupos, siendo el valor de la variable IReST significativamente menor en el grupo caso, es decir las personas del grupo caso leyeron menos palabras por minuto que las del grupo control (Gráfico 5).

Gráfico 5: Distribución de la VL en cada grupo.

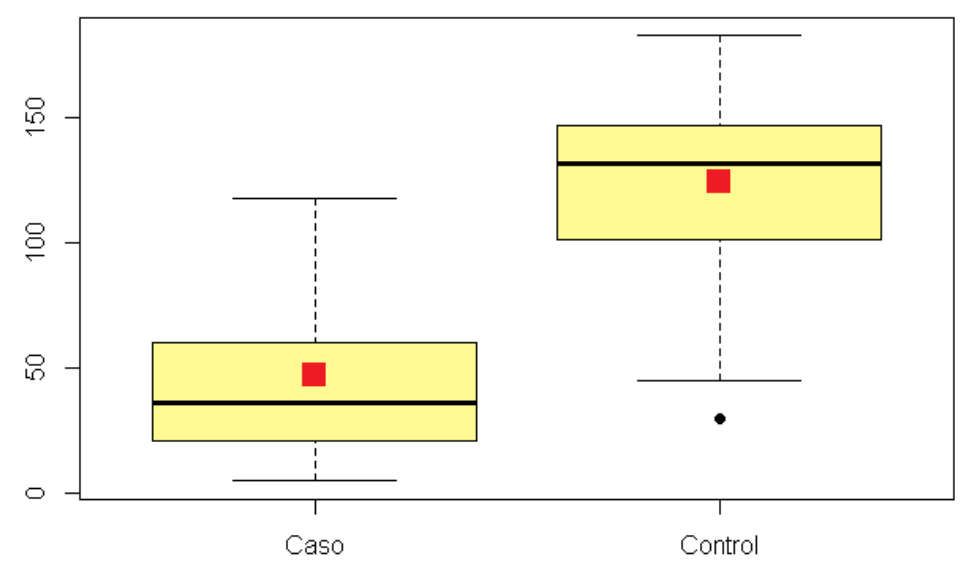

\subsection{Resultados Psicológicos}

\subsubsection{Depresión}

Se analizó la hipótesis de normalidad y la de homogeneidad de varianzas utilizando los contrastes SW y BF, respectivamente (Tabla 22). 
Tabla 22: Datos descriptivos y contrastes de normalidad y homogeneidad de varianzas para la sintomatología depresiva en ambos grupos.

\begin{tabular}{|c|c|c|c|c|c|c|c|c|}
\hline & \multirow[t]{2}{*}{$\mathbf{N}$} & \multirow[t]{2}{*}{ Media } & \multirow[t]{2}{*}{ DT } & \multirow[t]{2}{*}{ Mediana } & \multicolumn{2}{|c|}{$\begin{array}{c}\mathrm{H}_{0}: \\
\text { Normalidad }\end{array}$} & \multicolumn{2}{|c|}{$\begin{array}{c}\mathrm{H}_{0}: \\
\text { Homogeneidad } \\
\text { de varianzas } \\
\text { (BF) }\end{array}$} \\
\hline & & & & & SW & p-valor & F valor & $p$-valor \\
\hline Caso & 31 & 3.45 & 3.09 & 2 & 0.877 & 0.0021 & & \\
\hline Control & 49 & 2.49 & 2.94 & 1 & 0.799 & $<0.0001$ & 0.56 & 0.4575 \\
\hline Total & 80 & 2.86 & 3.01 & 2 & 0.845 & $<0.0001$ & & \\
\hline
\end{tabular}

Debido a la no-normalidad de la muestra, se utilizó el contraste $U$ de MannWhitney para contrastar la igualdad de medianas entre los dos grupos (Tabla 23).

Tabla 23: Contraste de igualdad de medianas para la sintomatología depresiva en ambos grupos.

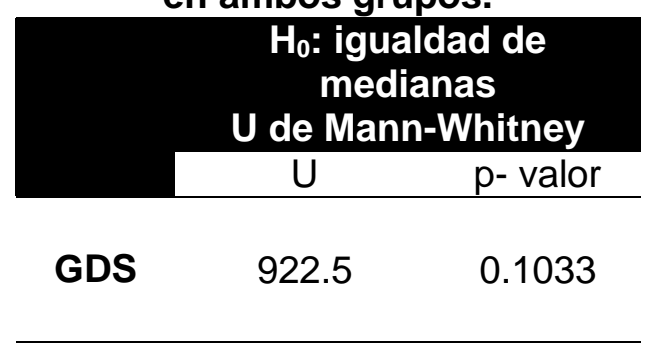

No se encontró diferencias estadísticamente significativas en la sintomatología depresiva entre ambos grupos.

\subsubsection{Miedo a caerse}

La normalidad y la homogeneidad de varianzas de la muestra fueron verificadas con los contrastes de SW y BF, respectivamente (Tabla 24).

Tabla 24: Datos descriptivos y contrastes de normalidad y homogeneidad de varianzas para el miedo a caerse en ambos grupos.

\begin{tabular}{|c|c|c|c|c|c|c|c|c|}
\hline & \multirow[t]{2}{*}{$\mathbf{N}$} & \multirow[t]{2}{*}{ Media } & \multirow[t]{2}{*}{ DT } & \multirow[t]{2}{*}{ Mediana } & \multicolumn{2}{|c|}{$\begin{array}{c}\mathrm{H}_{0}: \\
\text { Normalidad }\end{array}$} & \multicolumn{2}{|c|}{$\begin{array}{c}\mathrm{H}_{0}: \\
\text { Homogeneidad } \\
\text { de varianzas } \\
\text { (BF) }\end{array}$} \\
\hline & & & & & SW & p-valor & F valor & $p$-valor \\
\hline Caso & 31 & 22.58 & 5.99 & 21 & 0.694 & $<0.0001$ & \multirow{3}{*}{0.59} & \multirow{3}{*}{0.4436} \\
\hline Control & 49 & 19.76 & 4.09 & 18 & 0.822 & $<0.0001$ & & \\
\hline Total & 80 & 20.85 & 5.07 & 20 & 0.772 & $<0.0001$ & & \\
\hline
\end{tabular}


Se rechazó la hipótesis de normalidad de la muestra, por tanto, se utilizó el contraste $U$ de Mann-Whitney para evaluar la igualdad de medianas (Tabla 25).

Tabla 25: Contraste de igualdad de medianas para el miedo a caerse en ambos grupos.

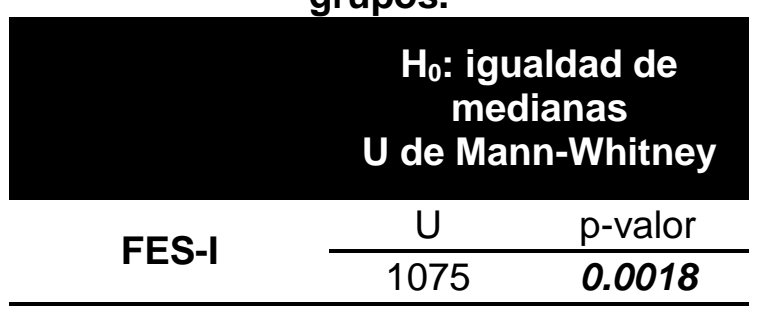

El miedo a caerse fue significativamente mayor en el grupo caso, siendo tal diferencia estadísticamente significativa.

\subsubsection{Dolor}

El dolor percibido en ambos grupos fue evaluado a través de la escala EVA para diferentes partes del cuerpo. Para verificar la hipótesis de normalidad y de homogeneidad de varianzas se utilizó los contrastes de SW y BF, respectivamente (Tabla 26 ). 
Tabla 26: Datos descriptivos y contrastes de normalidad y homogeneidad de varianzas para el dolor en ambos grupos.

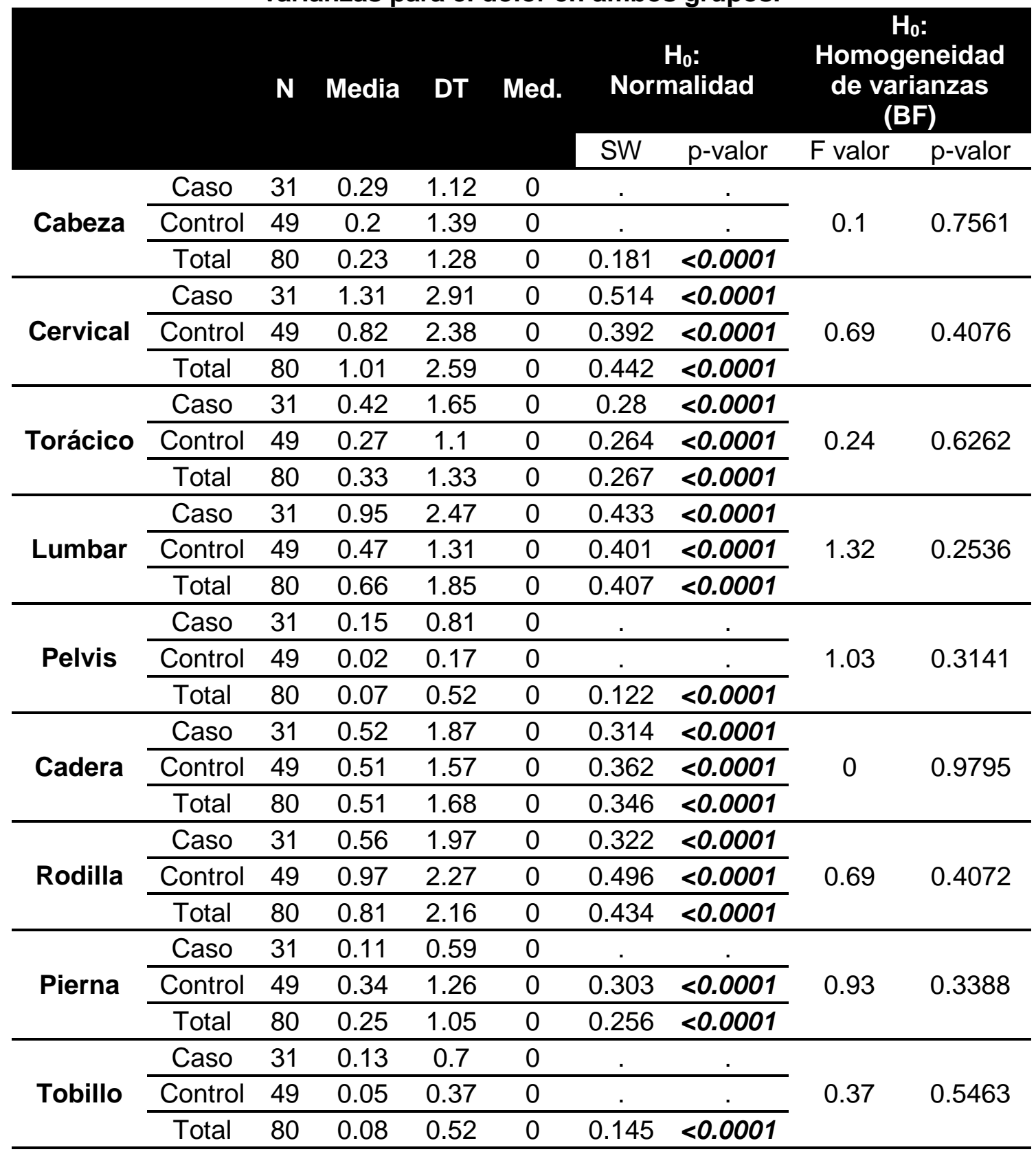

Se rechazó las hipótesis de normalidad en todas las partes del cuerpo evaluadas, así que se evaluó la igualdad de medianas con el contraste $\mathrm{U}$ de Mann-Whitney (Tabla 27). 
Tabla 27: Contraste de igualdad de medianas para el dolor en ambos grupos.

\begin{tabular}{lcc} 
& \multicolumn{2}{c}{$\begin{array}{c}\text { Ho: } \text { medianas } \\
\text { median }\end{array}$} \\
& U de Mann-Whitney \\
\hline Cabeza & 792 & 0.337 \\
\hline Cervical & 802 & 0.5185 \\
\hline Tórax & 787 & 0.5593 \\
\hline Lumbar & 797 & 0.5416 \\
\hline Pelvis & 769 & 0.7424 \\
\hline Cadera & 754 & 0.9244 \\
\hline Rodilla & 679.5 & 0.2216 \\
\hline Pierna & 721 & 0.3711 \\
\hline Tobillo & 769 & 0.7424 \\
\hline
\end{tabular}

No se encontraron diferencias estadísticamente significativas entre los dos grupos en ninguna de las regiones del cuerpo evaluadas.

\subsection{Resultados de la correlación entre las variables}

Teniendo en cuenta que la variable VL no cumple el supuesto de normalidad (Tabla 20), se utilizó el coeficiente no paramétrico de correlación de Spearman para evaluar la relación entre la VL y todas las variables de interés.

5.7.1. Relación entre la VL y la Postura Corporal

5.7.1.1. VL y FC

La relación entre la VL y la FC puede verse en la Tabla 28.

Tabla 28: Correlación entre la VL y la FC.

\begin{tabular}{cccc} 
& & Rho de Spearman & p-valor rho=0 \\
\multirow{3}{*}{ FC } & Control & 0.231 & 0.1515 \\
\cline { 2 - 4 } & Caso & -0.2431 & 0.2636 \\
\cline { 2 - 4 } & Total & -0.3165 & $\mathbf{0 . 0 1 1 5}$ \\
\hline
\end{tabular}


En el grupo control la correlación fue positiva, es decir que mayor ángulo de FC se asoció con menor VL y en el grupo caso la correlación fue negativa. En ninguno de los dos casos la relación fue estadísticamente significativa.

La influencia del ángulo postural FC sobre la VL controlando las variables de confusión SC, AV y tiempo de diagnóstico (la última variable sólo está disponible para el grupo caso) fue evaluada con el modelo de regresión lineal (Tabla 29).

Tabla 29: Modelo de regresión lineal - VL y FC.

\begin{tabular}{|c|c|c|c|c|c|c|c|c|}
\hline & \multirow{2}{*}{$\widehat{b}_{i}$} & \multirow{2}{*}{ EE } & \multicolumn{2}{|c|}{ IC $95 \%$ para $\widehat{b}_{i}$} & \multicolumn{2}{|c|}{$\mathrm{H}_{0}: \widehat{\boldsymbol{b}}_{\boldsymbol{i}}=\mathbf{0}$} & \multirow{2}{*}{ VIF } \\
\hline & & & & Inf. & Sup. & t-valor & $\mathrm{p}$-valor & \\
\hline \multirow{4}{*}{ Control } & (Intercept) & 63.733 & 48.295 & -34.2138 & 161.6796 & 1.32 & 0.1953 & \\
\hline & $\mathrm{SC}$ & 3.164 & 4.54 & $\begin{array}{l}-6.0426 \\
\end{array}$ & 12.371 & 0.697 & 0.4903 & 1.4036 \\
\hline & AV & 8.339 & 23.942 & -40.2177 & 56.8951 & 0.348 & 0.7297 & 1.4375 \\
\hline & $\mathrm{X}: \mathrm{FC}$ & 0.353 & 0.54 & -0.7417 & 1.4486 & 0.655 & 0.5169 & 1.3747 \\
\hline \multirow{5}{*}{ Caso } & (Intercept) & 84.426 & 68.028 & -58.4949 & 227.3475 & 1.241 & 0.2305 & \\
\hline & $\mathrm{SC}$ & 5.117 & 3.273 & -1.7604 & 11.9941 & 1.563 & 0.1354 & 1.197 \\
\hline & $\mathrm{AV}$ & -20.768 & 14.156 & -50.5096 & 8.973 & -1.467 & 0.1596 & 1.2113 \\
\hline & T. diag & -0.08 & 0.449 & -1.0237 & 0.8631 & -0.179 & 0.8601 & 1.0528 \\
\hline & $\mathrm{X}: \mathrm{FC}$ & -0.592 & 0.571 & -1.7915 & 0.6079 & -1.036 & 0.3138 & 1.1107 \\
\hline
\end{tabular}

Por cada unidad que aumentó el ángulo de FC de un individuo control con la misma SC, AV, la VL aumentó 0.353 unidades. Y, por cada unidad que aumentó el ángulo de FC de un individuo caso con la misma SC, AV y tiempo de diagnóstico, la VL disminuyó 0.592 unidades. La FC no tuvo un efecto estadísticamente significativo sobre la VL en ninguno de los grupos.

Tabla 30: Bondad de ajuste.

\begin{tabular}{ccc} 
& $\mathbf{R}^{2}$ & p-valor \\
Control & 0.0468 & 0.6264 \\
\hline Caso & 0.3272 & 0.1114 \\
\hline
\end{tabular}

En ninguno de los grupos el modelo de regresión fue estadísticamente significativo. En el grupo caso el modelo explicó el 32,7\% de la variabilidad, y en el grupo control explicó el $4.7 \%$. 


\subsubsection{VL y FCu}

El resultado de la correlación entre la VL y la FCu puede verse en la siguiente tabla (Tabla 31).

Tabla 31: Correlación entre la VL y la FCu.

\begin{tabular}{cccc} 
& & $\begin{array}{c}\text { Rho de } \\
\text { Spearman }\end{array}$ & $\begin{array}{c}\text { p-valor } \\
\text { rho=0 }\end{array}$ \\
\hline \multirow{3}{*}{ FCu } & Control & -0.237 & 0.1409 \\
\cline { 2 - 4 } & Caso & 0.0069 & 0.975 \\
\cline { 2 - 4 } & Total & -0.3278 & $\mathbf{0 . 0 0 8 7}$ \\
\hline
\end{tabular}

En el grupo control la correlación fue negativa, es decir que mayor ángulo de FCu se asoció con menor VL. En el grupo caso la correlación fue prácticamente 0 . En ninguno de los dos casos la relación fue estadísticamente significativa.

El modelo de regresión para la relación entre la $F C u$ y la $V L$ en cada grupo controlando las variables de confusión SC, AV y tiempo de diagnóstico (la última variable sólo está disponible para el grupo caso) y eliminando 2 participantes del grupo control por ser posibles puntos de influencia se puede ver en la Tabla 32.

Tabla 32: Modelo de regresión lineal - VL y FCu.

\begin{tabular}{|c|c|c|c|c|c|c|c|c|}
\hline & & \multirow{2}{*}{$\widehat{\boldsymbol{b}}_{\boldsymbol{i}}$} & \multirow{2}{*}{ EE } & \multicolumn{2}{|c|}{ IC $95 \%$ para $\widehat{b}_{i}$} & \multicolumn{2}{|c|}{$\mathrm{H}_{0}: \widehat{b}_{i}=\mathbf{0}$} & \multirow{2}{*}{ VIF } \\
\hline & & & & Inf. & Sup. & t-valor & p-valor & \\
\hline \multirow{4}{*}{ Control } & (Intercept) & 217.904 & 70.654 & 74.3169 & 361.4909 & 3.084 & 0.004 & \\
\hline & SC & 1.281 & 4.086 & -7.0221 & 9.5849 & 0.314 & 0.7557 & 1.1693 \\
\hline & $\mathrm{AV}$ & 19.182 & 19.42 & -20.2845 & 58.6488 & 0.988 & 0.3303 & 1.1118 \\
\hline & $\mathrm{X}: \mathrm{FCu}$ & -1.683 & 0.705 & -3.116 & -0.2508 & -1.346 & 0.0226 & 1.0752 \\
\hline \multirow{5}{*}{ Caso } & (Intercept) & 17.524 & 46.179 & -79.4932 & 114.5422 & 0.379 & 0.7088 & 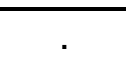 \\
\hline & SC & 6.052 & 3.238 & -0.7513 & 12.8547 & 1.869 & 0.078 & 1.1066 \\
\hline & $\mathrm{AV}$ & -17.107 & 14.192 & -46.9222 & 12.709 & -1.205 & 0.2437 & 1.1502 \\
\hline & T. diag & -0.122 & 0.46 & -1.0891 & 0.8449 & -0.265 & 0.7939 & 1.045 \\
\hline & $\mathrm{X:FCu}$ & 0.057 & 0.403 & -0.7882 & 0.9032 & 0.143 & 0.888 & 1.0106 \\
\hline
\end{tabular}

Por cada unidad que aumentó el ángulo FCu de un individuo control con la misma SC y AV, la VL disminuyó 1.683 unidades. Y, por cada unidad que aumentó el ángulo FCu de un individuo caso con la misma SC, AV y tiempo de diagnóstico, la VL aumentó 0.057 unidades. La FCu tuvo un efecto estadísticamente significativo sobre la VL solamente en el grupo control. 
Tabla 33: Bondad de ajuste.

\begin{tabular}{ccc} 
& $\mathbf{R}^{2}$ & p-valor \\
& & \\
\hline Control & 0.1676 & 0.0968 \\
\hline Caso & 0.2879 & 0.1691 \\
\hline
\end{tabular}

En el grupo control el modelo explicó el $8.2 \%$ de la variabilidad, y en el grupo caso explicó el $28.8 \%$. En ninguno de los dos grupos el modelo fue estadísticamente significativo.

\subsubsection{VL y ACC}

Los datos de la correlación entre la VL y el ACC pueden verse en la Tabla 34.

Tabla 34: Correlación entre la VL y el ACC.

\begin{tabular}{cccc} 
& & $\begin{array}{c}\text { Rho de } \\
\text { Spearman }\end{array}$ & $\begin{array}{c}\text { p-valor } \\
\text { rho=0 }\end{array}$ \\
\hline \multirow{3}{*}{ ACC } & Control & -0.3217 & $\mathbf{0 . 0 4 2 9}$ \\
\cline { 2 - 4 } & Caso & 0.3111 & 0.1485 \\
\cline { 2 - 4 } & Total & -0.0555 & 0.6658 \\
\hline
\end{tabular}

En el grupo control la correlación fue negativa y significativa estadísticamente, es decir que mayor ACC se asoció con menor VL. En el grupo caso la correlación fue positiva, pero no estadísticamente significativa, mayor ACC se asoció con mayor VL.

En la Tabla 35 se muestran los resultados del modelo de regresión usado para averiguar la relación entre el ACC y la VL en cada grupo controlando las variables de confusión SC, AV y tiempo de diagnóstico (la última variable sólo está disponible para el grupo caso). En este análisis dos participantes del grupo control fueron eliminados por ser posibles puntos de influencia. 
Tabla 35: Modelo de regresión lineal - VL y ACC.

\begin{tabular}{|c|c|c|c|c|c|c|c|c|}
\hline & & \multirow{2}{*}{$\widehat{b}_{i}$} & \multirow{2}{*}{ EE } & \multicolumn{2}{|c|}{ IC $95 \%$ para $\widehat{b}_{i}$} & \multicolumn{2}{|c|}{$H_{0}: \widehat{b}_{i}=0$} & \multirow{2}{*}{ VIF } \\
\hline & & & & Inf. & Sup. & t-valor & $p$-valor & \\
\hline \multirow{4}{*}{ Control } & (Intercept) & 298.681 & 116.518 & 61.8867 & 535.4748 & 2.563 & 0.015 & \\
\hline & SC & 0.722 & 4.768 & -8.9669 & 10.4111 & 0.151 & 0.8805 & 1.3516 \\
\hline & $\mathrm{AV}$ & 3.344 & 22.146 & -41.6629 & 48.3501 & 0.151 & 0.8809 & 1.327 \\
\hline & $\mathrm{X}: \mathrm{ACC}$ & -1.091 & 0.503 & -2.1124 & -0.0688 & -2.169 & 0.0372 & 1.2416 \\
\hline \multirow{5}{*}{ Caso } & (Intercept) & -94.564 & 92.715 & -289.3504 & 100.2218 & -1.02 & 0.3213 & \\
\hline & SC & 4.864 & 3.215 & -1.8898 & 11.6173 & 1.513 & 0.1476 & 1.1976 \\
\hline & $\mathrm{AV}$ & -19.054 & 13.563 & -47.5493 & 9.441 & -1.405 & 0.1771 & 1.1537 \\
\hline & T. diag & -0.027 & 0.445 & -0.9618 & 0.9076 & -0.061 & 0.9521 & 1.0721 \\
\hline & $\mathrm{X}: \mathrm{ACC}$ & 0.751 & 0.562 & -0.4287 & 1.9313 & 1.338 & 0.1977 & 1.1021 \\
\hline
\end{tabular}

Por cada unidad que aumentó el ACC de un individuo control con la misma SC y AV, la VL disminuyó 1.091 unidades. Y, por cada unidad que aumentó el ACC de un individuo caso con la misma SC, AV y tiempo de diagnóstico, la VL aumentó 0.751 unidades. El ACC tuvo un efecto estadísticamente significativo sobre la VL solamente en el grupo control

Tabla 36: Bondad de ajuste.

\begin{tabular}{ccc} 
& $\mathbf{R}^{2}$ & p-valor \\
Control & 0.1564 & 0.1184 \\
\hline Caso & 0.3516 & 0.0844 \\
\hline
\end{tabular}

En el grupo control el modelo explicó el $15.6 \%$ de la variabilidad, y en el grupo caso el $35.2 \%$. En ninguno de los dos grupos el modelo fue estadísticamente significativo.

\subsubsection{VL y ACT}

Tras analizar la correlación entre la VL y el ACT, tenemos:

Tabla 37: Correlación entre la VL y el ACT.

\begin{tabular}{cccc} 
& & $\begin{array}{c}\text { Rho de } \\
\text { Spearman }\end{array}$ & $\begin{array}{c}\text { p-valor } \\
\text { rho=0 }\end{array}$ \\
\hline \multirow{3}{*}{ ACT } & Control & 0.2193 & 0.1739 \\
\cline { 2 - 4 } & Caso & -0.024 & 0.9135 \\
\cline { 2 - 4 } & Total & 0.1111 & 0.386 \\
\hline
\end{tabular}


El modelo de regresión para dicha relación en cada grupo controlando las variables de confusión SC, AV y tiempo de diagnóstico (la última variable sólo está disponible para el grupo caso), dio como resultado:

Tabla 38: Modelo de regresión lineal - VL y ACT.

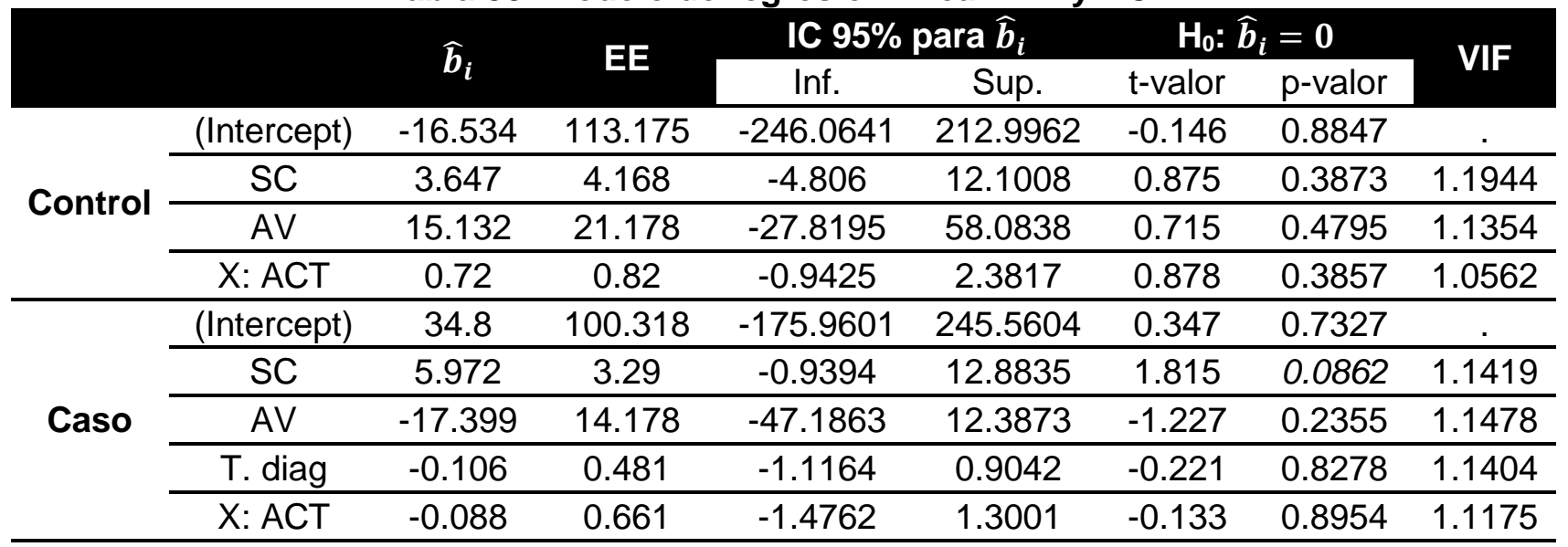

Por cada unidad que aumentó el ACT de un individuo control con la misma SC y AV, la VL aumentó 0.72 unidades. Y, por cada unidad que aumentó el ACT de un individuo caso con la misma SC, AV y tiempo de diagnóstico, la VL disminuyó 0.088 unidades. En ninguno de los dos grupos el ACT tuvo un efecto estadísticamente significativo sobre la VL.

Tabla 39: Bondad de ajuste.

\begin{tabular}{ccc} 
& $\mathbf{R}^{2}$ & p-valor \\
Control & 0.0556 & 0.5541 \\
\hline Caso & 0.2878 & 0.1692 \\
\hline
\end{tabular}

En el grupo control el modelo explicó el 5.6\% de la variabilidad, y en el grupo caso el $28.8 \%$. En ninguno de los dos grupos el modelo fue estadísticamente significativo.

\subsubsection{VL y FT}

La correlación entre la VL y la FT se encuentra en la Tabla 40. 
Tabla 40: Correlación entre la VL y la FT.

\begin{tabular}{cccc} 
& & $\begin{array}{c}\text { Rho de } \\
\text { Spearman }\end{array}$ & $\begin{array}{c}\text { p-valor } \\
\text { rho=0 }\end{array}$ \\
\multirow{3}{*}{ FT } & Control & -0.0598 & 0.7138 \\
\cline { 2 - 4 } & Caso & 0.0845 & 0.7014 \\
\cline { 2 - 4 } & Total & -0.4627 & $\mathbf{0 . 0 0 0 1}$ \\
\hline
\end{tabular}

En el grupo control el mayor ángulo de FT se asoció con menor VL. En el grupo caso un mayor ángulo de FT se asoció con mayor VL. En ninguno de los dos grupos la correlación fue estadísticamente significativa. El modelo de regresión para la relación entre la FT y la VL en cada grupo controlando las variables de confusión SC, AV y tiempo de diagnóstico (la última variable sólo está disponible para el grupo caso) está representado en la Tabla 41.

Tabla 41: Modelo de regresión lineal - VL y FT.

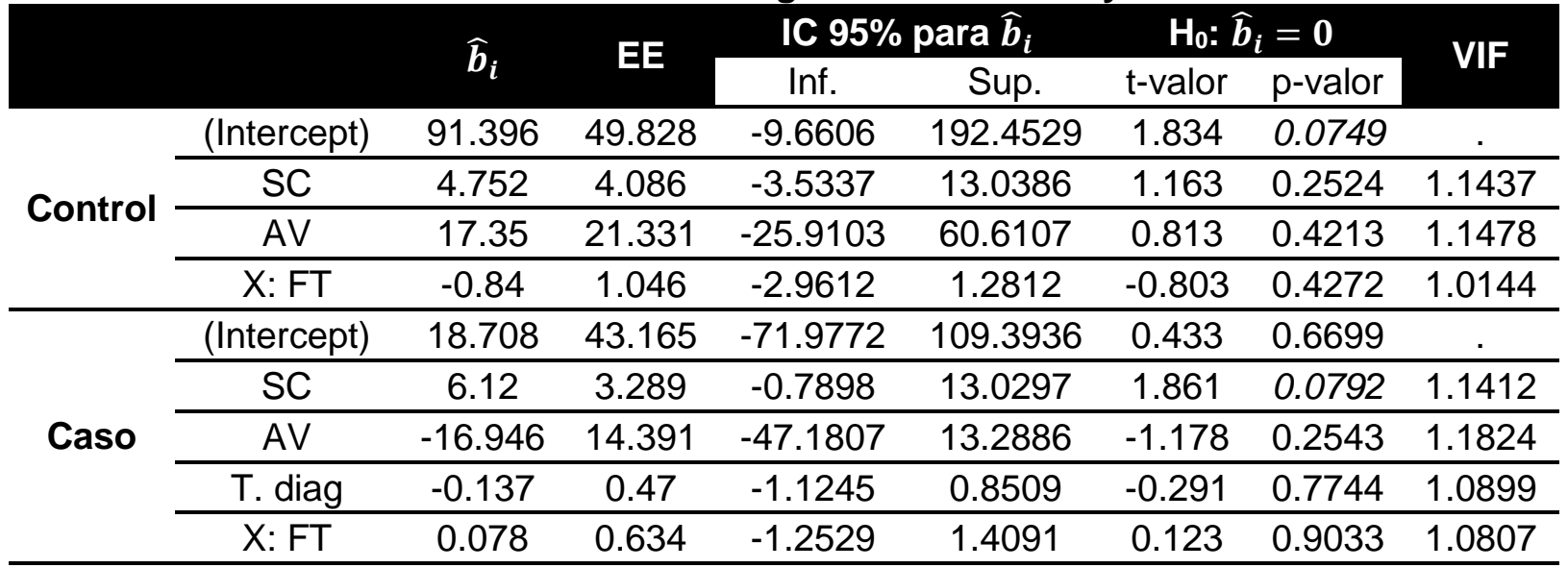

Por cada unidad que aumenta la FT de un individuo control con la misma SC y AV, la VL disminuye 0.84 unidades. Y, por cada unidad que aumenta la FT de un individuo caso con la misma SC, AV y tiempo de diagnóstico, la VL aumenta 0.078 unidades. En ninguno de los dos grupos la FT tuvo un efecto estadísticamente significativo sobre la VL.

Tabla 42: Bondad de ajuste.

\begin{tabular}{ccc} 
& $\mathbf{R}^{2}$ & p-valor \\
Control & 0.0524 & 0.5799 \\
\hline Caso & 0.2877 & 0.1694 \\
\hline
\end{tabular}

En el grupo de control el modelo explicó el 5.2\% de la variabilidad, y en el grupo caso el $28.8 \%$. 
5.7.1.6. VL y AT

La Tabla 43 presenta el resultado de la correlación entre la VL y el AT.

Tabla 43: Correlación entre la VL y el AT.

\begin{tabular}{|c|c|c|c|}
\hline & & $\begin{array}{l}\text { Rho de } \\
\text { Spearman }\end{array}$ & $\begin{array}{c}\text { p-valor } \\
\text { rho }=0\end{array}$ \\
\hline \multirow{3}{*}{ AT } & Control & -0.4324 & 0.0053 \\
\hline & Caso & -0.1058 & 0.631 \\
\hline & Total & -0.0044 & 0.9729 \\
\hline
\end{tabular}

En ambos grupos la correlación fue negativa, es decir que mayor AT se asoció con menor VL, pero sólo fue estadísticamente significativa en el grupo control. Para la relación entre la VL y el AT en cada grupo, controlando las variables de confusión SC, AV y tiempo de diagnóstico (la última variable sólo está disponible para el grupo caso), se realizó el modelo de regresión lineal (Tabla 44).

Tabla 44: Modelo de regresión lineal - VL y AT.

\begin{tabular}{|c|c|c|c|c|c|c|c|c|}
\hline & & \multirow{2}{*}{$\widehat{b}_{i}$} & \multirow{2}{*}{ EE } & \multicolumn{2}{|c|}{ IC $95 \%$ para $\widehat{b}_{i}$} & \multicolumn{2}{|c|}{$\mathrm{H}_{0}: \widehat{b}_{i}=\mathbf{0}$} & \multirow{2}{*}{ VIF } \\
\hline & & & & Inf. & Sup. & t-valor & $\mathrm{p}$-valor & \\
\hline \multirow{4}{*}{ Control } & (Intercept) & 565.674 & 161.858 & 237.4118 & 893.9368 & 3.495 & 0.0013 & \\
\hline & $\mathrm{SC}$ & 2.212 & 3.71 & -5.3128 & 9.736 & 0.596 & 0.5548 & 1.1792 \\
\hline & AV & 23.814 & 19.152 & -15.0267 & 62.6555 & 1.243 & 0.2217 & 1.1569 \\
\hline & $\mathrm{X}: \mathrm{AT}$ & -1.927 & 0.615 & -3.1739 & -0.6797 & -3.133 & 0.0034 & 1.0879 \\
\hline \multirow{5}{*}{ Caso } & (Intercept) & 114.952 & 166.405 & -234.6517 & 464.5553 & 0.691 & 0.4985 & . \\
\hline & SC & 5.853 & 3.23 & -0.9317 & 12.6386 & 1.812 & 0.0866 & 1.1193 \\
\hline & AV & -19.258 & 14.456 & -49.6282 & 11.112 & -1.332 & 0.1994 & 1.2134 \\
\hline & T. diag & -0.128 & 0.456 & -1.0864 & 0.8301 & -0.281 & 0.782 & 1.0434 \\
\hline & X: AT & -0.374 & 0.659 & -1.7579 & 1.009 & -0.569 & 0.5767 & 1.0673 \\
\hline
\end{tabular}

Por cada unidad que aumentó el AT de un individuo control con la misma SC y AV, la VL disminuyó 1.927 unidades. Y, por cada unidad que aumentó el AT de un individuo caso con la misma SC, AV y tiempo de diagnóstico, la VL disminuyó 0.374 unidades. Solamente en el grupo control el AT tuvo un efecto estadísticamente significativo sobre la VL. 
Tabla 45: Bondad de ajuste.

\begin{tabular}{ccc} 
& $\mathbf{R}^{2}$ & p-valor \\
Control & 0.2421 & 0.0176 \\
\hline Caso & 0.2997 & 0.1498 \\
\hline
\end{tabular}

En el grupo control el modelo explicó el $24.2 \%$ de la variabilidad, y en el grupo caso explicó el $30 \%$. En el grupo control el modelo fue estadísticamente significativo.

\subsubsection{VL y AL}

Tras evaluar la correlación entre la VL y el AL, tenemos:

Tabla 46: Correlación entre la VL y el AL.

\begin{tabular}{cccc}
\multirow{4}{*}{ AL } & & $\begin{array}{c}\text { Rho de } \\
\text { Spearman }\end{array}$ & $\begin{array}{c}\text { p-valor } \\
\text { rho=0 }\end{array}$ \\
\cline { 2 - 4 } & Control & -0.3167 & $\mathbf{0 . 0 4 6 5}$ \\
\cline { 2 - 4 } & Caso & -0.372 & 0.0805 \\
\cline { 2 - 4 } & Total & -0.2167 & 0.088 \\
\hline
\end{tabular}

En ambos grupos se asoció un mayor $\mathrm{AL}$ con una menor $\mathrm{VL}$, siendo estadísticamente significativa solamente en el grupo control. Para verificar el efecto de dicho ángulo sobre la VL, se utilizó el modelo e regresión lineal en cada grupo, controlando las variables de confusión SC, AV y tiempo de diagnóstico (la última variable sólo está disponible para el grupo caso) (Tabla 47).

Tabla 47: Modelo de regresión lineal - VL y AL.

\begin{tabular}{|c|c|c|c|c|c|c|c|c|}
\hline & \multirow{2}{*}{$\widehat{\boldsymbol{b}}_{\boldsymbol{i}}$} & \multirow{2}{*}{ EE } & \multicolumn{2}{|c|}{ IC $95 \%$ para $\widehat{b}_{i}$} & \multicolumn{2}{|c|}{$\mathrm{H}_{0}: \widehat{\boldsymbol{b}}_{\boldsymbol{i}}=\mathbf{0}$} & \multirow{2}{*}{ VIF } \\
\hline & & & & Inf. & Sup. & $\mathrm{t}$-valor & p-valor & \\
\hline \multirow{4}{*}{ Control } & (Intercept) & 263.771 & 76.529 & 108.5635 & 418.9787 & 3.447 & 0.0015 & \\
\hline & SC & 6.517 & 3.756 & -1.1011 & 14.1353 & 1.735 & 0.0913 & 1.1758 \\
\hline & AV & 20.193 & 19.297 & -18.9445 & 59.3296 & 1.046 & 0.3024 & 1.1427 \\
\hline & $X: A L$ & -2.142 & 0.732 & -3.626 & -0.6582 & -2.928 & 0.0059 & 1.0364 \\
\hline \multirow{5}{*}{ Caso } & $\overline{\text { (Intercept) }}$ & 120.368 & 70.257 & -27.2368 & 267.972 & 1.713 & 0.1038 & \\
\hline & $\mathrm{SC}$ & 5.672 & 3.052 & -0.7393 & 12.0827 & 1.859 & 0.0795 & 1.1136 \\
\hline & $\mathrm{AV}$ & -17.283 & 13.287 & -45.1986 & 10.6332 & -1.301 & 0.2098 & 1.1425 \\
\hline & T. diag & 0.165 & 0.471 & -0.8232 & 1.1542 & 0.352 & 0.7292 & 1.2378 \\
\hline & $X: A L$ & -1.01 & 0.649 & -2.373 & 0.3539 & -1.556 & 0.1372 & 1.199 \\
\hline
\end{tabular}


Por cada unidad que aumentó el AL de un individuo control con la misma SC y AV, la VL disminuyó 2.142 unidades. Y, por cada unidad que aumentó el $A L$ de un individuo caso con la misma SC, AV y tiempo de diagnóstico, la VL disminuyó 1.01 unidades. Solamente en el grupo control el AL tuvo un efecto estadísticamente significativo sobre la VL.

Tabla 48: Bondad de ajuste.

\begin{tabular}{ccc} 
& $\mathbf{R}^{2}$ & $\mathbf{p}$-valor \\
Control & 0.2209 & $\mathbf{0 . 0 2 7 9}$ \\
\hline Caso & 0.3716 & 0.0664 \\
\hline
\end{tabular}

En el grupo control el modelo explicó el $22.1 \%$ de la variabilidad, y en el grupo caso el $37.2 \%$.

\subsubsection{VL e IP}

Los resultados de la correlación entre la VL y la IP están representados en la Tabla 49.

Tabla 49: Correlación entre la VL y la IP.

\begin{tabular}{cccc} 
& & $\begin{array}{c}\text { Rho de } \\
\text { Spearman }\end{array}$ & $\begin{array}{c}\text { p-valor } \\
\text { rho=0 }\end{array}$ \\
\hline \multirow{3}{*}{ IP } & Control & 0.464 & $\mathbf{0 . 0 0 2 6}$ \\
\cline { 2 - 4 } & Caso & -0.1667 & 0.4584 \\
\cline { 2 - 4 } & Total & -0.1066 & 0.4094 \\
\hline
\end{tabular}

En el grupo control la correlación fue positiva y estadísticamente significativa, es decir que mayor ángulo de IP se asoció con mayor VL. En el grupo caso, un mayor ángulo de IP se asoció con menor VL pero no fue significativo estadísticamente. El modelo de regresión para dicha relación en cada grupo controlando las variables de confusión SC, AV y tiempo de diagnóstico (la última variable sólo está disponible para el grupo caso) está reproducido en la Tabla 50. 
Tabla 50: Modelo de regresión lineal - VL y IP.

\begin{tabular}{|c|c|c|c|c|c|c|c|c|}
\hline & & \multirow{2}{*}{$\widehat{b}_{i}$} & \multirow{2}{*}{ EE } & \multicolumn{2}{|c|}{ IC $95 \%$ para $\widehat{b}_{i}$} & \multicolumn{2}{|c|}{$\mathrm{H}_{0}: \widehat{b}_{i}=\mathbf{0}$} & \multirow{2}{*}{ VIF } \\
\hline & & & & Inf. & Sup. & t-valor & $p$-valor & \\
\hline \multirow{4}{*}{ Control } & (Intercept) & 35.035 & 41.758 & -49.6548 & 119.7244 & 0.839 & 0.407 & \\
\hline & SC & 3.681 & 3.607 & -3.6346 & 10.9971 & 1.021 & 0.3143 & 1.1398 \\
\hline & $\mathrm{AV}$ & 22.49 & 18.876 & -15.7937 & 60.7727 & 1.191 & 0.2413 & 1.1493 \\
\hline & $\mathrm{X}: \mathrm{IP}$ & 2.025 & 0.615 & 0.7785 & 3.2725 & 3.294 & 0.0022 & 1.0252 \\
\hline \multirow{5}{*}{ Caso } & (Intercept) & 25.465 & 36.677 & -51.917 & 102.8468 & 0.694 & 0.4969 & . \\
\hline & SC & 5.787 & 3.239 & -1.0466 & 12.6198 & 1.787 & 0.0918 & 1.0826 \\
\hline & $\mathrm{AV}$ & -12.955 & 14.655 & -43.8734 & 17.9641 & -0.884 & 0.389 & 1.125 \\
\hline & T. diag & -0.143 & 0.459 & -1.1104 & 0.8245 & -0.312 & 0.759 & 1.0446 \\
\hline & $\mathrm{X}: \mathrm{IP}$ & -0.246 & 0.596 & -1.5038 & 1.0117 & -0.413 & 0.685 & 1.0069 \\
\hline
\end{tabular}

Por cada unidad que aumentó la IP de un individuo control con la misma SC y AV, la VL aumentó 2.025 unidades. Y, por cada unidad que aumentó la IP de un individuo caso con la misma SC, AV y tiempo de diagnóstico, la VL disminuyó 0.246 unidades. La IP tuvo un efecto estadísticamente significativo sobre la VL únicamente en el grupo control.

Tabla 51: Bondad de ajuste.

\begin{tabular}{ccc} 
& $\mathbf{R}^{2}$ & p-valor \\
Control & 0.2588 & $\mathbf{0 . 0 1 2 1}$ \\
\hline Caso & 0.2492 & 0.2729 \\
\hline
\end{tabular}

En el grupo control el modelo explicó el $25.9 \%$ de la variabilidad, y en el grupo caso explicó el $24.9 \%$.

\subsubsection{Relación entre la VL y el Equilibrio Corporal}

Si puede ver la correlación entre la VL y el equilibrio en la siguiente tabla (Tabla 52).

Tabla 52: Correlación entre la VL y el equilibrio.

\begin{tabular}{cccc} 
& & $\begin{array}{c}\text { Rho de } \\
\text { Spearman }\end{array}$ & $\begin{array}{c}\text { p-valor } \\
\text { rho=0 }\end{array}$ \\
TUG & Control & -0.2405 & 0.0997 \\
\cline { 2 - 4 } & Caso & 0.1657 & 0.4185 \\
\cline { 2 - 4 } & Total & -0.1898 & 0.1053 \\
\hline
\end{tabular}


En el grupo control se asoció un peor equilibrio con menor VL y en el grupo caso un peor equilibrio se asoció con mayor VL. En ninguno de los dos casos la relación fue estadísticamente significativa. El modelo de regresión lineal fue utilizado para verificar si existió relación entre dichas variables en cada uno de los grupos, controlando las variables de confusión SC, AV y tiempo de diagnóstico (esta última solamente está disponible para el grupo caso) y 4 individuos controles que eran posibles puntos de influencia (Tabla 53).

Tabla 53: Modelo de regresión lineal - VL y equilibrio.

\begin{tabular}{|c|c|c|c|c|c|c|c|c|}
\hline & & \multirow{2}{*}{$\widehat{b}_{i}$} & \multirow{2}{*}{ EE } & \multicolumn{2}{|c|}{ IC $95 \%$ para $\widehat{b}_{i}$} & \multicolumn{2}{|c|}{$\mathrm{H}_{0}: \widehat{\boldsymbol{b}}_{i}=\mathbf{0}$} & \multirow{2}{*}{ VIF } \\
\hline & & & & Inf. & Sup. & t-valor & $\mathrm{p}$-valor & \\
\hline \multirow{4}{*}{ Control } & (Intercept) & 138.849 & 45.652 & 46.5824 & 231.1154 & 3.041 & 0.0041 & \\
\hline & SC & 3.757 & 3.414 & -3.1435 & 10.6584 & 1.1 & 0.2777 & 1.1305 \\
\hline & $\mathrm{AV}$ & 7.395 & 18.913 & -30.8299 & 45.62 & 0.391 & 0.6979 & 1.1491 \\
\hline & X:TUG & -5.217 & 1.967 & -9.1924 & -1.2425 & -2.653 & 0.0114 & 1.0366 \\
\hline \multirow{5}{*}{ Caso } & (Intercept) & 24.708 & 33.412 & -44.7771 & 94.193 & 0.739 & 0.4678 & \\
\hline & SC & 6.025 & 2.994 & -0.2008 & 12.2511 & 2.013 & 0.0572 & 1.1548 \\
\hline & $\mathrm{AV}$ & -17.686 & 13.048 & -44.8202 & 9.4488 & -1.355 & 0.1897 & 1.1564 \\
\hline & T. diag & -0.151 & 0.371 & -0.9221 & 0.6194 & -0.408 & 0.6871 & 1.1522 \\
\hline & X:TUG & -0.112 & 1.549 & -3.3327 & 3.109 & -0.072 & 0.9431 & 1.1571 \\
\hline
\end{tabular}

Por cada unidad que aumentó el valor del TUG de un individuo control con la misma SC y AV, la VL disminuyó 5.217 unidades. Y, por cada unidad que aumentó el valor del TUG de un individuo caso con la misma SC, AV y tiempo de diagnóstico, la VL disminuyó 0.112 unidades. El equilibrio corporal tuvo un efecto estadísticamente significativo sobre la VL solamente en el grupo control.

Tabla 54: Bondad de ajuste.

\begin{tabular}{ccc} 
& $\mathbf{R}^{2}$ & p-valor \\
Control & 0.1884 & $\mathbf{0 . 0 3 7 6}$ \\
\hline Caso & 0.2929 & 0.1071 \\
\hline
\end{tabular}

En el grupo control el modelo explicó el $18.84 \%$ de la variabilidad, y en el grupo caso el $29.3 \%$.

\subsubsection{Relación entre la VL y la Destreza Manual}

La destreza manual fue evaluada a través del Pick Up test en 4 variables: manos derecha e izquierda con los ojos abiertos (MD/OA y MI/OA, respectivamente) y manos derecha e izquierda con los ojo cerrados (MD/OC y $\mathrm{MI} / \mathrm{OC})$. Sin embargo, teniendo en cuenta que casi todos los participantes eran 
diestros (excepto 1) y el objetivo era conocer su relación con la VL, se analizó solamente la destreza manual con la mano derecha y ojos abiertos.

La correlación entre la VL y la destreza manual está reflejada en la siguiente tabla (Tabla 55).

Tabla 55: Correlación entre la VL y la destreza manual.

\begin{tabular}{c|ccc} 
& & $\begin{array}{c}\text { Rho de } \\
\text { Spearman }\end{array}$ & $\begin{array}{c}\text { p-valor } \\
\text { rho=0 }\end{array}$ \\
\hline \multirow{3}{*}{ MD/OA A } & Control & -0.1166 & 0.4302 \\
\cline { 2 - 4 } & Caso & -0.2925 & 0.1471 \\
\cline { 2 - 4 } & Total & -0.497 & $\mathbf{< 0 . 0 0 0 1}$ \\
\hline
\end{tabular}

En los dos grupos una peor destreza manual se asoció con una menor VL. Sin embargo, dicha correlación no fue estadísticamente significativa en ninguno de los dos grupos. Se utilizó el modelo de regresión lineal en cada uno de los grupos, controlando las variables de confusión SC, AV y tiempo de diagnóstico (esta última solamente está disponible para el grupo caso) (Tabla 56).

Tabla 56: Modelo de regresión lineal - VL y destreza manual.

\begin{tabular}{|c|c|c|c|c|c|c|c|c|}
\hline & & \multirow{2}{*}{$\widehat{b}_{i}$} & \multirow{2}{*}{ EE } & \multicolumn{2}{|c|}{ IC $95 \%$ para $\widehat{b}_{i}$} & \multicolumn{2}{|c|}{$H_{0}: \widehat{b}_{i}=\mathbf{0}$} & \multirow{2}{*}{ VIF } \\
\hline & & & & Inf. & Sup. & t-valor & $p$-valor & \\
\hline \multirow{4}{*}{ Control } & (Intercept) & 79.212 & 59.926 & -41.5618 & 199.986 & 1.322 & 0.1931 & \\
\hline & SC & 5.103 & 4.096 & -3.1531 & 13.3586 & 1.246 & 0.2195 & 1.1833 \\
\hline & AV & 16.736 & 21.66 & -26.9164 & 60.3876 & 0.773 & 0.4439 & 1.1304 \\
\hline & $\mathrm{X}: \mathrm{MD} / \mathrm{OA}$ & -1.082 & 2.524 & -6.1692 & 4.0046 & -0.429 & 0.6702 & 1.064 \\
\hline \multirow{5}{*}{ Caso } & (Intercept) & 50.878 & 48.108 & -49.1682 & 150.9243 & 1.058 & 0.3023 & \\
\hline & SC & 5.194 & 3.136 & -1.3284 & 11.7159 & 1.656 & 0.1126 & 1.2977 \\
\hline & $\mathrm{AV}$ & -17.781 & 12.772 & -44.3407 & 8.7795 & -1.392 & 0.1784 & 1.1346 \\
\hline & T. diag & -0.006 & 0.41 & -0.86 & 0.847 & -0.016 & 0.9876 & 1.4469 \\
\hline & $\mathrm{X}: \mathrm{MD} / \mathrm{OA}$ & -1.634 & 2.288 & -6.392 & 3.1246 & -0.714 & 0.4831 & 1.4823 \\
\hline
\end{tabular}

Por cada unidad que aumenta el Pick Up test de un individuo control con la misma SC y AV, la VL disminuye 1.082 unidades. $Y$, por cada unidad que aumenta el Pick Up test de un individuo caso con la misma SC, AV y tiempo de diagnóstico, la VL disminuye 1.634 unidades. En ninguno de los dos grupos la destreza manual tuvo un efecto estadísticamente significativo sobre la VL. 
Tabla 57: Bondad de ajuste.

\begin{tabular}{ccc} 
& $\mathbf{R}^{2}$ & $\mathbf{p}$-valor \\
Control & 0.0493 & 0.522 \\
\hline Caso & 0.3095 & 0.0871 \\
\hline
\end{tabular}

En el grupo control el modelo explicó el $4.9 \%$ de la variabilidad, y en el grupo caso el $31 \%$.

\subsubsection{Relación entre la VL y la Función Visual}

En este estudio, la función visual fue evaluada según dos variables: SC y AV. La correlación entre ambas y la VL se ve reflejada en la Tabla 58.

\begin{tabular}{|c|c|c|c|}
\hline & & $\begin{array}{l}\text { Rho de } \\
\text { Spearman }\end{array}$ & $\begin{array}{c}\text { p-valor } \\
\text { rho }=0\end{array}$ \\
\hline \multirow{3}{*}{$\begin{array}{c}\text { SC } \\
\text { Binocular }\end{array}$} & Control & 0.2851 & 0.0495 \\
\hline & Caso & 0.5731 & 0.0022 \\
\hline & Total & 0.6608 & $<0.0001$ \\
\hline \multirow{3}{*}{ AV } & Control & -0.0384 & 0.7954 \\
\hline & Caso & -0.4187 & 0.0333 \\
\hline & Total & -0.595 & $<0.0001$ \\
\hline
\end{tabular}

Para SC, la correlación fue estadísticamente significativa y positiva en los dos grupos, es decir que mayor SC se asoció con mayor VL. Para la AV la correlación fue negativa y sólo estadísticamente significativa en el grupo caso, peor AV se asoció con menor VL. El modelo de regresión en cada grupo controlando las variables de confusión SC, AV y tiempo de diagnóstico (esta última solamente está disponible para el grupo caso) dio lo siguientes resultados:

Tabla 59: Modelo de regresión lineal - VL y Función visual.

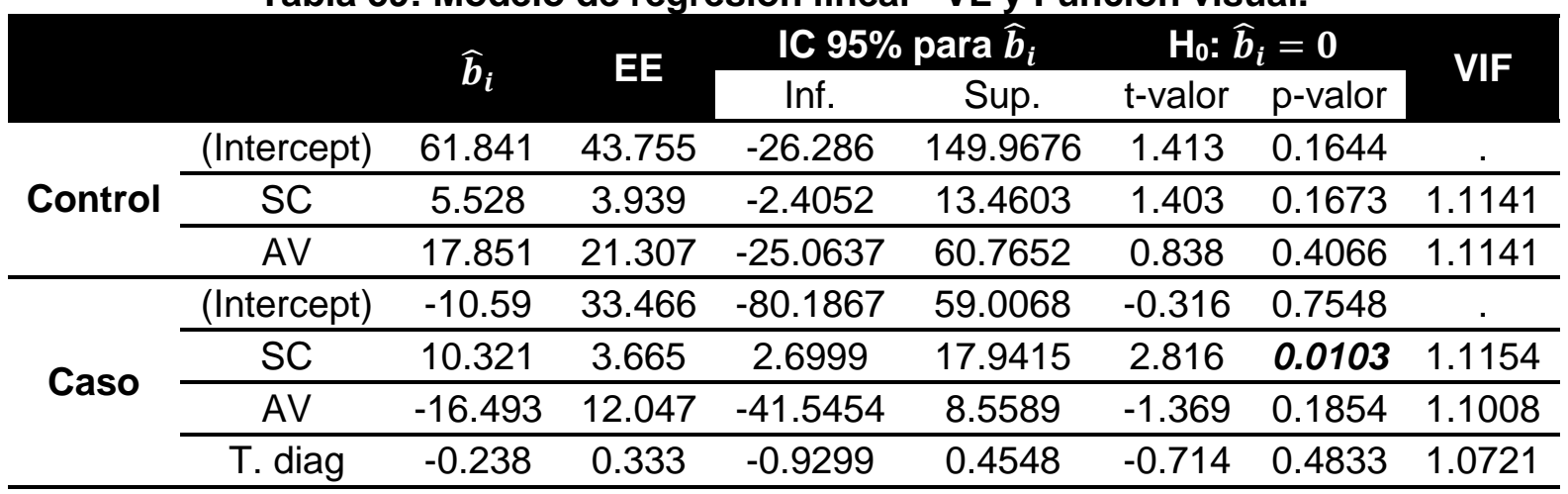


Por cada unidad que aumentó la SC binocular de un individuo control con la misma AV, la VL aumentó 5.528 unidades. $Y$, por cada unidad que aumentó la AV de un individuo control con la misma SC, la VL aumentó 17.851 unidades. Para el grupo caso, por cada unidad que aumentó la SC de un individuo caso con la misma AV y tiempo de diagnóstico, la VL aumentó 5.995 unidades. En este caso el coeficiente se quedó al borde de la significación estadística. Y, por cada unidad que aumentó la AV de un individuo caso con la misma SC y tiempo de diagnóstico, la VL disminuyó 17.554 unidades. Para el grupo control, la función visual no tuvo un efecto estadísticamente significativo sobre la VL y, para el grupo caso solamente el efecto de la SC sobre la VL fue significativo estadísticamente.

Tabla 60: Bondad de ajuste.

\begin{tabular}{ccc} 
& $\mathbf{R}^{2}$ & p-valor \\
Control & 0.0454 & 0.3519 \\
\hline Caso & 0.2927 & 0.0507 \\
\hline
\end{tabular}

En el grupo control el modelo explicó el $4.5 \%$ de la variabilidad y en el grupo caso el modelo explicó el $29.3 \%$ de la variabilidad.

\subsubsection{Relación entre la VL y la Depresión}

La siguiente tabla presenta el resultado del análisis de correlación entre la VL y la sintomatología depresiva (Tabla 61).

Tabla 61: Correlación entre la VL y la sintomatología depresiva.

\begin{tabular}{cccc}
\hline \multirow{4}{*}{ GDS } & & $\begin{array}{c}\text { Rho de } \\
\text { Spearman }\end{array}$ & $\begin{array}{c}\text { p-valor } \\
\text { rho=0 }\end{array}$ \\
\cline { 2 - 4 } & Control & -0.1564 & 0.2884 \\
\cline { 2 - 4 } & Caso & -0.3144 & 0.1178 \\
\cline { 2 - 4 } & Total & -0.3436 & $\mathbf{0 . 0 0 2 7}$ \\
\hline
\end{tabular}

En ambos grupos la correlación fue negativa, es decir que mayor puntuación en GDS se asoció con menor VL. En ninguno de los dos casos la relación fue estadísticamente significativa. El modelo de regresión lineal controlando las variables de confusión SC, AV y tiempo de diagnóstico (esta última solamente está disponible para el grupo caso) está reflejado en la Tabla 62. 
Tabla 62: Modelo de regresión lineal - VL y GDS.

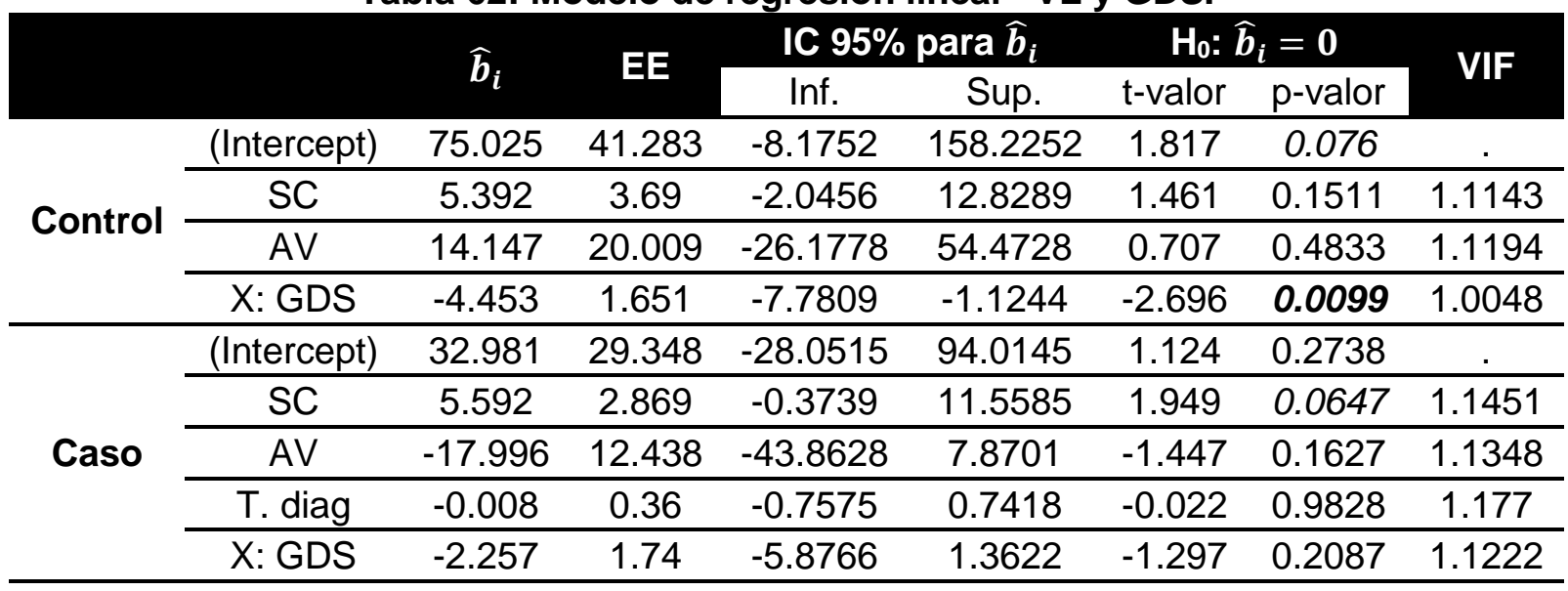

Por cada unidad que aumentó la sintomatología depresiva de un individuo control con la misma SC y AV, la VL disminuyó 4.453 unidades. Y, por cada unidad que aumentó la sintomatología depresiva de un individuo caso con la misma SC, AV y tiempo de diagnóstico, la VL disminuyó 2.257 unidades. La sintomatología depresiva tuvo un efecto significativo estadísticamente sobre la $\mathrm{VL}$ solamente en el grupo control.

Tabla 63: Bondad de ajuste.

\begin{tabular}{ccc} 
& $\mathbf{R}^{2}$ & $\mathbf{p}$-valor \\
Control & 0.1807 & $\mathbf{0 . 0 3 1 1}$ \\
\hline Caso & 0.3452 & 0.0543 \\
\hline
\end{tabular}

En el grupo control el modelo explicó el $18.1 \%$ de la variabilidad, y en el grupo caso el $34.5 \%$.

\subsubsection{Relación entre la Depresión y la Postura Corporal}

Se consideró la variable depresión en dos grupos, G1) No sintomatología depresiva: GDS $\leq 5$ y G2) Posible sintomatología depresiva: GDS $>5$. Las hipótesis de normalidad y homogeneidad de varianzas fueron verificadas con los contrastes SW y BF, respectivamente (Tabla 64). 
Tabla 64: Datos descriptivos y contrastes de normalidad y homogeneidad de varianzas para la relación entre la depresión y la postura en sedestación.

\begin{tabular}{|c|c|c|c|c|c|c|c|c|c|}
\hline \multirow{2}{*}{\multicolumn{2}{|c|}{$\begin{array}{c}\text { Sintomatología } \\
\text { Depresiva }\end{array}$}} & \multirow[t]{2}{*}{$\mathbf{N}$} & \multirow[t]{2}{*}{ Media } & \multirow[t]{2}{*}{ DT } & \multirow[t]{2}{*}{ Mediana } & \multicolumn{2}{|c|}{$\begin{array}{c}\mathrm{H}_{0}: \\
\text { Normalidad }\end{array}$} & \multicolumn{2}{|c|}{$\begin{array}{c}\mathrm{H}_{0}: \\
\text { Homogeneidad } \\
\text { de varianzas } \\
\text { (BF) }\end{array}$} \\
\hline & & & & & & SW & $p$-valor & $\begin{array}{c}\mathrm{F} \\
\text { valor }\end{array}$ & $p$-valor \\
\hline \multirow{3}{*}{ FC } & No & 53 & 78.97 & 13.76 & 81.4 & 0.979 & 0.4861 & \multirow{3}{*}{0.03} & \multirow{3}{*}{0.8702} \\
\hline & Posible & 11 & 81.1 & 12.62 & 81.4 & 0.903 & 0.1989 & & \\
\hline & Total & 64 & 79.34 & 13.5 & 81.4 & 0.977 & 0.2657 & & \\
\hline \multirow{3}{*}{$\mathrm{FCu}$} & No & 53 & 69.81 & 12.71 & 67.4 & 0.957 & 0.0546 & \multirow{3}{*}{0.35} & \multirow{3}{*}{0.5574} \\
\hline & Posible & 11 & 66.44 & 10.99 & 67.1 & 0.909 & 0.235 & & \\
\hline & Total & 64 & 69.23 & 12.42 & 67.25 & 0.956 & 0.0222 & & \\
\hline \multirow{3}{*}{ ACC } & No & 53 & 169.8 & 12.18 & 167.3 & 0.97 & 0.2081 & \multirow{3}{*}{0.21} & \multirow{3}{*}{0.6467} \\
\hline & Posible & 11 & 163.59 & 13.72 & 167.8 & 0.957 & 0.7284 & & \\
\hline & Total & 64 & 168.73 & 12.57 & 167.55 & 0.988 & 0.7736 & & \\
\hline \multirow{3}{*}{ ACT } & No & 53 & 137.74 & 7.6 & 136.7 & 0.992 & 0.9828 & \multirow{3}{*}{0.64} & \multirow{3}{*}{0.4284} \\
\hline & Posible & 11 & 143.86 & 8.89 & 142.8 & 0.966 & 0.8405 & & \\
\hline & Total & 64 & 138.79 & 8.1 & 137.8 & 0.992 & 0.9542 & & \\
\hline \multirow{3}{*}{$\mathbf{F T}$} & No & 53 & 27.67 & 9.63 & 25.1 & 0.928 & 0.0033 & \multirow{3}{*}{0.83} & \multirow{3}{*}{0.3661} \\
\hline & Posible & 11 & 30.61 & 6.5 & 28.9 & 0.927 & 0.3848 & & \\
\hline & Total & 64 & 28.18 & 9.19 & 25.6 & 0.946 & 0.0075 & & \\
\hline \multirow{3}{*}{ AT } & No & 53 & 242.1 & 8.56 & 242.4 & 0.981 & 0.5698 & \multirow{3}{*}{2.01} & \multirow{3}{*}{0.1612} \\
\hline & Posible & 11 & 238.01 & 11.91 & 235.5 & 0.927 & 0.3828 & & \\
\hline & Total & 64 & 241.4 & 9.24 & 241.7 & 0.982 & 0.4731 & & \\
\hline \multirow{3}{*}{$A L$} & No & 53 & 98.55 & 7.09 & 99.6 & 0.968 & 0.1659 & \multirow{3}{*}{7.83} & \multirow{3}{*}{0.0068} \\
\hline & Posible & 11 & 101.55 & 11.97 & 100.6 & 0.964 & 0.8161 & & \\
\hline & Total & 64 & 99.07 & 8.1 & 99.65 & 0.991 & 0.9172 & & \\
\hline \multirow{3}{*}{ IP } & No & 52 & 25.69 & 9.14 & 25.75 & 0.973 & 0.2809 & \multirow{3}{*}{1.16} & \multirow{3}{*}{0.286} \\
\hline & Posible & 11 & 23.55 & 11.88 & 24.5 & 0.942 & 0.5415 & & \\
\hline & Total & 63 & 25.32 & 9.6 & 25.7 & 0.977 & 0.2755 & & \\
\hline
\end{tabular}

Se rechazó la normalidad solamente para el ángulo de FT, con lo cual se utilizó el t-Student para evaluar la igualdad de medias en todos los otros ángulos y el test $U$ de Mann-Whitney para la igualdad de medianas para la FT (Tabla 65). 
Tabla 65: Igualdad de medias para la relación entre la depresión y la postura en sedestación.

\begin{tabular}{ccc}
\hline $\begin{array}{c}\text { Ángulos } \\
\text { posturales }\end{array}$ & \multicolumn{2}{c}{$\begin{array}{c}\mathbf{H}_{0} \text { : igualdad de medias } \\
\text { t-Student }\end{array}$} \\
& t-test & $\mathrm{p}$-valor \\
\hline FC & -0.473 & 0.6382 \\
\hline FCu & 0.818 & 0.4164 \\
\hline ACC & 1.505 & 0.1373 \\
\hline ACT & -2.364 & $\mathbf{0 . 0 2 1 2}$ \\
\hline FT $\left(^{*}\right)$ & 205.5 & 0.1281 \\
\hline AT & 1.344 & 0.1838 \\
\hline AL & -0.8 & 0.44 \\
\hline IP & 0.671 & 0.5047 \\
\hline
\end{tabular}

$\left.{ }^{*}\right)$ Test U de Mann-Whitney

El ACT fue significativamente mayor en el grupo de posibles deprimidos, siendo esta diferencia estadísticamente significativa. Para saber cómo la postura influyó en la posibilidad de estar deprimido se utilizó el modelo de regresión logística controlando el factor de confusión grupo de estudio (Tabla 66). 
Tabla 66: Modelo de regresión logística - Depresión y Postura en sedestación.

\begin{tabular}{|c|c|c|c|c|c|c|c|}
\hline & & & & & 0 & IC 9 & $\%$ OR \\
\hline & Estimacion & estándar & L-valor & valor & U. & Inferior & Superior \\
\hline (Intercept) & -0.8534 & 2.2147 & -0.3853 & 0.7 & & & \\
\hline FC & -0.0184 & 0.0299 & -0.6156 & 0.5382 & 0.9818 & 0.9259 & 1.041 \\
\hline $\begin{array}{c}\text { Grupo } \\
\text { Caso }\end{array}$ & 1.5764 & 0.8287 & 1.9023 & 0.0571 & 4.8377 & 0.9533 & 24.5494 \\
\hline (Intercept) & 0.8979 & 1.949 & 0.4607 & 0.645 & & . & . \\
\hline $\mathrm{FCu}$ & -0.0484 & 0.0299 & -1.6197 & 0.1053 & 0.9528 & 0.8986 & 1.0102 \\
\hline $\begin{array}{c}\text { Grupo } \\
\text { Caso }\end{array}$ & 1.7796 & 0.7572 & 2.3504 & 0.0188 & 5.9278 & 1.344 & 26.1449 \\
\hline (Intercept) & 5.2047 & 5.2448 & 0.9924 & 0.321 & & & \\
\hline ACC & -0.0444 & 0.0318 & -1.3961 & 0.1627 & 0.9566 & 0.8988 & 1.0181 \\
\hline $\begin{array}{c}\text { Grupo } \\
\text { Caso }\end{array}$ & 1.2896 & 0.7059 & 1.8269 & 0.0677 & 3.6314 & 0.9103 & 14.4861 \\
\hline (Intercept) & -15.7107 & 6.5326 & -2.405 & 0.0162 & . & . & . \\
\hline ACT & 0.0962 & 0.0458 & 2.0997 & 0.0358 & 1.1009 & 1.0064 & 1.2043 \\
\hline $\begin{array}{c}\text { Grupo } \\
\text { Caso }\end{array}$ & 1.3217 & 0.7264 & 1.8195 & 0.0688 & 3.7497 & 0.903 & 15.5702 \\
\hline (Intercept) & -2.0211 & 1.1068 & -1.8261 & 0.0678 & . & . & . \\
\hline FT & -0.0073 & 0.0406 & -0.1804 & 0.8569 & 0.9927 & 0.9167 & 1.075 \\
\hline $\begin{array}{c}\text { Grupo } \\
\text { Caso }\end{array}$ & 1.3889 & 0.8178 & 1.6983 & 0.0895 & 4.0104 & 0.8073 & 19.9214 \\
\hline (Intercept) & 5.4059 & 9.3535 & 0.578 & 0.5633 & & & \\
\hline AT & -0.0313 & 0.0386 & -0.8112 & 0.4172 & 0.9691 & 0.8985 & 1.0454 \\
\hline $\begin{array}{c}\text { Grupo } \\
\text { Caso } \\
\end{array}$ & 1.147 & 0.7214 & 1.5899 & 0.1119 & 3.1488 & 0.7657 & 12.9486 \\
\hline (Intercept) & -6.4888 & 4.2213 & -1.5372 & 0.1242 & . & . & . \\
\hline$A L$ & 0.043 & 0.0417 & 1.0327 & 0.3017 & 1.044 & 0.9621 & 1.1328 \\
\hline $\begin{array}{c}\text { Grupo } \\
\text { Caso }\end{array}$ & 1.2953 & 0.6991 & 1.8529 & 0.0639 & 3.6521 & 0.9279 & 14.3742 \\
\hline (Intercept) & -0.9819 & 0.9687 & -1.0137 & 0.3107 &. & $\cdot$ & $\cdot$ \\
\hline IP & -0.0567 & 0.0403 & -1.4063 & 0.1596 & 0.9449 & 0.8731 & 1.0226 \\
\hline $\begin{array}{c}\text { Grupo } \\
\text { Caso }\end{array}$ & 1.7688 & 0.7676 & 2.3043 & 0.0212 & 5.8635 & 1.3025 & 26.3952 \\
\hline
\end{tabular}

De todos los ángulos evaluados en sedestación, el ACT fue el único que tuvo un efecto estadísticamente significativo sobre la probabilidad de tener sintomatología depresiva: la probabilidad de presentar sintomatología depresiva fue 1.1 veces mayor cuando este ángulo aumentó una unidad.

\subsubsection{Relación entre la Depresión y el Equilibrio Corporal}

Las hipótesis de normalidad y de homogeneidad de varianzas fueron evaluadas con los contrastes de SW y BF, respectivamente (Tabla 67). 
Tabla 67: Datos descriptivos y contrastes de normalidad y homogeneidad de varianzas para la relación entre la depresión y el equilibrio.

\begin{tabular}{|c|c|c|c|c|c|c|c|c|}
\hline \multirow[t]{2}{*}{$\begin{array}{c}\text { Sintomatología } \\
\text { depresiva }\end{array}$} & \multirow[t]{2}{*}{$\mathbf{N}$} & \multirow[t]{2}{*}{ Media } & \multirow[t]{2}{*}{ DT } & \multirow[t]{2}{*}{ Mediana } & \multicolumn{2}{|c|}{$\begin{array}{c}\mathrm{H}_{0}: \\
\text { Normalidad }\end{array}$} & \multicolumn{2}{|c|}{$\begin{array}{c}\text { Ho: } \\
\text { Homogeneidad } \\
\text { de varianzas } \\
(\mathrm{BF})\end{array}$} \\
\hline & & & & & SW & $\mathrm{p}$-valor & $\mathrm{F}$ valor & $\mathrm{p}$-valor \\
\hline No & 65 & 10.66 & 3 & 10 & 0.897 & 0.0001 & \multirow{3}{*}{0.4} & \multirow{3}{*}{0.5284} \\
\hline Posible & 15 & 13.53 & 3.16 & 14 & 0.949 & 0.5125 & & \\
\hline Total & 80 & 11.2 & 3.22 & 10 & 0.918 & 0.0001 & & \\
\hline
\end{tabular}

No se rechazó la hipótesis de homogeneidad de varianzas sin embargo sí la de normalidad, con lo cual se utilizó el contraste $U$ de Mann-Whitney para evaluar la igualdad de medianas entre los grupos (Tabla 68).

Tabla 68: Igualdad de medianas para la relación entre la depresión y el equilibrio.

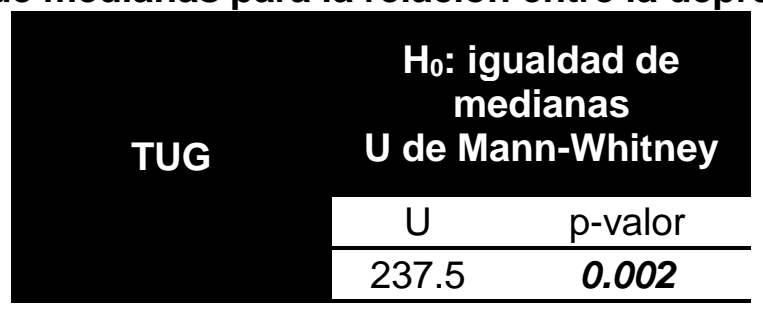

La igualdad de medianas fue significativa estadísticamente, siendo el valor de la variable TUG significativamente mayor en el grupo con más sintomatología depresiva, es decir el grupo de deprimidos tuvo un peor equilibrio. Y para evaluar la influencia del equilibrio sobre la posibilidad de presentar o no sintomatología depresiva se utilizó el modelo de regresión logística controlando el grupo como factor de confusión (Tabla 69).

Tabla 69: Modelo de regresión logística - Depresión y Equilibrio.

\begin{tabular}{cccccccc} 
& Estimación & $\begin{array}{c}\text { Error } \\
\text { estándar }\end{array}$ & Z-valor & $\begin{array}{c}\text { p- } \\
\text { valor }\end{array}$ & O.R. & \multicolumn{2}{c}{ IC 95\% OR } \\
(Intercept) & -4.8306 & 1.2325 & -3.9192 & $\mathbf{0 . 0 0 0 1}$ &. &. &. \\
\hline TUG & 0.2475 & 0.0931 & 2.6596 & $\mathbf{0 . 0 0 7 8}$ & 1.2808 & 1.0673 & 1.537 \\
\hline $\begin{array}{c}\text { Grupo } \\
\text { Caso }\end{array}$ & 0.8636 & 0.6263 & 1.3789 & 0.1679 & 2.3716 & 0.6949 & 8.0934 \\
\hline
\end{tabular}

El equilibrio corporal tuvo una asociación significativa estadísticamente con tener o no sintomatología depresiva. Aumentar el valor del TUG fue un factor de riesgo. En general, la probabilidad de presentar sintomatología depresiva fue 1.28 veces mayor cuando el valor del TUG aumentó en un punto. 
5.7.8. Relación entre la Depresión y la Destreza Manual

Se comprobó, en primer lugar, la hipótesis de normalidad y la de homogeneidad de varianzas con los contrastes de SW y BF, respectivamente (Tabla 70).

Tabla 70: Datos descriptivos y contrastes de normalidad y homogeneidad de varianzas para la relación entre la depresión y la destreza manual.

\begin{tabular}{|c|c|c|c|c|c|c|c|c|c|}
\hline \multirow{2}{*}{\multicolumn{2}{|c|}{$\begin{array}{c}\text { Sintomatología } \\
\text { depresiva }\end{array}$}} & \multirow[t]{2}{*}{$\mathbf{N}$} & \multirow[t]{2}{*}{ Media } & \multirow[t]{2}{*}{ DT } & \multirow[t]{2}{*}{ Mediana } & \multicolumn{2}{|c|}{$\begin{array}{c}\mathrm{H}_{0}: \\
\text { Normalidad }\end{array}$} & \multicolumn{2}{|c|}{$\begin{array}{c}\mathrm{H}_{0}: \\
\text { Homogeneidad } \\
\text { de varianzas } \\
\text { (BF) }\end{array}$} \\
\hline & & & & & & SW & $\mathrm{p}$-valor & $\mathrm{F}$ valor & $p$-valor \\
\hline \multirow{3}{*}{ MD/OA } & No & 65 & 12.29 & 2.45 & 12 & 0.871 & $<0.0001$ & \multirow{3}{*}{1.99} & \multirow{3}{*}{0.1623} \\
\hline & Posible & 15 & 13.93 & 3.43 & 14 & 0.938 & 0.3586 & & \\
\hline & Total & 80 & 12.6 & 2.72 & 12 & 0.886 & $<0.0001$ & & \\
\hline \multirow{3}{*}{ MD/OC } & No & 65 & 25.46 & 7.8 & 24 & 0.829 & $<0.0001$ & \multirow{3}{*}{0} & \multirow{3}{*}{0.9528} \\
\hline & Posible & 15 & 28.47 & 7.63 & 27 & 0.931 & 0.2794 & & \\
\hline & Total & 80 & 26.02 & 7.81 & 26 & 0.864 & $<0.0001$ & & \\
\hline
\end{tabular}

No se rechazó la hipótesis de homogeneidad de varianzas, pero sí la de normalidad, con lo cual se utilizó el contraste $U$ de Mann-Whitney para evaluar la igualdad de medianas entre los dos grupos (Tabla 71).

Tabla 71: Igualdad de medianas para la relación entre la depresión y la destreza manual.

\begin{tabular}{|c|c|c|}
\hline \multirow[t]{2}{*}{$\begin{array}{c}\text { Pick Up } \\
\text { test }\end{array}$} & \multicolumn{2}{|c|}{$\begin{array}{c}\mathrm{H}_{0} \text { : igualdad de } \\
\text { medianas } \\
\text { U de Mann-Whitney }\end{array}$} \\
\hline & $U$ & $\mathrm{p}$-valor \\
\hline MD/OA & 322.5 & 0.0402 \\
\hline MD/OC & 357 & 0.1084 \\
\hline
\end{tabular}

Se rechazó la hipótesis de igualdad de medianas en la MD con OA siendo la diferencia entre los dos grupos estadísticamente significativa, es decir la destreza manual fue significativamente peor en el grupo con más sintomatología depresiva. En la Tabla 72 es posible ver el modelo de regresión logística controlando el factor de confusión grupo de estudio. 
Tabla 72: Modelo de regresión logística - Depresión y Destreza manual.

\begin{tabular}{cccccccc} 
& Estimación & $\begin{array}{c}\text { Error } \\
\text { estándar }\end{array}$ & Z-valor & $\begin{array}{c}\text { p- } \\
\text { valor }\end{array}$ & O.R. & \multicolumn{2}{c}{ IC 95\% OR } \\
(Intercept) & -3.7528 & 1.3871 & -2.7056 & $\mathbf{0 . 0 0 6 8}$ &. &. & Superior \\
\hline MD/OA & 0.1494 & 0.1079 & 1.3852 & 0.166 & 1.1612 & 0.9399 & 1.4345 \\
\hline $\begin{array}{c}\text { Grupo } \\
\text { Caso }\end{array}$ & 0.7226 & 0.6465 & 1.1176 & 0.2637 & 2.0597 & 0.5801 & 7.3133 \\
\hline (Intercept) & -3.2479 & 1.0442 & -3.1103 & $\mathbf{0 . 0 0 1 9}$ &. &. &. \\
\hline MD/OC & 0.0468 & 0.0336 & 1.3952 & 0.1629 & 1.0479 & 0.9812 & 1.1192 \\
\hline $\begin{array}{c}\text { Grupo } \\
\text { Caso }\end{array}$ & 1.1322 & 0.5998 & 1.8876 & 0.0591 & 3.1024 & 0.9576 & 10.0515 \\
\hline
\end{tabular}

Ninguna de las variables relacionadas con el pick up test tuvo un efecto estadísticamente significativo sobre la probabilidad de tener sintomatología depresiva cuando se considera el grupo como factor de confusión.

\subsubsection{Relación entre la Depresión y el Miedo a Caerse}

Las hipótesis de normalidad y homogeneidad de varianzas fueron comprobadas con los contrastes de SW y BF, respectivamente (Tabla 73).

Tabla 73: Datos descriptivos y contrastes de normalidad y homogeneidad de varianzas para la relación entre la depresión y el miedo a caerse.

\begin{tabular}{|c|c|c|c|c|c|c|c|c|}
\hline \multirow[t]{2}{*}{$\begin{array}{c}\text { Sintomatología } \\
\text { depresiva }\end{array}$} & \multirow[t]{2}{*}{$\mathbf{N}$} & \multirow[t]{2}{*}{ Media } & \multirow[t]{2}{*}{ DT } & \multirow[t]{2}{*}{ Mediana } & \multicolumn{2}{|c|}{$\begin{array}{c}\mathrm{H}_{0}: \\
\text { Normalidad }\end{array}$} & \multicolumn{2}{|c|}{$\begin{array}{c}\mathrm{H}_{0}: \\
\text { Homogeneidad } \\
\text { de varianzas } \\
\text { (BF) }\end{array}$} \\
\hline & & & & & SW & $p$-valor & $\begin{array}{c}\mathrm{F} \\
\text { valor }\end{array}$ & $\mathrm{p}$-valor \\
\hline No & 65 & 20.57 & 5.38 & 19 & 0.724 & $<0.0001$ & \multirow{3}{*}{0.34} & \multirow{3}{*}{0.5637} \\
\hline Posible & 15 & 22.07 & 3.26 & 22 & 0.987 & 0.9973 & & \\
\hline Total & 80 & 20.85 & 5.07 & 20 & 0.772 & $<0.0001$ & & \\
\hline
\end{tabular}

La hipótesis de normalidad fue rechazada, con lo cual se utilizó el contraste $U$ de Mann-Whitney para evaluar la igualdad de medianas (Tabla 74).

Tabla 74: Igualdad de medianas para la relación entre la depresión y el miedo a caerse.

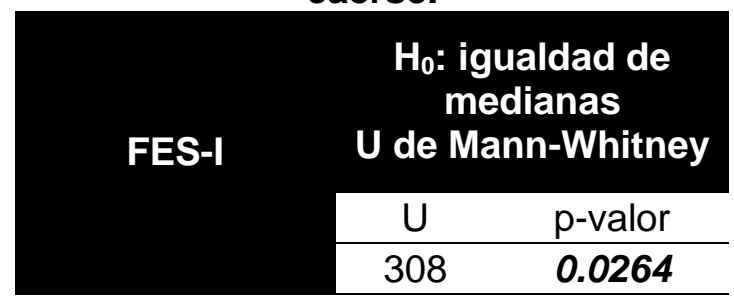


El miedo a caerse fue estadísticamente significativo, siendo mayor en el grupo con más sintomatología depresiva. Para evaluar cómo el miedo a caerse afecta a la posibilidad de presentar sintomatología depresiva o no se utilizó el modelo de regresión logística controlando el grupo como factor de confusión (Tabla 75).

Tabla 75: Modelo de regresión logística - Depresión y Miedo a caerse.

\begin{tabular}{cccccccc}
\hline & Estimación & $\begin{array}{c}\text { Error } \\
\text { estándar }\end{array}$ & Z-valor & p-valor & O.R. & \multicolumn{2}{c}{ IC 95\% OR } \\
(Intercept) & -2.527 & 1.1643 & -2.1703 & $\mathbf{0 . 0 3}$ &. &. & Superior \\
\hline FES-I & 0.028 & 0.0537 & 0.5211 & 0.6023 & 1.0284 & 0.9257 & 1.1424 \\
\hline $\begin{array}{c}\text { Grupo } \\
\text { Caso }\end{array}$ & 0.9966 & 0.6085 & 1.6378 & 0.1015 & 2.709 & 0.822 & 8.9276 \\
\hline
\end{tabular}

El miedo a caerse no tuvo un efecto estadísticamente significativo sobre la probabilidad de tener sintomatología depresiva considerando el grupo como factor de confusión.

\subsubsection{Relación entre la Depresión y el Dolor}

Teniendo en cuenta que la mayoría de las personas no presentó quejas de dolor, dicha variable se categorizó en: 0 : no dolor y $>0$ : con dolor. El porcentaje de los participantes que refirieron dolor en cada una de las partes del cuerpo y el resultado de la hipótesis de independencia pueden ser observados en la Tabla 76.

Tabla 76: Datos descriptivos y el contraste de independencia para la relación entre la Sintomatología depresiva y el Dolor.

\begin{tabular}{cccccccc} 
& \multicolumn{2}{c}{$\begin{array}{c}\text { No } \\
\text { sintomatología } \\
\text { depresiva }\end{array}$} & \multicolumn{2}{c}{$\begin{array}{c}\text { Posible } \\
\text { sintomatología } \\
\text { depresiva }\end{array}$} & \multicolumn{2}{c}{$\begin{array}{c}\text { Ho: } \\
\text { independencia }\end{array}$} \\
\hline Cervical & $\mathrm{N}$ & $\%$ & $\mathrm{~N}$ & $\%$ & Chi & p- valor \\
\hline Tórax & 4 & 12.31 & 5 & 33.33 & ${ }^{*}$ & 0.0610 \\
\hline Lumbar & 8 & 12.31 & 3 & 20 & ${ }^{*}$ & 0.4234 \\
\hline Cadera & 6 & 9.23 & 2 & 13.33 & ${ }^{*}$ & 0.6397 \\
\hline Rodilla & 12 & 18.46 & 1 & 6.67 & ${ }^{*}$ & 0.4433 \\
\hline Pierna & 4 & 6.15 & 1 & 6.67 & ${ }^{*}$ & 1.0000 \\
\hline
\end{tabular}

*Test exacto de Fisher 
Se utilizó el modelo de regresión logística para evaluar cómo influyó el dolor en la posibilidad de tener sintomatología depresiva (Tabla 77).

Tabla 77: Modelo de regresión logística - Depresión y Dolor.

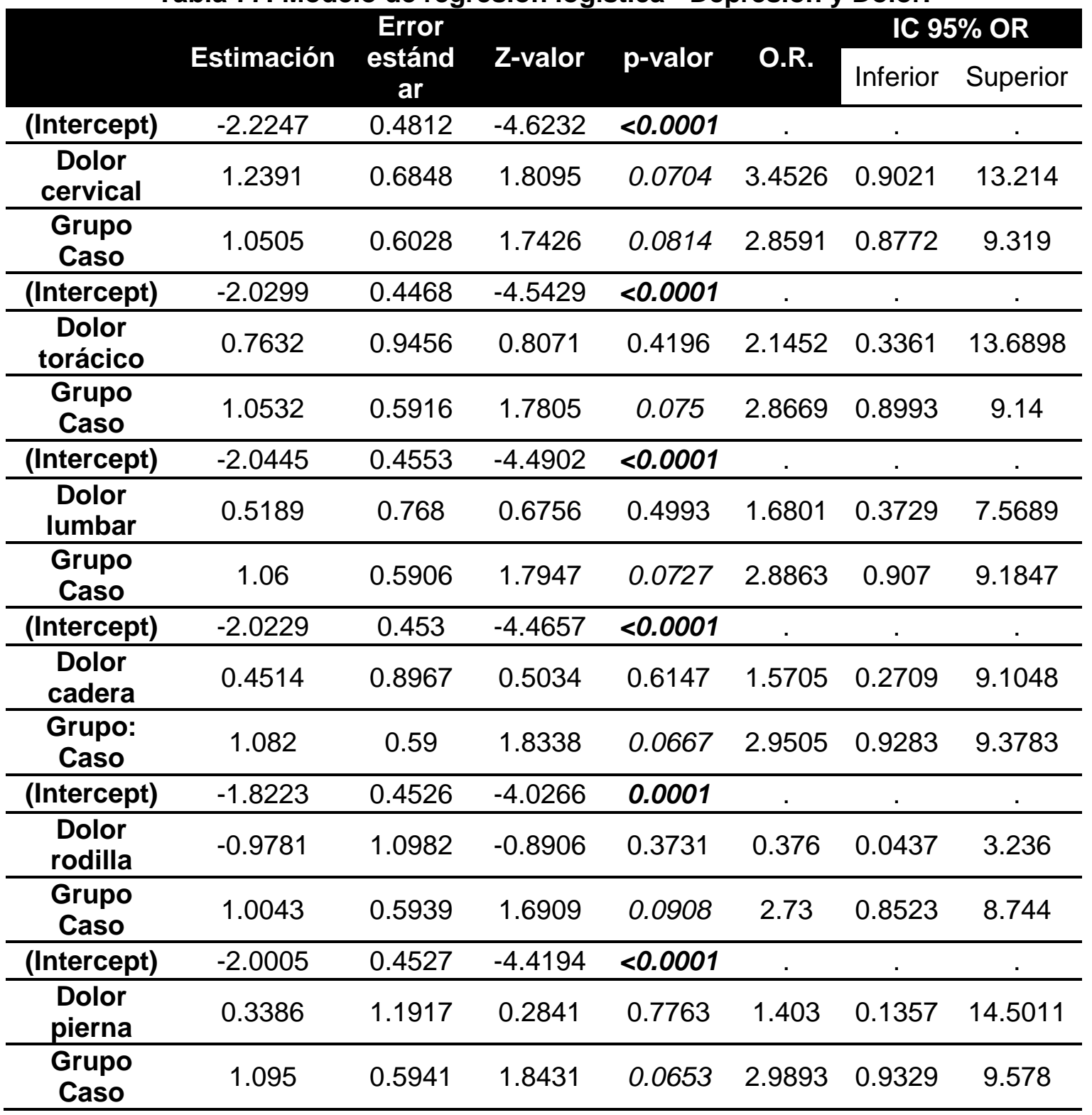

El dolor no tuvo un efecto estadísticamente significativo sobre la probabilidad de tener sintomatología depresiva cuando se consideró el grupo como factor de confusión en ninguna de las partes del cuerpo evaluadas. Las demás regiones del cuerpo fueron descartadas porque ninguno o un número muy bajo de participantes refirieron dolor en estas partes. 


\subsubsection{Análisis multivariante}

Para evaluar el efecto conjunto de algunas variables sobre la VL se construyó un modelo de regresión lineal multivariante. En él se consideraron las variables que resultaron estadísticamente significativas; FCu, ACC, AT, AL, IP y TUG además de las variables de confusión ( $S C$ y AV). Tal modelo se hizo solamente en el grupo control porque en el grupo caso no hubo relación estadísticamente significativa entre la VL y las variables evaluadas. La correlación entre las variables mencionadas puede verse en la Tabla 78.

Tabla 78: Correlación entre las variables de interés.

\begin{tabular}{cccccccc}
\hline \multirow{2}{*}{ FCu } & & FCu & ACC & AT & AL & IP & TUG \\
& rho & 1 & 0.4929 & 0.5945 & -0.0556 & 0.0333 & -0.1273 \\
\cline { 2 - 8 } & p-valor &. & $\mathbf{0 . 0 0 1 2}$ & $\mathbf{0 . 0 0 0 1}$ & 0.7331 & 0.8383 & 0.4338 \\
\hline \multirow{2}{*}{ ACC } & rho & 0.4929 & 1 & 0.3394 & -0.022 & -0.1467 & -0.0345 \\
\cline { 2 - 8 } & p-valor & $\mathbf{0 . 0 0 1 2}$ &. & $\mathbf{0 . 0 3 2 1}$ & 0.893 & 0.3662 & 0.8325 \\
\hline \multirow{2}{*}{ AT } & rho & 0.5945 & 0.3394 & 1 & 0.016 & -0.1978 & 0.0069 \\
\cline { 2 - 8 } & p-valor & $\mathbf{0 . 0 0 0 1}$ & $\mathbf{0 . 0 3 2 1}$ &. & 0.9217 & 0.2211 & 0.9664 \\
\hline \multirow{2}{*}{ AL } & rho & -0.0556 & -0.022 & 0.016 & 1 & -0.8084 & -0.2737 \\
\cline { 2 - 8 } & p-valor & 0.7331 & 0.893 & 0.9217 &. & $<0.0001$ & 0.0875 \\
\hline \multirow{2}{*}{ IP } & rho & 0.0333 & -0.1467 & -0.1978 & -0.8084 & 1 & 0.1797 \\
\cline { 2 - 8 } & p-valor & 0.8383 & 0.3662 & 0.2211 & $<0.0001$ &. & 0.2671 \\
\hline \multirow{2}{*}{ TUG } & rho & -0.1273 & -0.0345 & 0.0069 & -0.2737 & 0.1797 & 1 \\
\cline { 2 - 8 } & p-valor & 0.4338 & 0.8325 & 0.9664 & 0.0875 & 0.2671 &. \\
\hline
\end{tabular}

En ellas se realizó una selección tras incluirse las variables de confusión SC y AV. Las variables seleccionadas que resultaron estadísticamente significativas fueron el AT, el AL y el TUG y el modelo de regresión fue el siguiente:

Tabla 79: Modelo de regresión lineal - VL y AT, AL y TUG.

\begin{tabular}{cccccccc} 
& $\widehat{\boldsymbol{b}}_{\boldsymbol{i}}$ & $\mathbf{E E}$ & \multicolumn{2}{c}{ IC 95\% para $\widehat{\boldsymbol{b}}_{\boldsymbol{i}}$} & \multicolumn{2}{c}{$\mathbf{H}_{\mathbf{0}}: \widehat{\boldsymbol{b}}_{\boldsymbol{i}}=\mathbf{0}$} & \multirow{2}{*}{ VIF } \\
& & & Inf. & Sup. & t-valor & p-valor & \\
\hline (Intercept) & 844.152 & 137.444 & 563.8334 & 1124.4703 & 6.142 & $<\mathbf{0 . 0 0 0 1}$ &. \\
\hline SC & 2.69 & 3.136 & -3.7071 & 9.0867 & 0.858 & 0.3977 & 1.2425 \\
\hline AV & 24.522 & 14.844 & -5.7516 & 54.7962 & 1.652 & 0.1086 & 1.159 \\
\hline AT & -1.756 & 0.507 & -2.7905 & -0.7218 & -3.463 & $\mathbf{0 . 0 0 1 6}$ & 1.1733 \\
\hline AL & -2.833 & 0.65 & -4.1587 & -1.5077 & -4.359 & $\mathbf{0 . 0 0 0 1}$ & 1.1114 \\
\hline TUG & -4.456 & 1.624 & -7.7682 & -1.143 & -2.743 & $\mathbf{0 . 0 1}$ & 1.0608 \\
\hline
\end{tabular}

Con lo cual, por cada grado que aumentó el AT de un individuo control con la misma SC y AV y el mismo AL y TUG, la VL disminuyó de media 1.76 unidades. Por cada grado que aumentó el $A L$ de un individuo control con la 
misma SC y AV y el mismo AT y TUG, la VL disminuyó de media 2.5 unidades. $Y$, eliminando 3 sujetos por ser posibles puntos de influencia, se encontró que por cada unidad que aumentó la variable TUG de un individuo control con la misma SC y AV y el mismo AT y AL, la VL disminuyó de media 4.45 unidades.

Tabla 80: Bondad de ajuste.

$R^{2} \quad$ p-valor

$0.6<0.0001$

El modelo de regresión fue significativo estadísticamente y explicó el $60 \%$ de la variabilidad. Según la tabla ANOVA, los AT, AL y el equilibrio tuvieron un efecto estadísticamente significativo sobre la $V L(p=0.0001, p=0.0004$ y $p=0.01$, respectivamente). 
6. DISCUSIÓN 

El propósito de esta investigación fue analizar los efectos de la postura corporal, equilibrio y destreza manual sobre la $V L$ de personas mayores con y sin BV. Como objetivo secundario se comparó la postura corporal, el equilibrio, la destreza manual, la función visual, la sintomatología depresiva, el miedo a caerse y el dolor entre los dos grupos participantes. Además, se analizó las posibles relaciones entre sus niveles de sintomatología depresiva y sus dificultades posturales, motrices, VL, miedo a caerse y el dolor. En general, los resultados sugieren que la postura corporal adoptada en el momento de la lectura puede interferir en la eficacia de la misma; aunque es necesario investigar los distintos grupos de edad.

\subsection{Discusión de la metodología}

\subsubsection{Participantes}

Al empezar con la investigación nos encontramos con la primera dificultad metodológica: el tamaño de la muestra. Al igual que en otros estudios de la misma naturaleza, conseguir participantes que se ajustaran a los criterios de inclusión y que estuvieran dispuestos a participar de una investigación con una evaluación exhaustiva, no ha sido tarea fácil. Para este estudio se invitó a 174 personas, pero al final solamente 80 aceptaron participar. Seguir reclutando pacientes hubiera supuesto alargar excesivamente la investigación en el tiempo.

Las razones para negarse a participar en este estudio han sido muchas. Algunas personas tenían problemas con el transporte, otras no estaban interesadas en participar sin dar más explicaciones, no tenían tiempo para ello y otras se veían demasiado mayores para participar.

\subsubsection{Instrumentos de evaluación}

La agudeza visual de lejos fue evaluada con la tabla ETDRS y transformada en logMAR. Ya la de cerca con el test de cerca Lighthouse, Carlstadt, New York, USA. Tal test ha sido aplicado solamente a los participantes con BV, con el fin de averiguar la necesidad del uso de ayudas ópticas. Para medir el campo visual se utilizó la campimetría de Humphrey en grados centrales residuales cuándo se trata de un defecto de restricción concéntrica del CV. Y para la sensibilidad al contraste, el test de Pelli-Robson, por el número de ternas vistas con el ojo derecho, con el izquierdo y con ambos.

La elección de las pruebas se basa en su utilización previa en otros estudios $^{202-205}$.

Una vez finalizadas dichas pruebas se realizó el Examen Cognoscitivo Minimental de Folstein et al. ${ }^{191}$ y adaptada al español por Lobo et al. ${ }^{185}$. Su aplicación tenía el objetivo de comprobar si los participantes cumplían el criterio 
de exclusión, es decir, si padecían deterioro cognitivo. Es una herramienta ampliamente utilizada en clínica e investigación ${ }^{206-208}$, es breve y sencilla, además el proceso de corrección puede ser ajustado a las personas mayores con discapacidad visual, razones por las cuales ha sido elegida para este estudio ${ }^{192}$.

En cuanto a la evaluación de la sintomatología depresiva de los participantes, se seleccionó la escala de Yesavage, a pesar de la amplia gama de tests utilizados con frecuencia como es el caso de la Escala de Depresión del Centro de Estudios Epidemiológicos (CES-D) ${ }^{209}$, la Escala Autoaplicada para la Depresión de Zung (SDS) ${ }^{210}$, el Inventario de Depresión de Beck ${ }^{211}$. Sin embargo, están más orientados a colectivos con otro perfil y no a personas con edad avanzada como las de esta muestra. Además de eso la GDS es la empleada en la mayoría de los estudios de personas mayores con discapacidad visual ${ }^{212,213}$. La versión abreviada de esta escala utilizada para este estudio, fue validada por Martínez et al. en el 2002 la cual presentó parámetros de fiabilidad y validez aceptables y similares a los del cuestionario original. Su investigación contó con la participación de 249 personas mayores, con media de edad de 74.3 años y los resultados apuntan una fiabilidad intraobservador de 0.95 , interobservador de 0.65 y con una consistencia interna de 0.99 .

El siguiente test aplicado fue la FES-I. En la actualidad hay muchos tests que miden el miedo a caerse como: The Survey of Activities and Fear of Falling in the Elderly (SAFE) ${ }^{214}$, Activities-specific Balance Confidence scale ${ }^{215}$, Concern about Falling Scale ${ }^{216}$ entre otros. Sin embargo, la razón por utilizar la FES-I es por su comprobada validez y fiabilidad para evaluar el miedo de caer de las personas mayores, además su versión en inglés ha sido traducida a 14 idiomas pudiendo ser utilizada para fines de investigación y clínicos en diferentes muestras de diversos países ${ }^{217-219}$.

La última escala administrada a los participantes fue la EVA. El dolor debe medirse con un instrumento válido y fiable que aporte resultados consistentes en todos los niveles del dolor, lo que es el caso de la EVA ${ }^{220}$. Existen muchas variaciones de esta escala y todas se consideran equivalentes a la original ${ }^{197}$, sin embargo en este estudio se optó por la original por su fácil adaptación a la hora de evaluar a las personas con BV.

Al finalizar los tests descritos anteriormente se inició las evaluaciones físicas, empezando por el TUG. Ha sido extensamente utilizado en investigaciones sobre velocidad de la marcha, equilibrio e habilidad funcional en la realización de tareas diarias en personas mayores ${ }^{221-223}$. Es considerada una herramienta válida y fiable e igualmente rápida y de fácil ejecución ${ }^{183}$.

En cuanto a la evaluación de la destreza manual, llevada a cabo con el Pick Up Test, no existe un test estándar para su evaluación, con lo cual hay una variedad de pruebas disponibles y su elección depende de lo que se aspira 
evaluar. No está disponible a la venta, pero es fácilmente reproducible y barato de adquirir. Su elección para este estudio fue por ser una herramienta fiable, sensible a los cambios clínicos, sencilla, de fácil manejo y ejecución y por poseer valores de referencia en categorías distintas de edad basados en el género, la mano dominante, y los ojos abiertos y cerrados ${ }^{153,224,225}$.

Los instrumentos elegidos para la evaluación postural fueron la fotogrametría y el acelerómetro. La fiabilidad y validez de ambos fueron comprobadas por numerosos estudios en los más diversos segmentos corporales ${ }^{107,109,112,226-228 . ~}$ La opción por el software de evaluación postural (SAPO) para este estudio fue debido a su fácil manejo, ya que se acompaña de tutoriales científicos y está disponible al dominio público (http://sapo.incubadora.fapesp.br). Y el LUMOback por ser un aparato novedoso, de fácil manejo y rápida ejecución, lo que facilita el procedimiento de evaluación postural en la práctica clínica.

La última prueba realizada fue el IReST. En la literatura se ha encontrado otros tests de lectura como el MNread Reading Test y el Radner Reading Charts ${ }^{229}$, sin embargo se optó por el IReST por sus diferentes textos de fácil comprensión y está traducido a 17 idiomas, con lo cual permite una evaluación estandarizada de la VL y facilita los estudios entre países ${ }^{182}$. Además, tal herramienta ha sido utilizada en muchos otros estudios ${ }^{140,230,231}$.

\subsection{Discusión de los resultados}

\subsubsection{La muestra}

La desigualdad en la cantidad de participantes en cada grupo fue evidente (caso $=39 \%$ y control $=61 \%$ ) y el menor número en el grupo caso puede ser justificado por diferentes razones: la imposibilidad para desplazarse autónomamente, la dificultad para que sus familiares pudieran acompañarle y el hecho de vivir fuera de la provincia.

De los 80 pacientes que aceptaron participar en el estudio el $61.25 \%$ fueron mujeres. Este dato puede deberse a que las mujeres tienden a ser más participativas y tienen una mayor motivación intrínseca para realizar actividades que les permitan relacionarse y acceder a la información y por eso es posible que acudan con más frecuencia a los centros de día y a los servicios de rehabilitación visual ${ }^{232}$. Además, la predominancia de participantes del sexo femenino en ambos grupos se justifica por el hecho de que las mujeres ocupan una gran parcela de la población, tienen mayor longevidad y muchas enfermedades oculares son intrínsecamente más prevalentes en las mujeres $^{233}$.

Otro punto importante es la edad. A pesar de que hubo variabilidad entre las edades de la muestra principalmente en el grupo caso, al comparar las medias de las edades de los grupos caso (72.84 años) y control (71.88 años) no se 
encontraron diferencias estadísticamente significativas, con lo cual no fue un factor importante en la comparación entre los grupos.

La obesidad es una variable que está directamente relacionada con la edad, y según la Encuesta Europea de Salud en España realizada por el INE ${ }^{234}$ la mayoría de las personas con más de 65 años tienen sobrepeso (>40\%). Estos datos explican el porcentaje de participantes de este estudio con sobrepeso $(41,25 \%)$, además refuerzan su relación con la edad teniendo en cuenta que los participantes incluidos en este estudio pueden considerarse bastantes mayores ya que tienen una media de edad de 72.25 \pm 6.03 .

En lo que se refiere al miembro dominante, casi la totalidad de los participantes eran diestros (98.75\%). Hay estudios que señalan que la mayor parte de la población es diestra y que la predilección por una de las manos es heredable, pero puede ser influenciada por diversos factores como el medio ambiente, genética o cultura entre otros. Esto podría explicar las quejas de algunos participantes sobre la prohibición de utilizar la mano izquierda en la infancia, por parte de los profesores en el colegio y de los padres en casa ${ }^{235-237}$.

No hubo diferencias estadísticamente significativas entre los grupos en lo que se refiere a las variables miembro dominante, estado civil e IMC. Es decir, los dos grupos tenían características semejantes para las variables mencionadas, con lo cual el hecho de tener o no BV podría no interferir en las mismas.

Como cabría esperar, la AV en logMAR fue significativamente mayor en el grupo de casos ya que el mismo está compuesto de personas con BV. Así mismo con la SC, el valor del Pelli-Robson fue significativamente menor en el grupo caso. Tanto los valores de la media cuanto del rango encontrados se asemejan mucho a los hallados en el estudio de Haymes y Chen ${ }^{238}$.

\subsubsection{Fiabilidad y validez del sensor postural}

En la actualidad, existen diferentes aplicaciones médicas para los teléfonos inteligentes y el acelerómetro del iPhone es el más investigado. En un estudio que investigó la fiabilidad de una aplicación basada en teléfonos inteligentes para medir la gibosidad en la escoliosis, se concluyó que el iPhone es fiable $(\mathrm{CCl}=0.92)$ para evaluar esta variable en sujetos con escoliosis ${ }^{239}$. Por otra parte, el sesgo entre el iPhone y las mediciones del Scoliometer fue 2.1ㅇ (1.6\%). Otro estudio comprobó la validez y fiabilidad de un sensor de movimiento del tronco (Spineangel $\Theta^{\circledR}$ ) encontrando una alta fiabilidad ( $\mathrm{CCl}: 0.90$ a 0.97 y CV: 3.4 a $5.2 \%)^{240}$. El sesgo entre este sensor y un sistema de análisis de movimiento osciló entre $1.7^{\circ}\left(3.2^{\circ}\right)$ y $22.8^{\circ}\left(7.8^{\circ}\right)$. El estudio realizado por Balg et al. ${ }^{241}$ evaluó la fiabilidad y la validez de la aplicación para iPhone Scoligauge. Se hicieron dos medidas para evaluar la fiabilidad intraobservador que resultó ser alta $(\mathrm{CCl}=0.96, \mathrm{p}<0.001)$. Además, presentó un sesgo entre dicha aplicación y el escoliómetro de $0.4^{\circ}\left(3.1^{\circ}\right)$. 
La fiabilidad del acelerómetro también fue evaluada en un estudio para medir del rango de movimiento (ROM) de la rodilla, encontrando un excelente coeficiente de correlación intra-clase $(\mathrm{CCl}=0.98)$ para las mediciones con el APP ${ }^{242}$. En nuestro estudio, los valores de fiabilidad y variabilidad observados fueron muy similares a los reportados en los estudios citados. Sin embargo, los sesgos detectados aquí fueron mucho más bajos que los documentados en los artículos citados. De este modo, se podría afirmar que la LUMOback es una herramienta fiable.

En lo que respecta al coeficiente de variación, los resultados indican un poco de disparidad entre las mediciones realizadas en el mismo día de menos de $3 \%$. Un bajo CV refleja la homogeneidad entre los valores medidos, lo que demuestra la eficacia y la precisión del dispositivo de medición. Debido a que el coeficiente de variación de $5.5 \%$ es considerado como una moderada a buena concordancia de las medidas para el rango de movimiento de la columna lumbar, se puede concluir que nuestros resultados de variabilidad son muy consistentes ${ }^{243}$.

En lo que respecta a la validez concurrente, hubo una relación fuerte y positiva entre las mediciones del LUMOback y las tomadas con la fotogrametría demostrando una alta validez entre los dos métodos (Gráfico 4). En nuestro conocimiento actual, la validez de LUMOback en la evaluación postural no ha sido comprobada. Sin embargo, un estudio realizado por Kooiman et al. examinaron la fiabilidad y validez del LUMOback para medir el número de pasos, tanto en condiciones de laboratorio como de vida libre. La validez se evaluó mediante la comparación del LUMOback con el sistema de OptoGait de laboratorio y ActivPAL para las condiciones de vida libre. La fiabilidad testretest del LUMOback fue excelente $(\mathrm{CCl}>0.90)$ y su error porcentual fue de $0.4 \%$ comparado al método estándar. Este estudio demostró que el LUMOback es una herramienta fiable y valida en la cuantificación del número de pasos en ambas condiciones ${ }^{244}$.

Varios datos sobre la validez de diferentes sistemas para la evaluación postural fueron mencionados en algunos estudios. Por ejemplo, un estudio sobre la comparación entre el BodyGuard (un monitor inalámbrico) y un sistema basado en el laboratorio $(C O D A)$ demostró alta relación $\left(R^{2}=0.78\right)$ para una variedad de tareas sentado y de pie $^{245}$. En otro estudio, se utilizó el acelerómetro para estimar los ángulos de inclinación de la pelvis y se encontró una alta correlación $\left(R^{2}=0.86\right.$ flexión y extensión $\left.R^{2}=0.67\right)$ con la inclinación pélvica obtenida a partir de un sistema de análisis de movimiento ${ }^{240}$. Además, Balg et al. encontraron en su estudio sobre la validez y fiabilidad del iPhone para medir la escoliosis, un sesgo de $0.4^{\circ}$ con LA de $95 \%$ de $3.1^{\circ}$ en el gráfico de BlandAltman $^{241}$. Y al evaluar la fiabilidad y validez del Microsoft Kinect para medir la postura de la cabeza, Oh et al. hallaron en el Bland-Altman un sesgo de $0.04^{\circ}$ a $1.75^{\circ}\left(0.51^{\circ}\right.$ a $\left.1.20^{\circ}\right)$ con un LA $95 \%$ de $-2.36^{\circ}$ a $+2.47^{\circ}$ para la inclinación lateral de la cabeza ${ }^{246}$. La comparación de nuestros resultados con los datos 
anteriores sobre la validez concurrente en las evaluaciones posturales, obtuvieron mayores valores del coeficiente de Pearson y las diferencias de las medias fueron menores, por lo tanto, las herramientas utilizadas en este estudio son válidas y equivalentes.

\subsubsection{Fiabilidad en la demarcación de los puntos anatómicos}

El método de evaluación de la postura corporal es algo que viene sufriendo cambios a lo largo del tiempo. En la actualidad, los métodos cuantitativos son los más elegidos para la investigación por su perspectiva menos subjetiva y personal. Para obtener un diagnóstico más preciso, es esencial que el método elegido sea fiable y válido. El método de evaluación postural utilizado en este estudio ha sido la fotogrametría, que tiene su fiabilidad y validez comprobada por diversos estudios ${ }^{100,111,247,248}$.

Para la evaluación de la postura en este estudio se utilizó la técnica de palpación para encontrar los puntos anatómicos, la cual ha sido realizada también en otros estudios ${ }^{72,247-249}$. Sin embargo, este método aumenta la posibilidad de error cuando es aplicado por más de un examinador, pues cada uno puede tener una interpretación distinta de la localización de un punto anatómico 250 . Por lo que el tipo de marcadores utilizados para demarcar los puntos anatómicos y la cantidad de evaluadores pueden comprometer la fiabilidad de los resultados. Hay estudios que comprueban la diferencia en la fiabilidad del procedimiento de demarcación de los puntos anatómicos, cuando realizado por más de un examinador 249,251 . Con lo cual, para esta investigación se hizo la evaluación de los ocho ángulos simultáneamente por el mismo evaluador, minimizando las posibilidades de error.

Con el fin de examinar la fiabilidad de nuestro método, se evaluó la concordancia entre días. Los resultados del análisis de la reproducibilidad de las medidas posturales comprobaron la normalidad tanto de la medida 1 como la medida 2, como también que las diferencias entre las mediciones proceden de una distribución normal. Para el contraste de normalidad se utilizó el test de Shapiro-Wilk, muy utilizado sobre todo en muestras pequeñas $(n<50)$. Además, la media y mediana fueron casi iguales en los ocho ángulos medidos, así que la diferencia entre ellas fue próxima a cero. Teniendo en cuenta que la media de las desviaciones típicas intra-sujeto (DT de las dos medidas en cada sujeto) y el coeficiente de variación presentaron estimaciones pequeñas $(C V<1 \%)$, el margen de error entre las mediciones se considera aceptable. EI CV para la FT fue el único por encima de $1 \%(\mathrm{CV}=6.57 \%)$, sin embargo una variabilidad menor del $10 \%$ es considerada como admisible por la literatura científica ${ }^{252}$.

Por último, se evaluó la proporción de la variabilidad total del acuerdo entre días, valor indicado por el $\mathrm{CCl}$. Para todos los ángulos medidos el $\mathrm{CCl}$ fue $+98 \%$, por lo que el acuerdo entre días para dichas medidas fue muy bueno ${ }^{253}$. 
Teniendo en cuenta estos valores, la variabilidad observada en la muestra se podría explicar a las diferencias entre sujetos y no por las diferencias entre las mediciones.

6.2.4. Discusión de los resultados de la Postura en sedestación: comparación entre grupos

Cuando se habla de análisis comparativa de postura, es difícil encontrar valores de referencia fiables que puedan ser utilizados como parámetros para determinar la normalidad y anormalidad postural ${ }^{187}$.

Algunos estudios describen valores medios de los ángulos encontrados para ciertos segmentos del cuerpo, sin embargo las metodologías de las evaluaciones de dichos trabajos son distintas, no presentando un patrón único de evaluación ${ }^{21,59,76,254-256}$. La ausencia de una norma de clasificación tiene despertado el interés de muchos investigadores, los cuales vienen adoptando métodos cuantitativos, medidos en grados.

De hecho, es difícil definir parámetros de referencia teniendo en cuenta las variaciones anatómicas, las alteraciones adquiridas por la edad y el propio sistema fisiológico del envejecimiento. Por esto en esta investigación se utilizó como parámetro comparativo las variables del grupo control, que poseen características similares disminuyendo las posibilidades de error.

Las diferencias aparentes en las medidas posturales entre los dos grupos (caso-control) son más evidentes en el grupo caso para la FC $\left(88^{\circ}\right.$ $\left.74.14^{\circ}=13.86^{\circ}\right)$, Fcu $\left(75.95^{\circ}-65.20^{\circ}=10.75^{\circ}\right)$, FT $\left(34.95^{\circ}-24.11^{\circ}=10.84^{\circ}\right)$, AT $\left(237.95^{\circ}-243.46^{\circ}=-5.51^{\circ}\right)$ e IP $\left(29.60^{\circ}-22.86^{\circ}=6.74^{\circ}\right)$. Esto sugiere que los sujetos con BV tenían una tendencia a mantener su columna en más flexión cuando se centraba en la lectura del texto.

El ángulo de la FC está relacionado con el ángulo de la mirada y el de la FCu con la posición de la cabeza en relación con la cervical. El grupo caso en este estudio manifestó una posición de la cabeza más hacia adelante con una mayor flexión cervical inferior y la mirada más hacia abajo que el grupo control. Dichas alteraciones posturales encontradas en el grupo caso pueden estar asociadas a los cambios adaptativos que dicha población tiene que hacer para lograr leer, como la reducción de la distancia de trabajo, la utilización de las ayudas ópticas y las posturas adoptadas para optimizar la lectura ${ }^{22,84}$.

Tales resultados concuerdan con los encontrados por Szeto et al. ${ }^{23}$ tras evaluar la postura de la cabeza y del cuello en trabajadoras de la informática. Todos los participantes presentaron un aumento de aproximadamente $10 \%$ en la postura de la cabeza hacia adelante desde la postura sentada relajada a la de trabajo con la pantalla del ordenador. 
El ángulo FT también fue mayor en el grupo caso y lo que podría explicar tal hallazgo es la estrecha relación que existe entre el mismo y la región cervical. Hay estudios que afirman que la cifosis torácica está relacionada con alteraciones del cuello y que incluso dicha relación es llevada en cuenta en la rehabilitación de disfunciones cervicales ${ }^{256,257}$.

En lo que se refiere al AT, O'Sullivan et al ${ }^{119}$. evaluaron la postura sentada en 1596 adolescentes utilizando los mismos puntos anatómicos utilizados en este estudio para dicho ángulo. El grado de inclinación encontrado fue menor $\left(232^{\circ}\right)$ que el encontrado en nuestro estudio en ambos grupos, lo que posiblemente es resultado de la diferencia de edad entre las poblaciones estudiadas. Se encontró en nuestro estudio que el AT en el grupo control fue mayor que en el grupo caso, lo que es un poco contradictorio según el estudio mencionado anteriormente, cuanto más encorvada es la postura mayor debería ser el AT. Sin embargo, en consecuencia del acercamiento del cuerpo hacia la mesa de trabajo y el objeto de lectura, las personas con BV realizaron una mayor inclinación del tronco hacia delante pero no encorvamiento, produciendo una rectificación de la región lumbar y disminución de la $\mathrm{IP}^{258}$.

\subsubsection{Discusión de los resultados de la Postura en bipedestación: comparación entre grupos}

Al comparar las posturas adoptadas por los participantes de ambos grupos en bipedestación, se observó que los del grupo caso tenían la cabeza más hacia arriba que los participantes del grupo control. Tal hallazgo se explica por el hecho de que el deterioro o ausencia de la visión conduce a alteraciones posturales de la cabeza. Conforme demostraron Sanchez et al. ${ }^{68}$ en su estudio, al encontrar que las personas con DV congénita presentan diferencias en la protrusión de la cabeza cuando comparadas a las personas sin DV. Lo que corrobora el estudio hecho por Fjellvang y Solow ${ }^{69}$, en el cual afirman que la postura de la cabeza de las personas con DV se diferencia de la adoptada por las personas con agudeza visual normal. Tal hecho es explicado por Zhang ${ }^{73}$ cuando afirma que la postura compensatoria de la cabeza generalmente se adopta para optimizar la AV o la obtención de la visión binocular.

En concordancia con dichas alteraciones, Aulisa et al. ${ }^{70}$ sugieren que en la ceguera congénita el desarrollo de pequeñas curvas de la columna vertebral es simplemente la respuesta de un mecanismo del cuerpo para alcanzar el equilibrio estable en la misma. Del mismo modo, Silva et al. ${ }^{72}$ también describen que la DV conduce a un aumento de la asimetría postural, resultado de una postura anormal de la cabeza con el fin de mejorar el ángulo de la visión.

Con relación al cuello, al comparar los dos grupos se observó una mayor inclinación hacia delante en los participantes del grupo caso. La mayor 
inclinación del cuello, en la mayoría de los casos puede estar asociada a las posturas incorrectas al hacer uso de ayudas ópticas, como las lupas, gafas de cerca u otras. Según Krueger et al. ${ }^{16}$, las personas que utilizan lupas refieren a menudo más quejas musculoesqueléticas que los que no las utilizan.

Para el ángulo cráneo-cervical, los participantes del grupo caso presentaron una postura cervical más hacia delante y hacia arriba que los controles. Esto se razona por el hecho de que los ángulos del cuello y de la cabeza están relacionados. Es decir, si el cuello está posicionado hacia delante y la cabeza hacia arriba, el resultado del ángulo cráneo-cervical es la junción de los dos ángulos. En la posición estática la postura de cada segmento de la columna está relacionada, puesto que la alteración de la postura en una determinada región de la columna puede alterar la postura en otro segmento. Por ejemplo, una postura de la cabeza hacia delante viene acompañada por una flexión de la región cervical inferior y una extensión de la región cervical superior ${ }^{52}$.

La flexión torácica fue el único ángulo medido que se quedó al borde de la significación, siendo mayor en las personas con BV. A pesar de que, hasta la actualidad, no se conocen estudios que expliquen tal hallazgo para esta población específica, hay algunos que relacionan la tendencia al aumento de la cifosis torácica en bipedestación con el predominio de posturas en flexión del tronco en sedestación. Es decir, tras evaluar los posibles cambios posturales en 15 esquiadores de elite por 5 años, Alricsson y Werner ${ }^{259}$ han encontrado cambios posturales en las columnas torácica y lumbar relacionados con surgimiento de dolor de espalda en 8 de ellos. Los demás no presentaron cambios posturales significativos ni dolor y realizaban otras actividades deportivas además del esquí. En cuanto que Rodríguez et al. ${ }^{260}$ encontraron un elevado porcentaje de casos con hipercifosis torácica al evaluar la postura en bipedestación de ciclistas de las categorías élite y máster 30 .

Las medidas posturales del ángulo cérvico-torácico no han sido muy investigadas ya que la mayoría de los estudios en esta área se han restringido a la columna cervical. La terminología utilizada para representar esta postura no ha sido estandarizada, así que los puntos anatómicos utilizados como referencia para dicho ángulo difieren según el estudio. En este estudio no se encontraron diferencias estadísticamente significativas entre ambos grupos para el ACT, de hecho la diferencia de medias caso-control fue de $0.67^{\circ}$. Teniendo en cuenta que la postura de la columna torácica es afectada por las posiciones de la región inferior de la espalda y de la pelvis ${ }^{52}$, la igualdad de medias en las medidas del ángulo torácico y de la inclinación pélvica (casocontrol $=0.01^{\circ}$ y caso-control $=0.86^{\circ}$, respectivamente) pueden haber influenciado la medida encontrada para el ACT.

El AL menor (grupo control) es resultante de una basculación anterior de la pelvis, lo que da lugar una mayor lordosis lumbar. En contrapartida, la basculación posterior de la pelvis resulta en un mayor AL (grupo caso). A pesar 
de que la diferencia de las medias del $A L$ en ambos grupos ha sido un poco mayor (caso-control $=1.76^{\circ}$ ), tampoco fue estadísticamente significativa.

A pesar de que, desde el punto de vista estadístico, no hubo diferencias significativas entre los grupos, desde el punto de vista clínico se puede decir que los resultados de este estudio apuntan a una alteración de la postura corporal entre el grupo caso y el control que puede desarrollar disfunciones musculoesqueléticas. Además, el hecho de que las diferencias entre los grupos no tuvieron significancia estadística, podría ser debido al pequeño tamaño de la muestra.

6.2.6. Discusión de las variables físicas equilibrio, destreza manual y velocidad lectora: comparación entre ambos grupos

Muchos estudios han evaluado el efecto de la DV en el equilibrio, principalmente en el estático, y sus resultados afirman que la primera está asociada con una reducción del control postural ${ }^{128,129,261,262}$. Sin embargo, son pocos los que evalúan el equilibrio dinámico comparando grupos de personas con y sin DV263-265. Hay muchos tests disponibles para evaluar el equilibrio y, a pesar de que el TUG es una herramienta ampliamente utilizada para evaluar la movilidad funcional, así como para predecir las caídas en personas mayores, hay muchos resultados contradictorios en la literatura.

Empezando por un estudio con 102 adultos mayores, los cambios sutiles en la velocidad de la marcha evaluados sobre una distancia de 6 metros fueron más eficaces en la predicción de caídas que un mal desempeño en el TUG ${ }^{266}$. Un factor importante que contribuye para el surgimiento de resultados contradictorios es las metodologías utilizadas en los diferentes estudios. Los diferentes diseños de investigación al evaluar el desempeño en dicha prueba pueden ser importantes, además de otros factores como el tipo de población estudiada y la falta de control de los factores de confusión potenciales. Los diferentes estudios reflejan variaciones en la administración y las condiciones de control del TUG, es decir, las diferencias en cuanto a la condición de ritmo y tipos de sillas utilizadas. Algunos estudios utilizaron un ritmo rápido para el test, mientras que otros utilizaron el ritmo de costumbre o no proporcionaron ninguna información.

La puntuación obtenida en el TUG está relacionada al tiempo de realización de la misma en segundos. Las personas que la realizan en menos de 20 s son consideradas independientes para tareas básicas ${ }^{183}$. En un estudio con mujeres de edad avanzada, Bischoff et al. encontraron que el $92 \%$ de las mujeres estudiadas realizaron la prueba TUG en menos de 12 segundos ${ }^{267}$. Sin embargo, al estudiar una población de personas mayores con DV, Kovács et al. encontraron un valor medio de 20 s en la realización del TUG, mejorando a $17 \mathrm{~s}$ después de un programa de ejercicios ${ }^{268}$. Cheung et al. también refirieron altos 
valores para el TUG (27s) en una población mayor con DV y un cambio significativo (23s) después de un programa de ejercicios específicos ${ }^{269}$. A pesar de la relación encontrada entre la mala visión y un peor equilibrio corporal en algunas investigaciones anteriores, este estudio no pudo confirmar estos hallazgos. Aunque al comparar los dos grupos, las personas mayores con BV han tenido valores más altos en el TUG que las personas sin BV, tales diferencias no han sido estadísticamente significativas.

El hecho de no concordar nuestros resultados con los de otros estudios puede deberse al nivel de la DV. Los estudios mencionados refieren una AV muy baja (6/120) o no especifican la AV de los participantes ${ }^{268,269}$. Con lo cual, la diversidad de los resultados podría estar relacionada con la heterogeneidad clínica de las poblaciones consideradas.

En lo que se refiere a la destreza manual, un estudio realizado en 2007 ha sido el primero a evaluar la ejecución en el Pick Up test de personas mayores sanas $^{153}$. Participaron de dicho estudio 116 personas entre 20 y 89 años de edad, las cuales fueron divididas en tres grupos: i) jóvenes (20-39 años); ii) mediana edad (40-59 años); y iii) mayor ( $\geq 60$ años). Los resultados encontrados para los tres grupos en la mano dominante/OA fueron 12.15s, $14.55 \mathrm{~s}$ y $16.4 \mathrm{~s}$ respectivamente y para la mano no-dominante/OA fueron $13.25 \mathrm{~s}, 15.65 \mathrm{~s}$ y $16.7 \mathrm{~s}$ respectivamente. Tales datos sugieren que la destreza manual disminuye con la edad y que la mano dominante es más rápida que la no-dominante al realizar el Pick Up test ${ }^{153}$.

Otro estudio que evaluó la destreza manual a través del Pick Up test fue el realizado por Amirjani et al. ${ }^{270}$ en 2011. Tras aplicar dicha prueba a 116 personas, las cuales fueron divididas en tres grupos utilizando la misma metodología del estudio citado anteriormente, se encontró mejores resultados. Es decir, con la mano dominante/OA los tres grupos tardaron $11.2 \mathrm{~s}, 12.6 \mathrm{~s}$ y $13.8 \mathrm{~s}$ respectivamente y para la mano no-dominante/OA $12.1 \mathrm{~s}, 13.1 \mathrm{~s}$ y $14 \mathrm{~s}$. Sus resultados ratifican que la destreza manual disminuye con la edad y que la mano dominante es más rápida que la no-dominante.

Así que, los resultados encontrados en este estudio concuerdan con los descritos en los estudios anteriores al encontrar una mejor destreza manual en la mano dominante y similares tiempos de ejecución en dicha prueba.

Para la realización de la prueba se requiere la capacidad de percibir el contacto con el objeto (para localizarlos) y la precisión en el agarre (para recogerlos). Con los ojos abiertos, la visión puede compensar en cierta medida alguna falla cutánea sin embargo, con los ojos cerrados, los sujetos dependen totalmente de la sensibilidad digital para localizar objetos, con lo cual requiere más tiempo para su realización ${ }^{224}$. Tal hecho podría explicar porque ambos grupos obtuvieron tiempos de ejecución similares para dicho test con los ojos cerrados. 
A pesar de que, para nuestro conocimiento, hasta el día de hoy no se conoce ningún estudio que averigüe la relación entre la BV y la destreza manual a través de la comparación entre grupos de personas mayores con y sin BV, se sabe que el deterioro visual puede influir en la destreza manual de las personas mayores ${ }^{156,271}$. En un estudio que evaluó el efecto de la ambliopía en la motricidad fina en un grupo de 82 niños con edad media de $8.2 \pm 1.7$ años, se encontró que las habilidades motoras finas en dichos niños estaban más reducidas en comparación con los sujetos control. Además, las deficiencias en el rendimiento del motor fueron mayores en las tareas de destreza manual que requieren velocidad y precisión ${ }^{271}$. Song ${ }^{156}$, en su estudio realizado en 2015 también encontró una relación entre la percepción visual y la destreza manual en personas mayores, sugiriendo que la mejora de la función visual en los adultos mayores se asocia con la mejora de la destreza manual.

Con lo cual, el hecho de que en este estudio las personas con BV tardaron más tiempo para realizar Pick Up test con los ojos abiertos que las personas sin BV se justifica.

La siguiente variable física comparada entre los grupos fue la VL. La edad es uno de los factores que influencia a la VL. Hahn et al. ${ }^{139}$ evaluaron el rendimiento de lectura de dos grupos de lectores con visión normal: 100 adultos jóvenes (18-35 años) y 100 personas mayores (60-85 años) en 4 idiomas distintos (Inglés, finlandés, francés, y alemán) utilizando el IReST. Para todos los idiomas evaluados, el grupo de lectores de más edad mostró una VL significativamente inferior que el otro grupo evaluado.

Además de la edad, la visión es otro factor de gran influencia en la VL. En su estudio, Rubin y Feely ${ }^{272}$ utilizaron el IReST para evaluar la VL de 40 personas mayores con DMAE utilizando sus ayudas ópticas adecuadas. La VL media encontrada fue de $38 \mathrm{ppm}$. En el mismo año Nguyen et al. ${ }^{13}$ realizaron un estudio con el objetivo de evaluar la capacidad de lectura antes y después de la prestación de las ayudas de baja visión adecuadas. Participaron de tal estudio 530 personas mayores ( $82 \pm 8$ años) con DMAE, en el cual se evaluó la VL a través del IReST. La VL media fue de $20 \pm 33$ ppm antes y $72 \pm 35$ ppm después de la adaptación de las ayudas visuales.

Los datos resultantes de las investigaciones citadas concuerdan con nuestros hallazgos, dónde la VL en las personas mayores con BV fue significativamente más baja que en las personas sin BV. En dichas personas los errores durante la lectura son, por supuesto, mucho más frecuentes y deben ser tomados en cuenta a la hora de medir la VL. Sin embargo, se encontró en este estudio un mejor valor en la VL (47.34 ppm) cuando comparado al estudio de Rubin y Feely ${ }^{272}$. Lo que explicaría tal diferencia, es que las patologías visuales con restricción del CV central suelen afectar más el proceso de lectura que las patologías visuales con restricción del CV periférico ${ }^{15,30}$. Y la población evaluada en dicho estudio presentó la DMAE como única patología visual, 
mientras que las personas mayores que forman parte del grupo caso en este estudio tienen patologías visuales diversas. Cuando se compararon al de Nguyen et al. ${ }^{13}$ en nuestro estudio se encontró una peor VL, no obstante el hecho de que en su estudio los pacientes fueron entrenados en cómo manejar la ayuda óptica prescrita, mientras que en nuestro estudio no explicaría tal diferencia.

6.2.7. Discusión de los resultados de las variables visuales: comparación entre grupos

Según datos epidemiológicos actuales, a nivel mundial las principales causas de DV son los errores de refracción no corregidos y las cataratas, con un $43 \%$ y un $33 \%$ respectivamente. Acompañadas del glaucoma, el $2 \%$, la degeneración macular relacionada a la edad y la retinopatía diabética ${ }^{273,274}$. Tales datos coinciden con el perfil de las patologías visuales encontradas en los participantes del grupo caso en este estudio.

Dichas patologías pueden conducir a diferentes limitaciones visuales como: disminución de la AV, defecto del CV central, constricción periférica del CV o una combinación de las anteriores ${ }^{275}$. Con lo cual, el hecho de que las personas del grupo caso presentaron una AV (logMAR) significativamente mayor que las del grupo control era esperado. Las ayudas ópticas de aumento son comúnmente prescritas para ayudar a las personas con BV a mantener su capacidad de leer. En muchos estudios que tenían como objetivo evaluar la lectura en personas mayores con BV, los participantes utilizaban las ayudas ópticas adecuadas para así ser capaces de leer ${ }^{13,143,276}$. Teniendo en cuenta la AV de los participantes del grupo caso, la mayoría de ellos utilizaron ayudas ópticas para leer, lo que concuerda con los estudios señalados.

Hay una estrecha correlación entre la AV y la sensibilidad al contraste, dónde la segunda presenta una disminución sustancial en personas con BV. Además, la SC proporciona información adicional a la $\mathrm{AV}$, muy útil en el proceso de rehabilitación ${ }^{277,278}$. Dicha correlación y la influencia de la BV en la SC explica el hecho de que la SC del grupo caso fue significativamente más baja que la del grupo control.

6.2.8. Discusión de los resultados de las variables psicológicas: comparación entre grupos

\subsubsection{Sintomatología depresiva en ambos grupos}

Las estimaciones de la prevalencia de síntomas depresivos van desde aproximadamente el $4 \%$ y el $20 \%$ de los adultos mayores de 65 años. La variabilidad en tales estimaciones resulta de si la DV se basa en datos de autoinforme o evaluación clínica ${ }^{279}$. En un estudio que investigó los síntomas 
clínicamente relevantes de la ansiedad y depresión en personas mayores con $\mathrm{BV}$, los porcentajes de los síntomas de depresión para las personas con BV fueron $14.2 \%$ mientras que en la población mayor sin BV fue de $6.2 \%$. Concluyendo que la pérdida de la visión en las personas mayores se asocia considerablemente con ambos síntomas de ansiedad y depresión ${ }^{280}$.

No obstante, Kempen et al. ${ }^{281}$ afirmó que la BV, la discapacidad y la falta de apoyo social contribuyen a los síntomas depresivos, y que la prevención de la discapacidad y el aumento del apoyo social pueden ayudar a reducir los síntomas de la depresión en los adultos mayores con BV. Horowitz et al.282 ratifica los hallazgos de la investigación mencionada al evaluar la influencia de la salud, la calidad del apoyo social y la rehabilitación en la depresión de 95 personas mayores con DV. Ser soltero(a) y tener menor calidad de relaciones con la familia y amigos fueron importantes factores de riesgo para la depresión en su estudio. Además, la utilización de los servicios de rehabilitación visual disminuyó la sintomatología depresiva con el tiempo en los dos años de seguimiento del estudio. El hecho de que en nuestro estudio, la mayoría de las personas con BV estuviesen casadas (61.29\%) y todas ellas frecuentasen un centro de RV podría explicar el hecho de que los mayores con BV en este estudio no presentasen en su mayoría síntomas depresivos, por lo que no hubo diferencias estadísticamente significativas entre los dos grupos.

\subsubsection{El miedo a caerse en ambos grupos}

La preocupación por caerse es común entre las personas mayores y en personas con dificultades de movilidad. En un reciente estudio con el fin de estimar la frecuencia de caídas recientes y la prevalencia del miedo a caer en adultos mayores de 65 años no-institucionalizados, más de un tercio de los 1709 participantes señalaron que tenían moderado o mucho miedo de caer ${ }^{283}$.

Además de la edad, hay recientes estudios que sugieren que la DV está asociada con un mayor miedo a caer. En una revisión de la literatura actual, nuevas evidencias indican que el miedo a caerse es más frecuente en los adultos mayores con DV que en aquellos con visión normal, sin embargo esto es algo que todavía no ha sido ampliamente investigado ${ }^{284}$. El estudio realizado por Ramulu et al. ${ }^{285}$ tuvo como objetivo comparar el miedo a caerse en 83 sujetos con glaucoma con pérdida bilateral del CV, y 60 sujetos control con buena AV y sin pérdida significativa del CV. Sus resultados apuntan a que el glaucoma tiene un impacto significativo en el miedo a caerse y que, el miedo a caerse es sustancialmente mayor con el aumento de la gravedad de la enfermedad.

Otra DV asociada al miedo a caerse es la DMAE, dónde la relación entre esta y la pérdida de visión en pacientes con edades entre 60-80 años con dicha enfermedad fue verificada. Tal estudio afirmó que la DMAE está asociada con 
un mayor miedo a caerse, e incluso que el miedo a caerse es mayor en los pacientes con peores $\mathrm{AV}$ y SC${ }^{286}$.

Tal y como afirman los estudios citados anteriormente, las personas mayores suelen tener una preocupación por caerse y si añadimos a eso la DV, dicho miedo se hace todavía más elevado. Eso podría explicar el hecho de que, en este estudio, la preocupación por caerse fue significativamente mayor en el grupo de las personas con BV, cuando se comparó con el grupo control.

\subsubsection{El dolor percibido en ambos grupos}

El dolor es descrito por Merskey y Bogduk ${ }^{287}$ como una "experiencia sensorial y emocional desagradable asociada con daño tisular real o potencial". En las personas mayores la evaluación del dolor puede ser particularmente difícil debido a los cambios relacionados con la visión, la audición y la cognición.

En la literatura hay inconsistencias sobre si el dolor aumenta o disminuye en las personas mayores. Además, determinar la prevalencia definitiva del dolor en las personas mayores se hace difícil teniendo en cuenta las diferencias sustanciales en la población, como las emociones, el contexto social y ambiental, los antecedentes socio culturales, el significado del dolor a la persona y las creencias, actitudes y expectativas, así como los factores biológicos ${ }^{288}$; las diferencias en los métodos de encuesta utilizados y la definición de los casos utilizados en los estudios publicados.

En el estudio realizado por Zetterlund et al. ${ }^{85}$ se evaluó la conexión entre las afecciones visuales y las dolencias musculares (que refiere el paciente mismo), se concluyó que es más estrecha cuando el paciente padece BV. Participaron del estudio 24 personas con DMAE con edades entre 65-85 años, y otras 24 personas sin problemas visuales que presentaban una distribución de edades similar. Los resultados de este estudio describen que existe una relación entre la BV y las quejas musculares.

No obstante, hay estudios que mencionan el importante papel de los factores biopsicosociales en la forma con que las personas refieren la experiencia del dolor en general (la intensidad del dolor, la angustia psicológica, deterioro funcional y estrategias de afrontamiento utilizadas) ${ }^{289}$. Asimismo, la influencia de las actitudes y creencias de los pacientes, de la ansiedad, la depresión y el estrés ${ }^{288,290}$. McParland y Knussen ${ }^{291}$ realizaron un estudio con 95 pacientes con más de 18 años, los cuales eran miembros de grupos de apoyo para artritis y fibromialgia, para investigar la influencia de las creencias sociales en el dolor. Sus resultados afirman que, en comparación con los adultos en edad laboral, los participantes de más edad tenían creencias más fuertes en un mundo justo en el cual cada uno obtiene lo que merece, experimentando menos dolor, discapacidad y angustia psicológica. 
Con lo cual el hecho de que, en este estudio, no se encontraron diferencias estadísticamente significativas entre el grupo caso y el control en lo que se refiere al dolor autopercibido, podría estar relacionado a la ausencia de síntomas depresivos en el grupo caso.

El dolor también fue relacionado a la postura corporal. Hay estudios que demuestran que las personas con dolor en determinadas regiones del cuerpo adoptan posturas corporales diferentes a las personas que no presentan dolor $256,292,293$. Sin embargo, teniendo en cuenta que en este estudio la mayoría de los participantes no presentaron dolor y que no hubo diferencias estadísticamente significativas para esta variable entre ambos grupos, no hay evidencias que apoyen que el dolor tuvo una influencia significativa sobre la postura con esta muestra en particular.

\subsubsection{Discusión de los resultados de la correlación entre las variables}

6.2.9.1. La VL y la Postura corporal en sedestación

Para el grupo caso, a pesar de que se encontró una relación entre la postura corporal y la VL, la misma no fue estadísticamente significativa. Lo que es contradictorio al tener en cuenta que en dicho grupo las alteraciones posturales halladas fueron más acentuadas que en el grupo control, para el cual la relación con la VL sí fue estadísticamente significativa para la mayoría de los ángulos posturales evaluados.

Los resultados obtenidos en todos los ángulos medidos en ambos grupos refieren una postura de inclinación hacia delante, resultando en una posición de mayor acercamiento al texto principalmente en el grupo caso.

El efecto de la postura corporal en la posición sentada sobre la eficacia lectora principalmente en las personas mayores es algo que, hasta nuestro conocimiento actual, no fue muy investigado. Crowe y FritzGerald ${ }^{294}$ afirman que adoptar una buena postura durante la actividad de lectura es importante para mejorar la concentración y favorece una adquisición más beneficiosa de lo que se está leyendo. Al mismo tiempo, evita problemas de salud en general principalmente a los que dedican muchas horas en dicha postura.

En un estudio realizado por Wang et al. ${ }^{295}$ se examinó la ergonomía de la lectura y el comportamiento al leer de niños en edad escolar. Dicho estudio encontró que la región cráneo-cervical de la mayoría de los niños presentó una inclinación hacia delante para leer, disminuyendo la distancia de lectura. La distancia de lectura y la inclinación de la cabeza se correlacionaron negativamente, es decir, la cabeza tendía a inclinarse más hacia delante que el libro a acercarse de los ojos y del cuerpo $(r=0.37, p<0.001)$. Además de eso, halló una correlación positiva entre la distancia de lectura y la VL, es decir que mayor distancia de lectura se asoció a mayor $V L(r=0.30, p<0.001)$. Lo que 
explicaría el hecho de que mayores valores en los ángulos FCu, ACC, AT y AL se asociaron con menor $\mathrm{VL}$ en el grupo control en nuestro estudio.

El acercamiento al texto hace con que el tamaño de la letra en la retina sea más grande conduciendo a la reducción del "visual span" 295 , entendido como el rango de letras que puede ser reconocido de forma fiable sin mover los ojos. El hecho de que el tamaño del "visual span" es un factor que posee fuerte influencia sobre la $\mathrm{VL}^{296}$ y que el mismo se ve limitado por las posturas corporales que promueven mayor acercamiento al texto, podría explicar la correlación entre la postura y la VL encontrada en dicho estudio.

El estudio de Ko et al. ${ }^{297}$ concuerda con el mencionado anteriormente, al afirmar que los cambios en la postura pueden alterar la distancia al objeto de lectura y, por lo tanto, el ángulo visual de la letra, definido como siendo la relación de la altura de la letra y la distancia de visión. Así que, cuanto mayor la inclinación del individuo hacia delante más cerca estará el mismo del texto, conduciendo a la disminución del campo de visión. Dicha restricción contribuye a la reducción de la longitud de los movimientos sacádicos y del número de caracteres reconocidos en cada momento de fijación ${ }^{298}$. Al tener en cuenta que lo que determina la capacidad lectora de una persona es el número y la duración de sus momentos de fijación y que los mismos están influenciados por la distancia de lectura, podría explicar la relación encontrada entre la postura corporal y la VL en ambos grupos.

En lo que se refiere al grupo caso, la relación encontrada entre la mayoría de los ángulos y la VL fue opuesta al del grupo control. Es decir, cuanto mayor el acercamiento al texto, mayor la VL. Teniendo en cuenta que la mayoría de los participantes del grupo caso utilizaron ayudas de magnificación ópticas para leer explicaría tal hallazgo ya que es necesario acercarse a dicha ayuda para utilizarla. $\mathrm{Y}$, el hecho de que los ángulos posturales medidos no tuvieron un efecto estadísticamente significativo sobre la VL podría ser porque las ayudas de magnificación óptica producen una disminución considerable del campo de visión $^{298}$, con lo cual esta evidencia sugiere que independientemente de la postura corporal adoptada en el momento de la lectura, el campo de visión de por sí podría haber tenido un efecto mucho mayor en la VL.

\subsubsection{La VL y el Equilibrio corporal}

El equilibrio corporal, en este estudio tuvo un efecto estadísticamente significativo sobre la VL solamente en el grupo control. No hay muchos estudios en la literatura actual sobre la influencia del equilibrio sobre la VL en personas mayores con BV. Nuestros resultados coinciden con los estudios que muestran que las dificultades de equilibrio y la VL lectura están conectadas.

La literatura actual todavía no llegó a un consenso en lo que se refiere a la relación entre el equilibrio y la VL. Algunos estudios refieren problemas de 
equilibrio en individuos con trastornos de lectura, como la dislexia, por ejemplo. Es el caso del estudio realizado por Needle et al. ${ }^{299}$ en 2006, en el cual evaluó el equilibrio en dos grupos emparejados por edad y Coeficiente de Inteligencia: 17 adultos disléxicos y 20 controles. Los resultados apuntaron a diferencias significativas en el equilibrio entre los grupos y, 24 a $82 \%$ del grupo de disléxicos mostró deterioro del equilibrio.

Un estudio realizado por Viholainen et al. ${ }^{148}$ en 2011 contradice el anterior al examinar la relación entre los problemas de equilibrio y la VL en niños con y sin un riesgo familiar de tener dislexia. Participaron del estudio 94 niños con riesgo familiar de dislexia y 85 niños sin riesgo de dislexia (grupo de comparación). Sus resultados muestran que no hay una conexión directa entre la lectura y el equilibrio, con lo cual los problemas de equilibrio por sí solos no fueron capaces de producir diferencias en las habilidades de lectura.

Otro estudio que tampoco encontró una relación estadísticamente significativa entre la VL y el equilibrio y que el perfil de los participantes era similar al del nuestro estudio fue el realizado por Radvay et al. ${ }^{149}$. Este estudio midió el impacto del entrenamiento del equilibrio en varias funciones viso-motoras y la velocidad de lectura. Para ello, evaluaron el equilibrio y el rendimiento de lectura en 55 personas mayores sin DV y, el efecto de la RV y del entrenamiento para el equilibrio en 54 personas mayores con DMAE. El criterio de inclusión para el rango de edad en ambos grupos fue de 60-87 años y para la $\mathrm{AV}$ en el grupo con DMAE fue $>0.4 \log$ MAR. De las personas sin DV, 14 fueron seleccionadas para llevar a cabo un programa de entrenamiento del equilibrio, mientras que de las personas con DMAE, 16 fueron seleccionadas para llevar a cabo el mismo programa del otro grupo, además de la RV. La mejora en la $\mathrm{VL}$ (VL antes $=27.07 \pm 11.35 \mathrm{ppm})$ no fue significativa estadísticamente después del entrenamiento del equilibrio (VL después = $31.69 \pm 14.30 \mathrm{ppm}$ ) en comparación con el programa de RV (VL después = $46.50 \pm 12.87 \mathrm{ppm})$. Dichos autores hicieron hincapié al pequeño tamaño muestral y concluyeron que más sesiones de entrenamiento podrían conducir a una mejora más significativa de las funciones viso-motoras.

El estudio realizado por Chen et al. ${ }^{300}$ podría ratificar la afirmación anterior. Ellos encontraron una relación entre el equilibrio corporal y la visión al evaluar los efectos de la práctica del Tai Chi sobre el equilibrio en personas mayores con DV. Los participantes mostraron una mejora en sus cocientes visuales y los cambios podrían deberse a una ganancia en su agudeza vestibular. Además, a pesar de tener poca o ninguna visión, las personas con DV todavía son capaces de percibir alguna entrada visual y la formación de Tai Chi, que involucra el movimiento de los ojos y de la cabeza, puede haber sido capaz de mejorar su capacidad para utilizar el resto visual y así controlar su equilibrio de manera más efectiva. 
Con lo cual, la relación encontrada entre las funciones viso-motoras y el equilibrio podrían explicar los hallazgos de este estudio.

\subsubsection{La VL y la Destreza manual}

El análisis de los resultados no mostró una correlación estadísticamente significativa entre la destreza manual y la VL. A pesar de que las variables AV y SC fueron controladas en ambos grupos por ser posibles factores que podrían afectar a la VL, solamente los participantes del grupo caso utilizaron ayudas ópticas para leer. Por lo tanto, son los resultados de dicho grupo más importantes en esta correlación.

La utilización de ayudas ópticas como las lupas, por ejemplo, restringe el campo habitual de visión (número de caracteres simultáneamente visibles) debido a las dimensiones de la lente de aumento y el nivel de ampliación, lo que hace la tarea de lectura más compleja debido a la correcta manipulación del dispositivo. Es decir, la lupa tiene que moverse para localizar la información requerida, el inicio de una nueva línea, así como para leer una línea en un texto.

Hasta nuestro conocimiento actual, el único estudio que evaluó los efectos de la destreza manual en la VL con el uso de ayudas ópticas fue el realizado por Dickinson y Shim ${ }^{145}$ en 2007. Según ellos, el análisis de los datos mostró una correlación estadísticamente significativa entre la destreza manual y la VL con la lupa. Sin embargo, no han podido discurrir sobre los motivos de las relaciones encontradas debido a la dificultad para determinar las funciones de la mano necesarias para manipular una lupa manual.

Aunque existe una considerable cantidad de tests para evaluar la destreza manual, muchos de ellos no pueden ser utilizados para personas con DV pues dependen de una buena visión para su realización. La prueba utilizada para evaluar la destreza manual en este estudio fue el Pick Up test $y$, aunque él mide diferentes aspectos de la función de la mano, varios aspectos diferentes de la función de la mano están involucrados en el uso de la lupa manual. Con lo cual, pudo haber otros aspectos igualmente relevantes de la función de la mano que no consideramos, lo que explicaría no haber encontrado una correlación estadísticamente significativa en ninguno de los grupos.

A pesar de que la destreza manual en el grupo caso fue significativamente peor que en el grupo control, su relación con la $V L$ no fue significativa estadísticamente. Considerando que la AV, la SC, el CV periférico y el tamaño del escotoma central son 4 factores que afectan significativamente la lectura ${ }^{301}$ y que la muestra participante de dicho grupo presenta problemas en todos los factores mencionados, una limitación en la destreza manual puede ser menos significativa ${ }^{145}$. Otro factor importante que podría explicar tal hallazgo es el pequeño tamaño muestral para tal grupo. 


\subsubsection{La VL y la Función visual}

Los resultados encontrados refieren una relación estadísticamente significativa entre la VL y las variables AV y SC en el grupo caso y solamente la SC en el grupo control. Es decir, cuanto peor la AV y la SC peor la VL.

La lectura es un proceso complejo que puede ser afectado por diferentes factores y la función visual es señalada como un factor de gran importancia en dicho proceso. Los estudios que miden el desempeño real de lectura han revelado una $\mathrm{VL}$ alterada en los pacientes al leer letra pequeña o textos con bajo contraste.

La disminución de la tasa de lectura para personas con DMAE está directamente relacionada con su CV, SC y la $\mathrm{AV}^{301}$. La AV proporciona una estimación de los caracteres más pequeños que se pueden leer y a menudo se asocia con una mala lectura. Sin embargo, esto no es siempre el caso pues para una AV dada, la variabilidad en las tasas de lectura es alta. Esta variabilidad, probablemente se debe en parte a las características de escotomas presentes, a la edad del sujeto y a la patología visual ${ }^{15}$.

En lo que se refiere a la SC, el grado en que las personas con glaucoma tienen dificultades con la lectura fue investigado en un estudio que midió el cambio en la $\mathrm{VL}$ al reducir el contraste del texto. Cincuenta y tres personas con glaucoma, con edad media de 66 años, y 40 sujetos control sin patología visual con edad media de 69 años participaron del estudio. Debido a la disminución del contraste del texto, la VL en los pacientes redujo una media general de $20 \%$ y dicha reducción fue significativamente más evidente en pacientes con glaucoma en comparación con las personas sin DV302.

Los estudios mencionados concuerdan con nuestros hallazgos de que la AV y la SC están relacionados con la VL principalmente en las personas mayores con $\mathrm{BV}$, y ratifican que las personas con $\mathrm{BV}$ generalmente requieren grandes caracteres para leer y que un alto contraste es especialmente importante para ellos ${ }^{303}$.

\subsubsection{La VL y la Depresión}

Los resultados analizados indican que hubo una correlación estadísticamente significativa entre la VL y la sintomatología depresiva en el grupo control. Aunque dicha correlación no fue estadísticamente significativa en el grupo caso, hay estudios que muestran que la depresión es un factor clave que puede influenciar y agravar la discapacidad ${ }^{160,304}$ y además que puede afectar negativamente la lectura contribuyendo al fracaso de dicho programa ${ }^{305}$. Por tanto, es un factor importante que hay que tener en cuenta a la hora de elaborar un programa de RV. 
La depresión en las personas mayores es un tema ampliamente investigado, principalmente cuando las mismas presentan alguna DV280,306,307. A pesar de que algunos autores han utilizado la escala GDS para relacionar la depresión con la DV, en nuestro conocimiento no se conoce hasta el momento trabajos publicados que relacionen esta escala con la VL. No obstante, Grant et al. ${ }^{308}$ investigaron la relación entre la depresión y algunas medidas cuantitativas de la función visual (agudeza, VL (ppm) y el tamaño de la letra) en 18 personas mayores con DMAE utilizando el Center for Epidemiologic Studies Depression Scale (CES-D) para evaluar la depresión y el Minnesota Low Vision Reading Acuity Chart (MNREAD) para medir la VL. Sus resultados indican que los participantes que reportaron menos depresión experimentaron una mayor mejoría en la VL después del entrenamiento. Con lo cual, los niveles de depresión pueden afectar negativamente a los resultados de la rehabilitación destinada a la lectura.

El hecho de que, en este estudio, el tamaño de la muestra del grupo caso era pequeño ha podido subestimar los efectos de la sintomatología depresiva sobre la VL.

\subsubsection{La Depresión y la Postura corporal}

Son muchos los estudios que relacionan la postura corporal al estado de ánimo. De hecho, la postura en la posición sentada fue relacionada a pensamientos negativos y a pérdida de la motivación. En un estudio realizado por Riskind ${ }^{310}$, se evaluó la relación entre la postura corporal y las sensaciones de ganar o perder. Cuando los individuos se encorvaban después de experimentar el fracaso, tal postura minimizó los sentimientos de impotencia y depresión, pero cuando lo hacían después del éxito, perdían la motivación. En otro estudio se pidió a los participantes para asignar diferentes emociones a varias posturas representadas en algunas figuras. A la figura con la postura erguida y erecta los participantes prestaron las emociones más positivas, mientras que a la figura que tenía la postura de los hombros y la cabeza hacia adelante se le dieron la emoción más negativa ${ }^{311}$.

Tras evaluar si era más fácil de generar pensamientos positivos o negativos en la postura erecta o encorvada de 24 personas, Wilson y Peper ${ }^{312}$ encontraron que el $92 \%$ de los participantes indicaron que era más fácil tener pensamientos positivos en la posición erecta, corroborando los estudios citados anteriormente. Una mayor depresión y la disminución de la motivación también fueron relacionadas según el nivel de la flexión corporal. Balzini et al. ${ }^{313}$ evaluaron la intensidad de la postura flexionada y sus características clínicas multidimensionales, entre ellas la depresión. Sus hallazgos indican que el grupo de personas con mayor flexión postural tuvo mayor depresión y presentó menor motivación. La deformación del cuerpo o frecuentes episodios de dolor podría ser la causa de la depresión en esta población. 
Según los estudios mencionados, la postura encorvada, flexionada o inclinada hacia delante está relacionada con el estado de ánimo del sujeto. En este estudio se encontró una correlación entre la posible sintomatología depresiva y el ángulo cérvico-torácico en la posición sentada, siendo este el único estadísticamente significativo. El hecho de que la mayoría de los participantes no presentó sintomatología depresiva y el tamaño de la muestra con posible sintomatología depresiva era pequeño podría justificar dichos resultados.

\subsubsection{La Depresión y la Equilibrio corporal}

Por el hecho de que hay muchos factores de riesgo que conducen a un equilibrio deteriorado y al surgimiento de la sintomatología depresiva, han sido pocos los estudios que investigaron la relación entre depresión y equilibrio de manera aislada. La literatura actual muestra una fuerte relación entre la depresión y el estado de salud y entre la depresión y la capacidad funcional. Es decir, una persona con problemas en el equilibrio corporal siente miedo al caminar y preocupación por caerse, además de la pérdida de la independencia y la disminución de la calidad de vida. En las personas mayores el miedo es todavía más presente debido deterioro de sus capacidades físicas inherentes a la edad avanzada. Con lo cual, dichas personas pierden la confianza en sí mismas, la autoestima y la motivación, empezando a aislarse de la sociedad. Todo este proceso puede provocar el surgimiento de la sintomatología depresiva.

Kwan et al. ${ }^{213}$ evaluaron la presencia o no de la sintomatología depresiva, a través del GDS, de trastornos sensoriomotores, de problemas en el equilibrio y de movilidad en 280 personas con edad media de 74.9 años. Los participantes seguidos durante 2 años con llamadas telefónicas mensuales para determinar la incidencia de caídas. Sus hallazgos apuntan a que los síntomas depresivos fueron significativamente más frecuentes en las personas que sufrieron caídas recurrentes $(40 \%)$ y que el trastorno del equilibrio fue identificado como un factor predictivo significativo de las mismas, confirmando la relación existente entre ambos. Lo que concuerda con los resultados de este estudio, en el cual se encontró una asociación estadísticamente significativa entre ambas variables. Es decir, un peor desempeño en la prueba de equilibrio fue un factor de riesgo para la probabilidad de tener sintomatología depresiva.

\subsubsection{La Depresión y la Destreza manual}

La depresión en las personas mayores se deriva de cambios en la fisiología, de la genética y de los agentes ambientales. Su relación con el estado de salud percibida y la capacidad funcional ha sido investigada por diferentes estudios. De hecho, tanto la depresión cuanto la destreza manual son factores que están asociados al deterioro funcional en las personas mayores ${ }^{314}$. 
La relación entre la destreza manual y la depresión fue encontrada en por Soyupek et al. ${ }^{315}$ al estudiar la función manual en las mujeres con síndrome de ovario poliquístico. Participaron del estudio 37 mujeres con el síndrome mencionado y 35 mujeres sanas de la misma edad. Dichos autores concluyeron que los puntajes de los parámetros de la mano y la depresión fueron altos en el grupo con el síndrome de ovario poliquístico, además de haber una correlación entre ambas variables. Del mismo modo, el riesgo de depresión aumentó especialmente en los pacientes con el síndrome que presentaron déficits en la fuerza y destreza manuales.

En un estudio realizado por Penta et al. ${ }^{316}$ la relación entre la destreza manual y la depresión también fue verificada. Ciento tres pacientes con accidente cerebrovascular crónico (62\% hombres, edad media, 63 años) participaron del estudio. Las variables evaluadas fueron la habilidad manual, la motricidad del miembro superior, la destreza manual, la sensación táctil y la depresión. Sus resultados apuntan a una relación estadísticamente significativa entre la depresión y las medidas de habilidad manual $(p=0.030)$.

Según los autores citados, la depresión y la destreza manual son dos variables que están relacionadas entre sí y dicha relación fue también encontrada en este estudio, dónde la destreza manual fue significativamente peor en el grupo que presentó más sintomatología depresiva.

\subsubsection{La Depresión y el Miedo a caerse}

La depresión clínica en los ancianos es común y a menudo dichas personas pueden presentar sus síntomas de forma diferente. Diferentes estudios investigaron sus efectos sobre algunos aspectos como la capacidad funcional y el estado de salud percibida. Entre ellos está el estudio realizado por RaccioRobak et al. ${ }^{317}$, en lo cual afirma que los adultos mayores deprimidos informan de una disminución de la independencia, aumento de problemas de salud crónicos y mala salud. Por otra parte, según certifican algunos estudios, la gravedad de la depresión está asociada con una disminución de la calidad de vida, con el deterioro funcional y la discapacidad ${ }^{318,319}$.

Dichas características se describen en la literatura como algunos de los factores de riesgo para el desarrollo del miedo a caerse ${ }^{320}$. Tras evaluar el miedo a caerse y su relación con la ansiedad, la depresión y participación en actividades entre adultos mayores, Painter et al. ${ }^{321}$ concluyeron que existen relaciones significativas entre el miedo a caerse y la depresión y, tanto la ansiedad como la depresión predijeron restricción de la actividad por miedo a caerse además de otras razones.

En un estudio realizado para evaluar el miedo a caerse en 1282 mujeres con edades entre 70-85 años, el miedo a caerse se asoció con una serie de variables incluyendo la depresión. Sus resultados apuntaron que el miedo a 
caerse en las mujeres mayores es una queja común y persistente y que la depresión en esta población puede ocurrir como un efecto de la preocupación por caerse por un prolongado periodo de tiempo ${ }^{322}$. La preocupación por caerse entre las personas de edad avanzada puede comprometer la calidad de vida mediante la limitación de la movilidad, disminución del sentido del bienestar y la reducción de las interacciones sociales. El nivel educativo, los problemas de salud asociados, historia previa de caídas, restricción de las actividades diarias y la depresión presentan una estrecha correlación con el miedo a caerse ${ }^{323}$. Con lo cual, los datos presentados por los estudios mencionados justifican los hallazgos de este estudio, en el cual la preocupación por caerse fue significativamente mayor en el grupo de las personas con sintomatología depresiva.

\subsubsection{La Depresión y el Dolor}

El dolor percibido es una experiencia compleja que puede ser afectado por un vasto número de aspectos biopsicosociales, incluyendo las emociones, el contexto social y ambiental, sociocultural, el significado del dolor a la persona, y las creencias, actitudes y expectativas, así como factores biológicos ${ }^{288,289,291,324}$. En una revisión sistemática de la literatura, realizada con el objetivo de investigar la depresión en el curso de la lumbalgia aguda y subaguda, mostró que once de los 17 artículos incluidos en ella relacionan los síntomas de depresión al inicio del estudio con peores resultados de dolor en la espalda baja durante el seguimiento. Los estudios incluidos en dicha revisión apuntan a un efecto negativo de los síntomas de la depresión sobre los resultados del dolor de espalda ${ }^{324}$.

Costa et al. ${ }^{325}$ investigó la percepción de la enfermedad como predictor de la depresión y ansiedad en pacientes con dolor crónico. Para ello, reclutó a 200 pacientes en una unidad de dolor en un hospital público en el norte de Portugal. La depresión y los síntomas de ansiedad se asociaron con la intensidad del dolor, la discapacidad relacionada con el dolor y la percepción de la enfermedad, lo que significa que la percepción de la enfermedad contribuyó significativamente a la depresión y a los síntomas de ansiedad. Igualmente, la percepción de una mayor sintomatología e impacto emocional, y menor percepción de control del tratamiento y comprensión del dolor crónico se asociaron significativamente con el aumento de los síntomas de depresión. Tales resultados confirman los resultados de un estudio sobre predictores psicosociales del dolor, de la incapacidad y de la depresión en pacientes con dolor crónico. Tal estudio sugiere que la depresión en dicha población puede estar influenciada por otros factores, además de la intensidad del dolor, la discapacidad y la autoeficacia. Además, confirman los modelos biopsicosociales del dolor en los cuales los factores biopsicosociales tienen un diferente papel sobre el dolor, la incapacidad y la depresión ${ }^{289}$. 
Los factores biopsicosociales mencionados anteriormente podrían justificar el hecho de que en este estudio no se encontró relación entre la sintomatología depresiva y el dolor autopercibido. Es decir, la forma con que las personas viven y refieren la experiencia del dolor en general tiene un papel importante en la intensidad del dolor percibido, en la angustia psicológica, en el deterioro funcional y en las estrategias de afrontamiento utilizadas.

\subsubsection{Discusión del modelo multivariante}

El análisis del modelo multivariante utilizado ratificó los resultados encontrados y discutidos, y encontró que de todas las variables que tuvieron un efecto sobre la VL, FCu, ACC, AT, AL, IP y equilibrio, las que más influyeron fueron el AT, el $A L$ y el equilibrio corporal.

Los valores del AL y AT aumentan cuanto mayor la flexión de la columna. EI acercamiento al objeto de lectura lleva a la flexión de la columna y a la disminución de la VL. Tal hallazgo es un factor preocupante pues las posturas de flexión de la columna están relacionadas con un aumento del estrés vertebral y de la presión intradiscal en el raquis torácico y lumbar. Además, pueden afectar negativamente a la propiocepción espinal y puede estar asociada con dolor en la región lumbar ${ }^{79,81,82,309}$.

Beach et al. ${ }^{81}$ confirman la relación existente entre la postura sentada y la columna lumbar después de evaluar los cambios en la rigidez de la columna lumbar en la postura sentada durante un determinando periodo de tiempo. Además, investigaron también si los hombres y las mujeres respondían de manera diferente a estar mucho tiempo sentado. Doce estudiantes universitarios (6 hombres, 6 mujeres) sin dolor lumbar reciente fueron evaluados durante 2 horas sentados en una silla de oficina. Sus resultados apuntan que la rigidez de la columna lumbar aumentó en los hombres después de sólo 1 hora sentados, mientras que las respuestas de las mujeres fueron variables durante el ensayo de 2 horas.

Womersley y May investigaron la relación entre la postura sentada y el dolor de espalda. Para ello, evaluaron la postura sentada y monitorearon las actividades posturales normales de dos grupos de jóvenes participantes (un grupo con dolor de espalda y el otro sin dolor de espalda) durante un período de 3 días. Al final del estudio, concluyeron que los síntomas de las personas con dolor de espalda están asociados con posturas sentadas sostenidas y de mayor flexión. En el grupo de estudiantes que reportó dolor de espalda postural estaban sentados por períodos de tiempo más largos sin interrupción y tenían una postura sentada más flexionada que el grupo sin dolor de espalda. 
Con lo cual, la higiene postural en el momento de lectura es importante para prevenir alteraciones posturales, dolor y fatiga muscular. Además, evita el abandono de la lectura y, consecuentemente del programa de RV. 
7. CONCLUSIONES 



\subsection{Conclusiones}

Ha sido posible concluir que:

1. La postura y el equilibrio corporal tienen una influencia estadísticamente significativa y clínicamente relevante sobre la VL, incrementándose los valores de dicha variable en las personas mayores sin BV. Sin embargo, la destreza manual no es un factor influyente sobre la $\mathrm{VL}$ en ninguno de los grupos analizados en este estudio.

2. Las personas mayores con $\mathrm{BV}$ presentan una postura de mayor flexión que las personas mayores sin BV principalmente en sedestación; asimismo su destreza manual es menos eficaz y presentan peores valores en la función visual. No obstante, no hay diferencias entre las personas mayores con y sin BV en lo que se refiere al equilibrio corporal.

3. El LUMOback como método para medir la postura corporal es un sensor fiable y válido que puede ser incorporado a la práctica clínica diaria en las consultas de fisioterapia y rehabilitación visual.

4. El método de análisis postural y demarcación de los puntos anatómicos llevados a cabo en este estudio fue considerado fiable y reproducible.

5. Los principales predictores en el aumento de la dificultad lectora en las personas mayores sin BV son los ángulos posturales del tronco (AT) y lumbar $(\mathrm{AL})$ y el equilibrio corporal.

6. La postura corporal en sedestación en su ángulo cérvico-torácico, el equilibrio corporal, la destreza manual y la VL son factores de riesgo para la presencia de la sintomatología depresiva en personas mayores con y sin BV. Las personas mayores con BV refieren más miedo a caerse que las personas mayores sin BV. Sin embargo, la sintomatología depresiva y el dolor percibido son similares en ambos grupos. 


\subsection{Limitaciones del estudio}

La escasez de estudios sobre la postura de personas mayores con BV y la heterogeneidad en los métodos de evaluación postural han dificultado enormemente la comparación de nuestros resultados y la posible generalización de los mismos. Sin embargo, teniendo en cuenta la fiabilidad del método de evaluación postural realizado en este estudio, los resultados posturales encontrados pueden contribuir para la generación de valores de referencia en estudios futuros.

El reducido tamaño de nuestra muestra, principalmente de personas con BV, podría limitar las posibilidades de generalización de los resultados. La gran pérdida de participantes entre el primer contacto y la recogida de datos demuestra la realidad de reclutamiento para estudios transversales, donde hay dificultad sustancial en lograr muestras adecuadas. Además, tener una muestra más grande habría hecho que el trabajo se hubiera prolongado excesivamente en el tiempo, por lo que no se consideró adecuado.

También hubiese sido deseable contar con diferencias entre pacientes y controles en lo que se refiere a la sintomatología depresiva, para poder estudiar con más precisión la relación entre esta variable y las variables posturales y de lectura, aunque posiblemente la ausencia de sintomatología depresiva en el grupo caso sea porque están incluidos en este grupo pacientes con diferentes grados de DV y con distintas patologías visuales.

\subsection{Continuación de esta línea de investigación}

A pesar de las limitaciones, se puede decir que en principio los datos apuntan hacia una mayor alteración de la postura corporal, equilibrio y la destreza manual en las personas mayores con BV. Por lo tanto, son necesarios estudios futuros que incrementen el tamaño de la muestra, ampliando los colectivos a niños, jóvenes y adultos con diferentes patologías visuales y niveles de DV y diferentes ayudas ópticas y diseñando programas de intervención eficaces.

\subsection{Implicaciones para la práctica clínica}

Los resultados de este estudio muestran que tanto la postura corporal cuanto el equilibrio disminuyen la eficacia de la VL. La evaluación de dichos aspectos podría ser implantada en la práctica clínica diaria en RV, los cuales deberían tener influencia a la hora de desarrollar y aplicar programas de RV. La adquisición de conocimientos sobre la ergonomía y la práctica de buenos hábitos posturales con la supervisión de un fisioterapeuta pueden hacer que el proceso de aprendizaje y la lectura con ayudas ópticas sean más agradables y menos agotadores, evitando el abandono del programa de $\mathrm{RV}$ y previniendo dolores y lesiones musculoesqueléticas. 
Además, teniendo en cuenta que el ángulo postural lumbar fue unos de los factores predictivos en el aumento de la dificultad lectora y que la fiabilidad del LUMOback fue comprobada en este estudio, dicho sensor podría ser empleado tanto para evaluar como para entrenar la postura del paciente con BV durante el entrenamiento de la lectura. 

8. REFERENCIAS 

1. Instituto de Mayores y Servicios Sociales (IMSERSO). Envejecimiento Activo. Libro Blanco. 728 p (2011).

2. Seidler, R. D. et al. Motor Control and Aging: Links to Age-Related Brain Structural, Functional, and Biochemical Effects. Neurosci. Biobehav. Rev. 34, 721-733 (2010).

3. Desrosiers, J., Hébert, R., Bravo, G. \& Rochette, A. Age-related changes in upper extremity performance of elderly people: a longitudinal study. Exp. Gerontol. 34, 393-405 (1999).

4. Falconer, J. et al. Self report and performance-based hand function tests as correlates of dependency in the elderly. J. Am. Geriatr. Soc. 39, 695699 (1991).

5. Núñez, A. R. C. et al. Evaluación geriátrica integral del adulto mayor. Rev. Médica Chile 139, 725-731 (2011).

6. Hinds, A. et al. Impact of an interdisciplinary low vision service on the quality of life of low vision patients. Br. J. Ophthalmol. 87, 1391-1396 (2003).

7. Renieri, G., Pitz, S., Pfeiffer, N., Beutel, M. E. \& Zwerenz, R. Changes in quality of life in visually impaired patients after low-vision rehabilitation. Int. J. Rehabil. Res. Int. Z. Für Rehabil. Rev. Int. Rech. Réadapt. 36, 48-55 (2013).

8. Hooper, P., Jutai, J. W., Strong, G. \& Russell-Minda, E. Age-related macular degeneration and low-vision rehabilitation: a systematic review. Can. J. Ophthalmol. J. Can. Ophtalmol. 43, 180-187 (2008).

9. Trauzettel-Klosinski, S. Current Methods of Visual Rehabilitation. Dtsch. Ärztebl. Int. 108, 871-878 (2011). 
10. Bowers, A., Cheong, A. M. \& Lovie-Kitchin, J. E. Reading with optical magnifiers: page navigation strategies and difficulties. Optom. Vis. Sci. Off. Publ. Am. Acad. Optom. 84, 9-20 (2007).

11. Burton, R., Smith, N. D. \& Crabb, D. P. Eye movements and reading in glaucoma: observations on patients with advanced visual field loss. Graefes Arch. Clin. Exp. Ophthalmol. 252, 1621-1630 (2014).

12. Nguyen, N. X., Besch, D., Bartz-Schmidt, K., Gelisken, F. \& TrauzettelKlosinski, S. Reading performance with low-vision aids and vision-related quality of life after macular translocation surgery in patients with agerelated macular degeneration. Acta Ophthalmol. Scand. 85, 877-882 (2007).

13. Nguyen, N. X., Weismann, M. \& Trauzettel-Klosinski, S. Improvement of reading speed after providing of low vision aids in patients with age-related macular degeneration. Acta Ophthalmol. (Copenh.) 87, 849-853 (2009).

14. Pearce, E., Sivaprasad, S. \& Chong, N. V. Factors Affecting Reading Speed in Patients with Diabetic Macular Edema Treated with Laser Photocoagulation. PLOS ONE 9, (2014).

15. Legge, G. E., Ross, J. A., Isenberg, L. M. \& LaMay, J. M. Psychophysics of reading. Clinical predictors of low-vision reading speed. Invest. Ophthalmol. Vis. Sci. 33, 677-687 (1992).

16. Krueger, H., Conrady, P. \& Zülch, J. Work with magnifying glasses. Ergonomics 32, 785-794 (1989).

17. O, R. H. Eye - neck/scapular area interactions during strenuous near work Biologically plausible pathways with relevance for work related 
musculoskeletal disorders of the neck and upper extremity. Z. Für Arbeitswissenschaft 62, (2008).

18. De Wall, M., Van Riel, M. P. J. M., Snijders, C. J. \& Van Wingerden, J. P. The effect on sitting posture of a desk with a $10^{\circ}$ inclination for reading and writing. Ergonomics 34, 575-584 (1991).

19. Hartwig, A., Gowen, E., Neil Charman, W. \& Radhakrishnan, H. Analysis of head position used by myopes and emmetropes when performing a nearvision reading task. Vision Res. 51, 1712-1717 (2011).

20. Pynt, J., Mackey, M. G. \& Higgs, J. Kyphosed Seated Postures: Extending Concepts of Postural Health Beyond the Office. J. Occup. Rehabil. 18, 3545 (2008).

21. Straker, L. M., O'Sullivan, P. B., Smith, A. J. \& Perry, M. C. Relationships between prolonged neck/shoulder pain and sitting spinal posture in male and female adolescents. Man. Ther. 14, 321-329 (2009).

22. Straker, L. M., O'Sullivan, P. B., Smith, A. \& Perry, M. Computer Use and Habitual Spinal Posture in Australian Adolescents. Public Health Rep. 122, 634-643 (2007).

23. Szeto, G. P. Y., Straker, L. \& Raine, S. A field comparison of neck and shoulder postures in symptomatic and asymptomatic office workers. Appl. Ergon. 33, 75-84 (2002).

24. Firth, A. Y., Machin, J. \& Watkins, C. L. Tilt and reading speed. J. Am. Assoc. Pediatr. Ophthalmol. Strabismus 11, 52-54 (2007).

25. Üstün, T. B., Chatterji, S., Kostansjek, N. \& Bickenbach, J. WHO's ICF and Functional Status Information in Health Records. Health Care Financ. Rev. 24, 77-88 (2003). 
26. ICD-9-CM international classification of diseases, ninth revision, clinical modification, sixth edition. (U.S. Dept. of Health and Human Services, Centers for Disease Control and Prevention; Centers for Medicare and Medicaid Services, 2011).

27. Dominique Meslin. Refración Práctica. (Essilor, 2008).

28. PASTOR JIMENO, JC. GUIONES DE OFTALMOLOGIA. (S.A. MCGRAWHILL / INTERAMERICANA DE ESPAÑA, 2011).

29. Thayaparan, K., Crossland, M. D. \& Rubin, G. S. Clinical assessment of two new contrast sensitivity charts. Br. J. Ophthalmol. 91, 749-752 (2007).

30. Geneviève Prèvost. Ophthalmic Optics File - Practical Low Vision. (Essilor, 2013).

31. Silverstone, B., Lang, M. A., Rosenthal, B. \& Faye, E. E. The Lighthouse Handbook on Vision Impairment and Vision Rehabilitation: Two-volume Set. (Oxford University Press, 2000).

32. Dandona, L. \& Dandona, R. Revision of visual impairment definitions in the International Statistical Classification of Diseases. BMC Med. 4, 7 (2006).

33. World Health Organization, W. International Classification of Diseases (ICD). WHO Available at: http://www.who.int/classifications/icd/en/. (Accessed: 22nd September 2014)

34. L, D. \& A, N. A method of scoring automated visual fields to determine field constriction causing blindness. Indian J. Ophthalmol. 46, 93 (1998).

35. Dandona, L. et al. Is current eye-care-policy focus almost exclusively on cataract adequate to deal with blindness in India? The Lancet 351, 13121316 (1998). 
36. Organización Mundial de la Salud \& Organización Panamericana de la Salud. Clasificación del Funcionamiento, de la Discapacidad y de la Salud. (2013). Available at:

http://www.paho.org/hq/index.php?option=com_content\&view=category\&la yout=blog\&id=2642\&ltemid=2561\&lang=es. (Accessed: 27 th May 2015)

37. Sarabia, A. \& Egea, C. Clasificación de la OMS sobre discapacidad. Bol. Real Patron. Sobre Discapac. 15-30 (2001).

38. National Eye Institute. National Plan for Eye and Vision Research. (2004).

39. Marrero, R. C. M., Rull, I. M. \& Cunillera, M. P. Biomecánica clínica de los tejidos y las articulaciones del aparato locomotor. (Elsevier España, 2005).

40. Kirby, R. F. \& Roberts, J. A. Introductory Biomechanics. (Mouvement Pubns, 1985).

41. Hamill, J. \& Knutzen, K. Biomechanical Basis of Human Movement. (Williams \& Wilkins, 1995).

42. Houglum, P. A. \& Bertoti, D. B. Brunnstrom's clinical kinesiology. (FA Davis, 2011).

43. Pope, M. H. \& Panjabi, M. Biomechanical definitions of spinal instability. Spine 10, 255-256 (1985).

44. Ocampo Fonseca, I., Saavedra, A., Consuelo, M. de la P., Ramos, S. \& Manuel, F. Cambios en la posición de las estructuras esqueléticas del complejo cráneo-cervical posterior a una cirugía ortognática. Rev. Odontológica Mex. 17, 210-220 (2013).

45. Hall, Susan. Basic Biomechanics. (2012). 
46. Amonoo-Kuofi, H. S. Morphometric changes in the heights and anteroposterior diameters of the lumbar intervertebral discs with age. J. Anat. 175, 159-168 (1991).

47. Coskun Benlidayi, I. \& Basaran, S. Comparative study of lumbosacral alignment in elderly versus young adults: data on patients with low back pain. Aging Clin. Exp. Res. 27, 297-302 (2015).

48. Takeda, N. et al. Changes in the sagittal spinal alignment of the elderly without vertebral fractures: a minimum 10-year longitudinal study. J. Orthop. Sci. Off. J. Jpn. Orthop. Assoc. 14, 748-753 (2009).

49. Drzał-Grabiec, J., Snela, S., Rykała, J., Podgórska, J. \& Banaś, A. Changes in the body posture of women occurring with age. BMC Geriatr. 13, 108 (2013).

50. Mannheimer, J. S. \& Rosenthal, R. M. Acute and chronic postural abnormalities as related to craniofacial pain and temporomandibular disorders. Dent. Clin. North Am. 35, 185-208 (1991).

51. Snell, R. S. Neuroanatomia Clínica para estudantes de Medicina. (Guanabara Koogan, 2003).

52. Kendall, F., McCreary, E. \& Provance, P. Músculos: pruebas, funciones y dolor postural. (Marban, SL, 2000).

53. Stanos, S. P., McLean, J. \& Rader, L. Physical medicine rehabilitation approach to pain. Anesthesiol. Clin. 25, 721-759, v-vi (2007).

54. Rehabilitation of the spine: a practitioner's manual. (Lippincott Williams \& Wilkins, 2007).

55. Wallden, M. The neutral spine principle. J. Bodyw. Mov. Ther. 13, 350-361 (2009). 
56. Liebenson, C. Postural correction. J. Bodyw. Mov. Ther. 12, 318-319 (2008).

57. Rosário, J. L. P. do, Nakashima, I. Y., Rizopoulos, K., Kostopoulos, D. \& Marques, A. P. Improving posture: Comparing Segmental Stretch and Muscular Chains Therapy. Clin. Chiropr. 15, 121-128 (2012).

58. McGill, S. Low back disorders: evidence-based prevention and rehabilitation. (Human Kinetics, 2007).

59. O'Sullivan PB et al. The effect of different standing and sitting postures on trunk muscle activity in a pain-free population. Spine $\mathbf{2 7}, 1238-1244$ (2002).

60. O'Sullivan, P. B. et al. Effect of different upright sitting postures on spinalpelvic curvature and trunk muscle activation in a pain-free population. Spine 31, E707-712 (2006).

61. Keegan, J. J. Alterations of the Lumbar Curve Related to Posture and Seating. J. Bone Jt. Surg. 35, 589-603 (1953).

62. Mandal, A. C. The seated man (Homo Sedens) the seated work position. Theory and practice. Appl. Ergon. 12, 19-26 (1981).

63. Solomonow, M., Zhou, B. H., Baratta, R. V., Lu, Y. \& Harris, M. Biomechanics of increased exposure to lumbar injury caused by cyclic loading: Part 1. Loss of reflexive muscular stabilization. Spine 24, 24262434 (1999).

64. Mandal, Å. C. Work-chair with Tilting Seat. Ergonomics 19, 157-164 (1976). 
65. Bouchard, D. \& Tetreault, S. The Motor Development of Sighted Children and Children with Moderate Low Vision Aged 8-13. J. Vis. Impair. Blind. 94, 564-73 (2000).

66. Allison, L. K., Painter, J. A., Emory, A., Whitehurst, P. \& Raby, A.

Participation Restriction, Not Fear of Falling, Predicts Actual Balance and Mobility Abilities in Rural Community-Dwelling Older Adults: J. Geriatr. Phys. Ther. 36, 13-23 (2013).

67. Kotecha, A. et al. Balance Control in Glaucoma. Invest. Ophthalmol. Vis. Sci. 53, 7795-7801 (2012).

68. Sanchez, H. M., Barreto, R. R., Baraúna, M. A., Canto, R. S. T. \& Morais, E. G. de. Avaliação postural de indivíduos portadores de deficiência visual através da biofotogrametria computadorizada. Fisioter Mov 21, 11-20 (2008).

69. Fjellvang, H. \& Solow, B. Craniocervical postural relations and craniofacial morphology in 30 blind subjects. Am. J. Orthod. Dentofacial Orthop. 90, 327-334 (1986).

70. Aulisa, L., Bertolini, C., Piantelli, S. \& Piazzini, D. B. Axial deviations of the spine in blind children. Ital. J. Orthop. Traumatol. 12, 85-92 (1986).

71. Catanzariti, J. F., Salomez, E., Bruandet, J. M. \& Thevenon, A. Visual deficiency and scoliosis. Spine 26, 48-52 (2001).

72. Silva, M. B., Shimano, S. G. N., Oliveira, C. C. E. S., Conti, V. \& Oliveira, N. M. L. Avaliação das alterações posturais e retrações musculares na deficiência visual: estudo de caso. Saúde Coletiva (2011). Available at: http://www.redalyc.org/articulo.oa?id=84217104004. (Accessed: 21 st July 2015) 
73. Zhang, W. [Abnormal head posture is not equal to compensatory head posture]. Zhonghua Yan Ke Za Zhi Chin. J. Ophthalmol. 49, 586-588 (2013).

74. Castillo, G. D. \& Jorge, J. L. V. de. Anatomía y fisiología del sistema nervioso central. (Fundación Univ. San Pablo, 2015).

75. Raine, S. \& Twomey, L. T. Head and shoulder posture variations in 160 asymptomatic women and men. Arch. Phys. Med. Rehabil. 78, 1215-1223 (1997).

76. Kuo, Y.-L., Tully, E. A. \& Galea, M. P. Video Analysis of Sagittal Spinal Posture in Healthy Young and Older Adults. J. Manipulative Physiol. Ther. 32, 210-215 (2009).

77. Refshauge, K., Goodsell, M. \& Lee, M. Consistency of cervical and cervicothoracic posture in standing. Aust. J. Physiother. 40, 235-240 (1994).

78. Bauman, A. et al. The Descriptive Epidemiology of Sitting: A 20-Country Comparison Using the International Physical Activity Questionnaire (IPAQ). Am. J. Prev. Med. 41, 228-235 (2011).

79. Callaghan, J. P. \& McGill, S. M. Low back joint loading and kinematics during standing and unsupported sitting. Ergonomics 44, 280-294 (2001).

80. Harrison, D. D., Harrison, S. O., Croft, A. C., Harrison, D. E. \& Troyanovich, S. J. Sitting biomechanics part I: review of the literature. J. Manipulative Physiol. Ther. 22, 594-609 (1999).

81. Beach, T. A. C., Parkinson, R. J., Stothart, J. P. \& Callaghan, J. P. Effects of prolonged sitting on the passive flexion stiffness of the in vivo lumbar spine. Spine J. 5, 145-154 (2005). 
82. Callaghan, J. P. \& Dunk, N. M. Examination of the flexion relaxation phenomenon in erector spinae muscles during short duration slumped sitting. Clin. Biomech. Bristol Avon 17, 353-360 (2002).

83. Wilder, D. G., Pope, M. H. \& Frymoyer, J. W. The biomechanics of lumbar disc herniation and the effect of overload and instability. J. Spinal Disord. 1, 16-32 (1988).

84. Minto, H. \& Butt, I. A. Low Vision Devices and Training. Community Eye Health 17, 6-7 (2004).

85. Zetterlund, C., Lundqvist, L.-O. \& Richter, H. O. The Relationship Between Low Vision and Musculoskeletal Complaints. A Case Control Study Between Age-related Macular Degeneration Patients and Age-matched Controls with Normal Vision. J. Optom. 2, 127-133 (2009).

86. Rosário, J. L. P. do. Biomechanical assessment of human posture: A literature review. J. Bodyw. Mov. Ther. 18, 368-373 (2014).

87. Duarte, M. \& Freitas, S. M. S. F. Revision of posturography based on force plate for balance evaluation. Rev. Bras. Fisioter. São Carlos São Paulo Braz. 14, 183-192 (2010).

88. Brumagne, S., Janssens, L., Janssens, E. \& Goddyn, L. Altered postural control in anticipation of postural instability in persons with recurrent low back pain. Gait Posture 28, 657-662 (2008).

89. Hewson, D. J., Singh, N. K., Snoussi, H. \& Duchene, J. Classification of elderly as fallers and non-fallers using Centre of Pressure velocity. Conf. Proc. Annu. Int. Conf. IEEE Eng. Med. Biol. Soc. IEEE Eng. Med. Biol. Soc. Annu. Conf. 2010, 3678-3681 (2010). 
90. Piirtola, M. \& Era, P. Force platform measurements as predictors of falls among older people - a review. Gerontology 52, 1-16 (2006).

91. Rasku, J., Joutsijoki, H., Pyykkö, I. \& Juhola, M. Prediction of a state of a subject on the basis of a stabilogram signal and video oculography test. Comput. Methods Programs Biomed. 108, 580-588 (2012).

92. Corriveau, H. et al. Evaluation of postural stability in elderly with diabetic neuropathy. Diabetes Care 23, 1187-1191 (2000).

93. Corriveau, H., Hébert, R., Raîche, M. \& Prince, F. Evaluation of postural stability in the elderly with stroke1. Arch. Phys. Med. Rehabil. 85, 10951101 (2004).

94. Horak, F. B., Dickstein, R. \& Peterka, R. J. Diabetic neuropathy and surface sway-referencing disrupt somatosensory information for postural stability in stance. Somatosens. Mot. Res. 19, 316-326 (2002).

95. Horak, F. B., Dimitrova, D. \& Nutt, J. G. Direction-specific postural instability in subjects with Parkinson's disease. Exp. Neurol. 193, 504-521 (2005).

96. Lafond, D., Corriveau, H. \& Prince, F. Postural Control Mechanisms During Quiet Standing in Patients With Diabetic Sensory Neuropathy. Diabetes Care 27, 173-178 (2004).

97. Laufer, Y., Schwarzmann, R., Sivan, D. \& Sprecher, E. Postural control of patients with hemiparesis: force plates measurements based on the clinical sensory organization test. Physiother. Theory Pract. 21, 163-171 (2005).

98. Schmit, J. M. et al. Deterministic center of pressure patterns characterize postural instability in Parkinson's disease. Exp. Brain Res. 168, 357-367 (2006). 
99. Meilleur, K. G. et al. Results of a two-year pilot study of clinical outcome measures in collagen $\mathrm{VI}$ - and laminin alpha2-related congenital muscular dystrophies. Neuromuscul. Disord. 25, 43-54 (2015).

100. Gadotti, I. C., Armijo-Olivo, S., Silveira, A. \& Magee, D. Reliability of the Craniocervical Posture Assessment: Visual and Angular Measurements Using Photographs and Radiographs. J. Manipulative Physiol. Ther. 36, $619-625$ (2013).

101. Grimmer-Somers, K., Milanese, S. \& Louw, Q. Measurement of cervical posture in the sagittal plane. J. Manipulative Physiol. Ther. 31, 509-517 (2008).

102. Harrison, D. E. et al. Concurrent validity of flexicurve instrument measurements: sagittal skin contour of the cervical spine compared with lateral cervical radiographic measurements. J. Manipulative Physiol. Ther. 28, 597-603 (2005).

103. Niekerk, S.-M. van, Louw, Q., Vaughan, C., Grimmer-Somers, K. \& Schreve, K. Photographic measurement of upper-body sitting posture of high school students: A reliability and validity study. BMC Musculoskelet. Disord. 9, 113 (2008).

104. An, J.-S. et al. Influence of temporomandibular joint disc displacement on craniocervical posture and hyoid bone position. Am. J. Orthod. Dentofac. Orthop. Off. Publ. Am. Assoc. Orthod. Its Const. Soc. Am. Board Orthod. 147, 72-79 (2015).

105. Cargill, S. C., Pearcy, M. \& Barry, M. D. Three-dimensional lumbar spine postures measured by magnetic resonance imaging reconstruction. Spine 32, 1242-1248 (2007). 
106. Jorgensen, M. J., Marras, W. S. \& Gupta, P. Cross-sectional area of the lumbar back muscles as a function of torso flexion. Clin. Biomech. Bristol Avon 18, 280-286 (2003).

107. Engin, M., Demirel, A., Engin, E. Z. \& Fedakar, M. Recent developments and trends in biomedical sensors. Measurement 37, 173-188 (2005).

108. lunes, D. H., Bevilaqua-Grossi, D., Oliveira, A. S., Castro, F. A. \& Salgado, H. S. Comparative analysis between visual and computerized photogrammetry postural assessment. Braz. J. Phys. Ther. 13, 308-315 (2009).

109. Ferreira, E. A. G., Duarte, M., Maldonado, E. P., Burke, T. N. \& Marques, A. P. Postural assessment software (PAS/SAPO): validation and reliabiliy. Clinics 65, 675-681 (2010).

110. Ruivo, R. M., Pezarat-Correia, P., Carita, A. I. \& Vaz, J. R. Reliability and validity of angular measures through the software for postural assessment. Postural Assessment Software. Rehabilitación 47, 223-228 (2013).

111. Souza, J. A., Pasinato, F., Basso, D., Corrêa, E. C. R. \& Silva, A. M. T. da. Biophotogrammetry: reliability of measurements obtained with a posture assessment software (SAPO). Rev. Bras. Cineantropometria Amp Desempenho Hum. 13, 299-305 (2011).

112. Krawczky, B., Pacheco, A. G. \& Mainenti, M. R. M. A Systematic Review of the Angular Values Obtained by Computerized Photogrammetry in Sagittal Plane: A Proposal for Reference Values. J. Manipulative Physiol. Ther. 37, 269-275 (2014).

113. Straker, L. et al. The impact of computer display height and desk design on 3D posture during information technology work by young adults. $J$. 
Electromyogr. Kinesiol. Off. J. Int. Soc. Electrophysiol. Kinesiol. 18, 336349 (2008).

114. Burgess-Limerick, R., Plooy, A. \& Ankrum, D. R. The effect of imposed and self-selected computer monitor height on posture and gaze angle. Clin. Biomech. 13, 584-592 (1998).

115. Mon-Williams, M., Burgess-Limerick, R., Plooy, A. \& Wann, J. Vertical gaze direction and postural adjustment: An extension of the Heuer model. J. Exp. Psychol. Appl. 5, 35-53 (1999).

116. Straker, L., Jones, K. J. \& Miller, J. A comparison of the postures assumed when using laptop computers and desktop computers. Appl. Ergon. 28, 263-268 (1997).

117. Saito, S., Miyao, M., Kondo, T., Sakakibara, H. \& Toyoshima, H. Ergonomic Evaluation of Working Posture of VDT Operation Using Personal Computer with Flat Panel Display. Ind. Health 35, 264-270 (1997).

118. Kietrys, D. M., McClure, P. W. \& Fitzgerald, G. K. The Relationship Between Head and Neck Posture and VDT Screen Height in Keyboard Operators. Phys. Ther. 78, 395-403 (1998).

119. O'Sullivan, P. B., Smith, A. J., Beales, D. J. \& Straker, L. M. Association of Biopsychosocial Factors With Degree of Slump in Sitting Posture and SelfReport of Back Pain in Adolescents: A Cross-Sectional Study. Phys. Ther. 91, 470-483 (2011).

120. Knudson, D. Fundamentals of Biomechanics. (Springer, 2007).

121. Yim-Chiplis, P. K. \& Talbot, L. A. Defining and Measuring Balance in Adults. Biol. Res. Nurs. 1, 321-331 (2000). 
122. Horak, F. B. \& Nashner, L. M. Central programming of postural movements: adaptation to altered support-surface configurations. J. Neurophysiol. 55, 1369-1381 (1986).

123. Amiridis, I. G., Hatzitaki, V. \& Arabatzi, F. Age-induced modifications of static postural control in humans. Neurosci. Lett. 350, 137-140 (2003).

124. Horak, F. B., Nashner, L. M. \& Diener, H. C. Postural strategies associated with somatosensory and vestibular loss. Exp. Brain Res. 82, 167-177 (1990).

125. Hong, S.-W. et al. Control of body's center of mass motion relative to center of pressure during uphill walking in the elderly. Gait Posture (2015). doi:10.1016/j.gaitpost.2015.08.007

126. Kasahara, S., Saito, H., Anjiki, T. \& Osanai, H. The effect of aging on vertical postural control during the forward and backward shift of the center of pressure. Gait Posture (2015). doi:10.1016/j.gaitpost.2015.07.056

127. Manchester, D., Woollacott, M., Zederbauer-Hylton, N. \& Marin, O. Visual, vestibular and somatosensory contributions to balance control in the older adult. J. Gerontol. 44, M118-127 (1989).

128. Chen, E. W., Fu, A. S. N., Chan, K. M. \& Tsang, W. W. N. Balance control in very old adults with and without visual impairment. Eur. J. Appl. Physiol. 112, 1631-1636 (2012).

129. Lee, H. K. M. \& Scudds, R. J. Comparison of balance in older people with and without visual impairment. Age Ageing 32, 643-649 (2003).

130. Ivers, R. Q., Norton, R., Cumming, R. G., Butler, M. \& Campbell, A. J. Visual Impairment and Hip Fracture. Am. J. Epidemiol. 152, 633-639 (2000). 
131. Langley, F. \& Mackintosh, S. F. H. Functional balance assessment of older community dwelling adults: A systematic review of the literature. (2007).

132. Vega, F. C. Psicología de la lectura. (WK Educación, 2008).

133. Rayner, K. Eye Movements in Reading: Perceptual and Language Processes. (Elsevier, 2012).

134. Gough, P. B. One Second of Reading. Visible Lang. (1972).

135. Scherlen, A.-C. \& Gautier, V. The difference between mean saccade size and scotoma size is a predictive factor for reading speed. Int. Congr. Ser. 1282, 704-708 (2005).

136. Allport, D. A. \& Funnell, E. Components of the Mental Lexicon. Philos. Trans. R. Soc. Lond. B Biol. Sci. 295, 397-410 (1981).

137. Berndt, R. S. \& Caramazza, A. Semantic operations deficits in sentence comprehension. Psychol. Res. 41, 169-177 (1980).

138. LaBerge, D. \& Samuels, S. J. Toward a theory of automatic information processing in reading. Cognit. Psychol. 6, 293-323 (1974).

139. Hahn, G. A. et al. New standardised texts for assessing reading performance in four European languages. Br. J. Ophthalmol. 90, 480-484 (2006).

140. Altpeter, E. K., Marx, T., Nguyen, N. X., Naumann, A. \& TrauzettelKlosinski, S. Measurement of reading speed with standardized texts: a comparison of single sentences and paragraphs. Graefes Arch. Clin. Exp. Ophthalmol. Albrecht Von Graefes Arch. Für Klin. Exp. Ophthalmol. 253, 1369-1375 (2015).

141. Radner, W. \& Diendorfer, G. English sentence optotypes for measuring reading acuity and speed--the English version of the Radner Reading 
Charts. Graefes Arch. Clin. Exp. Ophthalmol. Albrecht Von Graefes Arch. Für Klin. Exp. Ophthalmol. 252, 1297-1303 (2014).

142. Watson, G. R., Maino, J. \& De l'Aune, W. Comparison of low-vision reading with spectacle-mounted magnifiers. J. Rehabil. Res. Dev. 42, 459 (2005).

143. Rubin, G. S. \& Feely, M. Evaluation of a reading rehabilitation programme for patients with age-related macular degeneration (AMD-READ Project). Int. Congr. Ser. 1282, 699-703 (2005).

144. Crossland, M. D. \& Rubin, G. S. Eye movements and reading in macular disease: Further support for the shrinking perceptual span hypothesis. Vision Res. 46, 590-597 (2006).

145. Dickinson, C. \& Shim, M. The Influence of Manual Dexterity on Reading Speed with a Hand-held Magnifier. Invest. Ophthalmol. Vis. Sci. 48, 43684374 (2007).

146. Watson, G. \& Berg, R. V. Near training techniques. (American Foundation for the Blind, 1983).

147. Perrin, J.-L., Paillé, D. \& Baccino, T. Reading tilted: Does the use of tablets impact performance? An oculometric study. Comput. Hum. Behav. 39, 339-345 (2014).

148. Viholainen, H. et al. Are balance problems connected to reading speed or the familial risk of dyslexia? Dev. Med. Child Neurol. 53, 350-353 (2011).

149. Radvay, X., Duhoux, S., Koenig-Supiot, F. \& Vital-Durand, F. Balance training and visual rehabilitation of age-related macular degeneration patients. J. Vestib. Res. 17, 183-193 (2007). 
150. Backman, C., Gibson, S. C. D. \& Parsons, J. Assessment of Hand Function: The Relationship between Pegboard Dexterity and Applied Dexterity. Can. J. Occup. Ther. 59, 208-213 (1992).

151. Gallus, J. \& Mathiowetz, V. Test-Retest Reliability of the Purdue Pegboard for Persons With Multiple Sclerosis. Am. J. Occup. Ther. 57, 108-111 (2003).

152. Rudman, D. \& Hannah, S. An instrument evaluation framework:

Description and application to assessments of hand function. J. Hand Ther. 11, 266-277 (1998).

153. Amirjani, N., Ashworth, N. L., Gordon, T., Edwards, D. C. \& Chan, K. M. Normative values and the effects of age, gender, and handedness on the Moberg Pick-Up Test. Muscle Nerve 35, 788-792 (2007).

154. Cheng, K. C., McKay, S. M., King, E. C. \& Maki, B. E. Does Aging Impair the Capacity to Use Stored Visuospatial Information or Online Visual Control to Guide Reach-to-Grasp Reactions Evoked by Unpredictable Balance Perturbation? J. Gerontol. A. Biol. Sci. Med. Sci. 67, 1238-1245 (2012).

155. Cheng, K. C., McKay, S. M., King, E. C. \& Maki, B. E. Reaching to recover balance in unpredictable circumstances: is online visual control of the reach-to-grasp reaction necessary or sufficient? Exp. Brain Res. 218, 589599 (2012).

156. Song, C.-S. Relationship between visuo-perceptual function and manual dexterity in community-dwelling older adults. J. Phys. Ther. Sci. 27, 18711874 (2015). 
157. Hackel, M. E., Wolfe, G. A., Bang, S. M. \& Canfield, J. S. Changes in Hand Function in the Aging Adult as Determined by the Jebsen Test of Hand Function. Phys. Ther. 72, 373-377 (1992).

158. Brown, R. L. \& Barrett, A. E. Visual impairment and quality of life among older adults: an examination of explanations for the relationship. $J$. Gerontol. B. Psychol. Sci. Soc. Sci. 66, 364-373 (2011).

159. Tabrett, D. R. \& Latham, K. Factors Influencing Self-reported VisionRelated Activity Limitation in the Visually Impaired. Invest. Ophthalmol. Vis. Sci. 52, 5293-5302 (2011).

160. Rovner BW, Casten RJ \& Tasman WS. Effect of depression on vision function in age-related macular degeneration. Arch. Ophthalmol. 120, 1041-1044 (2002).

161. Williams RA, Brody BL, Thomas RG, Kaplan RM \& Brown SI. The psychosocial impact of macular degeneration. Arch. Ophthalmol. 116, 514520 (1998).

162. Mitchell, J., Bradley, P., Anderson, S. J., Ffytche, T. \& Bradley, C. Perceived quality of health care in macular disease: a survey of members of the Macular Disease Society. Br. J. Ophthalmol. 86, 777-781 (2002).

163. Weaver Moore, L. \& Miller, M. Older men's experiences of living with severe visual impairment. J. Adv. Nurs. 43, 10-18 (2003).

164. Wong, E. Y. H., Guymer, R. H., Hassell, J. B. \& Keeffe, J. E. The Experience of Age-Related Macular Degeneration. J. Vis. Impair. Blind. 98, 629-640 (2004). 
165. Burmedi, D., Becker, S., Heyl, V., Wahl, H.-W. \& Himmelsbach, I. Emotional and social consequences of age-related low vision. Vis. Impair. Res. 4, 47-71 (2002).

166. Thurston, M., Thurston, A. \& McLeod, J. Socio-emotional effects of the transition from sight to blindness. Br. J. Vis. Impair. 28, 90-112 (2010).

167. Dogan, S. \& Erturk, N. The effect of vision on craniocervical posture and its relation to craniofacial and dentoalveolar morphology. Quintessence Int. Berl. Ger. 1985 21, 401-406 (1990).

168. Lamoreux, E. L. et al. Visual Impairment, Causes of Vision Loss, and Falls: The Singapore Malay Eye Study. Invest. Ophthalmol. Vis. Sci. 49, 528-533 (2008).

169. Lord, S. R. Visual risk factors for falls in older people. Age Ageing 35, ii42ii45 (2006).

170. Patino, C. M. et al. Central and Peripheral Visual Impairment and the Risk of Falls and Falls with Injury. Ophthalmology 117, 199 (2010).

171. Szabo, S. M., Janssen, P. A., Khan, K., Potter, M. J. \& Lord, S. R. Older Women with Age-Related Macular Degeneration Have a Greater Risk of Falls: A Physiological Profile Assessment Study. J. Am. Geriatr. Soc. 56, 800-807 (2008).

172. Tobis, J. S. et al. Falling among the sensorially impaired elderly. Arch. Phys. Med. Rehabil. 71, 144-147 (1990).

173. Tinetti, M. E. \& Powell, L. Fear of falling and low self-efficacy: a case of dependence in elderly persons. J. Gerontol. 48 Spec No, 35-38 (1993). 
174. Deshpande, N. et al. Activity Restriction Induced by Fear of Falling and Objective and Subjective Measures of Physical Function: A Prospective Cohort Study. J. Am. Geriatr. Soc. 56, 615-620 (2008).

175. Kressig, R. W. et al. Associations of Demographic, Functional, and Behavioral Characteristics with Activity-Related Fear of Falling Among Older Adults Transitioning to Frailty. J. Am. Geriatr. Soc. 49, 1456-1462 (2001).

176. Mann, R., Birks, Y., Hall, J., Torgerson, D. \& Watt, I. Exploring the relationship between fear of falling and neuroticism: a cross-sectional study in community-dwelling women over 70. Age Ageing 35, 143-147 (2006).

177. Baraúna, M. A. et al. Estudo correlacional entre lombalgia e concavidade lombar em universitários. Fisioter Bras 7, 172-176 (2006).

178. Patel, K. V., Guralnik, J. M., Dansie, E. J. \& Turk, D. C. Prevalence and Impact of Pain among Older Adults in the United States: Findings from the 2011 National Health and Aging Trends Study. Pain 154, (2013).

179. Vlaeyen, J. W., Kole-Snijders, A. M., Boeren, R. G. \& van Eek, H. Fear of movement/(re)injury in chronic low back pain and its relation to behavioral performance. Pain 62, 363-372 (1995).

180. Patel, K. V., Dansie, E. J. \& Turk, D. C. Impact of chronic musculoskeletal pain on objectively measured daily physical activity: a review of current findings. Pain Manag. 3, 467-474 (2013).

181. Lihavainen, K. et al. Contribution of Musculoskeletal Pain to Postural Balance in Community-Dwelling People Aged 75 Years and Older. J. Gerontol. A. Biol. Sci. Med. Sci. 65A, 990-996 (2010). 
182. Trauzettel-Klosinski, S. \& Dietz, K. Standardized Assessment of Reading Performance: The New International Reading Speed Texts IReST. Invest. Ophthalmol. Vis. Sci. 53, 5452-5461 (2012).

183. Podsiadlo, D. \& Richardson, S. The timed 'Up \& Go': a test of basic functional mobility for frail elderly persons. J. Am. Geriatr. Soc. 39, 142-8 (1991).

184. Moberg, E. Objective Methods for Determining the Functional Value of Sensibility in the Hand. J. Bone Joint Surg. Br. 40-B, 454-476 (1958). 185. Lobo, A., Escobar, V., Ezquerra, J. \& Seva Díaz, A. 'El Mini-Examen Cognoscitivo' (Un test sencillo, práctico, para detectar alteraciones intelectuales en pacientes psiquiátricos). [The 'Mini-Examen Cognoscitiuo': A simple and practical test to detect intellectual dysfunctions in psychiatric patients.]. Rev. Psiquiatr. Psicol. Médica 14, 39-57 (1980).

186. Hayes, M. H. \& Patterson, D. G. Experimental development of the graphic rating method. Psychol Bull 18, 98-9 (1921).

187. Ferreira, E. A. G. Posture and postural control: development and application of a quantitative method for postural evaluation. (Universidade de São Paulo, 2006).

188. Farias, N. C. et al. Avaliação postural em hemiparéticos por meio do software SAPo - Relato de caso. ConScientiae Saúde (2009). Available at: http://www.redalyc.org/resumen.oa?id=92912706016. (Accessed: 16 th September 2014)

189. Mathias, S., Nayak, U. S. \& Isaacs, B. Balance in elderly patients: the 'getup and go' test. Arch. Phys. Med. Rehabil. 67, 387-389 (1986). 
190. Bohannon, R. W. Reference values for the timed up and go test: a descriptive meta-analysis. J. Geriatr. Phys. Ther. 2001 29, 64-68 (2006).

191. Folstein, M. F., Folstein, S. E. \& McHugh, P. R. 'Mini-mental state'. J. Psychiatr. Res. 12, 189-198 (1975).

192. López Miquel, J. \& Martí Agustí, G. Mini-Examen Cognoscitivo (MEC). Rev. Esp. Med. Leg. 37, 122-127 (2011).

193. Yesavage, J. A. Y. \& Sheikh, J. I. Geriatric Depression Scale (GDS): recent evidence and development of a shorter version. Clin. Gerontol. 5, 165-173 (1986).

194. Martínez, J. et al. Versión española del cuestionario de Yesavage abreviado (GDS) para el despistaje de depresión en mayores de 65 años: adaptación y validación. Medifam 12, 26-40 (2002).

195. Yardley, L. et al. Development and initial validation of the Falls Efficacy Scale-International (FES-I). Age Ageing 34, 614-619 (2005).

196. Lomas-Vega, R., Hita-Contreras, F., Mendoza, N. \& Martínez-Amat, A. Cross-cultural adaptation and validation of the Falls Efficacy Scale International in Spanish postmenopausal women. Menopause N. Y. N19, 904-908 (2012).

197. Kersten, P., Küçükdeveci, A. \& Tennant, A. The use of the Visual Analogue Scale (VAS) in rehabilitation outcomes. J. Rehabil. Med. 44, 609-610 (2012).

198. O'Sullivan, P. et al. Lumbopelvic kinematics and trunk muscle activity during sitting on stable and unstable surfaces. J. Orthop. Sports Phys. Ther. 36, 19-25 (2006). 
199. O'Sullivan, P. B., Mitchell, T., Bulich, P., Waller, R. \& Holte, J. The relationship between posture and back muscle endurance in industrial workers with flexion-related low back pain. Man. Ther. 11, 264-271 (2006).

200. R Core Team. R: A language and environment for statistical computing (Version 3.1.3). (2015).

201. Bland, J. M. \& Altman, D. G. Statistical methods for assessing agreement between two methods of clinical measurement. Lancet Lond. Engl. 1, 307310 (1986).

202. Black, A. A., Wood, J. M., Lovie-Kitchin, J. E. \& Newman, B. M. Visual impairment and postural sway among older adults with glaucoma. Optom. Vis. Sci. Off. Publ. Am. Acad. Optom. 85, 489-497 (2008).

203. Daien, V. et al. Visual acuity thresholds associated with activity limitations in the elderly. The Pathologies Oculaires Liées à l'Age study. Acta Ophthalmol. (Copenh.) n/a-n/a (2014). doi:10.1111/aos.12335

204. Lamoureux, E. et al. The relationship between visual function, duration and main causes of vision loss and falls in older people with low vision. Graefes Arch. Clin. Exp. Ophthalmol. 248, 527-533 (2010).

205. Chew, F. L. M., Yong, C.-K., Ayu, S. M. \& Tajunisah, I. The association between various visual function tests and low fragility hip fractures among the elderly: a Malaysian experience. Age Ageing 39, 239-245 (2010). 206. Styliadis, C., Kartsidis, P., Paraskevopoulos, E., loannides, A. A. \& Bamidis, P. D. Neuroplastic Effects of Combined Computerized Physical and Cognitive Training in Elderly Individuals at Risk for Dementia: An eLORETA Controlled Study on Resting States. Neural Plast. 2015, (2015). 
207. Tsai, P.-H. et al. A Novel Application of Multiscale Entropy in Electroencephalography to Predict the Efficacy of Acetylcholinesterase Inhibitor in Alzheimer's Disease. Comput. Math. Methods Med. 2015, (2015).

208. Silva, M., Andrea, C., Orellana, R., Antonio, P. \& Marzuca-Nassr, G. N. Functional geriatric assessment in primary health care. Rev. Médica Chile 143, 612-618 (2015).

209. González-Forteza, C., Echeagaray, W., A, F. \& Jiménez Tapia, A. Escala de Depresión del Centro de Estudios Epidemiológicos (CES-D) en México: análisis bibliométrico. Salud Ment. 35, 13-20 (2012).

210. Luis Alfonso Díaz, C. A. Propuesta de una versión abreviada de la escala de Zung para depresión. Colomb. Médica (2005).

211. Melipillán Araneda, R., Cova Solar, F., Rincón González, P. \& Valdivia Peralta, M. Propiedades Psicométricas del Inventario de Depresión de Beck-II en Adolescentes Chilenos. Ter. Psicológica 26, 59-69 (2008).

212. Birk, T. et al. Development and Pilot Evaluation of a Psychosocial Intervention Program for Patients With Age-Related Macular Degeneration. The Gerontologist 44, 836-843 (2004).

213. Kwan, M. M.-S., Lin, S.-I., Close, J. C. T. \& Lord, S. R. Depressive symptoms in addition to visual impairment, reduced strength and poor balance predict falls in older Taiwanese people. Age Ageing 41, 606-612 (2012).

214. Lachman, M. E. et al. Fear of Falling and Activity Restriction: The Survey of Activities and Fear of Falling in the Elderly (SAFE). J. Gerontol. B. Psychol. Sci. Soc. Sci. 53B, P43-P50 (1998). 
215. Giné-Garriga, M., Guerra, M. \& Unnithan, V. B. The effect of functional circuit training on self-reported fear of falling and health status in a group of physically frail older individuals: a randomized controlled trial. Aging Clin. Exp. Res. 25, 329-336 (2013).

216. Lusardi, M. M. \& Smith, E. V. Development of a scale to assess concern about falling and applications to treatment programs. J. Outcome Meas. 1, 34-55 (1997).

217. Kempen, G. I. J. M. et al. The Short FES-I: a shortened version of the falls efficacy scale-international to assess fear of falling. Age Ageing 37, 45-50 (2008).

218. Delbaere, K. et al. The Falls Efficacy Scale International (FES-I). A comprehensive longitudinal validation study. Age Ageing 39, 210-216 (2010).

219. Kempen, G. I. J. M. et al. Cross-cultural validation of the Falls Efficacy Scale International (FES-I) in older people: Results from Germany, the Netherlands and the UK were satisfactory. Disabil. Rehabil. 29, 155-162 (2007).

220. Price, D. D., McGrath, P. A., Rafii, A. \& Buckingham, B. The validation of visual analogue scales as ratio scale measures for chronic and experimental pain. Pain 17, 45-56 (1983).

221. Kwan, M. M.-S., Lin, S.-I., Chen, C.-H., Close, J. C. T. \& Lord, S. R. Sensorimotor function, balance abilities and pain influence Timed Up and Go performance in older community-living people. Aging Clin. Exp. Res. 23, 196-201 (2011). 
222. Beauchet, O. et al. Timed up and go test and risk of falls in older adults: A systematic review. J. Nutr. Health Aging 15, 933-938 (2011).

223. Shimada, H., Uchiyama, Y. \& Kakurai, S. Specific effects of balance and gait exercises on physical function among the frail elderly. Clin. Rehabil. $17,472-479$ (2003).

224. Ng, C. L., Ho, D. D. \& Chow, S. P. The Moberg pickup test: results of testing with a standard protocol. J. Hand Ther. Off. J. Am. Soc. Hand Ther. 12, 309-312 (1999).

225. Yancosek, K. E. \& Howell, D. A Narrative Review of Dexterity Assessments. J. Hand Ther. 22, 258-270 (2009).

226. Steins, D., Sheret, I., Dawes, H., Esser, P. \& Collett, J. A smart device inertial-sensing method for gait analysis. J. Biomech. 47, 3780-3785 (2014).

227. Heebner, N. R., Akins, J. S., Lephart, S. M. \& Sell, T. C. Reliability and validity of an accelerometry based measure of static and dynamic postural stability in healthy and active individuals. Gait Posture (2014). doi:10.1016/j.gaitpost.2014.12.009

228. Wong, W. Y. \& Wong, M. S. Detecting spinal posture change in sitting positions with tri-axial accelerometers. Gait Posture 27, 168-171 (2008).

229. Brussee, T., van Nispen, R. M. A. \& van Rens, G. H. M. B. Measurement properties of continuous text reading performance tests. Ophthalmic Physiol. Opt. J. Br. Coll. Ophthalmic Opt. Optom. 34, 636-657 (2014). 230. Glimne, S., Brautaset, R. L. \& Seimyr, G. Ö. The effect of glare on eye movements when reading. Work Read. Mass 50, 213-220 (2015). 
231. Ousler, G. W. et al. Optimizing Reading Tests for Dry Eye Disease. Cornea 34, 917-921 (2015).

232. O'Connor, K. Gender and Women's Leadership: A Reference Handbook. (SAGE Publications, 2010).

233. Gipson, I. K. \& Turner, V. M. ¿Son las mujeres más propensas que los hombres a manifestar disfunciones visuales y ceguera? Arch. Soc. Esp. Oftalmol. 80, 325-326 (2005).

234. INE. Encuesta Europea de Salud en España 2009. (2010).

235. Llaurens, V., Raymond, M. \& Faurie, C. Why are some people left-handed? An evolutionary perspective. Philos. Trans. R. Soc. Lond. B Biol. Sci. 364, 881-894 (2009).

236. Abrams, D. M. \& Panaggio, M. J. A model balancing cooperation and competition can explain our right-handed world and the dominance of lefthanded athletes. J. R. Soc. Interface rsif20120211 (2012). doi:10.1098/rsif.2012.0211

237. Frayer, D. W. et al. More than 500,000 years of right-handedness in Europe. Laterality Asymmetries Body Brain Cogn. 17, 51-69 (2012). 238. Haymes, S. A. \& Chen, J. Reliability and validity of the Melbourne Edge Test and High/Low Contrast Visual Acuity chart. Optom. Vis. Sci. Off. Publ. Am. Acad. Optom. 81, 308-316 (2004).

239. Izatt, M. T., Bateman, G. R. \& Adam, C. J. Evaluation of the iPhone with an acrylic sleeve versus the Scoliometer for rib hump measurement in. Scoliosis 7, 14 (2012). 
240. Intolo, P., Carman, A. B., Milosavljevic, S., Abbott, J. H. \& Baxter, G. D. The Spineangel@: Examining the validity and reliability of a novel clinical device for monitoring trunk motion. Man. Ther. 15, 160-166 (2010).

241. Balg, F., Juteau, M., Theoret, C., Svotelis, A. \& Grenier, G. Validity and reliability of the iPhone to measure rib hump in scoliosis. J. Pediatr. Orthop. 34, 774-779 (2014).

242. Pereira, L. C., Rwakabayiza, S., Lécureux, E. \& Jolles, B. M. Clinical reliability of a knee smartphone application goniometer. in (2014).

243. Ng, J. K., Kippers, V., Richardson, C. A. \& Parnianpour, M. Range of motion and lordosis of the lumbar spine: reliability of measurement and normative values. Spine 26, 53-60 (2001).

244. Kooiman, T. J. M. et al. Reliability and validity of ten consumer activity trackers. BMC Sports Sci. Med. Rehabil. 7, (2015).

245. O’Sullivan, K., O’Sullivan, L., Campbell, A., O’Sullivan, P. \& Dankaerts, W. Towards monitoring lumbo-pelvic posture in real-life situations: Concurrent validity of a novel posture monitor and a traditional laboratory-based motion analysis system. Man. Ther. 17, 77-83 (2012).

246. Oh, B.-L., Kim, J., Kim, J., Hwang, J.-M. \& Lee, J. Validity and reliability of head posture measurement using Microsoft Kinect. Br. J. Ophthalmol. 98, 1560-1564 (2014).

247. Gadotti, I. C. \& Magee, D. Validity of surface markers placement on the cervical spine for craniocervical posture assessment. Man. Ther. 18, 243247 (2013).

248. Mörl, F. \& Blickhan, R. Three-dimensional relation of skin markers to lumbar vertebrae of healthy subjects in different postures measured by 
open MRI. Eur. Spine J. Off. Publ. Eur. Spine Soc. Eur. Spinal Deform. Soc. Eur. Sect. Cerv. Spine Res. Soc. 15, 742-751 (2006).

249. Humphreys, B. K., Delahaye, M. \& Peterson, C. K. An investigation into the validity of cervical spine motion palpation using subjects with congenital block vertebrae as a 'gold standard'. BMC Musculoskelet. Disord. 5, 19 (2004).

250. Hollerwöger, D. Methodological quality and outcomes of studies addressing manual cervical spine examinations: a review. Man. Ther. 11, 93-98 (2006).

251. Salvia, P. et al. Precision of shoulder anatomical landmark calibration by two approaches: a CAST-like protocol and a new anatomical palpator method. Gait Posture 29, 587-591 (2009).

252. Vincent, W. J. Statistics in Kinesiology. (Human Kinetics, 2005).

253. Fleiss, J. L. The Design and Analysis of Clinical Experiments. (Wiley, 1986).

254. Penha, P. J., Baldini, M. \& João, S. M. A. Spinal Postural Alignment Variance According to Sex and Age in 7- and 8-Year-Old Children. J. Manipulative Physiol. Ther. 32, 154-159 (2009).

255. Korakakis, V., Sideris, V. \& Giakas, G. Sitting bodily configuration: A study investigating the intra-tester reliability of positioning subjects into a predetermined sitting posture. Man. Ther. 19, 197-202 (2014).

256. Lau, K. T. et al. Relationships between sagittal postures of thoracic and cervical spine, presence of neck pain, neck pain severity and disability. Man. Ther. 15, 457-462 (2010). 
257. Lau, H. M. C., Wing Chiu, T. T. \& Lam, T.-H. The effectiveness of thoracic manipulation on patients with chronic mechanical neck pain - a randomized controlled trial. Man. Ther. 16, 141-147 (2011).

258. Black, K. M. M., McClure, P. M. \& Polansky, M. S. The Influence of Different Sitting Positions on Cervical and Lumbar Posture. [Miscellaneous Article]. Spine January 11996 21, 65-70 (1996).

259. Alricsson, M. \& Werner, S. Young elite cross-country skiers and low back pain-A 5-year study. Phys. Ther. Sport Off. J. Assoc. Chart. Physiother. Sports Med. 7, 181-184 (2006).

260. Rodríguez, J. M. M., Miñarro, P. A. L. \& Cárceles, F. A. Valoración del raquis torácico, lumbar e inclinación pélvica en ciclistas de categoría élite y máster 30. Apunts Educ. Física Deport. 17-25 (2012).

261. Uysal, S. A., Erden, Z., Akbayrak, T. \& Demirtürk, F. Comparison of balance and gait in visually or hearing impaired children. Percept. Mot. Skills 111, 71-80 (2010).

262. Maeda, A., Nakamura, K., Otomo, A., Higuchi, S. \& Motohashi, Y. Body support effect on standing balance in the visually impaired elderly. Arch. Phys. Med. Rehabil. 79, 994-997 (1998).

263. Schmid, M., Nardone, A., Nunzio, A. M. D., Schmid, M. \& Schieppati, M. Equilibrium during static and dynamic tasks in blind subjects: no evidence of cross-modal plasticity. Brain 130, 2097-2107 (2007).

264. Tomomitsu, M. S. V., Alonso, A. C., Morimoto, E., Bobbio, T. G. \& Greve, J. M. D. Static and dynamic postural control in low-vision and normal-vision adults. Clinics 68, 517-521 (2013). 
265. Willis JR, Vitale SE, Agrawal Y \& Ramulu PY. VIsual impairment, uncorrected refractive error, and objectively measured balance in the united states. JAMA Ophthalmol. 131, 1049-1056 (2013).

266. Montero-Odasso, M. et al. Gait Velocity as a Single Predictor of Adverse Events in Healthy Seniors Aged 75 Years and Older. J. Gerontol. A. Biol. Sci. Med. Sci. 60, 1304-1309 (2005).

267. Bischoff, H. A. et al. Identifying a cut-off point for normal mobility: a comparison of the timed 'up and go' test in community-dwelling and institutionalised elderly women. Age Ageing 32, 315-320 (2003).

268. Kovács, É. et al. Effects of exercise programs on balance in older women with age-related visual problems: A pilot study. Arch. Gerontol. Geriatr. 55, 446-452 (2012).

269. Cheung, K. K. W., Au, K. Y., Lam, W. W. S. \& Jones, A. Y. M. Effects of a Structured Exercise Programme on Functional Balance in Visually Impaired Elderly Living in a Residential Setting. Hong Kong Physiother. J. 26, 45-50 (2008).

270. Amirjani, N., Ashworth, N. L., Olson, J. L., Morhart, M. \& Ming Chan, K. Discriminative validity and test-retest reliability of the Dellon-modified Moberg pick-up test in carpal tunnel syndrome patients. J. Peripher. Nerv. Syst. 16, 51-58 (2011).

271. Webber, A. L., Wood, J. M., Gole, G. A. \& Brown, B. The effect of amblyopia on fine motor skills in children. Invest. Ophthalmol. Vis. Sci. 49, 594-603 (2008). 
272. Rubin, G. S. \& Feely, M. The Role of Eye Movements During Reading in Patients with Age-Related Macular Degeneration (AMD). NeuroOphthalmol. 33, 120-126 (2009).

273. Bourne, R. R. A. et al. Prevalence and causes of vision loss in high-income countries and in Eastern and Central Europe: 1990-2010. Br. J. Ophthalmol. 98, 629-638 (2014).

274. Pascolini, D. \& Mariotti, S. P. Global estimates of visual impairment: 2010. Br. J. Ophthalmol. 96, 614-618 (2012).

275. Perlmutter, M. S., Bhorade, A., Gordon, M., Hollingsworth, H. H. \& Baum, M. C. Cognitive, Visual, Auditory, and Emotional Factors That Affect Participation in Older Adults. Am. J. Occup. Ther. 64, 570-579 (2010). 276. Virgili, G., Acosta, R., Grover, L. L., Bentley, S. A. \& Giacomelli, G. in Cochrane Database of Systematic Reviews (John Wiley \& Sons, Ltd, 1996).

277. Haegerstrom-Portnoy, G., Schneck, M. E., Lott, L. A. \& Brabyn, J. A. The relation between visual acuity and other spatial vision measures. Optom. Vis. Sci. Off. Publ. Am. Acad. Optom. 77, 653-662 (2000).

278. Jin, C. J., Wu, D. Z. \& Wu, L. The contrast sensitivity function in low vision. Yan Ke Xue Bao Eye Sci. Yan Ke Xue Bao Bian Ji Bu 8, 45-48 (1992).

279. Horowitz, A. The Prevalence and Consequences of Vision Impairment in Later Life. LWW (2004). Available at: http://journals.Iww.com/topicsingeriatricrehabilitation/Fulltext/2004/07000/T he_Prevalence_and_Consequences_of_Vision.6.aspx. (Accessed: 24th September 2015) 
280. Kempen, G. I. J. M. \& Zijlstra, G. A. R. Clinically Relevant Symptoms of Anxiety and Depression in Low-Vision Community-Living Older Adults. Am. J. Geriatr. Psychiatry 22, 309-313 (2014).

281. Kempen, G. I. J. M., Ranchor, A. V., Ambergen, T. \& Zijlstra, G. A. R. The mediating role of disability and social support in the association between low vision and depressive symptoms in older adults. Qual. Life Res. 23, 1039-1043 (2014).

282. Horowitz, A., Reinhardt, J. P., Boerner, K. \& Travis, L. A. The influence of health, social support quality and rehabilitation on depression among disabled elders. Aging Ment. Health 7, 342-350 (2003).

283. Boyd, R. \& Stevens, J. A. Falls and fear of falling: burden, beliefs and behaviours. Age Ageing 38, 423-428 (2009).

284. White, U. E., Black, A. A., Wood, J. M. \& Delbaere, K. Fear of falling in vision impairment. Optom. Vis. Sci. Off. Publ. Am. Acad. Optom. 92, 730735 (2015).

285. Ramulu, P. Y. et al. Fear of Falling and Visual Field Loss from Glaucoma. Ophthalmology 119, 1352-1358 (2012).

286. Van Landingham, S. W., Massof, R. W., Chan, E., Friedman, D. S. \& Ramulu, P. Y. Fear of falling in age-related macular degeneration. BMC Ophthalmol. 14, 10 (2014).

287. Merskey, H. \& Bogduk, N. Taxonomy of pain terms \& definitions. (Seattle: IASP Press, 1994).

288. Turk, D. C. \& Okifuji, A. Psychological factors in chronic pain: Evolution and revolution. J. Consult. Clin. Psychol. 70, 678-690 (2002). 
289. Júnior, S., João, J., Nicholas, M. K., Pimenta, C. A. de M. \& Asghari, A. Psychosocial predictors of pain, incapacity and depression in Brazilian chronic pain patients. Rev. Dor 13, 111-118 (2012).

290. Andrasik, F., Flor, H. \& Turk, D. C. An expanded view of psychological aspects in head pain: the biopsychosocial model. Neurol. Sci. 26, s87-s91 (2005).

291. McParland, J. L. \& Knussen, C. Just world beliefs moderate the relationship of pain intensity and disability with psychological distress in chronic pain support group members. Eur. J. Pain 14, 71-76 (2010).

292. Yip, C. H. T., Chiu, T. T. W. \& Poon, A. T. K. The relationship between head posture and severity and disability of patients with neck pain. Man. Ther. 13, 148-154 (2008).

293. Diab, A. A. \& Moustafa, I. M. The efficacy of forward head correction on nerve root function and pain in cervical spondylotic radiculopathy: a randomized trial. Clin. Rehabil. 26, 351-361 (2012).

294. Crowe, L. \& FitzGerald, M. Speed Reading - Harness Your Computer's Power to Triple Your Reading Speed! (Cucoco Ltd., 2005).

295. Wang, Y., Bao, J., Ou, L., Thorn, F. \& Lu, F. Reading behavior of emmetropic schoolchildren in China. Vision Res. 86, 43-51 (2013).

296. Kwon, M., Legge, G. E. \& Dubbels, B. R. Developmental changes in the visual span for reading. Vision Res. 47, 2889-2900 (2007).

297. Ko, P., Mohapatra, A., Bailey, I. L., Sheedy, J. \& Rempel, D. M. Effect of font size and glare on computer tasks in young and older adults. Optom. Vis. Sci. Off. Publ. Am. Acad. Optom. 91, 682-689 (2014). 
298. Bowers, A. R. Eye movements and reading with plus-lens magnifiers. Optom. Vis. Sci. Off. Publ. Am. Acad. Optom. 77, 25-33 (2000).

299. Needle, J. L., Fawcett, A. J. \& Nicolson, R. I. Balance and dyslexia: An investigation of adults' abilities. European Journal of Cognitive Psychology 18, (2006).

300. Chen, E. W., Fu, A. S. N., Chan, K. M. \& Tsang, W. W. N. The effects of Tai Chi on the balance control of elderly persons with visual impairment: a randomised clinical trial. Age Ageing 41, 254-259 (2012).

301. Whittaker, S. \& Lovie-Kitchin, J. Visual Requirements for Reading. : Optometry \& Vision Science. Optometry \& Vision Science (1993). Available at: http://journals.Iww.com/optvissci/Fulltext/1993/01000/Visual_Requirements for_Reading_.10.aspx. (Accessed: 16th November 2015)

302. Burton, R., Crabb, D. P., Smith, N. D., Glen, F. C. \& Garway-Heath, D. F. Glaucoma and reading: exploring the effects of contrast lowering of text. Optom. Vis. Sci. Off. Publ. Am. Acad. Optom. 89, 1282-1287 (2012). 303. Legge, G. E., Rubin, G. S. \& Luebker, A. Psychophysics of reading--V. The role of contrast in normal vision. Vision Res. 27, 1165-1177 (1987).

304. Iliffe, S. et al. Self-reported visual function in healthy older people in Britain: an exploratory study of associations with age, sex, depression, education and income. Fam. Pract. 22, 585-590 (2005).

305. Coco, M. Factores clínicos, psicológicos y sociales predictivos del éxito de un programa de entrenamiento en lectura en pacientes con defectos de campo central. (Universidad de Valladolid, 2011). 
306. Hayman, K. J. et al. Depression in older people: visual impairment and subjective ratings of health. Optom. Vis. Sci. Off. Publ. Am. Acad. Optom. 84, 1024-1030 (2007).

307. Kempen, G. I. J. M., Ballemans, J., Ranchor, A. V., van Rens, G. H. M. B. \& Zijlstra, G. A. R. The impact of low vision on activities of daily living, symptoms of depression, feelings of anxiety and social support in community-living older adults seeking vision rehabilitation services. Qual. Life Res. 21, 1405-1411 (2012).

308. Grant, P., Seiple, W. \& Szlyk, J. P. Effect of depression on actual and perceived effects of reading rehabilitation for people with central vision loss. J. Rehabil. Res. Dev. 48, 1101 (2011).

309. O'Sullivan, P. et al. Evaluation of the flexion relaxation phenomenon of the trunk muscles in sitting. Spine 31, 2009-2016 (2006).

310. Riskind, J. H. They stoop to conquer: Guiding and self-regulatory functions of physical posture after success and failure. J. Pers. Soc. Psychol. 47, 479-493 (1984).

311. Schouwstra, S. J. \& Hoogstraten, J. Head position and spinal position as determinants of perceived emotional state. Percept. Mot. Skills 81, 673674 (1995).

312. Wilson, V. E. \& Peper, E. The effects of upright and slumped postures on the recall of positive and negative thoughts. Appl. Psychophysiol. Biofeedback 29, 189-195 (2004).

313. Balzini, L. et al. Clinical characteristics of flexed posture in elderly women. J. Am. Geriatr. Soc. 51, 1419-1426 (2003). 
314. Wada, T. [Functional decline in older people]. Nihon Rinsho Jpn. J. Clin. Med. 71, 1727-1732 (2013).

315. Soyupek, F. et al. Evaluation of hand functions in women with polycystic ovary syndrome. Gynecol. Endocrinol. Off. J. Int. Soc. Gynecol. Endocrinol. 24, 571-575 (2008).

316. Penta, M., Tesio, L., Arnould, C., Zancan, A. \& Thonnard, J.-L. The ABILHAND Questionnaire as a Measure of Manual Ability in Chronic Stroke Patients Rasch-Based Validation and Relationship to Upper Limb Impairment. Stroke 32, 1627-1634 (2001).

317. Raccio-Robak, N., Mcerlean, M. A., Fabacher, D. A., Milano, P. M. \& Verdile, V. P. Socioeconomic and health status differences between depressed and nondepressed ED elders. Am. J. Emerg. Med. 20, 71-73 (2002).

318. Lenze, E. J. et al. The course of functional decline in older people with persistently elevated depressive symptoms: longitudinal findings from the Cardiovascular Health Study. J. Am. Geriatr. Soc. 53, 569-575 (2005).

319. Noël, P. H. et al. Depression and Comorbid Illness in Elderly Primary Care Patients: Impact on Multiple Domains of Health Status and Well-being. Ann. Fam. Med. 2, 555-562 (2004).

320. Zijlstra, G. a. R. et al. Prevalence and correlates of fear of falling, and associated avoidance of activity in the general population of communityliving older people. Age Ageing 36, 304-309 (2007).

321. Painter, J. A. et al. Fear of Falling and Its Relationship With Anxiety, Depression, and Activity Engagement Among Community-Dwelling Older Adults. Am. J. Occup. Ther. 66, 169-176 (2012). 
322. Austin, N., Devine, A., Dick, I., Prince, R. \& Bruce, D. Fear of Falling in Older Women: A Longitudinal Study of Incidence, Persistence, and Predictors. J. Am. Geriatr. Soc. 55, 1598-1603 (2007).

323. Mane, A. B., Sanjana, T., Patil, P. R. \& Sriniwas, T. Prevalence and correlates of fear of falling among elderly population in urban area of Karnataka, India. J. -Life Health 5, 150-155 (2014).

324. Pinheiro, M. B. et al. Symptoms of depression as a prognostic factor for low back pain: a systematic review. Spine J. Off. J. North Am. Spine Soc. (2015). doi:10.1016/j.spinee.2015.10.037

325. Costa, E. C. V., Vale, S., Sobral, M. \& Graça Pereira, M. Illness perceptions are the main predictors of depression and anxiety symptoms in patients with chronic pain. Psychol. Health Med. 1-13 (2015).

doi:10.1080/13548506.2015.1109673 

ANEXO I 



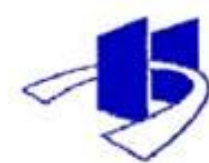

Sacyl

\section{COMITÉ ÉTICO DE INVESTIGACIÓN CLÍNICA ÁREA DE SALUD VALLADOLID - ESTE (CEIC-VA-ESTE-HCUV)}

Valladolid a 22 de Mayo de 2014

En la reunión del CEIC ÁREA DE SALUD VALLADOLID - ESTE del 22 de Mayo de 2014 , se procedió a la evaluación de los aspectos éticos del siguiente proyecto de investigación.

A continuación les señalo los acuerdos tomados por el CEIC ÁREA DE SALUD VALLADOLID - ESTE en relación a dicho Proyecto de Investigación:

\begin{tabular}{|c|c|c|}
\hline PI $14-161$ & $\begin{array}{l}\text { DISENO Y APLICACIÓN DE UN } \\
\text { PROGRAMA FISIOTERAPÉUTICO } \\
\text { PARA PERSONAS MAYORES CON } \\
\text { BAJA VISIÓN }\end{array}$ & $\begin{array}{l}\text { IOBA } \\
\text { IP: BEGOÑA COCO MARTIN, VALLE } \\
\text { FLORES LUCAS } \\
\text { EQUIPO: ANA PATRICIA DE } \\
\text { QUEIROZ BARBOSA } \\
\text { Recibido: } 07-03-2014\end{array}$ \\
\hline
\end{tabular}

Considerando que el Proyecto contempla los Convenios y Normas establecidos en la legislación española en el ámbito de la investigación biomédica, la protección de datos de carácter personal y la bioética, se hace constar el informe favorable y la aceptación del Comité Ético de Investigación Clínica del Área de Salud Valladolid Este para que sea llevado a efecto dicho Proyecto de Investigación.

Un cordial saludo.

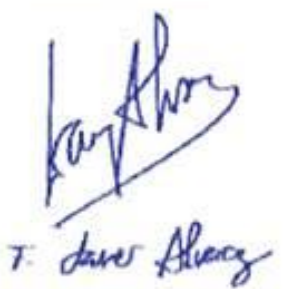

Dr. F. Javier Álvarez,

CEIC Área de Salud Valladolid Este -

Hospital Clinico Universitario de Valladolid

Farmacología

Facultad de Medicina,

Universidad de Valladolid,

c/ Ramón y Cajal 7,47005 Valladolid

alvarez@med.uva.es

jalvarezgo@saludcastillayleon.es

tel: 983423077 

ANEXO II 

INFORMACIÓN SOBRE EL TRABAJO DE INVESTIGACIÓN

"Efectos de la postura corporal, equilibrio y destreza manual en la velocidad lectora en personas mayores con y sin baja visión"

Grupo de investigación: Ana Patrícia de Queiroz Barbosa, Dra. Valle Flores Lucas, Dra. Ma Begoña Coco Martín, Dra. Teresa del Álamo Martín.

Sede donde se realizará el estudio: IOBA

Nombre del paciente:

A usted se le está invitando a participar en este estudio. Antes de decidir si participa o no, debe conocer y comprender cada uno de los siguientes apartados. Este proceso se conoce como consentimiento informado. Siéntase con absoluta libertad para preguntar sobre cualquier aspecto que le ayude a aclarar sus dudas al respecto. Una vez que haya comprendido el estudio y si usted desea participar, entonces se le pedirá que firme esta forma de consentimiento, de la cual se le entregará una copia firmada y fechada.

\section{PARTICIPACIÓN VOLUNTARIA}

Debe saber que su participación en este estudio es voluntaria y que puede decidir no participar o cambiar su decisión y retirar su consentimiento en cualquier momento sin que por ello se altere su relación con los médicos/investigadores de la institución encargada de la realización del estudio.

\section{DESCRIPCIÓN GENERAL DEL ESTUDIO}

Serán evaluados los efectos físicos (alteraciones del movimiento, funcionalidad y postura, así como el dolor) que se deriven de la baja visión sobre las personas mayores que sufren dicha condición, mediante pruebas físicas específicas. Además de eso, verificaremos la relación entre la velocidad lectora con ayudas ópticas y la destreza manual.

El estudio consta de 2 visitas:

Visita 1: Explicar en qué consiste el estudio, sus riesgos y beneficios para la firma del Consentimiento Informado. Tras la aceptación en participar y la firma del $\mathrm{Cl}$, se aplicarán algunas de las pruebas de evaluación.

Visita 2: Aplicar las últimas pruebas de evaluación que no han sido aplicadas en la visita anterior. 


\section{CONSECUENCIAS, RIESGOS Y BENEFICIOS}

Todas las pruebas que le realizaremos No son invasivas. En cualquier caso, hay que informar de algunos efectos que se pueden producir durante el desarrollo de la evaluación. Hay una prueba de equilibrio y una de evaluación postural que pueden producir algún riesgo de caídas, aunque estos son muy improbables pues son pruebas sencillas y movimientos cotidianos.

- Evaluación postural: Los participantes serán fotografiados en la posición de pies, en los ángulos anterior, posterior, lateral izquierda y derecha. Habrá dos cámaras fotográficas, una delante y la otra al lado del participante, así que tomaremos las fotos en posición anterior y lateral derecha al mismo tiempo. Después el participante cambia de posición haciendo un giro de $180^{\circ}$ para que se tomen las fotos en vista posterior y lateral izquierda. Durante la foto, el participante debe estar en ropa interior.

- Timed Up and Go Test (TUG): Es una prueba de equilibrio dinámico, donde será medido el tiempo en segundos para una persona que está sentada, en una silla, tarda en levantarse, caminar 3 metros, girar, caminar de regreso a la silla, y sentarse. La persona debe llevar calzado regular y la ayuda habitual para caminar. El asiento de la silla debe tener una altura de aproximadamente 46 centímetros y el brazo de la silla debe tener 65 centímetros de altura.

Con su participación en el estudio se podrá conocer las repercusiones de la baja visión en las alteraciones del movimiento, funcionalidad y postura de las personas mayores, ayudando por tanto en la prevención de sus efectos en los diversos aspectos de la vida además de posibilitar y en el caso de padecer dicha enfermedad se beneficiará de los hallazgos obtenidos tras finalizar el estudio.

\section{CONFIDENCIALIDAD}

El acceso a sus datos clínicos y asistenciales se realizará guardando la más estricta confidencialidad de forma que no se viola la intimidad personal de los sujetos participantes en el estudio. Sus datos serán objeto de un tratamiento disociado, de modo que la información que se obtenga no pueda asociarse a una persona identificada o identificable. Durante la realización del estudio se garantizará el estricto cumplimiento de la Ley 15/1999 de 13 de diciembre de Protección de datos Personales. El investigador, cuando procese y trate sus datos, tomará las medidas oportunas para protegerlos y evitar el acceso a los mismos de terceros no autorizados. Ud. puede revocar el consentimiento para el tratamiento de sus datos personales dirigiéndose a cualquier investigador partícipe del estudio. Su nombre o identidad no aparecerán en ningún informe, resultado o publicación relacionada con el estudio.

Según la Ley 15/1999 de 13 de Diciembre, el consentimiento para el tratamiento de sus datos personales y para su cesión es revocable. Ud. puede ejercer el derecho de acceso, rectificación y cancelación dirigiéndose al investigador, el cual lo pondrá en conocimiento del promotor. 


\title{
DECLARACIÓN DE CONSENTIMIENTO INFORMADO
}

\section{Título del Estudio:}

\section{"Efectos de la postura corporal, equilibrio y destreza manual en la velocidad lectora en personas mayores con y sin baja visión"}

\begin{abstract}
Al firmar abajo, yo declaro que:
He leído, o me han leído, y entiendo completamente el contenido del formulario de información adjunto.

He tenido la oportunidad de preguntar y obtener respuestas satisfactorias a cada una de mis preguntas.
\end{abstract}

Acepto de forma voluntaria participar en este estudio de investigación y sé que puedo retirarme del estudio en cualquier momento.

La profesional del Grupo de Investigación de la Universidad de Valladolid: Ana Patrícia de Queiroz Barbosa, Dirección: Instituto Universitario de Oftalmología Aplicada, Valladolid; Número de Teléfono: 983423 559; me ha explicado la información para el paciente, el formulario de consentimiento y comprendo lo que implica la investigación.

He comprendido completamente que los representantes del patrocinador, el Comité Ético Independiente o los representantes de las autoridades regulatorias pueden examinar mis registros médicos donde aparece mi nombre para verificar la exactitud de la información obtenida y entiendo que estas personas tendrán el deber de manejar esta información con confidencialidad utilizándola solamente con un objetivo legítimo para la salud pública.

Se me entregará una copia firmada y fechada de este formulario de consentimiento para mis propios archivos.

Nombre del Paciente

Firma

Fecha

(Debe ser firmado y fechado por el paciente)

Nombre del profesional que participó en la discusión del CI Ana Patrícia de Queiroz Barbosa

Firma

Fecha

(Debe ser firmado y fechado por la persona que explicó el consentimiento informado) 




\section{PROTOCOLO DE EVALUACIÓN}

I- Datos de identificación

Participante:

Fecha:

Nombre:

Fecha de nacimiento:

Edad:

Profesión:

Estado civil:

Dirección:

Teléfono:

Altura:

Peso:

IMC:

Miembro

dominante:

¿Utiliza usted alguna medicación?

¿Ha tenido caídas en los últimos tres años?

II- Datos Clínicos Actuales

¿Siente usted dolor en alguna parte del cuerpo? ¿Dónde? ¿Hace cuánto?

( ) Cabeza

( ) Columna cervical

( ) Columna torácica

( ) Columna lumbar

( ) Pelvis

( ) Cadera

( ) Muslo

( ) Rodilla

( ) Pierna

( ) Tobillo

( ) Pie

( ) Hombro

( ) Brazo

( ) Codo

( ) Antebrazo

( ) Muñeca

( ) Mano

( ) Dedos 
Anexo III

Compruebe en la línea la zona que corresponda a su nivel de dolor.

Sin dolor Máximo dolor

Sin dolor Máximo dolor

Sin dolor Máximo dolor

Sin dolor Máximo dolor 


\section{MINI MENTAL STATE EXAMINATION (MMSE)}

Basado en Folstein et al. (1975), Lobo et al. (1979)

\begin{tabular}{|c|c|}
\hline $\begin{array}{lr}\text { Nombre: } & \\
\text { Fecha: } & \text { F. nacimiento: } \\
\text { Estudios/Profesión: } & \text { N. } H^{\underline{a}} \text { : } \\
\text { Observaciones: } & \\
\end{array}$ & $\begin{array}{l}\text { Varón [ ] } \\
\text { Edad: }\end{array}$ \\
\hline $\begin{array}{l}\text { ¿En qué año estamos? 0-1 } \\
\text { ¿En qué estación? 0-1 } \\
\text { ¿En qué día (fecha)? } 0-1 \\
\text { ¿En qué mes? 0-1 } \\
\text { ¿En qué día de la semana? 0-1 }\end{array}$ & $\begin{array}{c}\text { ORIENTACIÓN } \\
\text { TEMPORAL (Máx.5) }\end{array}$ \\
\hline $\begin{array}{l}\text { En qué hospital (o lugar) estamos? } 0-1 \\
\text { En qué piso (o planta, sala, servicio)? } 0-1 \\
\text { En qué pueblo (ciudad)? } 0-1 \\
\text { En qué provincia estamos? } 0-1 \\
\text { En qué país (o nación, autonomía)? } 0-1\end{array}$ & $\begin{array}{l}\text { ORIENTACIÓN } \\
\text { ESPACIAL (Máx.5) }\end{array}$ \\
\hline $\begin{array}{l}\text { Nombre tres palabras Peseta-Caballo-Manzana (o Balón- } \\
\text { Bandera-Árbol) a razón de } 1 \text { por segundo. Luego se pide al } \\
\text { paciente que las repita. Esta primera repetición otorga la } \\
\text { puntuación. Otorgue } 1 \text { punto por cada palabra correcta, pero } \\
\text { continúe diciéndolas hasta que el sujeto repita las } 3 \text {, hasta } \\
\text { un máximo de } 6 \text { veces. } \\
\text { Peseta } 0-1 \text { Caballo } 0-1 \text { Manzana } 0-1 \\
\text { (Balón } 0-1 \text { Bandera } 0-1 \text { Árbol } 0-1 \text { ) }\end{array}$ & $\begin{array}{l}\text { FIJACIÓN-Recuerdo } \\
\text { Inmediato (Máx.3) }\end{array}$ \\
\hline 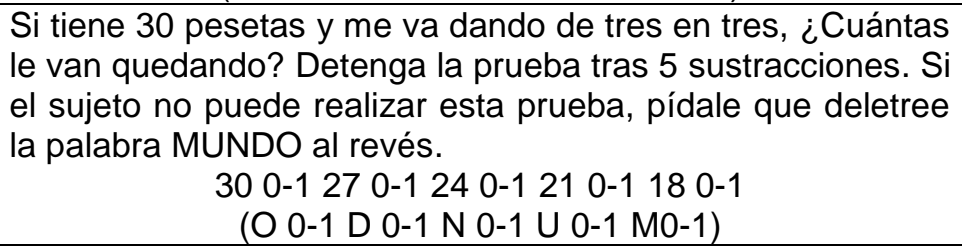 & $\begin{array}{l}\text { ATENCIÓN- } \\
\text { CÁLCULO } \\
\text { (Máx.5) }\end{array}$ \\
\hline $\begin{array}{l}\text { Preguntar por las tres palabras mencionadas anteriormente. } \\
\text { Peseta 0-1 Caballo 0-1 Manzana 0-1 } \\
\text { (Balón 0-1 Bandera 0-1 Árbol 0-1) }\end{array}$ & $\begin{array}{l}\text { RECUERDO diferido } \\
\text { (Máx.3) }\end{array}$ \\
\hline $\begin{array}{l}\text {.DENOMINACIÓN. Mostrarle un lápiz o un bolígrafo y } \\
\text { preguntar ¿qué es esto?. Hacer lo mismo con un reloj de } \\
\text { pulsera. Lápiz 0-1 Reloj 0-1 } \\
\text {.REPETICIÓN. Pedirle que repita la frase: "ni sí, ni no, ni } \\
\text { pero" (o "En un trigal había } 5 \text { perros") 0-1 } \\
\text {.ÓRDENES. Pedirle que siga la orden: "coja un papel con la } \\
\text { mano derecha, dóblelo por la mitad, y póngalo en el suelo". } \\
\text { Coge con mano d. 0-1 dobla por mitad 0-1 pone en suelo 0-1 } \\
\text {.LECTURA. Escriba legiblemente en un papel "Cierre los } \\
\text { ojos". Pídale que lo lea y haga lo que dice la frase 0-1 } \\
\text {.ESCRITURA. Que escriba una frase (con sujeto y } \\
\text { predicado) 0-1 } \\
\text {.COPIA. Dibuje } 2 \text { pentágonos intersectados y pida al sujeto } \\
\text { que los copie tal cual. Para otorgar un punto deben estar } \\
\text { presentes los } 10 \text { ángulos y la intersección. 0-1 } \\
\text { Puntuaciones de referencia 27 ó más: normal } 24 \text { ó } \\
\text { menos: sospecha patológica 12-24: deterioro } \mathbf{9 - 1 2} \text { : } \\
\text { demencia }\end{array}$ & $\begin{array}{l}\text { LENGUAJE (Máx.9) } \\
\text { Puntuación Total } \\
\text { (Máx.: } 30 \text { puntos) }\end{array}$ \\
\hline
\end{tabular}




\section{ESCALA DE DEPRESIÓN GERIÁTRICA (GDS)}

Escoja la respuesta adecuada según cómo se sintió usted la semana pasada.

1. ¿Está usted básicamente, satisfecho(a) con su vida? SI / NO

2. ¿Ha suspendido usted muchas de sus actividades e intereses? SI / NO

3. ¿Siente usted que su vida está vacía? SI / NO

4. ¿Se aburre usted a menudo? SI / NO

5. ¿Está usted de buen humor la mayor parte del tiempo? SI / NO

6. ¿Tiene usted miedo de que algo malo le vaya a pasar? SI / NO

7. ¿Se siente feliz la mayor parte del tiempo? SI / NO

8. ¿Se siente usted a menudo indefenso(a)? SI / NO

9. ¿Prefiere usted quedarse en la casa, en vez de salir y hacer cosas nuevas?

SI / NO

10. ¿Con respecto a su memoria: ¿Siente usted que tiene más problemas que la mayoría de la gente? SI / NO

11. ¿Piensa usted que es maravilloso estar vivo(a) en este momento? SI / NO

12. ¿De la forma de cómo se siente usted en este momento, ¿Se siente usted inútil? SI / NO

13. ¿Se siente usted con mucha energía? SI / NO

14. ¿Siente usted que su situación es irremediable? SI / NO

15. ¿Piensa usted que la mayoría de las personas están en mejores condiciones que usted? SI / NO

Las respuestas en negrita indican depresión. Asigne 1 punto por cada respuesta en negrita.

Un puntaje $>5$ puntos parece indicar depresión.

Un puntaje $\geq 10$ puntos es casi siempre un indicador de depresión.

Un puntaje $>5$ puntos debería garantizar la realización de una evaluación integral de seguimiento. 


\section{FES-I}

Ahora le queremos hacer algunas preguntas relacionadas con su preocupación sobre la posibilidad de caerse. Para cada una de las actividades siguientes, por favor haga un círculo en la frase que más se aproxime a su opinión que muestre la medida en que está preocupado/a que pueda caerse si hiciera esta actividad. Por favor conteste pensando en la manera habitual que tiene de realizar la actividad. Si Ud. no realiza actualmente actividad (ej., si alguien compra por usted), por favor conteste en relación a mostrar si usted estaría preocupado/a de caerse SI usted realizara dicha actividad.

$\begin{array}{cccc}\text { En absoluto } & \text { Algo } & \text { Bastante } & \text { Muy } \\ \text { preocupado/a } & \text { preocupado/ } & \text { preocupado/ } & \text { preocupado/a } \\ 1 & \text { a } & \text { a } & 4 \\ & 2 & 3 & \end{array}$

\begin{tabular}{|c|c|c|c|c|c|}
\hline 1 & $\begin{array}{c}\text { Limpiar la casa (ej., barrer, } \\
\text { pasar la aspiradora o } \\
\text { limpiar el polvo) }\end{array}$ & $\square$ & $\square$ & $\square$ & $\square$ \\
\hline 2 & Vestirse o desvestirse & $\square$ & $\square$ & $\square$ & $\square$ \\
\hline 3 & Preparar comidas cada día & $\square$ & $\square$ & $\square$ & $\square$ \\
\hline 4 & Bañarse o ducharse & $\square$ & $\square$ & $\square$ & $\square$ \\
\hline 5 & Ir a la compra & $\square$ & $\square$ & $\square$ & $\square$ \\
\hline 6 & $\begin{array}{c}\text { Sentarse o levantarse de } \\
\text { una silla }\end{array}$ & $\square$ & $\square$ & $\square$ & $\square$ \\
\hline 7 & Subir o bajar escaleras & $\square$ & $\square$ & $\square$ & $\square$ \\
\hline 8 & $\begin{array}{l}\text { Caminar por el barrio (o } \\
\text { vecindad, fuera de casa) }\end{array}$ & $\square$ & $\square$ & $\square$ & $\square$ \\
\hline 9 & $\begin{array}{l}\text { Coger algo alto (por encima } \\
\text { de su cabeza) o en el suelo }\end{array}$ & $\square$ & $\square$ & $\square$ & $\square$ \\
\hline 10 & $\begin{array}{l}\text { Ir a contestar el teléfono } \\
\text { antes de que deje de sonar }\end{array}$ & $\square$ & $\square$ & $\square$ & $\square$ \\
\hline 11 & $\begin{array}{c}\text { Caminar sobre una } \\
\text { superficie resbaladiza (ej., } \\
\text { mojada o con hielo) }\end{array}$ & $\square$ & $\square$ & $\square$ & $\square$ \\
\hline 12 & Visitar a un amigo o familiar & $\square$ & $\square$ & $\square$ & $\square$ \\
\hline 13 & $\begin{array}{l}\text { Caminar en un lugar con } \\
\text { mucha gente }\end{array}$ & $\square$ & $\square$ & $\square$ & $\square$ \\
\hline 14 & $\begin{array}{l}\text { Caminar en una superficie } \\
\text { irregular (ej., pavimento en } \\
\text { mal estado, sin asfaltar) }\end{array}$ & $\square$ & $\square$ & $\square$ & $\square$ \\
\hline 15 & Subir y bajar una rampa & $\square$ & $\square$ & $\square$ & $\square$ \\
\hline 16 & $\begin{array}{l}\text { Salir a un evento social (por } \\
\text { ejemplo, religioso, reunión } \\
\text { familiar o reunión social) }\end{array}$ & $\square$ & $\square$ & $\square$ & $\square$ \\
\hline
\end{tabular}

FES-I Spanish translated from English by Dr Ramona Lucas 
Anexo III

\section{IReST}

En un pueblecito, un verdulero abrió una tienda justo encima

de un profundo sótano. Cada noche, los ratones

salían a manadas del sótano y entraban en la tienda. Se

comían las manzanas y las peras, las uvas y las nueces, y no se

quedaban cortos con la verdura y las patatas. No había producto

que estuviese a salvo de los pequeños y molestos

roedores entre la medianoche y el amanecer. Mientras

se oyesen ruidos de la calle por el paso

de los coches, se quedaban tranquilos

en el sótano. Pero tan pronto como el reloj del ayuntamiento

daba las doce y se hacía el silencio afuera,

salían a borbotones, se ponían las botas con las frutas y

celebraban banquetes magníficos, cuyos restos

desesperaban al vendedor cada mañana al llegar a la tienda.

Así que decidió defenderse de los ratones. Primero,

puso trampas por todo el local.

$N^{\circ}$ de texto: 1

Nombre del texto: Ratones

$N^{\circ}$ de palabras: 146

$\mathrm{N}^{\circ}$ de carácteres: 813

Velocidad de lectura:

Palabras/minuto (media $\pm \mathrm{DE}$ )

Carácteres/minuto (med 

